\title{
PERIODICITIES OF T-SYSTEMS AND Y-SYSTEMS
}

\author{
REI INOUE, OSAMU IYAMA, ATSUO KUNIBA, TOMOKI \\ NAKANISHI, AND JUNJI SUZUKI
}

\begin{abstract}
The unrestricted T-system is a family of relations in the Grothendieck ring of the category of the finite-dimensional modules of Yangian or quantum affine algebra associated with a complex simple Lie algebra. The unrestricted T-system admits a reduction called the restricted T-system. In this paper we formulate the periodicity conjecture for the restricted T-systems, which is the counterpart of the known and partially proved periodicity conjecture for the restricted Y-systems. Then, we partially prove the conjecture by various methods: the cluster algebra and cluster category method for the simply laced case, the determinant method for types $A$ and $C$, and the direct method for types $A, D$, and $B$ (level 2).
\end{abstract}

\section{$\S 1$. Introduction}

The $Y$-system was introduced as a system of functional relations concerning the solutions of the thermodynamic Bethe ansatz equations for the factorizable scattering theory and the solvable lattice models $[\mathrm{Z}],[\mathrm{KP}],[\mathrm{KN}]$, $[R T V]$. It was conjectured that the solutions of the Y-system have periodicity [Z], [RTV], [KNS1]. Fomin and Zelevinsky proved it for a special case (level 2 case in our terminology) [FZ3] by the cluster algebra approach [FZ1], [FZ2], [FZ4]. Since then, a remarkable link has been established between cluster algebras and cluster categories of the quiver representations (see [BMRRT], [BMR], [CC], [CK1], [CK2], [Kel2], and references therein). Based on this categorification method, Keller recently proved the periodicity of the Y-system for a more general case [Kel2], [Kel3].

Meanwhile, it has been known that the Y-system is related to other systems of relations called the T-system and the Q-system [KP], [KNS1]. The T-system is a family of relations in the Grothendieck ring of the category of the finite-dimensional modules of the Yangian $Y(\mathfrak{g})$ or the quantum affine algebra $U_{q}(\hat{\mathfrak{g}})$ associated with a complex simple Lie algebra $\mathfrak{g}$ [KNS1], [N3], [Her1], [Her2]. As a discrete dynamical system, the T-system can be also

Received December 26, 2003. Revised April 24, 2009. Accepted July 28, 2009. 
viewed as a discrete analogue of the Toda field equation [KOS], [KLWZ]. The Q-system is a degenerated version of the T-system and plays an important role in the algebraic Bethe ansatz method [Ki], [KR], [HKOTY], [KNT]. As a side remark, it may be worth mentioning at this point that " $\mathrm{T}$ " stands for transfer matrix and "Q" stands for quantum character $[\mathrm{Ki} 2]$ in the original literature.

As a more recent development, a connection between the Q-systems and cluster algebras is clarified by $[\mathrm{Ked}],[\mathrm{DiK}]$. Also, a connection between the T-systems (or $q$-characters) and cluster algebras is studied while seeking a natural categorification of cluster algebras by abelian monoidal categories [HL].

Having these results as a background, we make three simple observations:

(1) There are actually two classes of the Y-systems (resp. T-systems), namely, the unrestricted and restricted Y-systems (resp. T-systems). The latter is obtained by a certain reduction from the former. The periodicity property above mentioned is for the restricted Y-systems.

(2) The cluster algebra structure is simpler in the T-systems than in the Y-systems.

(3) The representation theory of quantum affine algebras is more directly connected with the T-systems than with the Y-systems.

These observations motivate us to ask if there is a similar periodicity property for the restricted T-systems, and indeed, there is.

In this paper, we formulate the periodicity conjecture for the restricted T-systems, which is the counterpart of the known and partially proved periodicity conjecture for the restricted Y-systems. Then, we partially prove the conjecture by various methods. We remark that the restricted T-systems are relations in certain quotients of the Grothendieck ring $\operatorname{Rep} U_{q}(\hat{\mathfrak{g}})$, while the T-systems studied in [HL] are relations in certain subrings of $\operatorname{Rep} U_{q}(\hat{\mathfrak{g}})$. Accordingly, the correspondence between the T-systems for the simply laced case and cluster algebras considered here and the one in [HL] are close but slightly different. We also note that the correspondence between the unrestricted T-systems for the simply laced case and cluster algebras is described in [DiK, Appendix B].

Let us explain the outline of the paper, whose contents are roughly divided into three parts.

In the first part (Section 2) we introduce the unrestricted T-systems together with their associated rings, which we call the unrestricted T-algebras. 
Then, we establish an isomorphism between a subring of the unrestricted Talgebra and the Grothendieck ring of the category of the finite-dimensional representations of an untwisted quantum affine algebra (Corollary 2.9). The relation between the unrestricted $\mathrm{T}$ - and Y-algebras is also given (Theorem 2.12). They provide the representation theoretical background of the periodicity problem we are going to discuss.

In the second part (Sections 3-7) we introduce the level $\ell$ restricted $T$-systems together with their associated rings, which we call the level $\ell$ restricted $T$-algebras, where $\ell$ is an integer greater than or equal to 2 . Then, we formulate the periodicity conjecture (Conjecture 3.11 ) of the restricted T-systems in terms of the restricted T-algebras. This is the main claim of the paper. Conjecture 3.11 is completely parallel to that of the restricted Y-systems (Conjecture 3.12). A detailed summary of our methods and results concerning Conjecture 3.11 is given in Section 3.4. In brief, we study and partially prove the periodicity conjecture by three independent methods: the cluster algebra/category method for the simply laced case in Section 4, the determinant method for types $A$ and $C$ in Sections 5 and 6 , and the direct method for types $A, D$, and $B$ (level 2) in Section 7. In particular, for the simply laced case, the relation between the restricted T-algebras and cluster algebras is clarified in Section 4. For the cluster category method, we follow the ideas of Keller [Kel2] based on Amiot's generalized cluster categories $[\mathrm{A}]$.

In the third part (Sections 8 and 9) we apply the extensions of the above periodicity property to two classes of $\mathrm{T}$ - and $\mathrm{Y}$-systems. In Section 8 we formulate and prove the periodicity property for the restricted $\mathrm{T}$ - and Y-systems at levels 1 and 0 . In Section 9 we formulate the periodicity property also for the restricted T- and Y-systems associated with the twisted quantum affine algebras. It turns out that their periodicity property reduces to that of the untwisted case. We remark that the nonsimply laced Y-systems studied in [FZ2], [Kel2] are identified with certain reductions of the restricted Y-systems belonging to this class (Remark 9.22).

We conclude the paper with a brief remark (Section 10) on a formal correspondence between the periodicity of the T-systems and the $q$-character of the quantum affine algebras at roots of unity. This suggests that there is some further connection between the representation theories of quivers and the quantum affine algebras at roots of unity behind this periodicity phenomenon, possibly through the works of [N1], [N2]. The relation between the 
restricted $\mathrm{T}$ - and $\mathrm{Y}$-algebras and cluster algebras for the nonsimply laced case will be discussed in a separate publication.

Acknowledgments. We thank Sergey Fomin, David Hernandez, Bernhard Keller, Anatol Kirillov, Bernard Leclerc, Hyohe Miyachi, Roberto Tateo, and Andrei Zelevinsky for discussions and communications.

\section{§2. Unrestricted T- and Y-systems}

In this section we introduce the unrestricted $T$ - and $Y$-systems of [KNS1] as a background of the periodicity problem. We also introduce the associated algebras, which we call the unrestricted $T$ - and $Y$-algebras. They are closely connected to the Grothendieck ring of the category of the finitedimensional $Y(\mathfrak{g})$-modules or $U_{q}(\hat{\mathfrak{g}})$-modules. The content of this section is rather independent of the rest of the paper.

\subsection{Unrestricted T-systems}

Throughout the paper, a "ring" means a commutative ring (algebra over $\mathbb{Z}$ ) with identity element. For a ring $R, R^{\times}$denotes the set of all the invertible elements of $R$. The set of all the positive integers is denoted by $\mathbb{N}$.

Let $X_{r}$ be a Dynkin diagram of finite type with rank $r$, and let $I=$ $\{1, \ldots, r\}$ be the enumeration of the vertices of $X_{r}$ as in Figure 1 . We follow [Ka] except for $E_{6}$, for which we choose the one naturally corresponding to the enumeration of the twisted affine diagram $E_{6}^{(2)}$ in Section 9.

Let $C=\left(C_{a b}\right), C_{a b}=2\left(\alpha_{a}, \alpha_{b}\right) /\left(\alpha_{a}, \alpha_{a}\right)$, be the Cartan matrix of $X_{r}$. We set numbers $t$ and $t_{a}(a \in I)$ by

$$
\begin{aligned}
t & = \begin{cases}1 & X_{r}: \text { simply laced, } \\
2 & X_{r}=B_{r}, C_{r}, F_{4}, \\
3 & X_{r}=G_{2},\end{cases} \\
t_{a} & = \begin{cases}1 & X_{r}: \text { simply laced, } \\
1 & X_{r}: \text { nonsimply laced, } \alpha_{a}: \text { long root } \\
t & X_{r}: \text { nonsimply laced, } \alpha_{a}: \text { short root. }\end{cases}
\end{aligned}
$$

Let $U$ be either the complex plane $\mathbb{C}$ or the cylinder $\mathbb{C}_{\xi}:=\mathbb{C} /(2 \pi \sqrt{-1} / \xi) \mathbb{Z}$ for some $\xi \in \mathbb{C} \backslash 2 \pi \sqrt{-1} \mathbb{Q}$.

Definition 2.1. The unrestricted T-system $\mathbb{T}\left(X_{r}\right)$ of type $X_{r}$ is the following system of relations for a family of variables $T=\left\{T_{m}^{(a)}(u) \mid a \in I, m \in\right.$ 
$A_{r}$

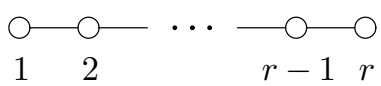

$C_{r}$

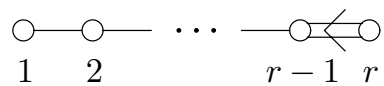

$E_{6}$

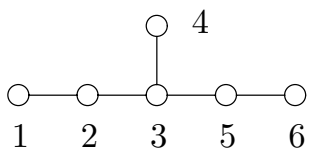

$E_{8}$

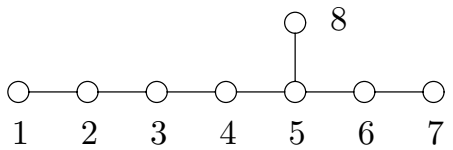

$F_{4}$

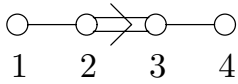

$B_{r}$

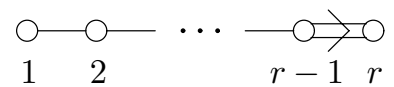

$D_{r}$

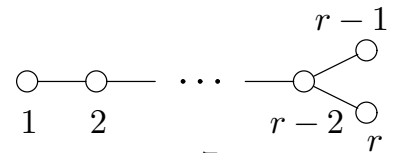

$E_{7}$

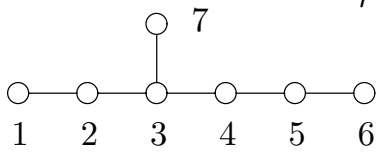

Figure 1: The Dynkin diagrams $X_{r}$ and their enumerations

$\mathbb{N}, u \in U\}$, where $T_{m}^{(0)}(u)=T_{0}^{(a)}(u)=1$ if they occur in the right-hand sides in the relations.

(Here and throughout the paper, $2 m$ (resp. $2 m+1$ ) in the left-hand side, e.g., represents elements $2,4, \ldots$ (resp. $1,3, \ldots)$ ).

For simply laced $X_{r}$,

$$
T_{m}^{(a)}(u-1) T_{m}^{(a)}(u+1)=T_{m-1}^{(a)}(u) T_{m+1}^{(a)}(u)+\prod_{b \in I: C_{a b}=-1} T_{m}^{(b)}(u) .
$$

For $X_{r}=B_{r}$,

$$
\begin{aligned}
T_{m}^{(a)}(u-1) T_{m}^{(a)}(u+1)= & T_{m-1}^{(a)}(u) T_{m+1}^{(a)}(u) \\
& +T_{m}^{(a-1)}(u) T_{m}^{(a+1)}(u) \quad(1 \leq a \leq r-2), \\
T_{m}^{(r-1)}(u-1) T_{m}^{(r-1)}(u+1)= & T_{m-1}^{(r-1)}(u) T_{m+1}^{(r-1)}(u)+T_{m}^{(r-2)}(u) T_{2 m}^{(r)}(u), \\
T_{2 m}^{(r)}\left(u-\frac{1}{2}\right) T_{2 m}^{(r)}\left(u+\frac{1}{2}\right)= & T_{2 m-1}^{(r)}(u) T_{2 m+1}^{(r)}(u) \\
& +T_{m}^{(r-1)}\left(u-\frac{1}{2}\right) T_{m}^{(r-1)}\left(u+\frac{1}{2}\right), \\
T_{2 m+1}^{(r)}\left(u-\frac{1}{2}\right) T_{2 m+1}^{(r)}\left(u+\frac{1}{2}\right)= & T_{2 m}^{(r)}(u) T_{2 m+2}^{(r)}(u)+T_{m}^{(r-1)}(u) T_{m+1}^{(r-1)}(u) .
\end{aligned}
$$


For $X_{r}=C_{r}$,

$$
\begin{aligned}
(2.4) T_{m}^{(a)}\left(u-\frac{1}{2}\right) T_{m}^{(a)}\left(u+\frac{1}{2}\right)= & T_{m-1}^{(a)}(u) T_{m+1}^{(a)}(u) \\
& +T_{m}^{(a-1)}(u) T_{m}^{(a+1)}(u) \quad(1 \leq a \leq r-2), \\
T_{2 m}^{(r-1)}\left(u-\frac{1}{2}\right) T_{2 m}^{(r-1)}\left(u+\frac{1}{2}\right)= & T_{2 m-1}^{(r-1)}(u) T_{2 m+1}^{(r-1)}(u) \\
& +T_{2 m}^{(r-2)}(u) T_{m}^{(r)}\left(u-\frac{1}{2}\right) T_{m}^{(r)}\left(u+\frac{1}{2}\right) \\
T_{2 m+1}^{(r-1)}\left(u-\frac{1}{2}\right) T_{2 m+1}^{(r-1)}\left(u+\frac{1}{2}\right)= & T_{2 m}^{(r-1)}(u) T_{2 m+2}^{(r-1)}(u) \\
& +T_{2 m+1}^{(r-2)}(u) T_{m}^{(r)}(u) T_{m+1}^{(r)}(u) \\
T_{m}^{(r)}(u-1) T_{m}^{(r)}(u+1)= & T_{m-1}^{(r)}(u) T_{m+1}^{(r)}(u)+T_{2 m}^{(r-1)}(u) .
\end{aligned}
$$

For $X_{r}=F_{4}$,

$$
\begin{aligned}
T_{m}^{(1)}(u-1) T_{m}^{(1)}(u+1)= & T_{m-1}^{(1)}(u) T_{m+1}^{(1)}(u)+T_{m}^{(2)}(u), \\
T_{m}^{(2)}(u-1) T_{m}^{(2)}(u+1)= & T_{m-1}^{(2)}(u) T_{m+1}^{(2)}(u)+T_{m}^{(1)}(u) T_{2 m}^{(3)}(u), \\
T_{2 m}^{(3)}\left(u-\frac{1}{2}\right) T_{2 m}^{(3)}\left(u+\frac{1}{2}\right)= & T_{2 m-1}^{(3)}(u) T_{2 m+1}^{(3)}(u) \\
& +T_{m}^{(2)}\left(u-\frac{1}{2}\right) T_{m}^{(2)}\left(u+\frac{1}{2}\right) T_{2 m}^{(4)}(u), \\
T_{2 m+1}^{(3)}\left(u-\frac{1}{2}\right) T_{2 m+1}^{(3)}\left(u+\frac{1}{2}\right)= & T_{2 m}^{(3)}(u) T_{2 m+2}^{(3)}(u) \\
& +T_{m}^{(2)}(u) T_{m+1}^{(2)}(u) T_{2 m+1}^{(4)}(u), \\
T_{m}^{(4)}\left(u-\frac{1}{2}\right) T_{m}^{(4)}\left(u+\frac{1}{2}\right)= & T_{m-1}^{(4)}(u) T_{m+1}^{(4)}(u)+T_{m}^{(3)}(u) .
\end{aligned}
$$

For $X_{r}=G_{2}$,

$$
\begin{aligned}
T_{3 m}^{(2)}\left(u-\frac{1}{3}\right) T_{3 m}^{(2)}\left(u+\frac{1}{3}\right)= & T_{3 m-1}^{(2)}(u) T_{3 m+1}^{(2)}(u) \\
& +T_{m}^{(1)}\left(u-\frac{2}{3}\right) T_{m}^{(1)}(u) T_{m}^{(1)}\left(u+\frac{2}{3}\right), \\
T_{3 m+1}^{(2)}\left(u-\frac{1}{3}\right) T_{3 m+1}^{(2)}\left(u+\frac{1}{3}\right)= & T_{3 m}^{(2)}(u) T_{3 m+2}^{(2)}(u)
\end{aligned}
$$




$$
\begin{aligned}
& +T_{m}^{(1)}\left(u-\frac{1}{3}\right) T_{m}^{(1)}\left(u+\frac{1}{3}\right) T_{m+1}^{(1)}(u), \\
T_{3 m+2}^{(2)}\left(u-\frac{1}{3}\right) T_{3 m+2}^{(2)}\left(u+\frac{1}{3}\right)= & T_{3 m+1}^{(2)}(u) T_{3 m+3}^{(2)}(u) \\
& +T_{m}^{(1)}(u) T_{m+1}^{(1)}\left(u-\frac{1}{3}\right) T_{m+1}^{(1)}\left(u+\frac{1}{3}\right) .
\end{aligned}
$$

The choice of the domain $U=\mathbb{C}_{\xi}$ of the parameter $u$ effectively imposes an additional periodic condition:

$$
T_{m}^{(a)}(u)=T_{m}^{(a)}\left(u+\frac{2 \pi \sqrt{-1}}{\xi}\right) .
$$

By the assumption, we have $2 \pi \sqrt{-1} / \xi \notin \mathbb{Q}$ so that it is compatible with the relations $\mathbb{T}\left(X_{r}\right)$.

REMARK 2.2. Originally, the system $\mathbb{T}\left(X_{r}\right)$ was introduced in [KNS1] as a family of relations in the ring of commuting transfer matrices of the solvable lattice models. For example, for $X_{r}=A_{r}$, the relations in (2.2) are the Jacobi identities among the Jacobi-Trudi-type determinantal expression of the transfer matrices in $[\mathrm{BR}]$. The T-system is a natural affinization of the $Q$-system of $[\mathrm{Ki}],[\mathrm{KR}]$ (see Appendix A.1), and the idea behind the both systems was the existence of a conjectured family of exact sequences among the Kirillov-Reshetikhin modules [KR], [CP1], [KNS1] of the Yangian $Y(\mathfrak{g})$ and/or the untwisted quantum affine algebra $U_{q}(\hat{\mathfrak{g}})$ associated with the complex simple Lie algebra $\mathfrak{g}$ of type $X_{r}[\mathrm{D} 1],[\mathrm{D} 2],[\mathrm{J}]$. The choice $U=\mathbb{C}$ corresponds to the $Y(\mathfrak{g})$ case, while the choice $U=\mathbb{C}_{\xi}$ corresponds to the $U_{q}(\hat{\mathfrak{g}})$ case, as explained below. For $U_{q}\left(\hat{\mathfrak{s l}}_{2}\right)$, the existence of such exact sequences was known by [CP1]. Later this conjecture was proved for $U_{q}(\hat{\mathfrak{g}})$ by [N3], [Her1] (see Theorem 2.8(2)).

It is useful to introduce the rings associated with $\mathbb{T}\left(X_{r}\right)$.

Definition 2.3. The unrestricted T-algebra $\mathcal{T}\left(X_{r}\right)$ of type $X_{r}$ is the ring with generators $T_{m}^{(a)}(u)^{ \pm 1}(a \in I, m \in \mathbb{N}, u \in U)$ and the relations $\mathbb{T}\left(X_{r}\right)$. (Here we also assume the relation $T_{m}^{(a)}(u) T_{m}^{(a)}(u)^{-1}=1$ implicitly. We do not repeat this remark in the forthcoming similar definitions.) Also, we define the ring $\mathcal{T}^{\circ}\left(X_{r}\right)$ as the subring of $\mathcal{T}\left(X_{r}\right)$ generated by $T_{m}^{(a)}(u)(a \in$ $I, m \in \mathbb{N}, u \in U)$. 
We write all the relations in $\mathbb{T}\left(X_{r}\right)$ in a unified manner as follows:

$$
T_{m}^{(a)}\left(u-\frac{1}{t_{a}}\right) T_{m}^{(a)}\left(u+\frac{1}{t_{a}}\right)=T_{m-1}^{(a)}(u) T_{m+1}^{(a)}(u)+M_{m}^{(a)}(u),
$$

where $M_{m}^{(a)}(u)$ is the second term of the right-hand side of each relation, and $t_{a}$ is the number in (2.1). Then, define $S_{a m u}(T) \in \mathbb{Z}[T](a \in I, m \in \mathbb{N}, u \in U)$ by

$$
S_{a m u}(T)=T_{m}^{(a)}\left(u-\frac{1}{t_{a}}\right) T_{m}^{(a)}\left(u+\frac{1}{t_{a}}\right)-T_{m-1}^{(a)}(u) T_{m+1}^{(a)}(u)-M_{m}^{(a)}(u)
$$

so that all the relations in $\mathbb{T}\left(X_{r}\right)$ are written in the form $S_{a m u}(T)=0$. Let $I\left(\mathbb{T}\left(X_{r}\right)\right)$ denote the ideal of $\mathbb{Z}[T]$ generated by $S_{a m u}(T)$ 's. We consider the natural embedding $\mathbb{Z}[T] \subset \mathbb{Z}\left[T^{ \pm 1}\right]$.

We use the following description of the ring $\mathcal{T}^{\circ}\left(X_{r}\right)$.

LEMma 2.4. (1) There is a ring isomorphism:

$$
\mathcal{T}^{\circ}\left(X_{r}\right) \simeq \mathbb{Z}[T] /\left(\mathbb{Z}\left[T^{ \pm 1}\right] I\left(\mathbb{T}\left(X_{r}\right)\right) \cap \mathbb{Z}[T]\right) .
$$

(2) For $P(T) \in \mathbb{Z}[T]$, the following conditions are equivalent:

(i) $P(T) \in \mathbb{Z}\left[T^{ \pm 1}\right] I\left(\mathbb{T}\left(X_{r}\right)\right)$.

(ii) There is a nonzero monomial $M(T) \in \mathbb{Z}[T]$ such that $M(T) P(T) \in$ $I\left(\mathbb{T}\left(X_{r}\right)\right)$.

Let us clarify the relation between the ring $\mathcal{T}^{\circ}\left(X_{r}\right)$ and the Grothendieck ring $\operatorname{Rep} U_{q}(\hat{\mathfrak{g}})$ of the category of the type 1 finite-dimensional $U_{q}(\hat{\mathfrak{g}})$-modules [CP2].

Choose $\hbar \in \mathbb{C} \backslash 2 \pi \sqrt{-1} \mathbb{Q}$ arbitrarily. We set the deformation parameter $q$ of $U_{q}(\hat{\mathfrak{g}})$ as $q=e^{\hbar} \in \mathbb{C}^{\times}$, so that $q$ is not a root of unity.

Let

$$
\chi_{q}: \operatorname{Rep} U_{q}(\hat{\mathfrak{g}}) \rightarrow \mathbb{Z}\left[Y_{i, a}^{ \pm 1}\right]_{i \in I, a \in \mathbb{C}^{\times}}
$$

be the $q$-character map of $U_{q}(\hat{\mathfrak{g}})$ in $[\mathrm{FR}],[\mathrm{FM}]$, which is an injective ring homomorphism as shown in $[\mathrm{FR}]$. From now on, we employ the parameterization of the variables $Y_{a, q^{t u}}\left(a \in I, u \in \mathbb{C}_{t \hbar}\right)$ instead of $Y_{i, a}\left(i \in I, a \in \mathbb{C}^{\times}\right)$ in $[\mathrm{FR}],[\mathrm{FM}]$, where $t$ is the number in $(2.1)$.

The $q$-character ring $\operatorname{Ch} U_{q}(\hat{\mathfrak{g}})$ of $U_{q}(\hat{\mathfrak{g}})$ is defined to be $\operatorname{Im} \chi_{q}$. Thus, $\operatorname{Ch} U_{q}(\hat{\mathfrak{g}})$ is an integral domain and isomorphic to $\operatorname{Rep} U_{q}(\hat{\mathfrak{g}})$. 
Definition 2.5. A Kirillov-Reshetikhin module $W_{m}^{(a)}(u)(a \in I, m \in \mathbb{N}$, $\left.u \in \mathbb{C}_{t \hbar}\right)$ of $U_{q}(\hat{\mathfrak{g}})$ is the irreducible finite dimensional $U_{q}(\hat{\mathfrak{g}})$-module with highest weight monomial

$$
m_{+}=\prod_{j=1}^{m} Y_{a, q^{t u} q_{a}^{m+1-2 j}}
$$

where $q_{a}=q^{t / t_{a}}$. Especially, $W_{1}^{(a)}(u)\left(a \in I, u \in \mathbb{C}_{t \hbar}\right)$ is called a fundamental module. [Her1].

REMARK 2.6. The above $W_{m}^{(a)}(u)$ corresponds to $W_{m, q^{t u} q_{a}^{-m+1}}^{(a)}$ in [N3],

The following fact is well known.

Theorem 2.7. (Frenkel and Reshetikhin [FR, Corollary 2]) The ring $\mathrm{Ch} U_{q}(\hat{\mathfrak{g}})$ is freely generated by the fundamental characters $\chi_{q}\left(W_{1}^{(a)}(u)\right)(a \in$ $\left.I, u \in \mathbb{C}_{t \hbar}\right)$.

Correspondingly, we choose the domain $U$ of the parameter $u$ for the T-system $\mathbb{T}\left(X_{r}\right)$ as $U=\mathbb{C}_{t \hbar}$. Here is an alternative description of $\operatorname{Ch} U_{q}(\hat{\mathfrak{g}})$ by the $q$-characters of the Kirillov-Reshetikhin modules and the T-system $\mathbb{T}\left(X_{r}\right)$.

Theorem 2.8. Let $\widetilde{T}=\left\{\widetilde{T}_{m}^{(a)}(u):=\chi_{q}\left(W_{m}^{(a)}(u)\right) \mid a \in I, m \in \mathbb{N}, u \in \mathbb{C}_{t \hbar}\right\}$ be the family of the $q$-characters of the Kirillov-Reshetikhin modules of $U_{q}(\hat{\mathfrak{g}})$. Then, we have the following.

(1) The family $\widetilde{T}$ generates the ring $\operatorname{Ch} U_{q}(\hat{\mathfrak{g}})$.

(2) (Nakajima [N3], Hernandez [Her1]) The family $\widetilde{T}$ satisfies the $T$ system $\mathbb{T}\left(X_{r}\right)$ in $\operatorname{Ch} U_{q}(\hat{\mathfrak{g}})$ (by replacing $T_{m}^{(a)}(u)$ in $\mathbb{T}\left(X_{r}\right)$ with $\widetilde{T}_{m}^{(a)}(u)$ ).

(3) For any $P(T) \in \mathbb{Z}[T]$, the relation $P(\widetilde{T})=0$ holds in $\operatorname{Ch} U_{q}(\hat{\mathfrak{g}})$ if and only if there is a nonzero monomial $M(T) \in \mathbb{Z}[T]$ such that $M(T) P(T) \in$ $I\left(\mathbb{T}\left(X_{r}\right)\right)$.

Proof. (1) This is a corollary of Theorem 2.7 .

(2) This was proved by [N3, Theorem 1.1] (for simply laced case) and by [Her1, Theorem 3.4] (including nonsimply laced case).

(3) The "if" part follows from (2) and from the fact that $\mathrm{Ch} U_{q}(\hat{\mathfrak{g}})$ is an integral domain. Let us show the "only if" part. To begin with, we introduce 
the height of $T_{m}^{(a)}(u), \operatorname{ht} T_{m}^{(a)}(u)$, by

$$
\begin{aligned}
\text { for simply laced } X_{r}, & \text { ht } T_{m}^{(a)}(u)=m, \\
\text { for } X_{r}=B_{r}, & \operatorname{ht} T_{m}^{(a)}(u)= \begin{cases}2 m-1 & a=1, \ldots, r-1, \\
m & a=r,\end{cases} \\
\text { for } X_{r}=F_{4}, & \operatorname{ht} T_{m}^{(a)}(u)= \begin{cases}m & a=1, \ldots, r-1, \\
2 m-1 & a=r,\end{cases} \\
\text { for } X_{r}=C_{r}, & \operatorname{ht} T_{m}^{(a)}(u)= \begin{cases}2 m-1 & a=1,2, \\
m & a=3,4,\end{cases} \\
\text { for } X_{r}=G_{2}, & \operatorname{ht} T_{m}^{(a)}(u)= \begin{cases}3 m-2 & a=1, \\
m & a=2 .\end{cases}
\end{aligned}
$$

Then, the following facts can be easily checked by inspection:

(a) $\operatorname{ht} T_{m}^{(a)}(u)=1$ if and only if $m=1$;

(b) the variable $T_{m}^{(a)}(u)(m \geq 2)$ occurs in $S_{a, m-1, u}(T)$, and $h t T_{m}^{(a)}(u)$ is greater than the heights of the other variables occurring in $S_{a, m-1, u}(T)$.

For a polynomial $P(T) \in \mathbb{Z}[T]$, we define ht $P(T)$ by the greatest height of all the generators $T_{m}^{(a)}(u)$ occurring in $P(T)$.

Now suppose that there is a nontrivial relation $P(\widetilde{T})=0$ in $\operatorname{Ch} U_{q}(\hat{\mathfrak{g}})$ for $P(T) \in \mathbb{Z}[T]$, and that $h:=\operatorname{ht} P(T) \geq 2$. Let $S$ be the set of all the triplets $(a, m, u)$ such that $T_{m}^{(a)}(u)$ is of height $h$ and occurs in $P(T)$. Let $M_{h}(T)=\prod_{(a, m, u) \in S} T_{m-2}^{(a)}(u)$. Then, thanks to (a) and (b), there is some $Q(T) \in \mathbb{Z}[T]$ with ht $Q(T)<h$ such that $Q(T) \equiv M_{h}(T) P(T) \bmod I\left(\mathbb{T}\left(X_{r}\right)\right)$. Furthermore, by $(2), Q(\widetilde{T})=0$ in $\operatorname{Ch} U_{q}(\hat{\mathfrak{g}})$. Repeat it until the relation reduces to the form $Q(\widetilde{T})=0$ with ht $Q(T)=1$ or 0 . However, the former does not occur, since it contradicts Theorem 2.7. Therefore, we have ht $Q(T)=0$, that is, $Q(T)=0$, which proves the claim.

Corollary 2.9. The ring $\mathcal{T}^{\circ}\left(X_{r}\right)$ with $U=\mathbb{C}_{t \hbar}$ is isomorphic to $\operatorname{Rep} U_{q}(\hat{\mathfrak{g}})$ by the correspondence $T_{m}^{(a)}(u) \mapsto W_{m}^{(a)}(u)$.

Proof. It follows from Theorem 2.8 and Lemma 2.4 that

$$
\operatorname{Rep} U_{q}(\hat{\mathfrak{g}}) \simeq \operatorname{Ch} U_{q}(\hat{\mathfrak{g}}) \simeq \mathbb{Z}[T] /\left(\mathbb{Z}\left[T^{ \pm 1}\right] I\left(\mathbb{T}\left(X_{r}\right)\right) \cap \mathbb{Z}[T]\right) \simeq \mathcal{T}^{\circ}\left(X_{r}\right)
$$


It should be proved in the same way, using the character by [Kn], that $\mathcal{T}^{\circ}\left(X_{r}\right)$ with $U=\mathbb{C}$ is isomorphic to $\operatorname{Rep} Y(\mathfrak{g})$. In Appendix A.1 we give parallel results for the ring associated with the Q-system and $\operatorname{Rep} U_{q}(\mathfrak{g})$.

\subsection{Unrestricted Y-systems}

Definition 2.10. The unrestricted $Y$-system $\mathbb{Y}\left(X_{r}\right)$ of type $X_{r}$ is the following system of relations for a family of variables $Y=\left\{Y_{m}^{(a)}(u) \mid a \in\right.$ $I, m \in \mathbb{N}, u \in U\}$, where $Y_{m}^{(0)}(u)=Y_{0}^{(a)}(u)^{-1}=0$ if they occur in the righthand sides in the following relations.

For simply laced $X_{r}$,

$$
Y_{m}^{(a)}(u-1) Y_{m}^{(a)}(u+1)=\frac{\prod_{b \in I: C_{a b}=-1}\left(1+Y_{m}^{(b)}(u)\right)}{\left(1+Y_{m-1}^{(a)}(u)^{-1}\right)\left(1+Y_{m+1}^{(a)}(u)^{-1}\right)}
$$

For $X_{r}=B_{r}$,

$$
Y_{m}^{(a)}(u-1) Y_{m}^{(a)}(u+1)=\frac{\left(1+Y_{m}^{(a-1)}(u)\right)\left(1+Y_{m}^{(a+1)}(u)\right)}{\left(1+Y_{m-1}^{(a)}(u)^{-1}\right)\left(1+Y_{m+1}^{(a)}(u)^{-1}\right)}
$$

$$
\begin{aligned}
&\left.\begin{array}{c}
(1 \\
+
\end{array} Y_{m}^{(r-2)}(u)\right) \\
& \times\left(1+Y_{2 m-1}^{(r)}(u)\right)\left(1+Y_{2 m+1}^{(r)}(u)\right) \\
& Y_{m}^{(r-1)}(u-1) Y_{m}^{(r-1)}(u+1)=\frac{}{\times\left(1+Y_{2 m}^{(r)}\left(u-\frac{1}{2}\right)\right)\left(1+Y_{2 m}^{(r)}\left(u+\frac{1}{2}\right)\right)} \\
&\left(1+Y_{m-1}^{(r-1)}(u)^{-1}\right)\left(1+Y_{m+1}^{(r-1)}(u)^{-1}\right)
\end{aligned}
$$

For $X_{r}=C_{r}$,

$$
\begin{aligned}
Y_{m}^{(a)}\left(u-\frac{1}{2}\right) Y_{m}^{(a)}\left(u+\frac{1}{2}\right) & =\frac{\left(1+Y_{m}^{(a-1)}(u)\right)\left(1+Y_{m}^{(a+1)}(u)\right)}{\left(1+Y_{m-1}^{(a)}(u)^{-1}\right)\left(1+Y_{m+1}^{(a)}(u)^{-1}\right)} \\
\quad(1 \leq a \leq r-2) & \\
Y_{2 m}^{(r-1)}\left(u-\frac{1}{2}\right) Y_{2 m}^{(r-1)}\left(u+\frac{1}{2}\right) & =\frac{\left(1+Y_{2 m}^{(r-2)}(u)\right)\left(1+Y_{m}^{(r)}(u)\right)}{\left(1+Y_{2 m-1}^{(r-1)}(u)^{-1}\right)\left(1+Y_{2 m+1}^{(r-1)}(u)^{-1}\right)}
\end{aligned}
$$




$$
\begin{gathered}
Y_{2 m+1}^{(r-1)}\left(u-\frac{1}{2}\right) Y_{2 m+1}^{(r-1)}\left(u+\frac{1}{2}\right)=\frac{1+Y_{2 m+1}^{(r-2)}(u)}{\left(1+Y_{2 m}^{(r-1)}(u)^{-1}\right)\left(1+Y_{2 m+2}^{(r-1)}(u)^{-1}\right)} \\
Y_{m}^{(r)}(u-1) Y_{m}^{(r)}(u+1)=\frac{\left(1+Y_{2 m+1}^{(r-1)}(u)\right)\left(1+Y_{2 m-1}^{(r-1)}(u)\right)}{\left(1+Y_{2 m}^{(r-1)}\left(u-\frac{1}{2}\right)\right)\left(1+Y_{2 m}^{(r-1)}\left(u+\frac{1}{2}\right)\right)} \\
\left(1+Y_{m-1}^{(r)}(u)^{-1}\right)\left(1+Y_{m+1}^{(r)}(u)^{-1}\right)
\end{gathered}
$$

For $X_{r}=F_{4}$,

$$
\begin{aligned}
& Y_{m}^{(1)}(u-1) Y_{m}^{(1)}(u+1)=\frac{1+Y_{m}^{(2)}(u)}{\left(1+Y_{m-1}^{(1)}(u)^{-1}\right)\left(1+Y_{m+1}^{(1)}(u)^{-1}\right)}, \\
& \left(1+Y_{m}^{(1)}(u)\right)\left(1+Y_{2 m-1}^{(3)}(u)\right) \\
& \times\left(1+Y_{2 m+1}^{(3)}(u)\right)\left(1+Y_{2 m}^{(3)}\left(u-\frac{1}{2}\right)\right) \\
& Y_{m}^{(2)}(u-1) Y_{m}^{(2)}(u+1)=\frac{\times\left(1+Y_{2 m}^{(3)}\left(u+\frac{1}{2}\right)\right)}{\left(1+Y_{m-1}^{(2)}(u)^{-1}\right)\left(1+Y_{m+1}^{(2)}(u)^{-1}\right)} \text {, } \\
& Y_{2 m}^{(3)}\left(u-\frac{1}{2}\right) Y_{2 m}^{(3)}\left(u+\frac{1}{2}\right)=\frac{\left(1+Y_{m}^{(2)}(u)\right)\left(1+Y_{2 m}^{(4)}(u)\right)}{\left(1+Y_{2 m-1}^{(3)}(u)^{-1}\right)\left(1+Y_{2 m+1}^{(3)}(u)^{-1}\right)}, \\
& Y_{2 m+1}^{(3)}\left(u-\frac{1}{2}\right) Y_{2 m+1}^{(3)}\left(u+\frac{1}{2}\right)=\frac{1+Y_{2 m+1}^{(4)}(u)}{\left(1+Y_{2 m}^{(3)}(u)^{-1}\right)\left(1+Y_{2 m+2}^{(3)}(u)^{-1}\right)}, \\
& Y_{m}^{(4)}\left(u-\frac{1}{2}\right) Y_{m}^{(4)}\left(u+\frac{1}{2}\right)=\frac{1+Y_{m}^{(3)}(u)}{\left(1+Y_{m-1}^{(4)}(u)^{-1}\right)\left(1+Y_{m+1}^{(4)}(u)^{-1}\right)} .
\end{aligned}
$$

For $X_{r}=G_{2}$,

$$
\begin{aligned}
(1 & \left.+Y_{3 m-2}^{(2)}(u)\right)\left(1+Y_{3 m+2}^{(2)}(u)\right) \\
& \times\left(1+Y_{3 m-1}^{(2)}\left(u-\frac{1}{3}\right)\right) \\
& \times\left(1+Y_{3 m-1}^{(2)}\left(u+\frac{1}{3}\right)\right) \\
& \times\left(1+Y_{3 m+1}^{(2)}\left(u-\frac{1}{3}\right)\right) \\
& \times\left(1+Y_{3 m+1}^{(2)}\left(u+\frac{1}{3}\right)\right) \\
& \times\left(1+Y_{3 m}^{(2)}\left(u-\frac{2}{3}\right)\right)\left(1+Y_{3 m}^{(2)}\left(u+\frac{2}{3}\right)\right) \\
& \times\left(1+Y_{3 m}^{(2)}(u)\right) \\
Y_{3 m}^{(2)}\left(u-\frac{1}{3}\right) Y_{3 m}^{(2)}\left(u+\frac{1}{3}\right)=\frac{\left(1+Y_{m-1}^{(1)}(u)^{-1}\right)\left(1+Y_{m+1}^{(1)}(u)^{-1}\right)}{(1+1) Y_{m}^{(1)}(u+1)=} & \left.Y_{3 m-1}^{(2)}(u)^{-1}\right)\left(1+Y_{3 m+1}^{(2)}(u)^{-1}\right)
\end{aligned}
$$




$$
\begin{aligned}
& Y_{3 m+1}^{(2)}\left(u-\frac{1}{3}\right) Y_{3 m+1}^{(2)}\left(u+\frac{1}{3}\right)=\frac{1}{\left(1+Y_{3 m}^{(2)}(u)^{-1}\right)\left(1+Y_{3 m+2}^{(2)}(u)^{-1}\right)} \\
& Y_{3 m+2}^{(2)}\left(u-\frac{1}{3}\right) Y_{3 m+2}^{(2)}\left(u+\frac{1}{3}\right)=\frac{1}{\left(1+Y_{3 m+1}^{(2)}(u)^{-1}\right)\left(1+Y_{3 m+3}^{(2)}(u)^{-1}\right)}
\end{aligned}
$$

The choice of the domain $U=\mathbb{C}_{\xi}$ of the parameter $u$ effectively imposes an additional periodic condition:

$$
Y_{m}^{(a)}(u)=Y_{m}^{(a)}\left(u+\frac{2 \pi \sqrt{-1}}{\xi}\right) .
$$

Definition 2.11. The unrestricted $Y$-algebra $y\left(X_{r}\right)$ of type $X_{r}$ is the ring with generators $Y_{m}^{(a)}(u)^{ \pm 1},\left(1+Y_{m}^{(a)}(u)\right)^{-1}(a \in I, m \in \mathbb{N}, u \in U)$, and the relations $\mathbb{Y}\left(X_{r}\right)$.

The system $\mathbb{Y}\left(X_{r}\right)$ is introduced by $[\mathrm{KN}]$ (see also Remark 3.7 for the origin of the $Y$-systems).

Though the T-systems and Y-systems arose in different contexts with different motivations, there is a simple and remarkable connection between them, as described below. Recall that $M_{m}^{(a)}(u)$ is defined in (2.8).

THEOREM 2.12. (1) There is a ring homomorphism

$$
\varphi: y\left(X_{r}\right) \rightarrow \mathcal{T}\left(X_{r}\right)
$$

defined by

$$
Y_{m}^{(a)}(u) \mapsto \frac{M_{m}^{(a)}(u)}{T_{m-1}^{(a)}(u) T_{m+1}^{(a)}(u)},
$$

or, equivalently, by either of

$$
\begin{aligned}
1+Y_{m}^{(a)}(u) & \mapsto \frac{T_{m}^{(a)}\left(u-\frac{1}{t_{a}}\right) T_{m}^{(a)}\left(u+\frac{1}{t_{a}}\right)}{T_{m-1}^{(a)}(u) T_{m+1}^{(a)}(u)}, \\
1+Y_{m}^{(a)}(u)^{-1} & \mapsto \frac{T_{m}^{(a)}\left(u-\frac{1}{t_{a}}\right) T_{m}^{(a)}\left(u+\frac{1}{t_{a}}\right)}{M_{m}^{(a)}(u)},
\end{aligned}
$$

where $T_{0}^{(a)}(u)=1$. 
(2) There is a ring homomorphism

$$
\psi: \mathcal{T}\left(X_{r}\right) \rightarrow y\left(X_{r}\right)
$$

such that $\psi \circ \varphi=\operatorname{id}_{y}\left(X_{r}\right)$.

(3) $\mathrm{y}\left(X_{r}\right)$ is isomorphic to a subring and a quotient ring of $\mathcal{T}\left(X_{r}\right)$.

The homomorphism $\varphi$ is canonical, while $\psi$ is neither unique nor canonical.

Proof. Statement (3) is a corollary of (1) and (2); we prove (1) and (2). Here, we concentrate on the case $U=\mathbb{C}$. The modification of the proof for the case $U=\mathbb{C}_{\xi}$ is straightforward.

(i) The case $X_{r}$ is simply laced. (1) For simplicity, let us write the image $\varphi\left(Y_{m}^{(a)}(u)\right)$ as $Y_{m}^{(a)}(u)$. Then, the relation (2.15) is shown as follows:

$$
\begin{aligned}
Y_{m}^{(a)}(u-1) Y_{m}^{(a)}(u+1) & \frac{\prod_{b: C_{a b}=-1} T_{m}^{(b)}(u-1) T_{m}^{(b)}(u+1)}{T_{m-1}^{(a)}(u-1) T_{m+1}^{(a)}(u-1) T_{m-1}^{(a)}(u+1) T_{m+1}^{(a)}(u+1)} \\
= & \frac{\prod_{b: C_{a b}=-1}\left(T_{m-1}^{(b)}(u) T_{m+1}^{(b)}(u)+\prod_{c: C_{b c}=-1} T_{m}^{(c)}(u)\right)}{T_{m-2}^{(a)}(u) T_{m}^{(a)}(u)+\prod_{b: C_{a b}=-1} T_{m-1}^{(b)}(u)} \\
& \times \frac{1}{T_{m}^{(a)}(u) T_{m+2}^{(a)}(u)+\prod_{b: C_{a b}=-1} T_{m+1}^{(b)}(u)} \\
= & \frac{\prod_{b: C_{a b}=-1}\left(1+Y_{m}^{(b)}(u)\right)}{\left(1+Y_{m-1}^{(a)}(u)^{-1}\right)\left(1+Y_{m+1}^{(a)}(u)^{-1}\right)} .
\end{aligned}
$$

We remark that the above calculation is valid also at $m=1$ by formally setting $T_{-1}^{(0)}(u)=0$.

(2) Below we define the image $\psi\left(T_{m}^{(a)}(u)\right)(a \in I, m \in \mathbb{N}, u \in \mathbb{C})$ in three steps and then show that they satisfy $\mathbb{T}\left(X_{r}\right)$. For simplicity, we write the image $\psi\left(T_{m}^{(a)}(u)\right)$ as $T_{m}^{(a)}(u)$.

Step 1. We arbitrarily choose $T_{1}^{(a)}(u) \in \mathrm{y}\left(X_{r}\right)^{\times}(a \in I)$ for each $u \in \mathbb{C}$ in the region $-1 \leq \operatorname{Re} u<1$.

Step 2. We define $T_{1}^{(a)}(u)(a \in I)$ for the rest of the region $-2 \leq \operatorname{Re} u<2$ by

$$
T_{1}^{(a)}(u \pm 1)=\left(1+Y_{1}^{(a)}(u)^{-1}\right) \frac{M_{1}^{(a)}(u)}{T_{1}^{(a)}(u \mp 1)} .
$$


We repeat it to define $T_{1}^{(a)}(u)(a \in I)$ for all $u \in \mathbb{C}$.

Step 3. For each $a$, we recursively define $T_{m}^{(a)}(u)(m \geq 2, u \in \mathbb{C})$ by

$$
T_{m+1}^{(a)}(u)=\frac{1}{1+Y_{m}^{(a)}(u)} \frac{T_{m}^{(a)}(u-1) T_{m}^{(a)}(u+1)}{T_{m-1}^{(a)}(u)}
$$

where $T_{0}^{(a)}(u)=1$.

Claim. The family $T$ defined above satisfies the following relations in $y\left(X_{r}\right)$ :

$$
\begin{aligned}
1+Y_{m}^{(a)}(u) & =\frac{T_{m}^{(a)}(u-1) T_{m}^{(a)}(u+1)}{T_{m-1}^{(a)}(u) T_{m+1}^{(a)}(u)}, \\
1+Y_{m}^{(a)}(u)^{-1} & =\frac{T_{m}^{(a)}(u-1) T_{m}^{(a)}(u+1)}{M_{m}^{(a)}(u)} .
\end{aligned}
$$

The relation (2.29) clearly holds by (2.28). The relation (2.30) is shown by the induction on $m$, where the $m=1$ case is true by (2.27).

Now, taking the inverse sum of (2.29) and (2.30), we obtain (2.2). Therefore, $\psi$ is a ring homomorphism. Furthermore, taking the ratio of (2.29) and $(2.30)$, we obtain $Y_{m}^{(a)}(u)=M_{m}^{(a)}(u) /\left(T_{m-1}^{(a)}(u) T_{m+1}^{(a)}(u)\right)$. This proves $\psi \circ \varphi=\operatorname{id}_{y\left(X_{r}\right)}$.

(ii) The case $X_{r}$ is nonsimply laced. (1) This can be proved one by one with similar calculations to (2.26), though they are slightly more complicated.

(2) Below we define the image $\psi\left(T_{m}^{(a)}(u)\right)(a \in I, m \in \mathbb{N}, u \in \mathbb{C})$ in three steps and then show that they satisfy $\mathbb{T}\left(X_{r}\right)$. For simplicity, we again write the image $\psi\left(T_{m}^{(a)}(u)\right)$ as $T_{m}^{(a)}(u)$.

Step 1. First, we arbitrarily choose $T_{1}^{(a)}(u) \in \mathrm{y}\left(X_{r}\right)^{\times}(a \in I)$ for each $u \in \mathbb{C}$ in the region $-1 / t_{a} \leq \operatorname{Re} u<1 / t_{a}$. Next, for each $a$ with $t_{a}=2$ (resp. $t_{a}=3$, which occurs only for $X_{r}=G_{2}$ and $a=2$ ), we define $T_{2}^{(a)}(u)$ (resp. $T_{2}^{(a)}(u)$ and $\left.T_{3}^{(a)}(u)\right)$ in the region $-1 / t_{a} \leq \operatorname{Re} u<1 / t_{a}$ by

$$
T_{m+1}^{(a)}(u)=\frac{M_{m}^{(a)}(u)}{Y_{m}^{(a)}(u) T_{m-1}^{(a)}(u)}
$$

or, more explicitly,

$$
T_{2}^{(a)}(u)=\frac{T_{1}^{(a-1)}(u) T_{1}^{(a+1)}(u)}{Y_{1}^{(a)}(u)}, \quad T_{3}^{(2)}(u)=\frac{T_{1}^{(1)}\left(u-\frac{1}{3}\right) T_{1}^{(1)}\left(u+\frac{1}{3}\right)}{Y_{2}^{(2)}(u) T_{1}^{(2)}(u)}
$$


where $T_{1}^{(0)}(u)=T_{1}^{(r+1)}(u)=1$.

Step 2. Let $t$ be the number in (2.1). First, we define $T_{1}^{(a)}(u)(a \in I)$ for the rest of the region $-\left(1 / t_{a}\right)-(1 / t) \leq \operatorname{Re} u<\left(1 / t_{a}\right)+(1 / t)$ by

$$
T_{1}^{(a)}\left(u \pm \frac{1}{t_{a}}\right)=\left(1+Y_{1}^{(a)}(u)^{-1}\right) \frac{M_{1}^{(a)}(u)}{T_{1}^{(a)}\left(u \mp \frac{1}{t_{a}}\right)} .
$$

Next, we define $T_{m}^{(a)}(u)\left(t_{a}=2,3 ; m=2, \ldots, t_{a}\right)$ for the rest of the region $-\left(1 / t_{a}\right)-(1 / t) \leq \operatorname{Re} u<\left(1 / t_{a}\right)+(1 / t)$ by $(2.31)$. We repeat it to define $T_{m}^{(a)}(u)\left(a \in I ; m=1, \ldots, t_{a}\right)$ for all $u \in \mathbb{C}$.

Step 3. For each $a$, we recursively define $T_{m}^{(a)}(u)\left(m>t_{a}, u \in \mathbb{C}\right)$ by

$$
T_{m+1}^{(a)}(u)=\frac{1}{1+Y_{m}^{(a)}(u)} \frac{T_{m}^{(a)}\left(u-\frac{1}{t_{a}}\right) T_{m}^{(a)}\left(u+\frac{1}{t_{a}}\right)}{T_{m-1}^{(a)}(u)},
$$

where $T_{0}^{(a)}(u)=1$.

Claim 1. The family $T$ defined above satisfies the following relations in $y\left(X_{r}\right)$ :

$$
\begin{aligned}
& 1+Y_{m}^{(a)}(u)=\frac{T_{m}^{(a)}\left(u-\frac{1}{t_{a}}\right) T_{m}^{(a)}\left(u+\frac{1}{t_{a}}\right)}{T_{m-1}^{(a)}(u) T_{m+1}^{(a)}(u)} \\
&\left(t_{a}=2,3 ; m=1, \ldots, t_{a}-1\right), \\
& 1+Y_{m}^{(a)}(u)^{-1}= \frac{T_{m}^{(a)}\left(u-\frac{1}{t_{a}}\right) T_{m}^{(a)}\left(u+\frac{1}{t_{a}}\right)}{M_{m}^{(a)}(u)} \\
&\left(t_{a}=2,3 ; m=2, \ldots, t_{a}\right) .
\end{aligned}
$$

The relation (2.35) for $m=1$ is an immediate consequence of (2.31) and (2.33). The relation (2.36) for $m=2$ is verified one by one. For $t_{a}=3,(2.35)$ for $m=2$ is an immediate consequence of (2.31) and (2.36) for $m=2$, and (2.36) for $m=3$ is verified by (2.31) and (2.33).

Claim 2. The family $T$ defined above satisfies the following relations in $y\left(X_{r}\right)$ for any $(a, m, u)$ :

$$
1+Y_{m}^{(a)}(u)=\frac{T_{m}^{(a)}\left(u-\frac{1}{t_{a}}\right) T_{m}^{(a)}\left(u+\frac{1}{t_{a}}\right)}{T_{m-1}^{(a)}(u) T_{m+1}^{(a)}(u)},
$$




$$
1+Y_{m}^{(a)}(u)^{-1}=\frac{T_{m}^{(a)}\left(u-\frac{1}{t_{a}}\right) T_{m}^{(a)}\left(u+\frac{1}{t_{a}}\right)}{M_{m}^{(a)}(u)} .
$$

The relation (2.37) holds for any $(a, m, u)$ because of (2.34) and (2.35). The relation (2.38) holds for $m=1, \ldots, t_{a}$ because of (2.33) and (2.36). Then, one can verify (2.38) by the induction on $m$ one by one.

The rest of the argument is the same as for the simply laced case.

REMARK 2.13. The transformation (2.22) first appeared in [KP] for the simplest case $X_{r}=A_{1}$ and was generalized in [KNS1] for general $X_{r}$. The analogous transformation plays an important role also in the approach by cluster algebras with coefficients [FZ4].

\subsection{Regular solutions of $\mathbf{T}$ - and $\mathrm{Y}$-systems}

In application, we usually consider solutions of $\mathbb{T}\left(X_{r}\right)$ and $\mathbb{Y}\left(X_{r}\right)$ in a particular ring.

DeFinition 2.14. Let $R$ be a ring.

(i) A family $T=\left\{T_{m}^{(a)}(u) \in R \mid a \in I, m \in \mathbb{N}, u \in U\right\}$ satisfying $\mathbb{T}\left(X_{r}\right)$ is called a solution of the T-system $\mathbb{T}\left(X_{r}\right)$ in $R$. We say a solution $T$ of $\mathbb{T}\left(X_{r}\right)$ in $R$ is regular if $T_{m}^{(a)}(u) \in R^{\times}$for any $(a, m, u)$.

(ii) A family $Y=\left\{Y_{m}^{(a)}(u) \in R \mid a \in I, m \in \mathbb{N}, u \in U\right\}$ satisfying $\mathbb{Y}\left(X_{r}\right)$ is called a solution of the $Y$-system $\mathbb{Y}\left(X_{r}\right)$ in $R$. We say a solution $Y$ of $\mathbb{Y}\left(X_{r}\right)$ in $R$ is regular if $Y_{m}^{(a)}(u), 1+Y_{m}^{(a)}(u) \in R^{\times}$for any $(a, m, u)$.

REMARK 2.15. Actually, any solution of $\mathbb{Y}\left(X_{r}\right)$ is regular because, for any $Y_{m}^{(a)}(u)$, there is a relation among $\mathbb{Y}\left(X_{r}\right)$ such that $\left(1+Y_{m}^{(a)}(u)^{-1}\right)^{-1}$ appears in the right-hand side. However, this is not always true for the restricted Y-system we shall discuss in Section 3. Therefore, it is convenient to introduce the above definition so that the unrestricted/restricted T-/Ysystems can be treated in a unified manner.

Clearly, there is a one-to-one correspondence between the regular solutions of $\mathbb{T}\left(X_{r}\right)$ (resp. $\left.\mathbb{Y}\left(X_{r}\right)\right)$ in $R$ and the ring homomorphisms $f: \mathcal{T}\left(X_{r}\right) \rightarrow$ $R$ (resp. $f: y\left(X_{r}\right) \rightarrow R$ ).

As a corollary of Theorem 2.12(2), we obtain the following.

Corollary 2.16. For any ring $R$, the map

$$
\varphi^{*}: \operatorname{Hom}\left(\mathcal{T}\left(X_{r}\right), R\right) \rightarrow \operatorname{Hom}\left(y\left(X_{r}\right), R\right)
$$


induced from the homomorphism $\varphi$ in (2.21), is surjective. Namely, for any regular solution $Y$ of $\mathbb{Y}\left(X_{r}\right)$ in $R$, there exists some regular solution $T$ of $\mathbb{T}\left(X_{r}\right)$ in $R$ such that $Y$ is expressed by $T$ as

$$
Y_{m}^{(a)}(u)=\frac{M_{m}^{(a)}(u)}{T_{m-1}^{(a)}(u) T_{m+1}^{(a)}(u)} .
$$

\section{$\S 3$. Restricted T- and $\mathrm{Y}$-systems and their periodicities}

In this section we state the main claims of the paper. We first introduce the restricted $\mathrm{T}$ - and Y-systems together with the associated algebras. Then, the conjectures and the results concerning their periodicity property are presented.

\subsection{Restricted T- and $\mathrm{Y}$-systems}

Let $t_{a}(a \in I)$ be the numbers in (2.1).

Definition 3.1. Fix an integer $\ell \geq 2$. The level $\ell$ restricted $T$-system $\mathbb{T}_{\ell}\left(X_{r}\right)$ of type $X_{r}$ (with the unit boundary condition) is the system of relations (2.2)-(2.6) naturally restricted to a family of variables $T=\left\{T_{m}^{(a)}(u) \mid\right.$ $\left.a \in I ; m=1, \ldots, t_{a} \ell-1 ; u \in U\right\}$, where $T_{m}^{(0)}(u)=T_{0}^{(a)}(u)=1$, and furthermore, $T_{t_{a} \ell}^{(a)}(u)=1$ (the unit boundary condition) if they occur in the righthand sides in the relations.

Definition 3.2. The level $\ell$ restricted T-algebra $\mathcal{T}_{\ell}\left(X_{r}\right)$ of type $X_{r}$ is the ring with generators $T_{m}^{(a)}(u)^{ \pm 1}\left(a \in I ; m=1, \ldots, t_{a} \ell-1 ; u \in U\right)$ and the relations $\mathbb{T}_{\ell}\left(X_{r}\right)$. Also, we define the ring $\mathcal{T}_{\ell}^{\circ}\left(X_{r}\right)$ as the subring of $\mathcal{T}_{\ell}\left(X_{r}\right)$ generated by $T_{m}^{(a)}(u)\left(a \in I ; m=1, \ldots, t_{a} \ell-1 ; u \in U\right)$.

REMARK 3.3. The notion of the level $\ell$ restriction originates from a class of solvable lattice model, called the level $\ell$ restricted solid-on-solid (RSOS) model associated with the R-matrix of $U_{q}(\hat{\mathfrak{g}})$ at a $2 t\left(h^{\vee}+\ell\right)$ th root of unity $[\mathrm{ABF}],[\mathrm{JMO}],[\mathrm{Pas}],[\mathrm{BR}]$. The level $\ell$ restricted $T$-system was introduced in [KNS1], where, instead of the condition $T_{t_{a} \ell}^{(a)}(u)=1$ above, a slightly weaker condition $T_{t_{a} \ell+1}^{(a)}(u)=0$ was imposed. We hope that no serious confusion occurs by referring to $\mathbb{T}_{\ell}\left(X_{r}\right)$ also as level $\ell$ restricted $T$-system for simplicity. We impose the unit boundary condition here to ensure the periodicity property we are going to discuss. (Actually, this is not the only choice of the boundary condition showing the periodicity, but we do not discuss this point in the paper.) 
Proposition 3.4. The ring $\mathcal{T}_{\ell}^{\circ}\left(X_{r}\right)$ is isomorphic to a quotient of $\mathcal{T}^{\circ}\left(X_{r}\right)$.

Proof. First we note that the ring $\mathcal{T}^{\circ}\left(X_{r}\right)$ is freely generated by $T_{1}^{(a)}(u)$ $(a \in I, u \in U)$. This is true for $U=\mathbb{C}_{t \hbar}$ by Theorem 2.7, and so it is true for any choice of $U$, since nontrivial relations exist only among $T_{m_{i}}^{\left(a_{i}\right)}\left(u_{i}\right)$ with $u_{i}-u_{j} \in \mathbb{R}$. So we have a ring homomorphism

$$
\pi_{\ell}: \mathcal{T}^{\circ}\left(X_{r}\right) \rightarrow \mathcal{T}_{\ell}^{\circ}\left(X_{r}\right)
$$

uniquely determined by the condition $\pi_{\ell}\left(T_{1}^{(a)}(u)\right)=T_{1}^{(a)}(u)(a \in I, u \in U)$. We claim that $\pi_{\ell}\left(T_{m}^{(a)}(u)\right)=T_{m}^{(a)}(u)$ for any $m=1, \ldots, t_{a} \ell-1$, from which the surjectivity of $\pi_{\ell}$ follows. The claim can be shown by the induction on the height of $T_{m}^{(a)}(u)$ in (2.13). Namely, suppose that the claim holds for any $T_{k}^{(b)}(v)$ such that ht $T_{k}^{(b)}(v)$ is smaller than ht $T_{m}^{(a)}(u)$. Let $S_{a m u}(T)$ be the one in (2.9). Then, $S_{a, m-1, u}(T)=0$ in $\mathcal{T}^{\circ}\left(X_{r}\right)$ and $\mathcal{T}_{\ell}^{\circ}\left(X_{r}\right)$; hence, $\pi_{\ell}\left(S_{a, m-1, u}(T)\right)=S_{a, m-1, u}(T)$ in $\mathcal{T}_{\ell}^{\circ}\left(X_{r}\right)$. The claim follows from this and the induction hypothesis.

Similarly, we define the following.

Definition 3.5. Fix an integer $\ell \geq 2$. The level $\ell$ restricted $Y$-system $\mathbb{Y}_{\ell}\left(X_{r}\right)$ of type $X_{r}$ is the system of relations (2.15)-(2.19) naturally restricted to a family of variables $Y=\left\{Y_{m}^{(a)}(u) \mid a \in I ; m=1, \ldots, t_{a} \ell-1 ; u \in U\right\}$, where $Y_{m}^{(0)}(u)=Y_{0}^{(a)}(u)^{-1}=0$, and furthermore, $Y_{t_{a} \ell}^{(a)}(u)^{-1}=0$ if they occur in the right-hand sides in the relations.

Definition 3.6. The level $\ell$ restricted $Y$-algebra $y_{\ell}\left(X_{r}\right)$ of type $X_{r}$ is the ring with generators $Y_{m}^{(a)}(u)^{ \pm 1},\left(1+Y_{m}^{(a)}(u)\right)^{-1}\left(a \in I ; m=1, \ldots, t_{a} \ell-1 ; u \in\right.$ $U)$ and the relations $\mathbb{Y}_{\ell}\left(X_{r}\right)$.

REMARK 3.7. The system $\mathbb{Y}_{\ell}\left(X_{r}\right)$ was introduced by [Z] for simply laced $X_{r}$ and $\ell=2$ to characterize the solutions of the thermodynamic Bethe ansatz equations for the factorizable scattering theories. Then, it was extended to the general case by $[\mathrm{KN}]$ based on the thermodynamic treatment of $[\mathrm{Ku}]$ (see also [KNS1, Appendix B]). For simply laced $X_{r}$, it was also given by [RTV] independently.

For any ring $R$, one can define the regular solutions of $\mathbb{T}_{\ell}\left(X_{r}\right)$ and $\mathbb{Y}_{\ell}\left(X_{r}\right)$ in $R$ in the same way as Definition 2.14. Again, they are identified with the elements in $\operatorname{Hom}\left(\mathcal{T}_{\ell}\left(X_{r}\right), R\right)$ and $\operatorname{Hom}\left(y_{\ell}\left(X_{r}\right), R\right)$.

The restrictions of $\mathrm{T}$-systems and $\mathrm{Y}$-systems are partly compatible in view of Theorem 2.12. Namely, we have the following. 
Proposition 3.8. The correspondence (2.22), with $T_{0}^{(a)}(u)=T_{t_{a} \ell}^{(a)}(u)=$ 1, defines a ring homomorphism

$$
\varphi_{\ell}: y_{\ell}\left(X_{r}\right) \rightarrow \mathcal{T}_{\ell}\left(X_{r}\right)
$$

Proof. Due to Theorem 2.12, we have only to check the compatibility between the boundary conditions, $T_{t_{a} \ell}^{(a)}(u)=1$ and $Y_{t_{a} \ell}^{(a)}(u)^{-1}=0$. For simply laced $X_{r}$, this can be seen by formally setting $T_{\ell+1}^{(a)}(u)=0$ at $m=\ell-1$ in (2.26). The nonsimply laced case is similar.

Unfortunately, the properties (2) and (3) in Theorem 2.12 do not necessarily hold for general $X_{r}$ and $\ell$.

EXAmple 3.9. (1) The case $X_{r}=A_{2}$ and $\ell=2$. Two systems,

$$
\begin{array}{r}
\mathbb{T}_{2}\left(A_{2}\right): T_{1}^{(1)}(u-1) T_{1}^{(1)}(u+1)=1+T_{1}^{(2)}(u), \\
T_{1}^{(2)}(u-1) T_{1}^{(2)}(u+1)=1+T_{1}^{(1)}(u), \\
\mathbb{Y}_{2}\left(A_{2}\right): Y_{1}^{(1)}(u-1) Y_{1}^{(1)}(u+1)=1+Y_{1}^{(2)}(u), \\
Y_{1}^{(2)}(u-1) Y_{1}^{(2)}(u+1)=1+Y_{1}^{(1)}(u),
\end{array}
$$

are identical; moreover, we have $\varphi_{2}: Y_{1}^{(1)}(u) \mapsto T_{1}^{(2)}(u), Y_{1}^{(2)}(u) \mapsto T_{1}^{(1)}(u)$. Thus, $\varphi_{2}$ is bijective.

(2) The case $X_{r}=A_{3}$ and $\ell=2$. We have $\varphi_{2}: Y_{1}^{(1)}(u) \mapsto T_{1}^{(2)}(u)$, $Y_{1}^{(3)}(u) \mapsto T_{1}^{(2)}(u)$. Thus, $\varphi_{2}$ is not injective.

(3) The case $X_{r}=C_{2}$ and $\ell=2$. We have $\varphi_{2}: Y_{1}^{(1)}(u) \mapsto T_{1}^{(2)}(u) / T_{2}^{(1)}(u)$, $Y_{3}^{(1)}(u) \mapsto T_{1}^{(2)}(u) / T_{2}^{(1)}(u)$. Thus, $\varphi_{2}$ is not injective.

However, at least for $A_{r}$, one can resolve this incompatibility by modifying the boundary condition of $\mathbb{T}_{\ell}\left(X_{r}\right)$ while keeping the periodicity (Proposition 5.9).

There are some isomorphisms among the restricted T-algebras or Yalgebras.

EXAMPLE 3.10. (Level-rank duality) The rings $\mathcal{T}_{\ell}\left(A_{r-1}\right)$ and $\mathcal{T}_{r}\left(A_{\ell-1}\right)$ are isomorphic under the correspondence $T_{m}^{(a)}(u) \leftrightarrow T_{a}^{(m)}(u)$. The rings $y_{\ell}\left(A_{r-1}\right)$ and $y_{r}\left(A_{\ell-1}\right)$ are isomorphic under the correspondence $Y_{m}^{(a)}(u) \leftrightarrow$ $Y_{a}^{(m)}(u)^{-1}$. 


\subsection{T- and $\mathrm{Y}$-systems with discrete spectral parameter}

So far, we assume that the spectral parameter $u$ takes values in $U=\mathbb{C}$ or $\mathbb{C}_{\xi}$. In the original context of T- and Y-systems, the analyticities of $T_{m}^{(a)}(u)$ and $Y_{m}^{(a)}(u)$ with respect to $u$ are of fundamental importance [Z], [KP], [KN], [RTV], [KNS2].

However, from the algebraic point of view, it is possible to discretize the parameter $u$ by choosing $U=(1 / t) \mathbb{Z}$, where $t$ is the number in $(2.1)$. There are at least two reasons why we are interested in such a discretization.

First, by regarding $u$ as "discretized time," the T- and Y-systems have their own interests as discrete dynamical systems. For example, $\mathbb{T}\left(A_{r}\right)$ is a discrete analogue of the Toda field equation and a particular case of the Hirota's bilinear difference equation [Hi1], [Hi2], [KOS], [KLWZ] (see [KLWZ] for more information).

Second, the periodicities of the restricted T- and Y-systems, which are the subjects of this paper, concern only the algebraic aspect of the $\mathrm{T}$ - and Y-systems; therefore, it is adequate to discuss the periodicities in discretized systems.

From here to the end of Section 8, we assume $U=(1 / t) \mathbb{Z}$ for all the T-systems and Y-systems.

\subsection{Periodicity conjecture for restricted $\mathbf{T}$ - and $\mathbf{Y}$-systems}

For $X_{r}$, let $h^{\vee}$ be the dual Coxeter number of $X_{r}$ as listed below:

\begin{tabular}{c|ccccccccc}
$X_{r}$ & $A_{r}$ & $B_{r}$ & $C_{r}$ & $D_{r}$ & $E_{6}$ & $E_{7}$ & $E_{8}$ & $F_{4}$ & $G_{2}$ \\
\hline$h^{\vee}$ & $r+1$ & $2 r-1$ & $r+1$ & $2 r-2$ & 12 & 18 & 30 & 9 & 4
\end{tabular}

For simply laced $X_{r}, h^{\vee}$ equals the Coxeter number $h$ of $X_{r}$.

Let $\omega$ be the involution on the set $I$ such that $\omega(a)=a$ except for the following cases (in our enumeration):

$$
\begin{array}{ll}
\omega(a)=r+1-a(a \in I) & X_{r}=A_{r}, \\
\omega(r-1)=r, \omega(r)=r-1 & X_{r}=D_{r}(r: \text { odd }), \\
\omega(1)=6, \omega(2)=5, \omega(5)=2, \omega(6)=1 & X_{r}=E_{6} .
\end{array}
$$

(Caution: for $X_{r}=D_{r}(r$ : even), $\omega(a)=a(a \in I)$.) The involution $\omega$ is related to the longest element $\omega_{0}$ in the Weyl group of type $X_{r}$ by $\omega_{0}\left(\alpha_{a}\right)=$ $-\alpha_{\omega(a)}[\mathrm{B}]$ (cf. [FZ3, Proposition 2.5]).

Now let us give the main claim of the paper. 
CONJECTURE 3.11. The following relations hold in $\mathcal{T}_{\ell}\left(X_{r}\right)$ :

(1) Half-periodicity: $T_{m}^{(a)}\left(u+h^{\vee}+\ell\right)=T_{t_{a} \ell-m}^{(\omega(a))}(u)$.

(2) Periodicity: $T_{m}^{(a)}\left(u+2\left(h^{\vee}+\ell\right)\right)=T_{m}^{(a)}(u)$.

We may sometimes refer to (2) also as full-periodicity in contrast to (1). Of course, the full-periodicity follows from the half-periodicity.

This is the counterpart of the (already conjectured and partially proved) periodicity property for the restricted Y-systems in various contexts; here we present it in a form parallel to Conjecture 3.11.

CONJECTURE 3.12. The following relations hold in $y_{\ell}\left(X_{r}\right)$.

(1) Half-periodicity: $Y_{m}^{(a)}\left(u+h^{\vee}+\ell\right)=Y_{t_{a} \ell-m}^{(\omega(a))}(u)$.

(2) Periodicity: $Y_{m}^{(a)}\left(u+2\left(h^{\vee}+\ell\right)\right)=Y_{m}^{(a)}(u)$.

REMARK 3.13. One can rephrase these periodicity properties as those of the regular solutions of the corresponding T- and Y-systems in an arbitrary ring $R$. For example, suppose that Conjecture 3.11(1) is true. Then, for any regular solution $T$ of $\mathbb{T}_{\ell}\left(X_{r}\right)$ in $R$, the equality $T_{m}^{(a)}\left(u+h^{\vee}+\ell\right)=T_{t_{a} \ell-m}^{(\omega(a))}(u)$ holds in $R$. The converse is also true by setting $R=\mathcal{T}_{\ell}\left(X_{r}\right)$. This remark will be applicable to any periodicity statement in the rest of the paper as well.

Let us summarize the known and/or related results on Conjectures 3.11 and 3.12 so far.

(i) Conjecture 3.12 was initially given by [Z] for simply laced $X_{r}$ and $\ell=2$ and then generalized by [RTV] for simply laced $X_{r}$ and $\ell \geq 2$ (including half-periodicity) and by [KNS1, Appendix B] for general $X_{r}$ and $\ell \geq 2$ (fullperiodicity). The points established so far are as follows:

(a) It was proved for $X_{r}=A_{r}$ and $\ell=2$ by Gliozzi and Tateo [GT] via three-dimensional geometry. The same case was also proved by Frenkel and Szenes [FS] with the explicit solution given.

(b) It was proved for simply laced $X_{r}$ and $\ell=2$ by Fomin and Zelevinsky [FZ3] via the cluster algebra method.

(c) It was proved for $X_{r}=A_{r}$ and $\ell \geq 2$ by Volkov [V] via the determinant method.

(d) The full-periodicity was proved for simply laced $X_{r}$ and $\ell>2$ by Keller [Kel2] via the cluster algebra/category method. 
We emphasize that the nonsimply laced Y-systems treated in [FZ3], [Kel2] are different from ours, and their nonsimply laced Y-systems are identified with certain reductions of our Y-systems associated with the twisted quantum affine algebras (see Remark 9.22). In particular, there has been no systematic result on Conjecture 3.12 for the nonsimply laced case so far. The same remark applies to the T-systems as well.

(ii) Conjecture 3.11 appeared in $[\mathrm{CGT}]$ for simply laced $X_{r}$, while the one for nonsimply laced $X_{r}$ seems new in the literature. The following related results are already known:

(a) For simply laced $X_{r}$, we will see that the ring $\mathcal{T}_{\ell}^{\circ}\left(X_{r}\right)$ is isomorphic to (a subring of) a certain cluster algebra. The periodicity property of the corresponding cluster algebra is known for $\ell=2$ (including halfperiodicity) by Fomin and Zelevinsky [FZ2], [FZ3] and for $\ell>2$ (fullperiodicity only) by [Kel2]. A more precise account will be given in Section 4.

(b) For $X_{r}=A_{r}$ and $\ell \geq 2$, Conjecture 3.11 follows from a more general theorem by Henriques [Hen] proved by the graph theoretical method. The same case was also proved essentially by [V] while proving Conjecture 3.12. We will give a detailed account of the latter method in Section 5 .

(iii) Though Conjectures 3.11 and 3.12 are tightly connected to each other in view of the map $\varphi_{\ell}$ in Proposition 3.8, one is not the consequence of the other, in general. However, at least for simply laced $X_{r}$, they are unified as the periodicity property of the $F$-polynomials of the corresponding cluster algebra with coefficients [FZ4].

REMARK 3.14. Recall that the choice $U=\mathbb{C}_{\xi}$ for the domain of the parameter $u$ of the unrestricted T-algebra $\mathcal{T}\left(X_{r}\right)$ imposes the period $2 \pi \sqrt{-1} / \xi$ in (2.7), where $\xi$ is taken from $\mathbb{C} \backslash 2 \pi \sqrt{-1} \mathbb{Q}$ to avoid the incompatibility with the relations $\mathbb{T}\left(X_{r}\right)$. The level $\ell$ restricted T-algebra $\mathcal{T}_{\ell}\left(X_{r}\right)$ has an additional period $2\left(h^{\vee}+\ell\right)$. This means the choice $\xi=\pi \sqrt{-1} /\left(h^{\vee}+\ell\right)$ is compatible with the relations $\mathbb{T}_{\ell}\left(X_{r}\right)$. In the context of the $q$-character, we made the identification $\xi=t \hbar$, where $q=e^{\hbar}$. Then, the above choice corresponds to $q=\exp \left(\pi \sqrt{-1} / t\left(h^{\vee}+\ell\right)\right)$; namely, $q$ is a primitive $2 t\left(h^{\vee}+\ell\right)$ th root of unity. This is natural in view of the origin of the level $\ell$ restriction in Remark 3.3. We make a further remark on the implication of the periodicity of $\mathcal{T}_{\ell}\left(X_{r}\right)$ for the $q$-character in Section 10 . 


\subsection{Summary of methods and results}

In the following, we will study and partially prove Conjecture 3.11 by three independent methods. This is a good point to outline the methods and the results.

1. Cluster algebra/category method applied to $\mathcal{T}_{\ell}\left(X_{r}\right)$ with simply laced $X_{r}$ (Section 4)

This is actually more than a method to prove Conjecture 3.11, since it includes the identification of the ring $\mathcal{T}_{\ell}^{\circ}\left(X_{r}\right)$ as a (subring of) cluster algebra.

In the simplest case $\ell=2$, the ring $\mathcal{T}_{2}^{\circ}\left(X_{r}\right)$ is isomorphic to the tensor square of the cluster algebra $\mathcal{A}_{Q}$ of type $X_{r}$ (Proposition 4.3). The ring $\mathcal{A}_{Q}$ is a cluster algebra of finite type and is particularly well studied. In particular, the periodicity property of $\mathcal{A}_{Q}$ is proved in [FZ2], [FZ3] by making use of the piecewise-linear modification of the simple reflections acting on the set of the almost positive roots $\Phi_{\geq-1}$ of type $X_{r}$. The periodicity of $\mathcal{T}_{2}\left(X_{r}\right)$ is its immediate corollary (Corollary 4.5).

For the case $\ell>2$, the ring $\mathcal{T}_{\ell}^{\circ}\left(X_{r}\right)$ is isomorphic to the tensor square of a subring of the cluster algebra $\mathcal{A}_{Q \square Q^{\prime}}$, where $Q \square Q^{\prime}$ is the square product of quivers recently introduced by [Kel2], [HL] (Proposition 4.24). The cluster algebra $\mathcal{A}_{Q \square Q^{\prime}}$ is not of finite type; nevertheless, it still admits the periodicity along the bipartite belt of [FZ4]. The periodicity of $\mathcal{A}_{Q \square Q^{\prime}}$ is studied in [Kel2], in a more general situation with coefficients, using the categorification by the 2-Calabi-Yau category associated with the tensor product of the path algebras of quivers $Q$ and $Q^{\prime}$. The full-periodicity of $\mathcal{T}_{\ell}\left(X_{r}\right)$ is its immediate corollary. Furthermore, this cluster categorical approach can be adapted for the half-periodicity. Thus, we obtain the desired periodicity for $\mathcal{T}_{\ell}\left(X_{r}\right)$ (Corollary 4.29).

2. Determinant method applied to $\mathcal{T}_{\ell}\left(A_{r}\right)$ and $\mathcal{T}_{\ell}\left(C_{r}\right)$ (Sections 5 and 6 )

The method seeks a manifestly periodic expression of $T_{m}^{(a)}(u)$ as a minor of a matrix $M$ over $\mathcal{T}_{\ell}\left(X_{r}\right)$ of infinitely many finite columns with periodicity. It was introduced by $[\mathrm{V}]$ to prove the periodicity of the regular solutions of $\mathbb{Y}_{\ell}\left(A_{r}\right)$ in $\mathbb{C}$.

Such a determinant expression (without periodicity) is known for the unrestricted T-system $\mathbb{T}\left(A_{r}\right)$ by [KLWZ, (2.25)], where the relation (2.2) of $\mathbb{T}\left(A_{r}\right)$ is regarded as the Hirota's bilinear difference equation. Then, the existence of such a determinant expression is viewed as a discrete analogue of the well-known relation between the Hirota's bilinear equation and the Grassmannians [S]. Remarkably, the restriction of the T-system to $\mathbb{T}_{\ell}\left(A_{r}\right)$ 
is compatible with this determinant expression by imposing the periodicity on the matrix $M$ (Proposition 5.8). This forces the desired periodicity for $\mathcal{T}_{\ell}\left(A_{r}\right)$ (Theorem 5.3).

Since the method takes advantage of the bilinearity of the relation of $\mathbb{T}\left(A_{r}\right)$, it does not seem applicable to $X_{r}$ other than $A_{r}$. A pleasant surprise is that it is still applicable for $C_{r}$ through the relation between $\mathcal{T}\left(C_{r}\right)$ and a certain variant of $\mathcal{T}\left(A_{2 r+1}\right)[\mathrm{KOSY}]$. (Note that this is different from the usual "folding" relation between $C_{r}$ and $A_{2 r-1}$.) This relation is compatible with the restriction and induces the relation between $\mathcal{T}_{\ell}\left(C_{r}\right)$ and $\widehat{\mathcal{T}}_{2 \ell}\left(A_{2 r+1}\right)$, where $\widehat{\mathcal{T}}_{2 \ell}\left(A_{2 r+1}\right)$ is a variant of $\mathcal{T}_{2 \ell}\left(A_{2 r+1}\right)$ (Proposition 6.8 ). Since $\widehat{\mathcal{T}}_{2 \ell}\left(A_{2 r+1}\right)$ admits the determinant expression, the desired periodicity for $\mathcal{T}_{\ell}\left(C_{r}\right)$ is obtained (Corollary 6.4). This is the first main result concerning Conjecture 3.11 for the nonsimply laced case.

At this moment the method is applicable only for these two cases, since a similar relation between $\mathcal{T}\left(X_{r}\right)$ and a certain variant of $\mathcal{T}\left(A_{r^{\prime}}\right)$ is not known for the other types $X_{r}$.

3. Direct method applied to $\mathcal{T}_{2}\left(A_{r}\right), \mathcal{T}_{2}\left(D_{r}\right)$, and $\mathcal{T}_{2}\left(B_{r}\right)$ (Section 7 )

The method seeks a manifestly periodic Laurent polynomial expression of $T_{m}^{(a)}(u)$ in terms of the "initial variables" by considering the T-system as a discrete dynamical system. At least for the above three cases, we can directly find such an expression with the aid of a computer and verify that it indeed satisfies the T-system.

The problem to express the cluster variables in terms of the initial cluster is a much-studied subject (e.g., [CC], [FZ4], [YZ]). The first two cases, $\mathcal{T}_{2}\left(A_{r}\right)$ and $\mathcal{T}_{2}\left(D_{r}\right)$, should be obtained as the specialization of those more general expressions. Our goal here is to prove the periodicity for $\mathcal{T}_{2}\left(B_{r}\right)$, which is the first nontrivial result for $B_{r}$. (Let us repeat that this is different from the tensor square of the cluster algebra of type $B_{r}$.)

\section{§4. Cluster algebra/category method: $\mathcal{T}_{\ell}\left(X_{r}\right)$ with simply laced $X_{r}$}

In this section, we study the periodicity of $\mathcal{T}_{\ell}\left(X_{r}\right)$ for simply laced $X_{r}$. We establish the relation between the $\operatorname{ring} \mathcal{T}_{\ell}^{\circ}\left(X_{r}\right)$ and cluster algebras [FZ1], [FZ2]. Then, the periodicity of $\mathcal{T}_{\ell}\left(X_{r}\right)$ reduces to that of the corresponding cluster algebra. For $\ell=2$, the periodicity of the corresponding cluster algebra is known by [FZ2], [FZ3]. For $\ell>2$, the full-periodicity of the corresponding cluster algebra is recently shown by [Kel2] using the cluster categorical method. We prove the half-periodicity for $\ell>2$ as well by adapting 
this categorical method (see [Kel2] for a comprehensive review of cluster algebras and cluster categories).

\subsection{Cluster algebra}

For a finite quiver $Q$ without loops or 2-cycles with vertex set, say, $I=$ $\{1, \ldots, n\}$ and an $I$-tuple of variables $x=\left\{x_{1}, \ldots, x_{n}\right\}$, we define a cluster algebra (with trivial coefficients) $\mathcal{A}_{Q}[\mathrm{FZ1}],[\mathrm{FZ2}]$, which is a $\mathbb{Z}$-subalgebra of the field $\mathbb{Q}\left(x_{1}, \ldots, x_{n}\right)$, as follows.

(1) We start from the pair ("initial seed") $(Q, x)$, where $Q$ and $x$ are as above.

(2) For each $k=1, \ldots, n$, we define another pair ("seed") $(R, y)=\mu_{k}(Q, x)$ of a quiver $R$ without loops or 2-cycles with vertex set $I$ and an $I$-tuple $y=\left\{y_{1}, \ldots, y_{n}\right\}, y_{i} \in \mathbb{Q}\left(x_{1}, \ldots, x_{n}\right)$, called the mutation of $(Q, x)$ at $k$, where $y$ is given by the exchange relation,

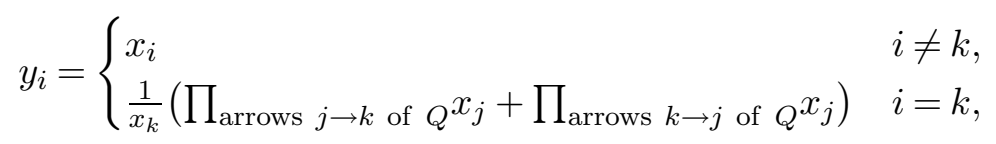

while $R=\mu_{k}(Q)$ is obtained from $Q$ by the following mutation rule.

(i) For each $i \rightarrow k \rightarrow j$ of $Q$, create a new arrow $i \rightarrow j$.

(ii) Replace each $i \rightarrow k$ of $Q$ with $k \rightarrow i$, and $k \rightarrow j$ of $Q$ with $j \rightarrow k$, respectively.

(iii) Remove a maximal disjoint collection of 2-cycles of the resulting quiver after (i) and (ii).

(3) Iterate the mutation for every new seed at every $k$ and collect all the (possibly infinite number of) seeds. For any seed $(R, y), y$ is called a cluster, and each element $y_{i}$ of $y$ is called a cluster variable.

(4) The cluster algebra $\mathcal{A}_{Q}$ is the $\mathbb{Z}$-subalgebra of the field $\mathbb{Q}\left(x_{1}, \ldots, x_{n}\right)$ generated by all the cluster variables.

Due to the Laurent phenomenon [FZ1], $\mathcal{A}_{Q}$ is a subring of $\mathbb{Z}\left[x_{1}^{ \pm 1}, \ldots, x_{n}^{ \pm 1}\right]$.

\subsection{Level 2 case}

Here we study the periodicity of $\mathcal{T}_{2}\left(X_{r}\right)$ for simply laced $X_{r}$. Since the case $X_{r}=A_{1}$ is trivial, we assume $X_{r} \neq A_{1}$.

Let $X_{r}\left(\neq A_{1}\right)$ be a simply laced Dynkin diagram, and let $I=I_{+} \sqcup I_{-}$be a bipartite decomposition of the vertex set $I$ of $X_{r}$; namely, $C_{a b}=0$ for any $a, b \in I_{ \pm}$with $a \neq b$. We set $\varepsilon(a)= \pm$ for $a \in I_{ \pm}$. 
Recall that the ring $\mathcal{T}_{2}\left(X_{r}\right)$ has the generators $T=\left\{T_{1}^{(a)}(u)^{ \pm 1} \mid a \in I, u \in\right.$ $\mathbb{Z}\}$ and the relations $\mathbb{T}_{2}\left(X_{r}\right)$ :

$$
T_{1}^{(a)}(u-1) T_{1}^{(a)}(u+1)=1+\prod_{b \in I: C_{a b}=-1} T_{1}^{(b)}(u) .
$$

Let $\mathcal{T}_{2}^{\circ}\left(X_{r}\right)_{ \pm}$be the subring of $\mathcal{T}_{2}^{\circ}\left(X_{r}\right)$ generated by $T_{1}^{(a)}(u) \quad(a \in I, u \in$ $\mathbb{Z})$ such that $\varepsilon(a)(-1)^{u}= \pm$, where we identify + and - with 1 and -1 , respectively. Since the relation $(4.2)$ closes among those $T_{1}^{(a)}(u)$ with fixed parity $\varepsilon(a)(-1)^{u}$, we have

$$
\mathcal{T}_{2}^{\circ}\left(X_{r}\right) \simeq \mathcal{T}_{2}^{\circ}\left(X_{r}\right)_{+} \otimes_{\mathbb{Z}} \mathcal{T}_{2}^{\circ}\left(X_{r}\right)_{-}, \quad \mathcal{T}_{2}^{\circ}\left(X_{r}\right)_{+} \simeq \mathcal{T}_{2}^{\circ}\left(X_{r}\right)_{-} .
$$

Let $Q=Q\left(X_{r}\right)$ be the alternating quiver such that $X_{r}$ is the underlying graph, $a \in I_{+}$is a source, and $a \in I_{-}$is a sink of $Q$. We introduce an $I$-tuple of variables $x=\left\{x_{a}\right\}_{a \in I}$, and we define $\mathcal{A}_{Q}$ to be the cluster algebra with initial seed $(Q, x)$.

Following [FZ2], [FZ3], we introduce composed mutations $\mu_{ \pm}=\prod_{a \in I_{ \pm}} \mu_{a}$ and $\mu=\mu_{-} \mu_{+}$for $\mathcal{A}_{Q}$. We set $x=x(0)$, and we define clusters $x(u)=$ $\left\{x_{a}(u)\right\}_{a \in I}(u \in \mathbb{Z})$ of $\mathcal{A}_{Q}$ by the following sequence of the mutations:

$$
\begin{aligned}
\cdots & \stackrel{\mu_{+}}{\longleftrightarrow}\left(Q^{\mathrm{op}}, x(-1)\right) \stackrel{\mu_{-}}{\longleftrightarrow}(Q, x(0)) \stackrel{\mu_{+}}{\longleftrightarrow}\left(Q^{\mathrm{op}}, x(1)\right) \\
& \stackrel{\mu_{-}}{\longleftrightarrow}(Q, x(2)) \stackrel{\mu_{+}}{\longleftrightarrow} \cdots,
\end{aligned}
$$

where $Q^{\text {op }}$ is the opposite quiver of $Q$, that is, the quiver obtained from $Q$ by reversing all the arrows. In particular,

$$
\begin{aligned}
x_{a}(u+1) & =x_{a}(u) & & \text { if } \varepsilon(a)(-1)^{u}=-, \\
(Q, x(2 k)) & =\mu^{k}(Q, x(0)) & & (k \in \mathbb{Z}), \\
\left(Q^{\text {op }}, x(2 k+1)\right) & =\mu_{+} \mu^{k}(Q, x(0)) & & (k \in \mathbb{Z}) .
\end{aligned}
$$

Furthermore, any cluster variable of $\mathcal{A}_{Q}$ occurs in $x(u)$ for some $u \in \mathbb{Z}$, due to [FZ2, Theorems 1.9 and 3.1]. (This is not true for a general finite quiver Q.)

Lemma 4.1 ([FZ4, (8.12)]). The family $\left\{x_{a}(u) \mid a \in I, u \in \mathbb{Z}\right\}$ satisfies the T-system $\mathbb{T}_{2}\left(X_{r}\right)$ in $\mathcal{A}_{Q} ;$ namely,

$$
x_{a}(u-1) x_{a}(u+1)=1+\prod_{b \in I: C_{a b}=-1} x_{b}(u) .
$$


Proof. For example, suppose that $a \in I_{+}$and that $u$ is odd. Then,

$$
x_{a}(u-1)=\mu_{+}\left(x_{a}(u)\right)=\frac{1}{x_{a}(u)}\left(1+\prod_{b \in I: C_{a b}=-1} x_{b}(u)\right)
$$

by (4.1), and $x_{a}(u)=x_{a}(u+1)$ by (4.5). The other cases are similar.

Now let us describe the relation between the rings, $\mathcal{T}_{2}\left(X_{r}\right)$ and $\mathcal{A}_{Q}$. Define a ring homomorphism $f: \mathcal{A}_{Q} \rightarrow \mathcal{T}_{2}\left(X_{r}\right)$ as the restriction of the ring homomorphism $\mathbb{Z}\left[x_{a}^{ \pm 1}\right]_{a \in I} \rightarrow \mathcal{T}_{2}\left(X_{r}\right)$ given by

$$
f: x_{a}^{ \pm 1} \mapsto \begin{cases}T_{1}^{(a)}(0)^{ \pm 1} & a \in I_{+}, \\ T_{1}^{(a)}(1)^{ \pm 1} & a \in I_{-} .\end{cases}
$$

Then, we have the following (see Figure 2).

Lemma 4.2. For the above homomorphism $f: \mathcal{A}_{Q} \rightarrow \mathcal{T}_{2}\left(X_{r}\right)$,

$$
f: x_{a}(u) \mapsto \begin{cases}T_{1}^{(a)}(u) & \varepsilon(a)(-1)^{u}=+, \\ T_{1}^{(a)}(u+1) & \varepsilon(a)(-1)^{u}=-.\end{cases}
$$

Proof. For $u=0,(4.10)$ holds by the definition of $f$. Then, one can prove (4.10) by the induction on $\pm u$ with (4.2), (4.5), and (4.7).

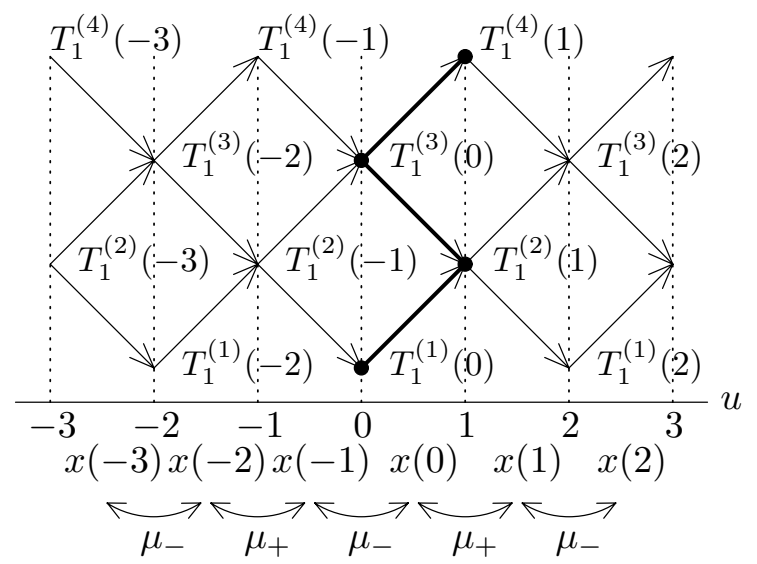

Figure 2: Relation of $x(u)$ and $T_{1}^{(a)}(u)$ for $X_{r}=A_{4}$. The thick quiver corresponds to the initial seed $(Q, x(0))$ of the cluster algebra $\mathcal{A}_{Q}$, where we take $I_{+}=\{1,3\}, I_{-}=\{2,4\}$. 
Proposition 4.3. The ring $\mathcal{T}_{2}^{\circ}\left(X_{r}\right)$ is isomorphic to $\mathcal{A}_{Q} \otimes_{\mathbb{Z}} \mathcal{A}_{Q}$.

Proof. It follows from Lemma 4.2 that the image $f\left(\mathcal{A}_{Q}\right)$ is $\mathcal{T}_{2}^{\circ}\left(X_{r}\right)_{+}$. Furthermore, the inverse correspondence $g: \mathcal{T}_{2}^{\circ}\left(X_{r}\right)_{+} \rightarrow \mathcal{A}_{Q}, T_{1}^{(a)}(u) \mapsto x_{a}(u)$ defines a homomorphism by Lemma 4.1 and the fact that $\mathcal{A}_{Q}$ is an integral domain. Therefore,

$$
\mathcal{T}_{2}^{\circ}\left(X_{r}\right)_{+} \simeq \mathcal{A}_{Q}
$$

and we obtain the assertion.

Thanks to the isomorphism, the periodicity of $\mathcal{T}_{2}\left(X_{r}\right)$ is reduced to the known periodicity of $\mathcal{A}_{Q}$. By the correspondence (4.10), it is easy to check that the periodicity of $\mathcal{T}_{2}\left(X_{r}\right)$ is translated as

$$
\begin{aligned}
& \text { half-periodicity: } \quad x_{a}(u+h+2)=x_{\omega(a)}(u), \\
& \text { periodicity: } \quad x_{a}(u+2(h+2))=x_{a}(u) .
\end{aligned}
$$

Recall that the Coxeter number $h=h^{\vee}$ of $X_{r}$ is odd if and only if $X_{r}=A_{r}$ $(r:$ even); furthermore, the involution $\omega: I \rightarrow I$ induces a quiver isomorphism $\omega: Q \rightarrow Q^{\text {op }}$ if $X_{r}=A_{r}$ ( $r:$ even) and $\omega: Q \rightarrow Q$ otherwise. For a pair of seeds $(R, y)$ and $\left(R^{\prime}, y^{\prime}\right)$, we write $(R, y) \stackrel{\nu}{=}\left(R^{\prime}, y^{\prime}\right)$ if $\nu: I \rightarrow I$ is a bijection that induces a quiver isomorphism $R \rightarrow R^{\prime}$ and $y_{\nu(a)}^{\prime}=y_{a}$ for any $a \in R$. The following periodicity of $\mathcal{A}_{Q}$ is due to [FZ2, Theorems 1.9 and $3.1]$ and [FZ3, Propositions 2.5 and 2.6].

Theorem 4.4 (Fomin and Zelevinsky [FZ2], [FZ3]). The following equalities hold for $\mathcal{A}_{Q}$ (u: even).

(1) Half-periodicity:

(i) for $X_{r}$ other than $A_{r}$ ( $r$ : even), where $h$ is even,

$$
\mu^{(h+2) / 2}(Q, x(u)) \stackrel{\omega}{=}(Q, x(u)) ;
$$

(ii) for $X_{r}=A_{r}$ ( $r$ : even), where $h$ is odd,

$$
\mu_{+} \mu^{(h+1) / 2}(Q, x(u)) \stackrel{\omega}{=}(Q, x(u)) .
$$

(2) Periodicity: For any $X_{r}$,

$$
\mu^{h+2}(Q, x(u)) \stackrel{\mathrm{id}}{=}(Q, x(u)) .
$$


COROLlary 4.5. The following relations hold in $\mathcal{T}_{2}\left(X_{r}\right)$ for any simply laced $X_{r}$.

(1) Half-periodicity: $T_{1}^{(a)}(u+h+2)=T_{1}^{(\omega(a))}(u)$.

(2) Periodicity: $T_{1}^{(a)}(u+2(h+2))=T_{1}^{(a)}(u)$.

Proof. The relations in (4.12) immediately follow from Theorem 4.4 and (4.6).

\subsection{Alternative proof of Theorem 4.4 by cluster category}

Here we present an alternative proof of Theorem 4.4 based on the categorification of $\mathcal{A}_{Q}$ by the cluster category $\mathcal{C}_{Q}$, in the spirit of [Kel2]. The definitions and results here will be also used to prove the periodicity for the levels greater than two in Section 4.5.

Let $Q$ be the alternating quiver whose underlying graph is simply laced $X_{r}$ other than $A_{1}$ as in Section 4.2 . Let $K$ be an algebraically closed field, and let $K Q$ be the path algebra of $Q$ [ARS], [ASS]. We denote by $\mathcal{D}_{Q}=\mathcal{D}^{\mathrm{b}}(\bmod K Q)$ the bounded derived category of finite dimensional $K Q$ modules. Then $\mathcal{D}_{Q}$ forms a $K$-linear triangulated category with the suspension functor [1]. We denote by $D$ the $K$-dual. The autoequivalence

$$
\tau:=D(K Q)[-1] \stackrel{\mathrm{L}}{\otimes}{ }_{K Q}-: \mathcal{D}_{Q} \rightarrow \mathcal{D}_{Q}
$$

is called the Auslander-Reiten translation and plays an important role in representation theory of $K Q$ [ARS], [ASS], [Ha].

Now we define another autoequivalence of $\mathcal{D}_{Q}$ by $F:=\tau^{-1} \circ[1]$. Then the cluster category of $Q$ [BMRRT] is defined as the orbit category

$$
\mathcal{C}_{Q}:=\mathcal{D}_{Q} / F
$$

which means that $\mathcal{C}_{Q}$ has the same objects with $\mathcal{D}_{Q}$, and the morphism space is given by

$$
\operatorname{Hom}_{\mathcal{C}_{Q}}(X, Y):=\bigoplus_{i \in \mathbf{Z}} \operatorname{Hom}_{\mathcal{D}_{Q}}\left(X, F^{i}(Y)\right)
$$

for any $X, Y \in \mathcal{C}_{Q}$. Then $\mathcal{C}_{Q}$ forms a triangulated category with the suspension functor [1], and the natural functor $\mathcal{D}_{Q} \rightarrow \mathcal{C}_{Q}$ is a triangle functor [Kel1].

For $a \in I$, we denote by $e_{a}$ the path of length zero in $Q$. Define $K Q$ modules by

$$
P_{a}:=(K Q) e_{a}, \quad P:=K Q=\bigoplus_{a \in I} P_{a}
$$


The following description of indecomposable objects in $\mathcal{C}_{Q}$ follows from Gabriel's theorem [ASS] and from Fomin and Zelevinsky's [FZ2] description of finite type cluster algebras.

THEOREM 4.6. There exists a bijection

$X:\left\{\right.$ indecomposable objects in $\left.\mathcal{C}_{Q}\right\} / \simeq \rightarrow\left\{\right.$ cluster variables in $\left.\mathcal{A}_{Q}\right\}$

satisfying $X_{P_{a}}=x_{a}(0)$ for any $a \in I$.

We say that an object $T=\bigoplus_{a \in I} T_{a} \in \mathcal{C}_{Q}$ is cluster tilting if

(1) each $T_{a}$ is indecomposable and mutually nonisomorphic, and

(2) $\operatorname{Hom}_{\mathcal{C}_{Q}}\left(T_{a}, T_{b}[1]\right)=0$ holds for any $a, b \in I$.

For a cluster tilting object $T \in \mathcal{C}_{Q}$, we denote by $Q_{T}$ the quiver of the endomorphism ring $\operatorname{End}_{\mathcal{C}_{Q}}(T)[\mathrm{ARS}],[\mathrm{ASS}]$.

We give two important examples of cluster tilting objects.

ExAmPLE 4.7. (1) The $K Q$-module

$$
P=\bigoplus_{a \in I} P_{a}
$$

gives a cluster tilting object. We have $Q_{P}=Q$, since $\operatorname{End}_{\mathcal{C}_{Q}}(P)=K Q$.

(2) We define $K Q$-modules by

$$
U_{a}:=\left\{\begin{array}{ll}
\tau^{-1}\left(P_{a}\right) & a \in I_{+}, \\
P_{a} & a \in I_{-},
\end{array} \quad U:=\bigoplus_{a \in I} U_{a} .\right.
$$

Then $U$ is a tilting $K Q$-module, so it gives a cluster tilting object in $\mathcal{C}_{Q}$. We have $Q_{U}=Q^{\mathrm{op}}$, since $\operatorname{End}_{\mathcal{D}_{Q}}(U) \simeq K Q^{\mathrm{op}}$.

The mutation of cluster tilting objects is introduced in [BMRRT, Theorem 5.1].

Theorem 4.8. Let $T=\bigoplus_{a \in I} T_{a} \in \mathcal{C}_{Q}$ be a cluster tilting object. For any $a \in I$, there exists a unique indecomposable object $T_{a}^{*} \in \mathcal{C}_{Q}$ which is not isomorphic to $T_{a}$ such that $\left(T / T_{a}\right) \oplus T_{a}^{*}$ is a cluster tilting object.

We call the above $\left(T / T_{a}\right) \oplus T_{a}^{*}$ the cluster tilting mutation of $T$ at a, and we denote it by $\mu_{a}(T)$. We have the following key observation [BMR, Theorem 6.1]. 
THEOREM 4.9. (1) We have a bijection

$$
\widetilde{X}:\left\{\text { cluster tilting objects in } \mathcal{C}_{Q}\right\} / \simeq \rightarrow\left\{\text { seeds in } \mathcal{A}_{Q}\right\}
$$

defined by

$$
T=\bigoplus_{a \in I} T_{a} \mapsto\left(Q_{T},\left\{X_{T_{a}}\right\}_{a \in I}\right)
$$

(2) We have $\tilde{X} \circ \mu_{a}=\mu_{a} \circ \tilde{X}$ for any $a \in I$.

We have

$$
\widetilde{X}_{P}=(Q, x(0))
$$

We number elements of $I_{+}$and $I_{-}$as $\left\{a_{1}, \ldots, a_{s}\right\}$ and $\left\{a_{s+1}, \ldots, a_{r}\right\}$, respectively. Define composed cluster tilting mutations by

$$
\mu_{+}:=\mu_{a_{s}} \cdots \mu_{a_{1}}, \quad \mu_{-}:=\mu_{a_{r}} \cdots \mu_{a_{s+1}}, \quad \mu:=\mu_{-} \mu_{+} .
$$

For a cluster tilting object $T=\bigoplus_{a \in I} T_{a}$, let $[T]_{a}$ denote $T_{a}$; similarly, for a seed $(R, y)$ of $\mathcal{A}_{Q}$, let $[(R, y)]_{a}$ denote $y_{a}$. By Theorem $4.9(2)$, we have the following relationship between the seed mutation and the cluster tilting mutation:

$$
\left[\mu^{k}\left(\widetilde{X}_{T}\right)\right]_{a}=X_{\left[\mu^{k}(T)\right]_{a}}, \quad\left[\mu_{+} \mu^{k}\left(\widetilde{X}_{T}\right)\right]_{a}=X_{\left[\mu_{+} \mu^{k}(T)\right]_{a}}
$$

for any cluster tilting object $T \in \mathcal{C}_{Q}, k \in \mathbb{Z}$, and $a \in I$. The following observation is a key result.

Proposition 4.10. For any $k \in \mathbb{Z}$ and $a \in I$, the following assertions hold.

(1) $\left[\mu^{k}(P)\right]_{a} \simeq \tau^{-k}\left(P_{a}\right)$ in $\mathcal{C}_{Q}$.

(2) $\left[\mu_{+} \mu^{k}(P)\right]_{a} \simeq \tau^{-k}\left(U_{a}\right)$ in $\mathcal{C}_{Q}$.

Proof. Since cluster tilting mutation commutes with any autoequivalence of $\mathcal{C}_{Q}$, we have only to show the assertion for $k=1$ for (1) and $k=0$ for (2).

(2) We put $I^{i}:=\left\{a_{1}, \ldots, a_{i}\right\}$ and

$$
T^{i}:=\left(\bigoplus_{a \in I^{i}} \tau^{-1}\left(P_{a}\right)\right) \oplus\left(\bigoplus_{a \in I \backslash I^{i}} P_{a}\right) .
$$


Then $T^{i}$ is a tilting $K Q$-module by [ASS]. Thus it is a cluster tilting object by [BMRRT]. Since $T^{i-1}$ and $T^{i}$ have the same indecomposable direct summands except $P_{a_{i}}$, we have $\mu_{a_{i}}\left(T^{i-1}\right)=T^{i}$. In particular, we have $\mu_{+}(P)=U$.

(1) By a similar argument to (2), we have $\mu(P)=\mu_{-}(U)=\tau^{-1}(P)$.

We use the following classical periodicity result.

Proposition 4.11. (1) We have that $\tau^{-h}(X)$ is isomorphic to $X[2]$ for any $X \in \mathcal{D}_{Q}$.

(2) We have that $\tau^{-h-2}(X)$ is isomorphic to $X$ for any $X \in \mathcal{C}_{Q}$.

Proof. (1) This follows from the structure of the Auslander-Reiten quiver of $\mathcal{D}_{Q}[\mathrm{G}]$, [Ha] (see Figure 3 ).

(2) By (1), we have $\tau^{-h-2}(X) \simeq \tau^{-2}(X[2]) \simeq F^{2}(X) \simeq X$.

Now we are ready to prove Theorem 4.4(2) (full-periodicity).
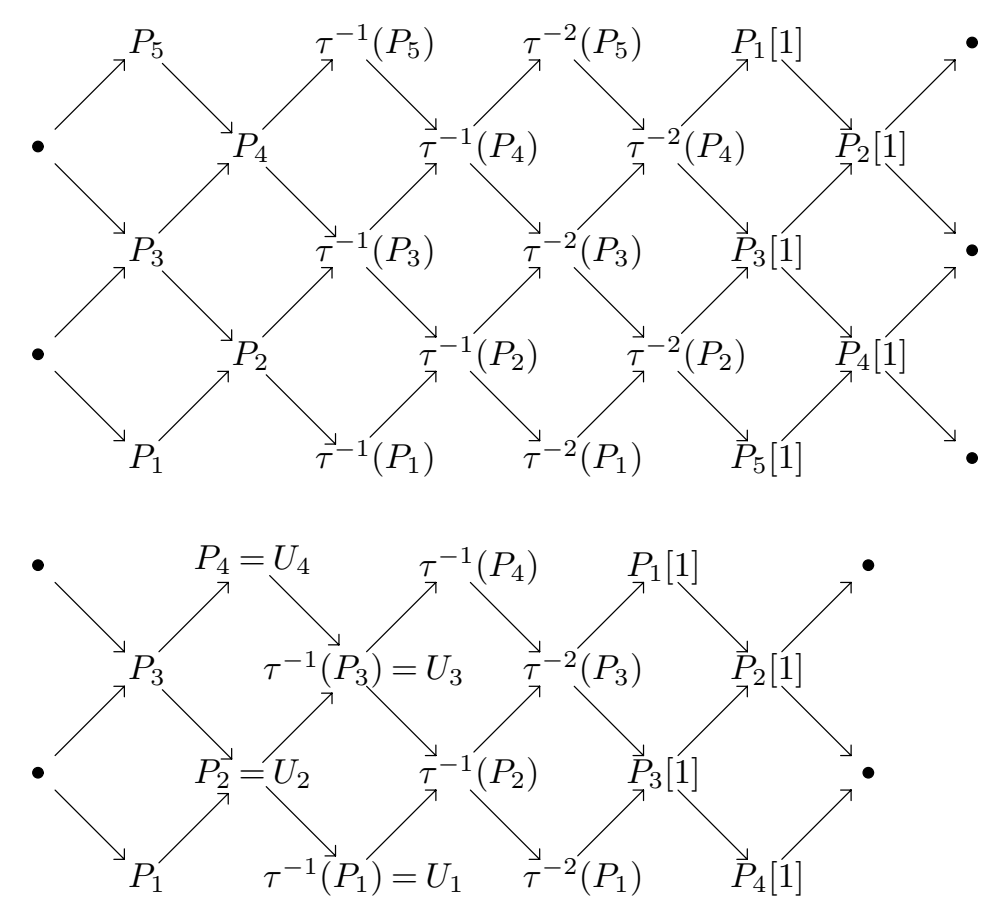

Figure 3: The Auslander-Reiten quivers of $\mathcal{D}_{Q}$ for $X_{r}=A_{5}$ (the above) and $A_{4}$ (the below) 
TheOREM 4.12 (Theorem 4.4(2)). $\left[\mu^{h+2}(Q, x(0))\right]_{a}=x_{a}(0)$.

Proof. We have $\left[\mu^{h+2}(P)\right]_{a} \stackrel{\text { Prop.4.10(1) }}{=} \tau^{-h-2}\left(P_{a}\right) \stackrel{\text { Prop.4.11(2) }}{=} P_{a}$ for any $a \in$ $I$. Applying $\widetilde{X}$, we have

$$
\left[\mu^{h+2}(Q, x(0))\right]_{a} \stackrel{(4.25)}{=}\left[\mu^{h+2}\left(\widetilde{X}_{P}\right)\right]_{a} \stackrel{(4.27)}{=} X_{\left[\mu^{h+2}(P)\right]_{a}}=X_{P_{a}}=x_{a}(0) .
$$

Next we prove Theorem 4.4(1) (half-periodicity). We divide the proof into two cases.

\section{Case 1: $h$ is even.}

In this case, the map $\omega: I \rightarrow I$ induces the quiver automorphism $\omega: Q \rightarrow$ $Q$. Thus $\omega$ induces an automorphism $\omega: K Q \rightarrow K Q$ of our $K$-algebra $K Q$, and we have an autoequivalence

$$
\omega: \mathcal{D}_{Q} \rightarrow \mathcal{D}_{Q}
$$

of categories. We have $\omega\left(P_{a}\right)=P_{\omega(a)}$ for any $a \in I$.

We have the following periodicity.

Proposition 4.13. (1) $\tau^{-h / 2}(X) \simeq \omega(X[1])$ for any $X \in \mathcal{D}_{Q}$;

(2) $\tau^{-(h+2) / 2}(X) \simeq \omega(X)$ for any $X \in \mathcal{C}_{Q}$.

Proof. (1) This follows from the structure of the Auslander-Reiten quiver of $\mathcal{D}_{Q}[\mathrm{G}],[\mathrm{Ha}]$ (see Figure 3 ).

(2) By (1), we have $\tau^{-(h+2) / 2}(X) \simeq \tau^{-1}(\omega(X[1])) \simeq F(\omega(X)) \simeq \omega(X)$.

We prove the first part of Theorem 4.4(1):

Theorem 4.14 (Theorem 4.4(1-i)). $\left[\mu^{(h+2) / 2}(Q, x(0))\right]_{a}=x_{\omega(a)}(0)$.

Proof. We have $\left[\mu^{(h+2) / 2}(P)\right]_{a} \stackrel{\text { Prop.4.10(1) }}{=} \tau^{-(h+2) / 2}\left(P_{a}\right) \stackrel{\text { Prop.4.13(2) }}{=} P_{\omega(a)}$. Applying $\tilde{X}$, we have $\left[\mu^{(h+2) / 2}(Q, x(0))\right]_{a} \stackrel{(4.25)}{=}\left[\mu^{(h+2) / 2}\left(\widetilde{X}_{P}\right)\right]_{a} \stackrel{(4.27)}{=}$ $X_{\left[\mu^{(h+2) / 2}(P)\right]_{a}}=X_{P_{\omega(a)}}=x_{\omega(a)}(0)$.

\section{Case 2: $h$ is odd.}

In this case, the map $\omega: I \rightarrow I$ induces the quiver isomorphism $\omega: Q \rightarrow$ $Q^{\text {op }}$. Thus $\omega$ induces an isomorphism $\omega: K Q \rightarrow K Q^{\text {op }}$ of $K$-algebras, and we have an equivalence

$$
\omega: \mathcal{D}_{Q} \rightarrow \mathcal{D}_{Q^{\mathrm{op}}}
$$


of categories. On the other hand, the tilting $K Q$-module $U$ in Example 4.7(2) induces an equivalence

$$
U \stackrel{\text { L }}{\otimes} Q_{Q^{\text {op }}}-: \mathcal{D}_{Q^{\text {op }}} \rightarrow \mathcal{D}_{Q}
$$

Composing them, we have an autoequivalence

$$
r: \mathcal{D}_{Q} \stackrel{\omega}{\longrightarrow} \mathcal{D}_{Q^{\mathrm{op}}} \stackrel{U^{\mathrm{L}}{ }_{K Q^{\mathrm{op}}}-}{\longrightarrow} \mathcal{D}_{Q}
$$

We have $r\left(P_{a}\right)=U_{\omega(a)}$ and $r\left(U_{a}\right)=\tau^{-1}\left(P_{\omega(a)}\right)$ for any $a \in I$ (see Figure 3); hence, $r^{2}(X) \simeq \tau^{-1}(X)$ for any $X \in \mathcal{D}_{Q}$.

We have the following periodicity.

Proposition 4.15. (1) $r \tau^{-(h-1) / 2}(X) \simeq X[1]$ for any $X \in \mathcal{D}_{Q}$;

(2) $\tau^{-(h+1) / 2}(X) \simeq r^{-1}(X)$ for any $X \in \mathcal{C}_{Q}$.

Proof. (1) This follows from the structure of the Auslander-Reiten quiver of $\mathcal{D}_{Q}[\mathrm{G}],[\mathrm{Ha}]$ (see Figure 3 ).

(2) By (1), we have $r \tau^{-(h+1) / 2}(X) \simeq \tau^{-1}(X[1]) \simeq X$, from which the claim follows.

We prove the second part of Theorem 4.4(1).

TheOREM 4.16 (Theorem 4.4(1-ii)). $\left[\mu_{+} \mu^{(h+1) / 2}(Q, x(0))\right]_{a}=x_{\omega(a)}(0)$.

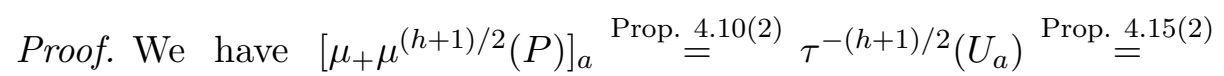
$r^{-1}\left(U_{a}\right)=P_{\omega(a)}$. Applying $\tilde{X}$, we have $\left[\mu_{+} \mu^{(h+1) / 2}(Q, x(0))\right]_{a} \stackrel{(4.25)}{=}$ $\left[\mu_{+} \mu^{(h+1) / 2}\left(\widetilde{X}_{P}\right)\right]_{a} \stackrel{(4.27)}{=} X_{\left[\mu_{+} \mu^{(h+1) / 2}(P)\right]_{a}}=X_{P_{\omega(a)}}=x_{\omega(a)}(0)$.

\subsection{Level greater than two case}

Here we study the periodicity of $\mathcal{T}_{\ell}\left(X_{r}\right)$ for simply laced $X_{r}$ and $\ell>2$. Since the case $X_{r}=A_{1}$ reduces to $\mathcal{T}_{2}\left(A_{\ell-1}\right)$ by the level-rank duality (Example 3.10), we continue to assume $X_{r} \neq A_{1}$.

First, let us establish a connection between the ring $\mathcal{T}_{\ell}^{\circ}\left(X_{r}\right)$ and the cluster algebra $\mathcal{A}_{Q \square Q^{\prime}}$ considered in [Kel2], [HL]. In doing that, we slightly generalize the problem and consider a pair of simply laced Dynkin diagrams $X_{r}, X_{r^{\prime}}^{\prime}\left(\neq A_{1}\right)$. For $X_{r}$ (resp. $\left.X_{r^{\prime}}^{\prime}\right)$, let $C, h, I, I_{ \pm}, \varepsilon, Q, \omega\left(\operatorname{resp} . C^{\prime}, h^{\prime}\right.$, $\left.I^{\prime}, I_{ \pm}^{\prime}, \varepsilon^{\prime}, Q^{\prime}, \omega^{\prime}\right)$ be the same as Section 4.2. 
Definition 4.17. The T-system $\mathbb{T}\left(X_{r}, X_{r^{\prime}}^{\prime}\right)$ of type $\left(X_{r}, X_{r^{\prime}}^{\prime}\right)$ is the following system of relations for a family of variables $T=\left\{T_{a, b}(u) \mid a \in I, b \in\right.$ $\left.I^{\prime}, u \in \mathbb{Z}\right\}$ :

$$
T_{a, b}(u-1) T_{a, b}(u+1)=\prod_{k \in I^{\prime}: C_{b k}^{\prime}=-1} T_{a, k}(u)+\prod_{k \in I: C_{a k}=-1} T_{k, b}(u) .
$$

Definition 4.18. The T-algebra $\mathcal{T}\left(X_{r}, X_{r^{\prime}}^{\prime}\right)$ of type $\left(X_{r}, X_{r^{\prime}}^{\prime}\right)$ is the ring with generators $T_{a, b}(u)^{ \pm 1}\left(a \in I, b \in I^{\prime}, u \in \mathbb{Z}\right)$ and the relations $\mathbb{T}\left(X_{r}, X_{r^{\prime}}^{\prime}\right)$. Also, we define the ring $\mathcal{T}^{\circ}\left(X_{r}, X_{r^{\prime}}^{\prime}\right)$ as the subring of $\mathcal{T}\left(X_{r}, X_{r^{\prime}}^{\prime}\right)$ generated by $T_{a, b}(u)\left(a \in I, b \in I^{\prime}, u \in \mathbb{Z}\right)$.

The system $\mathbb{T}\left(X_{r}, X_{r^{\prime}}^{\prime}\right)$ is the counterpart of the $Y$-system of [RTV] studied in [Kel2], and $\mathcal{T}_{\ell}\left(X_{r}\right)=\mathcal{T}\left(X_{r}, A_{\ell-1}\right)$ by identifying $T_{m}^{(a)}(u)$ with $T_{a, m}(u)$. We are going to show the following periodicity of $\mathcal{T}\left(X_{r}, X_{r^{\prime}}^{\prime}\right)$ :

$$
\begin{aligned}
& \text { half-periodicity: } \quad T_{a, b}\left(u+h+h^{\prime}\right)=T_{\omega(a), \omega^{\prime}(b)}(u), \\
& \text { periodicity: } \quad T_{a, b}\left(u+2\left(h+h^{\prime}\right)\right)=T_{a, b}(u) .
\end{aligned}
$$

Let $\mathcal{T}^{\circ}\left(X_{r}, X_{r^{\prime}}^{\prime}\right)_{ \pm}$be the subring of $\mathcal{T}^{\circ}\left(X_{r}, X_{r^{\prime}}^{\prime}\right)$ generated by $T_{a, b}(u)(a \in$ $\left.I, b \in I^{\prime}, u \in \mathbb{Z}\right)$ such that $\varepsilon(a) \varepsilon^{\prime}(b)(-1)^{u}= \pm$. Then, we have

$$
\begin{aligned}
\mathcal{T}^{\circ}\left(X_{r}, X_{r^{\prime}}^{\prime}\right) & \simeq \mathcal{T}^{\circ}\left(X_{r}, X_{r^{\prime}}^{\prime}\right)_{+} \otimes_{\mathbb{Z}} \mathcal{T}^{\circ}\left(X_{r}, X_{r^{\prime}}^{\prime}\right)_{-}, \\
\mathcal{T}^{\circ}\left(X_{r}, X_{r^{\prime}}^{\prime}\right)_{+} & \simeq \mathcal{T}^{\circ}\left(X_{r}, X_{r^{\prime}}^{\prime}\right)_{-} .
\end{aligned}
$$

To describe the corresponding cluster algebra to $\mathcal{T}^{\circ}\left(X_{r}, X_{r^{\prime}}^{\prime}\right)$, we introduce two kinds of quivers, $Q \square Q^{\prime}$ and $Q \otimes Q^{\prime}$.

Definition 4.19 ([Kel2]). (i) The square product $Q \square Q^{\prime}$ of $Q$ and $Q^{\prime}$ is the quiver obtained from the product $Q \times Q^{\prime}$ by reversing all the arrows in the full subquivers $\{a\} \times Q^{\prime}(a$ : sink of $Q)$ and $Q \times\{b\}\left(b\right.$ : source of $\left.Q^{\prime}\right)$.

(ii) The tensor product $Q \otimes Q^{\prime}$ of $Q$ and $Q^{\prime}$ is the quiver obtained from the product $Q \times Q^{\prime}$ by adding an arrow $\left(a_{2}, b_{2}\right) \rightarrow\left(a_{1}, b_{1}\right)$ for each pair of arrows $a_{1} \rightarrow a_{2}$ of $Q$ and $b_{1} \rightarrow b_{2}$ of $Q^{\prime}$.

EXAmPLE 4.20. Since $a \in I_{+}$is a source of $Q$, in our convention, the ordinary product $Q \times Q^{\prime}$ consists of the following type of squares:

$$
Q \times Q^{\prime}: \begin{array}{ccc}
(+-) & \rightarrow & (--) \\
\uparrow & & \uparrow \\
(++) & \rightarrow & (-+)
\end{array}
$$


where $(+-)$, for example, represents a vertex $(a, b)$ of $Q \times Q^{\prime}$ with $a \in I_{+}$, $b \in I_{-}^{\prime}$. Correspondingly, $Q \square Q^{\prime}$ and $Q \otimes Q^{\prime}$ consist of the following types of squares:

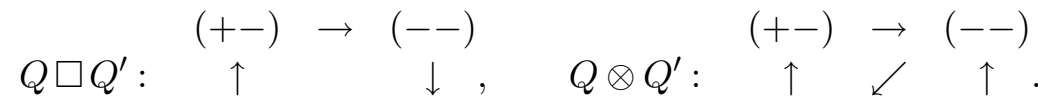

$$
\begin{aligned}
& (++) \leftarrow(-+) \quad(++) \rightarrow(-+)
\end{aligned}
$$

Using these diagrams, one can easily check that

$$
\begin{aligned}
Q \square Q^{\prime} & =Q^{\mathrm{op}} \square Q^{\mathrm{op}}, \\
\left(Q \square Q^{\prime}\right)^{\mathrm{op}} & =Q^{\mathrm{op}} \square Q^{\prime}=Q \square Q^{\mathrm{op}}, \\
\left(Q \otimes Q^{\prime}\right)^{\mathrm{op}} & =Q^{\mathrm{op}} \otimes Q^{\mathrm{op}} .
\end{aligned}
$$

We define composed mutations,

$$
\mu_{ \pm \pm}=\prod_{(a, b) \in I_{ \pm} \times I_{ \pm}^{\prime}} \mu_{a, b}, \quad \mu_{ \pm \mp}=\prod_{(a, b) \in I_{ \pm} \times I_{\mp}^{\prime}} \mu_{a, b},
$$

where $\mu_{a, b}$ is the mutation at $(a, b)$. Then, the following cycle of mutations of quivers occurs (the "eyeglass diagram"):

$$
\begin{aligned}
& Q \otimes Q^{\prime \text { op }} \quad Q \otimes Q^{\prime} \\
& \mu_{++} \nearrow \nwarrow \mu_{--} \mu_{+-} \nearrow \quad \searrow \mu_{-+} \\
& Q \square Q^{\prime} \quad\left(Q \square Q^{\prime}\right)^{\mathrm{op}} \quad Q \square Q^{\prime} \text {. } \\
& \mu_{--} \searrow \quad \nearrow^{\mu_{++}} \mu_{-+} \searrow \quad \nearrow^{\mu_{+-}} \\
& Q^{\mathrm{op}} \otimes Q^{\prime} \quad Q^{\mathrm{op}} \otimes Q^{\mathrm{op}}
\end{aligned}
$$

We further define composed mutations [Kel2]

$$
\begin{aligned}
& \mu_{-}=\mu_{+-} \mu_{--}, \quad \mu_{+}=\mu_{++} \mu_{-+}, \\
& \mu_{\otimes}=\mu_{-} \mu_{+}=\mu_{+-} \mu_{--} \mu_{++} \mu_{-+} .
\end{aligned}
$$

In particular, $\mu_{\otimes}$ preserves $Q \otimes Q^{\prime}$.

REMARK 4.21. (1) The mutations $\mu_{ \pm \pm}, \mu_{ \pm \mp}$, and $\mu_{\otimes}$ here correspond to $\mu_{\mp, \mp}, \mu_{\mp, \pm}$, and the inverse of $\mu_{\otimes}$ in [Kel2]. This is due to our convention of the assignment of $+/-$ for the sources/sinks of $Q$ and $Q^{\prime}$ and is not essential at all.

(2) Instead of (4.40), one may set $\mu_{+}=\mu_{+-} \mu_{++}, \mu_{-}=\mu_{--} \mu_{-+}$, and $\mu_{\otimes}=\mu_{+} \mu_{-}$. This is again a matter of choice. 
We introduce the $\left(I \times I^{\prime}\right)$-tuple of variables $x=\left\{x_{a, b} \mid a \in I, b \in I^{\prime}\right\}$, and we define $\mathcal{A}_{Q \square Q^{\prime}}$ to be the cluster algebras with initial seeds $\left(Q \square Q^{\prime}, x\right)$. We set $x=x(0)$, and we define clusters $x(u), z(u), \bar{z}(u)(u \in \mathbb{Z})$ of $\mathcal{A}_{Q \square Q^{\prime}}$ by the following sequence of mutations ( $u$ : even):

$$
\begin{array}{cccc}
\left(Q \otimes Q^{\mathrm{op}}, z(u+1)\right) & \left(Q \otimes Q^{\prime}, z(u+2)\right) \\
\mu_{++} \nearrow & \nwarrow \mu_{--} & \mu_{+-} \nearrow & \nwarrow \mu_{-+} \\
\left(Q \square Q^{\prime}, x(u)\right) & \left(\left(Q \square Q^{\prime}\right)^{\mathrm{op}}, x(u+1)\right) & \left(Q \square Q^{\prime}, x(u+2)\right) . \\
\mu_{--} \searrow & \nearrow^{\mu_{++}} & \mu_{-+} \searrow & \nearrow^{\mu_{+-}} \\
\left(Q^{\mathrm{op}} \otimes Q^{\prime}, \bar{z}(u+1)\right) & \left(Q^{\mathrm{op}} \otimes Q^{\mathrm{op}}, \bar{z}(u+2)\right)
\end{array}
$$

In particular,

$$
\begin{aligned}
& x_{a, b}(u+1)=x_{a, b}(u) \text { if } \varepsilon(a) \varepsilon^{\prime}(b)(-1)^{u}=-, \\
& z_{a, b}(u)= \begin{cases}x_{a, b}(u) & a \in I_{+}, \\
x_{a, b}(u-1) & a \in I_{-},\end{cases} \\
& \bar{z}_{a, b}(u)= \begin{cases}x_{a, b}(u-1) & a \in I_{+}, \\
x_{a, b}(u) & a \in I_{-},\end{cases} \\
& \left(Q \otimes Q^{\prime}, z(2 k)\right)=\mu_{\otimes}^{k}\left(Q \otimes Q^{\prime}, z(0)\right) \quad(k \in \mathbb{Z}), \\
& \left(Q \otimes Q^{\prime \text { op }}, z(2 k+1)\right)=\mu_{+} \mu_{\otimes}^{k}\left(Q \otimes Q^{\prime}, z(0)\right) \quad(k \in \mathbb{Z}) .
\end{aligned}
$$

Lemma 4.22. The family $\left\{x_{a, b}(u) \mid a \in I, b \in I^{\prime}, u \in \mathbb{Z}\right\}$ satisfies the $T$ system $\mathbb{T}\left(X_{r}, X_{r^{\prime}}^{\prime}\right)$ in $\mathcal{A}_{Q \square Q^{\prime}} ;$ namely,

$$
x_{a, b}(u-1) x_{a, b}(u+1)=\prod_{k \in I^{\prime}: C_{b k}^{\prime}=-1} x_{a, k}(u)+\prod_{k \in I: C_{a k}=-1} x_{k, b}(u) .
$$

Proof. The proof is the same as Lemma 4.1 by replacing $\mu_{+}$(resp. $\mu_{-}$) therein with $\mu_{--} \mu_{++}$(resp. $\mu_{-+} \mu_{+-}$).

Define a ring homomorphism $f: \mathcal{A}_{Q \square Q^{\prime}} \rightarrow \mathcal{T}\left(X_{r}, X_{r^{\prime}}^{\prime}\right)$ as the restriction of the ring homomorphism $\mathbb{Z}\left[x_{a, b}^{ \pm 1}\right]_{(a, b) \in I \times I^{\prime}} \rightarrow \mathcal{T}\left(X_{r}, X_{r^{\prime}}^{\prime}\right)$ given by

$$
f: x_{a, b}^{ \pm 1} \mapsto \begin{cases}T_{a, b}(0)^{ \pm 1} & \varepsilon(a) \varepsilon^{\prime}(b)=+ \\ T_{a, b}(1)^{ \pm 1} & \varepsilon(a) \varepsilon^{\prime}(b)=-.\end{cases}
$$

Then, as Lemma 4.2, we have the following. 
Lemma 4.23. For the above homomorphism $f: \mathcal{A}_{Q \square Q^{\prime}} \rightarrow \mathcal{T}\left(X_{r}, X_{r^{\prime}}^{\prime}\right)$,

$$
f: x_{a, b}(u) \mapsto \begin{cases}T_{a, b}(u) & \varepsilon(a) \varepsilon^{\prime}(b)(-1)^{u}=+, \\ T_{a, b}(u+1) & \varepsilon(a) \varepsilon^{\prime}(b)(-1)^{u}=-.\end{cases}
$$

Let $\mu_{\square}:=\mu_{-+} \mu_{+-} \mu_{--} \mu_{++}$. We define $\mathcal{A}_{Q \square Q^{\prime}}^{\mathrm{t}}$ to be the subring of $\mathcal{A}_{Q \square Q^{\prime}}$ generated by $x_{a, b}(u)\left(a \in I, b \in I^{\prime}, u \in 2 \mathbb{Z}\right)$, that is, the cluster variables belonging to the seeds $\mu_{\square}^{k}\left(Q \square Q^{\prime}, x(0)\right)(k \in \mathbb{Z})$. We call $\mathcal{A}_{Q \square Q^{\prime}}^{\mathrm{t}}$ the translation subalgebra of $\mathcal{A}_{Q \square Q^{\prime}}$ with respect to $\mu_{\square}$. The ring $\mathcal{A}_{Q \square Q^{\prime}}^{\mathrm{t}}{ }^{\prime}$ is no longer a cluster algebra. We remark that, by (4.42), the ring $\mathcal{A}_{Q \square Q^{\prime}}^{\mathrm{t}}$ coincides with the subring of $\mathcal{A}_{Q \square Q^{\prime}}$ generated by $x_{a, b}(u)\left(a \in I, b \in I^{\prime}, u \in \mathbb{Z}\right)$, which are the cluster variables belonging to the "bipartite belt" in [FZ4, Section 8].

Proposition 4.24. The ring $\mathcal{T}^{\circ}\left(X_{r}, X_{r^{\prime}}^{\prime}\right)$ is isomorphic to $\mathcal{A}_{Q \square Q^{\prime}}^{\mathrm{t}} \otimes_{\mathbb{Z}}$ $\mathcal{A}_{Q \square Q^{\prime}}^{\mathrm{t}}$

Proof. By Lemma 4.23, the restriction $f: \mathcal{A}_{Q \square Q^{\prime}}^{\mathrm{t}} \rightarrow \mathcal{T}^{\circ}\left(X_{r}, X_{r^{\prime}}^{\prime}\right)_{+}$is surjective. Furthermore, the inverse correspondence $g: \mathcal{T}^{\circ}\left(X_{r}, X_{r^{\prime}}^{\prime}\right)_{+} \rightarrow \mathcal{A}_{Q \square Q^{\prime}}^{\mathrm{t}}$, $T_{a, b}(u) \mapsto x_{a, b}(u)$ defines a homomorphism by Lemma 4.22 and the fact that $\mathcal{A}_{Q \square Q^{\prime}}^{\mathrm{t}}$ is an integral domain. Therefore,

$$
\mathcal{T}^{\circ}\left(X_{r}, X_{r^{\prime}}^{\prime}\right)_{+} \simeq \mathcal{A}_{Q \square Q^{\prime}}^{\mathrm{t}}
$$

and we obtain the assertion.

REMARK 4.25. Hernandez and Leclerc [HL] also study the relation between the cluster algebra $\mathcal{A}_{Q \square Q^{\prime}}$ with $X_{r^{\prime}}^{\prime}=A_{r^{\prime}}$ and the T-system in view of the categorification of $\mathcal{A}_{Q} \square Q^{\prime}$ by a subcategory of the category of the finite-dimensional $U_{q}(\hat{\mathfrak{g}})$-modules.

Now let us turn to the periodicity problem. Let $\mathcal{A}_{Q \otimes Q^{\prime}}$ be the cluster algebra with initial seed $\left(Q \otimes Q^{\prime}, z\right), z=\left\{z_{a, b} \mid a \in I, b \in I^{\prime}\right\}$. Two cluster algebras $\mathcal{A}_{Q \otimes Q^{\prime}}$ and $\mathcal{A}_{Q \square Q^{\prime}}$ coincide by setting $z=z(0)$ in (4.41). A crucial observation made by Keller [Kel2] is that the periodicity of $\mathcal{A}_{Q \square Q^{\prime}}$ is more transparent in the " $\otimes$-picture" than in the " $\square$-picture" from the cluster categorical point of view. By (4.43) and (4.47), it is easy to check that the periodicity (4.33) of $\mathcal{T}\left(X_{r}, X_{r^{\prime}}^{\prime}\right)$ is translated as

$$
\begin{aligned}
& \text { half-periodicity: } \quad z_{a, b}\left(u+h+h^{\prime}\right)= \begin{cases}z_{\omega(a), \omega^{\prime}(b)}(u) & h \text { : even, } \\
\bar{z}_{\omega(a), \omega^{\prime}(b)}(u) & h \text { : odd, }\end{cases} \\
& \text { periodicity: } \quad z_{a, b}\left(u+2\left(h+h^{\prime}\right)\right)=z_{a, b}(u) .
\end{aligned}
$$


The following periodicity of $\mathcal{A}_{Q \otimes Q^{\prime}}$ is immediately obtained from the results in [Kel2].

TheOREM 4.26 (Keller [Kel2]). The following equality holds for $\mathcal{A}_{Q \otimes Q^{\prime}}$ (u: even):

$$
\mu_{\otimes}^{h+h^{\prime}}\left(Q \otimes Q^{\prime}, z(u)\right) \stackrel{\mathrm{id}}{=}\left(Q \otimes Q^{\prime}, z(u)\right) .
$$

Proof. The $F$-polynomials of $\mathcal{A}_{Q \otimes Q^{\prime}}$ relevant to the above periodicity are expressed in terms of the triangulated category $\mathcal{C}_{K Q \otimes K Q^{\prime}}[\mathrm{Kel} 2$, Theorem 7.13(c)]; furthermore, $\mathcal{C}_{K Q \otimes K Q^{\prime}}$ has the desired periodicity [Kel2, Proposition 8.5]. Therefore, the cluster variable $z(u)$ has the same periodicity by [FZ4, Corollary 6.3, Proposition 6.9].

As a refinement of Theorem 4.26, we also show the half-periodicity of $\mathcal{A}_{Q \otimes Q^{\prime}}$

THEOREM 4.27. The following equalities hold for $\mathcal{A}_{Q \otimes Q^{\prime}}$ (u: even).

(1) For $\left(h, h^{\prime}\right)=($ even, even $)$,

$$
\mu_{\otimes}^{\left(h+h^{\prime}\right) / 2}\left(Q \otimes Q^{\prime}, z(u)\right) \stackrel{\omega \times \omega^{\prime}}{=}\left(Q \otimes Q^{\prime}, z(u)\right) .
$$

(2) For $\left(h, h^{\prime}\right)=($ odd, odd $)$,

$$
\mu_{\otimes}^{\left(h+h^{\prime}\right) / 2}\left(Q \otimes Q^{\prime}, z(u)\right) \stackrel{\omega \times \omega^{\prime}}{=}\left(Q^{\mathrm{op}} \otimes Q^{\prime \mathrm{op}}, \bar{z}(u)\right) .
$$

(3) For $\left(h, h^{\prime}\right)=($ even, odd $)$,

$$
\mu_{+} \mu_{\otimes}^{\left(h+h^{\prime}-1\right) / 2}\left(Q \otimes Q^{\prime}, z(u)\right) \stackrel{\omega \times \omega^{\prime}}{=}\left(Q \otimes Q^{\prime}, z(u)\right) .
$$

(4) For $\left(h, h^{\prime}\right)=($ odd, even $)$,

$$
\mu_{+} \mu_{\otimes}^{\left(h+h^{\prime}-1\right) / 2}\left(Q \otimes Q^{\prime}, z(u)\right) \stackrel{\omega \times \omega^{\prime}}{=}\left(Q^{\mathrm{op}} \otimes Q^{\prime \mathrm{op}}, \bar{z}(u)\right) .
$$

The proof of Theorem 4.27 is given in Section 4.5.

COROLlary 4.28. The following relations hold in $\mathcal{T}\left(X_{r}, X_{r^{\prime}}^{\prime}\right)$ :

(1) Half-periodicity: $T_{a, b}\left(u+h+h^{\prime}\right)=T_{\omega(a), \omega^{\prime}(b)}(u)$.

(2) Periodicity: $T_{a, b}\left(u+2\left(h+h^{\prime}\right)\right)=T_{a, b}(u)$.

Proof. The relations in (4.49) immediately follow from Theorems 4.26 and 4.27 and (4.44). 
By Corollaries 4.5 and 4.28, we obtain the main result of this section, which is as follows.

COROLlary 4.29. The following relations hold in $\mathcal{T}_{\ell}\left(X_{r}\right)$ for any simply laced $X_{r}$ and any $\ell \geq 2$.

(1) Half-periodicity: $T_{m}^{(a)}(u+h+\ell)=T_{\ell-m}^{(\omega(a))}(u)$.

(2) Periodicity: $T_{m}^{(a)}(u+2(h+\ell))=T_{m}^{(a)}(u)$.

\subsection{Proof of Theorems 4.26 and 4.27 by cluster category}

Here we prove Theorem 4.27 by adapting the method of [Kel2, Theorem 8.2] for our situation. In the course we also include a proof of Theorem 4.26 without using the $F$-polynomials, for the reader's convenience. We present the proof as parallel as possible to the level 2 case in Section 4.3.

Let $Q$ and $Q^{\prime}$ continue to be the alternating quivers in Section 4.4 whose underlying graphs are simply laced $X_{r}$ and $X_{r^{\prime}}^{\prime}$ other than $A_{1}$, respectively. For $Q$ (resp. $\left.Q^{\prime}\right)$, let $K Q, \mathcal{D}_{Q}, \tau: \mathcal{D}_{Q} \rightarrow \mathcal{D}_{Q}\left(\operatorname{resp} . K Q^{\prime}, \mathcal{D}_{Q^{\prime}}, \tau^{\prime}: \mathcal{D}_{Q^{\prime}} \rightarrow\right.$ $\left.\mathcal{D}_{Q^{\prime}}\right)$ be the ones in Section 4.3.

We denote the tensor product $\otimes_{K}$ simply by $\otimes$, and we define a finite dimensional $K$-algebra $A$ by

$$
A:=K Q \otimes K Q^{\prime}
$$

Let $\mathcal{D}_{A}=\mathcal{D}^{\mathrm{b}}(\bmod A)$ be the bounded derived category of finite dimensional $A$-modules, and let

$$
\tau_{\otimes}:=D A[-1] \stackrel{\mathbf{L}}{\otimes}{ }_{A}-: \mathcal{D}_{A} \rightarrow \mathcal{D}_{A}
$$

be the Auslander-Reiten translation for $\mathcal{D}_{A}$. Now we define another autoequivalence of $\mathcal{D}_{A}$ by $F:=\tau_{\otimes}^{-1} \circ[1]$. We need the following easy observation.

Lemma 4.30. We have $\tau(X) \otimes \tau^{\prime}(Y) \simeq F^{-1}(X \otimes Y)$ in $\mathcal{D}_{A}$ for any $X \in$ $\mathcal{D}_{Q}$ and $Y \in \mathcal{D}_{Q^{\prime}}$.

Proof. We have $\tau(X) \otimes \tau^{\prime}(Y) \simeq\left(D(K Q) \stackrel{\mathbf{L}}{\otimes_{K Q}} X[-1]\right) \otimes\left(D\left(K Q^{\prime}\right) \stackrel{\mathbf{L}}{\otimes_{K Q^{\prime}}}\right.$ $Y[-1]) \simeq\left(D(K Q) \otimes D\left(K Q^{\prime}\right)\right)[-2] \stackrel{\mathbf{L}}{\otimes_{A}}(X \otimes Y)=F^{-1}(X \otimes Y)$.

The orbit category

$$
\mathcal{D}_{A} / F
$$


has the same objects with $\mathcal{D}_{A}$, and the morphism space is given by

$$
\operatorname{Hom}_{\left(\mathcal{D}_{A} / F\right)}(X, Y):=\bigoplus_{i \in \mathbf{Z}} \operatorname{Hom}_{\mathcal{D}_{A}}\left(X, F^{i}(Y)\right)
$$

for any $X, Y \in \mathcal{D}_{A} / F$. In contrast to (4.17), $\mathcal{D}_{A} / F$ is no longer a triangulated category in general. However, based on the works of Keller (see [Kel1], [Kel4]), Amiot [A, Section 4] constructed a triangulated hull $\mathcal{C}_{A}$ of $\mathcal{D}_{A} / F$, which is a 2-Calabi-Yau triangulated category with a fully faithful functor $\mathcal{D}_{A} / F \rightarrow \mathcal{C}_{A}$ satisfying a certain universal property. (Here we need the fact that the functor $H^{0}(F-)$ is nilpotent on $\bmod A$, which follows from Lemma 4.30.) We call $\mathcal{C}_{A}$ the (generalized) cluster category of $A$.

We say that an object $T=\bigoplus_{(a, b) \in I \times I^{\prime}} T_{a, b} \in \mathcal{C}_{A}$ is cluster tilting if

(1) each $T_{a, b}$ is indecomposable and mutually nonisomorphic, and

(2) $\operatorname{add} T=\left\{X \in \mathcal{C}_{A} \mid \operatorname{Hom}_{\mathcal{C}_{A}}(T, X[1])=0\right\}$.

(To simplify our proof, we assume that the index set of direct summands of $T$ is $I \times I^{\prime}$. This does not affect the definition essentially due to Example 4.31 and [DeK, Theorem 2.4].) For a cluster tilting object $T \in \mathcal{C}_{A}$, we denote by $Q_{T}$ the quiver of the endomorphism ring $\operatorname{End}_{\mathcal{C}_{A}}(T)$ [ARS], [ASS].

ExAmple 4.31 (cf. Example 4.7). For $a \in I$ and $b \in I^{\prime}$, we denote by $e_{a}$ and $e_{b}^{\prime}$ the paths of length zero in $Q$ and $Q^{\prime}$, respectively. As in Example 4.7, we define $K Q$-modules $P, U$ and $K Q^{\prime}$-modules $P^{\prime}, U^{\prime}$ by

$$
\begin{aligned}
& P_{a}:=(K Q) e_{a}, \quad P:=K Q=\bigoplus_{a \in I} P_{a}, \\
& U_{a}:=\left\{\begin{array}{ll}
\tau^{-1}\left(P_{a}\right) & a \in I_{+}, \\
P_{a} & a \in I_{-},
\end{array} \quad U:=\bigoplus_{a \in I} U_{a},\right. \\
& P_{b}^{\prime}:=\left(K Q^{\prime}\right) e_{b}^{\prime},
\end{aligned}
$$

Then

$$
P \otimes P^{\prime}=\bigoplus_{(a, b) \in I \times I^{\prime}} P_{a} \otimes P_{b}^{\prime}
$$

is a cluster tilting object in $\mathcal{C}_{A}$ with $Q_{P \otimes P^{\prime}}=Q \otimes Q^{\prime}$ [A, Theorem 4.10]. 
Again we can define the mutation of cluster tilting objects as follows [IY, Theorem 5.3].

Theorem 4.32 (cf. Theorem 4.8). Let $T=\bigoplus_{(a, b) \in I \times I^{\prime}} T_{a, b} \in \mathcal{C}_{A}$ be a cluster tilting object. For any $(a, b) \in I \times I^{\prime}$, there exists a unique indecomposable object $T_{a, b}^{*} \in \mathcal{C}_{A}$ that is not isomorphic to $T_{a, b}$ such that $\left(T / T_{a, b}\right) \oplus T_{a, b}^{*}$ is a cluster tilting object.

We call the above $\left(T / T_{a, b}\right) \oplus T_{a, b}^{*}$ the cluster tilting mutation of $T$ at $(a, b)$, and we denote it by $\mu_{a, b}(T)$.

We number elements of $I_{-} \times I_{+}^{\prime}$ as $\left\{c_{1}, \ldots, c_{s}\right\}\left(s:=\left|I_{-} \times I_{+}^{\prime}\right|\right)$, and we define a composed cluster tilting mutation $\mu_{-+}$by

$$
\mu_{-+}:=\mu_{c_{s}} \cdots \mu_{c_{1}} \text {. }
$$

Similarly, we define

$$
\mu_{++}, \quad \mu_{--}, \quad \mu_{+-}
$$

by using $I_{+} \times I_{+}^{\prime}, I_{-} \times I_{-}^{\prime}$, and $I_{+} \times I_{-}^{\prime}$, respectively. We further define

$$
\mu_{\otimes}:=\mu_{+-} \mu_{--} \mu_{++} \mu_{-+}, \quad \mu_{+}:=\mu_{++} \mu_{-+} .
$$

Thus we have a numbering $\left\{c_{1}, \ldots, c_{r r^{\prime}}\right\}$ of the elements of $I \times I^{\prime}$ such that $\mu_{\otimes}=\mu_{c_{r r^{\prime}}} \cdots \mu_{c_{1}}$.

For a cluster tilting object $T=\bigoplus_{(a, b) \in I \times I^{\prime}} T_{a, b}$, let $[T]_{a, b}$ denote $T_{a, b}$. We have the following key observation.

Proposition 4.33 (cf. Proposition 4.10). (1) We have a diagram

$$
\begin{aligned}
& P \otimes \tau^{\prime-k}\left(P^{\prime}\right) \quad P \otimes \tau^{\prime-k}\left(U^{\prime}\right) \quad P \otimes \tau^{\prime-k-1}\left(P^{\prime}\right) \\
& \searrow \mu_{-+} \mu_{++} \nearrow \quad \searrow \mu_{--} \mu_{+-} \nearrow \\
& V^{k} W^{k}
\end{aligned}
$$

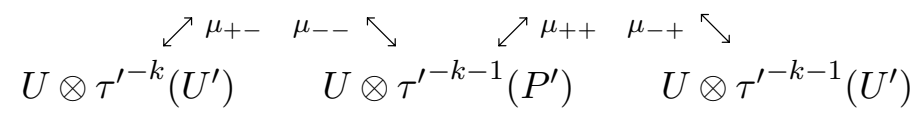

of composed cluster tilting mutations for any $k \in \mathbb{Z}$, where

$\begin{aligned} \text { (4.68) } \quad V^{k} & :=\left(\bigoplus_{(a, b) \in I_{-} \times I_{+}^{\prime}} P_{a} \otimes \tau^{\prime-k-1}\left(P_{b}^{\prime}\right)\right) \oplus\left(\bigoplus_{(a, b) \notin I_{-} \times I_{+}^{\prime}} P_{a} \otimes \tau^{\prime-k}\left(P_{b}^{\prime}\right)\right), \\ \text { (4.69) } \quad W^{k}: & =\left(\bigoplus_{(a, b) \notin I_{+} \times I_{-}^{\prime}} P_{a} \otimes \tau^{\prime-k-1}\left(P_{b}^{\prime}\right)\right) \oplus\left(\bigoplus_{(a, b) \in I_{+} \times I_{-}^{\prime}} P_{a} \otimes \tau^{\prime-k}\left(P_{b}^{\prime}\right)\right) .\end{aligned}$ 
(2) We have

$$
\begin{array}{r}
{\left[\mu_{\otimes}^{k}\left(P \otimes P^{\prime}\right)\right]_{a, b}=P_{a} \otimes \tau^{\prime-k}\left(P_{b}^{\prime}\right),} \\
{\left[\mu_{+} \mu_{\otimes}^{k}\left(P \otimes P^{\prime}\right)\right]_{a, b}=P_{a} \otimes \tau^{\prime-k}\left(U_{b}^{\prime}\right)}
\end{array}
$$

for any $k \in \mathbb{Z}$ and $(a, b) \in I \times I^{\prime}$.

Proof. (2) is an immediate consequence of (1).

(1) We show only $\mu_{-+}\left(P \otimes P^{\prime}\right)=V^{0}$ since other cases are shown similarly. We put $J^{\ell}:=\left\{c_{i} \mid 1 \leq i \leq \ell\right\}$. Define $T^{\ell} \in \mathcal{D}_{A}$ by

$$
T^{\ell}:=\left(\bigoplus_{(a, b) \in J^{\ell}} P_{a} \otimes \tau^{\prime-1}\left(P_{b}^{\prime}\right)\right) \oplus\left(\bigoplus_{(a, b) \in\left(I \times I^{\prime}\right) \backslash J^{\ell}} P_{a} \otimes P_{b}^{\prime}\right)
$$

for any $0 \leq \ell \leq s=\left|I_{-} \times I_{+}^{\prime}\right|$. Clearly we have $T^{0}=P \otimes P^{\prime}$ and $T^{s}=V^{0}$.

The following observation is crucial.

Proposition 4.34. (1) $T^{\ell}$ is a tilting A-module for any $0 \leq \ell \leq s$.

(2) The algebra $B:=\operatorname{End}_{A}\left(T^{\ell}\right)$ has global dimension at most two.

(3) For any simple $B$-modules $S$ and $S^{\prime}$, we have $\operatorname{Ext}_{B}^{1}(S, S)=0$, and either $\operatorname{Ext}_{B}^{1}\left(S, S^{\prime}\right)=0$ or $\operatorname{Ext}_{B}^{2}\left(S, S^{\prime}\right)=0$ holds.

Proof. (1) Since $Q^{\prime}$ is not of type $A_{1}$, our $T^{\ell}$ is an $A$-module. Clearly $T^{\ell}$ has projective dimension at most one, and the number of indecomposable direct summands of $T^{\ell}$ is $r r^{\prime}$. Thus we have only to show $\operatorname{Ext}_{A}^{1}\left(T^{\ell}, T^{\ell}\right)=0$. Fix $(a, b),(c, d) \in I \times I^{\prime}$, and we shall show

$$
\operatorname{Hom}_{\mathcal{D}_{A}}\left(T_{a, b}^{\ell}, T_{c, d}^{\ell}[1]\right)=0 .
$$

We have a general equality

$$
\begin{aligned}
\operatorname{Hom}_{\mathcal{D}_{A}}\left(X \otimes X^{\prime},\left(Y \otimes Y^{\prime}\right)[i]\right) \\
\quad=\bigoplus_{j+j^{\prime}=i} \operatorname{Hom}_{\mathcal{D}_{Q}}(X, Y[j]) \otimes \operatorname{Hom}_{\mathcal{D}_{Q^{\prime}}}\left(X^{\prime}, Y^{\prime}\left[j^{\prime}\right]\right)
\end{aligned}
$$

for any $X, Y \in \mathcal{D}_{Q}$ and $X^{\prime}, Y^{\prime} \in \mathcal{D}_{Q^{\prime}}$. We also have

$$
\operatorname{Hom}_{\mathcal{D}_{Q}}\left(P_{a}, P_{c}[j]\right)=0
$$

if " $j \neq 0$ " or " $j=0, a \neq c$, and there is no arrow $a \rightarrow c$ in $Q . "$

We divide the proof of (4.71) into four cases. 
(i) Assume $(a, b),(c, d) \notin J^{\ell}$. Then (4.71) follows from (4.72), (4.73), and

$$
\operatorname{Hom}_{\mathcal{D}_{Q^{\prime}}}\left(P_{b}^{\prime}, P_{d}^{\prime}\left[j^{\prime}\right]\right)=0 \text { if } j^{\prime} \neq 0 \text {. }
$$

(ii) Assume $(a, b),(c, d) \in J^{\ell}$. Then (4.71) follows from (4.72), (4.73), and

$$
\begin{aligned}
& \operatorname{Hom}_{\mathcal{D}_{Q^{\prime}}}\left(\tau^{\prime-1}\left(P_{b}^{\prime}\right), \tau^{\prime-1}\left(P_{d}^{\prime}\right)\left[j^{\prime}\right]\right) \\
& \simeq \operatorname{Hom}_{\mathcal{D}_{Q^{\prime}}}\left(P_{b}^{\prime}, P_{d}^{\prime}\left[j^{\prime}\right]\right)=0 \text { if } j^{\prime} \neq 0 .
\end{aligned}
$$

(iii) Assume $(a, b) \in J^{\ell}$ and $(c, d) \notin J^{\ell}$. We have

$$
\begin{aligned}
& \operatorname{Hom}_{\mathcal{D}_{Q^{\prime}}}\left(\tau^{\prime-1}\left(P_{b}^{\prime}\right), P_{d}^{\prime}\left[j^{\prime}\right]\right) \simeq D \operatorname{Hom}_{\mathcal{D}_{Q^{\prime}}}\left(P_{d}^{\prime}\left[j^{\prime}\right], P_{b}^{\prime}[1]\right)=0 \\
& \quad \text { if " } j^{\prime} \neq 1 \text { " or " } j^{\prime}=1, b \neq d, \text { and there is no arrow } d \rightarrow b \text { in } Q^{\prime} \text {." }
\end{aligned}
$$

Since $a \in I_{-}, b \in I_{+}^{\prime}$, and $(a, b) \neq(c, d)$ hold, either " $a \neq c$ and there is no arrow $a \rightarrow c$ in $Q$ " or " $b \neq d$ and there is no arrow $d \rightarrow b$ in $Q^{\prime}$." Thus (4.71) follows from (4.72) and (4.73).

(iv) Assume $(a, b) \notin J^{\ell}$ and $(c, d) \in J^{\ell}$. Since $d \in I_{+}^{\prime}$ and $Q^{\prime}$ is not type $A_{1}$, we have

$$
\operatorname{Hom}_{\mathcal{D}_{Q^{\prime}}}\left(P_{b}^{\prime}, \tau^{\prime-1}\left(P_{d}^{\prime}\right)\left[j^{\prime}\right]\right)=0 \quad \text { if } j^{\prime} \neq 0
$$

Thus (4.71) follows from (4.72) and (4.73).

(2) and (3) It is enough to prove the following claim.

Claim. Every simple B-module $S$ has a projective resolution

$$
0 \rightarrow B_{2} \rightarrow B_{1} \rightarrow B_{0} \rightarrow S \rightarrow 0
$$

such that $B_{1}$ and $B_{0} \oplus B_{2}$ have no nonzero common direct summand.

Let $(a, b) \in I \times I^{\prime}$ be such that $S$ is the top of the indecomposable projective $B$-module $\operatorname{Hom}_{A}\left(T^{\ell}, T_{a, b}^{\ell}\right)$. We divide the proof of the claim into four cases.

(i) Assume that $a \in I_{+}$. Then the radical of the $A$-module $P_{a} \otimes P_{b}^{\prime}$ is $\bigoplus_{d \rightarrow b} P_{a} \otimes P_{d}^{\prime}$, which belongs to add $T^{\ell}$. Here the direct sum is taken over all arrows in $Q^{\prime}$ with target $b$. Thus we have a projective resolution

$$
0 \rightarrow \operatorname{Hom}_{A}\left(T^{\ell}, \bigoplus_{d \rightarrow b} P_{a} \otimes P_{d}^{\prime}\right) \rightarrow \operatorname{Hom}_{A}\left(T^{\ell}, P_{a} \otimes P_{b}^{\prime}\right) \rightarrow S \rightarrow 0
$$


and the claim follows.

(ii) Assume that $(a, b) \in\left(I_{-} \times I_{+}^{\prime}\right) \backslash J^{\ell}$. Then the radical of the $A$-module $P_{a} \otimes P_{b}^{\prime}$ is $\bigoplus_{c \rightarrow a} P_{c} \otimes P_{b}^{\prime}$, which belongs to add $T^{\ell}$. Thus we have a projective resolution

$$
0 \rightarrow \operatorname{Hom}_{A}\left(T^{\ell}, \bigoplus_{c \rightarrow a} P_{c} \otimes P_{b}^{\prime}\right) \rightarrow \operatorname{Hom}_{A}\left(T^{\ell}, P_{a} \otimes P_{b}^{\prime}\right) \rightarrow S \rightarrow 0
$$

and the claim follows.

(iii) Assume that $(a, b) \in J^{\ell}$. Applying $\left(P_{a} \otimes-\right)$ to the Auslander-Reiten sequence $0 \rightarrow P_{b}^{\prime} \rightarrow \bigoplus_{b \rightarrow d} P_{d}^{\prime} \rightarrow \tau^{\prime-1}\left(P_{b}^{\prime}\right) \rightarrow 0$ of $K Q^{\prime}$-modules, we have an exact sequence

$$
0 \rightarrow P_{a} \otimes P_{b}^{\prime} \rightarrow \bigoplus_{b \rightarrow d} P_{a} \otimes P_{d}^{\prime} \stackrel{f}{\rightarrow} P_{a} \otimes \tau^{\prime-1}\left(P_{b}^{\prime}\right) \rightarrow 0
$$

of $A$-modules whose middle term belongs to add $T^{\ell}$. Clearly, any morphism $T^{\ell} \rightarrow P_{a} \otimes \tau^{\prime-1}\left(P_{b}^{\prime}\right)$ that is not a split epimorphism factors through $f$. Moreover, the left term $P_{a} \otimes P_{b}^{\prime}$ does not belong to add $T^{\ell}$, but its radical $\bigoplus_{c \rightarrow a} P_{c} \otimes P_{b}^{\prime}$ belongs to add $T^{\ell}$. Consequently, we have a projective resolution

$$
\begin{gathered}
0 \rightarrow \operatorname{Hom}_{A}\left(T^{\ell}, \bigoplus_{c \rightarrow a} P_{c} \otimes P_{b}^{\prime}\right) \rightarrow \operatorname{Hom}_{A}\left(T^{\ell}, \bigoplus_{b \rightarrow d} P_{a} \otimes P_{d}^{\prime}\right) \\
\stackrel{f}{\rightarrow} \operatorname{Hom}_{A}\left(T^{\ell}, P_{a} \otimes \tau^{\prime-1}\left(P_{b}^{\prime}\right)\right) \rightarrow S \rightarrow 0
\end{gathered}
$$

and the claim follows.

(iv) Assume $(a, b) \in I_{-} \times I_{-}^{\prime}$. Taking a tensor product of exact sequences $0 \rightarrow \bigoplus_{c \rightarrow a} P_{c} \rightarrow P_{a}$ and $0 \rightarrow \bigoplus_{d \rightarrow b,(a, d) \notin J^{\ell}} P_{d}^{\prime} \rightarrow P_{b}^{\prime}$, we have an exact sequence

$$
\begin{aligned}
0 \rightarrow & \bigoplus \begin{array}{c}
c \rightarrow a \\
d \rightarrow b \\
(a, d) \notin J^{\ell}
\end{array} \\
& \rightarrow\left(\bigoplus_{\substack{d \rightarrow b \\
(a, d) \notin J^{\ell}}} P_{a} \otimes P_{d}^{\prime}\right.
\end{aligned}
$$

of $A$-modules whose terms belong to add $T^{\ell}$. Clearly, any morphism $T^{\ell} \rightarrow$ $P_{a} \otimes P_{b}^{\prime}$ which is not a split epimorphism factors through $f$. Thus we have 
a projective resolution

$$
\begin{gathered}
0 \rightarrow \operatorname{Hom}_{A}\left(T^{\ell}, \bigoplus_{\substack{c \rightarrow a \\
d \rightarrow b \\
(a, d) \notin J^{\ell}}} P_{c} \otimes P_{d}^{\prime}\right) \\
\rightarrow \operatorname{Hom}_{A}\left(T^{\ell},\left(\bigoplus_{\substack{d \rightarrow b \\
(a, d) \notin J^{\ell}}} P_{a} \otimes P_{d}^{\prime}\right) \oplus\left(\bigoplus_{c \rightarrow a} P_{c} \otimes P_{b}^{\prime}\right)\right) \\
\stackrel{f}{\rightarrow} \operatorname{Hom}_{A}\left(T^{\ell}, P_{a} \otimes P_{b}^{\prime}\right) \rightarrow S \rightarrow 0
\end{gathered}
$$

and the claim follows.

By Proposition 4.34(1)-(2) together with [A, Theorem 4.10], $T^{\ell}$ is a cluster tilting object in $\mathcal{C}_{A}$ for any $1 \leq \ell \leq s$. Since $T^{\ell-1}$ and $T^{\ell}$ have the same indecomposable direct summands except $T_{c_{\ell}}^{\ell}$, we have $\mu_{c_{\ell}}\left(T^{\ell-1}\right)=T^{\ell}$. Consequently, we have $\mu_{-+}\left(P \otimes P^{\prime}\right)=\mu_{-+}\left(T^{0}\right)=T^{s}=V^{0}$.

We define a set $\mathbf{T}$ of cluster tilting objects by

$$
\mathbf{T}=\left\{\mu_{c_{i}} \cdots \mu_{c_{1}} \mu_{\otimes}^{u}\left(P \otimes P^{\prime}\right) \mid u \in \mathbf{Z}, 0 \leq i<r r^{\prime}\right\} .
$$

We have the following result by [Kel2, Proposition 8.3].

Proposition 4.35. The quiver $Q_{T}$ has no loops and 2-cycles for any $T \in \mathbf{T}$.

Proof. This is a consequence of Proposition 4.34(3) and [A, Proposition 4.16].

The following result is crucial in our proof.

TheOREM 4.36 (cf. Theorems 4.6 and 4.9). There exists a map

$$
X:\{\text { indecomposable direct summands of objects in } \mathbf{T}\} / \simeq
$$

$$
\rightarrow\left\{\text { cluster variables in } \mathcal{A}_{Q \otimes Q^{\prime}}\right\}
$$

such that we have a map

$$
\widetilde{X}: \mathbf{T} \rightarrow\left\{\text { seeds in } \mathcal{A}_{Q \otimes Q^{\prime}}\right\}
$$

defined by

$$
T=\bigoplus_{(a, b) \in I \times I^{\prime}} T_{a, b} \mapsto\left(Q_{T},\left\{X_{T_{a, b}}\right\}_{(a, b) \in I \times I^{\prime}}\right)
$$

satisfying the following conditions. 
(1) $\tilde{X}_{P \otimes P^{\prime}}=\left(Q \otimes Q^{\prime}, z(0)\right)$.

(2) If $T$ and $\mu_{a, b}(T)$ belong to $\mathbf{T}$, then $\widetilde{X}_{\mu_{a, b}(T)}=\mu_{a, b}\left(\widetilde{X}_{T}\right)$.

Proof. The assertion follows from Proposition 4.35 [Pal, Theorem 4] and [BIRS, Theorem I.1.6].

By Theorem 4.36, (4.41), and (4.67), we also obtain

$$
\begin{aligned}
& \widetilde{X}_{U \otimes U^{\prime}}=\left(Q^{\mathrm{op}} \otimes Q^{\mathrm{op}}, \bar{z}(0)\right), \\
& \widetilde{X}_{P \otimes U^{\prime}}=\left(Q \otimes Q^{\mathrm{op}}, z(1)\right), \quad \widetilde{X}_{U \otimes P^{\prime}}=\left(Q^{\mathrm{op}} \otimes Q^{\prime}, \bar{z}(-1)\right) .
\end{aligned}
$$

Now we are ready to prove Theorem 4.26 (full-periodicity).

Proposition 4.37. We use the following periodicity result by [Kel2, Proposition 8.5]: $X \otimes \tau^{\prime-h-h^{\prime}}(Y) \simeq X \otimes Y$ in $\mathcal{C}_{A}$ for any $X \in \mathcal{D}_{Q}$ and $Y \in \mathcal{D}_{Q^{\prime}}$.

Proof. We have $X \otimes \tau^{\prime-h-h^{\prime}}(Y) \simeq F^{-h}\left(X \otimes{\tau^{\prime}-h-h^{\prime}}^{\prime}(Y)\right) \stackrel{\text { Lem.4.30 }}{\simeq} \tau^{h}(X) \otimes$ $\tau^{\prime-h^{\prime}}(Y) \stackrel{\text { Prop.4.11(1) }}{\simeq} X[-2] \otimes Y[2] \simeq X \otimes Y$.

For a seed $(R, y)$ of $\mathcal{A}_{Q \otimes Q^{\prime}}$, let $[(R, y)]_{a, b}$ denote $y_{a, b}$.

TheOREM 4.38 (Theorem 4.26). Now we are ready to prove Theorem 4.26 (full-periodicity): $\left[\mu_{\otimes}^{h+h^{\prime}}\left(Q \otimes Q^{\prime}, z(0)\right)\right]_{a, b}=z_{a, b}(0)$.

Proof. We have $\left[\mu_{\otimes}^{h+h^{\prime}}\left(P \otimes P^{\prime}\right)\right]_{a, b} \stackrel{\text { Prop.4.33(2) }}{=} P_{a} \otimes \tau^{\prime-h-h^{\prime}}\left(P_{b}^{\prime}\right) \stackrel{\text { Prop.4.37 }}{=}$ $P_{a} \otimes P_{b}^{\prime}$ for any $(a, b) \in I \times I^{\prime}$. Applying $\widetilde{X}$, we have $\left[\mu_{\otimes}^{h+h^{\prime}}\left(Q \otimes Q^{\prime}\right.\right.$, $z(0))]_{a, b} \stackrel{\text { Thm.4.36(1) }}{=}\left[\mu_{\otimes}^{h+h^{\prime}}\left(\tilde{X}_{P \otimes P^{\prime}}\right)\right]_{a, b} \stackrel{\text { Thm.4.36(2) }}{=} \quad X_{\left[\mu_{\otimes}^{h+h^{\prime}}\left(P \otimes P^{\prime}\right)\right]_{a, b}}=$ $X_{P_{a} \otimes P_{b}^{\prime}}=z_{a, b}(0)$.

Next we prove Theorem 4.27 (half-periodicity). We divide the proof into four cases.

Recall the following facts in Section 4.3: when $h$ is even, the map $\omega: I \rightarrow I$ induces the quiver automorphism $\omega: Q \rightarrow Q$, the $K$-algebra automorphism $\omega: K Q \rightarrow K Q$, and an autoequivalence $\omega: \mathcal{D}_{Q} \rightarrow \mathcal{D}_{Q}$. We have $\omega\left(P_{a}\right)=$ $P_{\omega(a)}$ for any $a \in I$. When $h$ is odd, the map $\omega: I \rightarrow I$ induces the quiver isomorphism $\omega: Q \rightarrow Q^{\mathrm{op}}$, the $K$-algebra isomorphism $\omega: K Q \rightarrow K Q^{\mathrm{op}}$, and an autoequivalence

$$
r: \mathcal{D}_{Q} \stackrel{\omega}{\rightarrow} \mathcal{D}_{Q^{\mathrm{op}}} \stackrel{U^{\mathrm{L}} \otimes_{K Q^{\mathrm{op}}-}}{\longrightarrow} \mathcal{D}_{Q} .
$$


We have $r\left(P_{a}\right)=U_{\omega(a)}$ for any $a \in I$, and we have $r^{2}(X) \simeq \tau^{-1}(X)$ for any $X \in \mathcal{D}_{Q}$.

We also define $\omega^{\prime}: \mathcal{D}_{Q^{\prime}} \rightarrow \mathcal{D}_{Q^{\prime}}$ for even $h^{\prime}$, and we define $r^{\prime}: \mathcal{D}_{Q^{\prime}} \rightarrow \mathcal{D}_{Q^{\prime}}$ for odd $h^{\prime}$ in the same way.

Case 1: Both $h$ and $h^{\prime}$ are even.

Proposition 4.39. We have $X \otimes \tau^{\prime-\left(h+h^{\prime}\right) / 2}(Y) \simeq \omega(X) \otimes \omega^{\prime}(Y)$ in $\mathcal{C}_{A}$ for any $X \in \mathcal{D}_{Q}$ and $Y \in \mathcal{D}_{Q^{\prime}}$.

Proof. We have $X \otimes \tau^{\prime-\left(h+h^{\prime}\right) / 2}(Y) \simeq F^{-h / 2}\left(X \otimes \tau^{\prime-\left(h+h^{\prime}\right) / 2}(Y)\right) \stackrel{\text { Lem.4.30 }}{\simeq}$ $\tau^{h / 2}(X) \otimes \tau^{\prime-h^{\prime} / 2}(Y) \stackrel{\text { Prop.4.13(1) }}{\simeq} \omega^{-1}(X[-1]) \otimes \omega^{\prime}(Y[1]) \simeq \omega(X) \otimes \omega^{\prime}(Y)$.

THEOREM 4.40 (Theorem 4.27(1)). We have the following:

$$
\left[\mu_{\otimes}^{\left(h+h^{\prime}\right) / 2}\left(Q \otimes Q^{\prime}, z(0)\right)\right]_{a, b}=z_{\omega(a), \omega^{\prime}(b)}(0) .
$$

Proof. We have

$$
\left[\mu_{\otimes}^{\left(h+h^{\prime}\right) / 2}\left(P \otimes P^{\prime}\right)\right]_{a, b} \stackrel{\text { Prop.4.33(2) }}{=} P_{a} \otimes \tau^{\prime-\left(h+h^{\prime}\right) / 2}\left(P_{b}^{\prime}\right) \stackrel{\text { Prop.4.39 }}{=} P_{\omega(a)} \otimes P_{\omega^{\prime}(b)}^{\prime} \text {. }
$$

Applying $\tilde{X}$, we have

$$
\begin{aligned}
{\left[\mu_{\otimes}^{\left(h+h^{\prime}\right) / 2}\left(Q \otimes Q^{\prime}, z(0)\right)\right]_{a, b} } & \stackrel{\text { Thm. }}{=}{ }^{\text {Thm. }} \stackrel{.36(1)}{=}\left[\mu_{\otimes}^{\left(h+h^{\prime}\right) / 2}\left(\widetilde{X}_{P \otimes P^{\prime}}\right)\right]_{a, b} \\
& =X_{P_{\omega(a)} \otimes \mu_{\omega^{\prime}(b)}^{\left(h+h^{\prime}\right) / 2}}=z_{\omega(a), \omega^{\prime}(b)}(0) .
\end{aligned}
$$

Case 2: Both $h$ and $h^{\prime}$ are odd.

Proposition 4.41. We have $X \otimes \tau^{\prime-\left(h+h^{\prime}\right) / 2}(Y) \simeq r(X) \otimes r^{\prime}(Y)$ in $\mathcal{C}_{A}$ for any $X \in \mathcal{D}_{Q}$ and $Y \in \mathcal{D}_{Q^{\prime}}$.

Proof. We have

$$
\begin{aligned}
& X \otimes \tau^{\prime-\left(h+h^{\prime}\right) / 2}(Y) \simeq F^{-(h-1) / 2}\left(X \otimes \tau^{\prime-\left(h+h^{\prime}\right) / 2}(Y)\right) \\
& \stackrel{\text { Lem.4.30 }}{\simeq} \tau^{(h-1) / 2}(X) \otimes \tau^{\prime-1} \tau^{\prime-\left(h^{\prime}-1\right) / 2}(Y) \\
& \stackrel{\text { Prop.4.15(1) }}{\simeq} r(X[-1]) \otimes \tau^{\prime-1} r^{\prime-1}(Y[1]) \simeq r(X) \otimes r^{\prime}(Y) .
\end{aligned}
$$


TheOREM 4.42 (Theorem 4.27(2)). We have

$$
\left[\mu_{\otimes}^{\left(h+h^{\prime}\right) / 2}\left(Q \otimes Q^{\prime}, z(0)\right)\right]_{a, b}=\bar{z}_{\omega(a), \omega^{\prime}(b)}(0) .
$$

Proof. We have

$$
\begin{gathered}
{\left[\mu_{\otimes}^{\left(h+h^{\prime}\right) / 2}\left(P \otimes P^{\prime}\right)\right]_{a, b} \stackrel{\text { Prop.4.33(2) }}{=} P_{a} \otimes \tau^{\prime-\left(h+h^{\prime}\right) / 2}\left(P_{b}^{\prime}\right)} \\
\stackrel{\text { Prop.4.41 }}{=} r\left(P_{a}\right) \otimes r^{\prime}\left(P_{b}^{\prime}\right)=U_{\omega(a)} \otimes U_{\omega^{\prime}(b)}^{\prime}
\end{gathered}
$$

Applying $\tilde{X}$, we have

$$
\begin{aligned}
& {\left[\mu_{\otimes}^{\left(h+h^{\prime}\right) / 2}\left(Q \otimes Q^{\prime}, z(0)\right)\right]_{a, b} } \stackrel{\text { Thm.4.36(1) }}{=}\left[\mu_{\otimes}^{\left(h+h^{\prime}\right) / 2}\left(\widetilde{X}_{P \otimes P^{\prime}}\right)\right]_{a, b} \\
& \stackrel{\text { Thm.4.36(2) }}{=} X_{\left[\mu_{\otimes}^{\left(h+h^{\prime}\right) / 2}\left(P \otimes P^{\prime}\right)\right]_{a, b}} \\
&=X_{U_{\omega(a)} \otimes U_{\omega^{\prime}(b)}^{\prime}}=\bar{z}_{\omega(a), \omega^{\prime}(b)}(0) .
\end{aligned}
$$

Case 3: $h$ is even, and $h^{\prime}$ is odd.

Proposition 4.43. We have $X \otimes \tau^{\prime-\left(h+h^{\prime}-1\right) / 2}(Y) \simeq \omega(X) \otimes r^{\prime-1}(Y)$ in $\mathcal{C}_{A}$ for any $X \in \mathcal{D}_{Q}$ and $Y \in \mathcal{D}_{Q^{\prime}}$.

Proof. We have

$$
\begin{aligned}
& X \otimes \tau^{\prime-\left(h+h^{\prime}-1\right) / 2}(Y) \simeq F^{-h / 2}\left(X \otimes \tau^{\prime-\left(h+h^{\prime}-1\right) / 2}(Y)\right) \\
& \stackrel{\text { Lem.4.30 }}{\simeq} \tau^{h / 2}(X) \otimes \tau^{\prime-\left(h^{\prime}-1\right) / 2}(Y) \\
& \text { Prop.4.13(1),4.15(1) } \omega^{-1}(X[-1]) \otimes r^{\prime-1}(Y[1]) \\
& \simeq \omega(X) \otimes r^{\prime-1}(Y) .
\end{aligned}
$$

TheOREM 4.44 (Theorem 4.27(3)). We have $\left[\mu_{+} \mu_{\otimes}^{\left(h+h^{\prime}-1\right) / 2}\left(Q \otimes Q^{\prime}\right.\right.$, $z(0))]_{a, b}=z_{\omega(a), \omega^{\prime}(b)}(0)$.

Proof. We have

$$
\left[\mu_{+} \mu_{\otimes}^{\left(h+h^{\prime}-1\right) / 2}\left(P \otimes P^{\prime}\right)\right]_{a, b} \stackrel{\text { Prop.4.33(2) }}{=} P_{a} \otimes \tau^{\prime-\left(h+h^{\prime}-1\right) / 2}\left(U_{b}^{\prime}\right)
$$




$$
\begin{aligned}
& \stackrel{\text { Prop.4.43 }}{=} P_{\omega(a)} \otimes r^{\prime-1}\left(U_{b}^{\prime}\right) \\
& \quad=P_{\omega(a)} \otimes P_{\omega^{\prime}(b)}^{\prime} .
\end{aligned}
$$

Applying $\tilde{X}$, we have

$$
\begin{aligned}
& {\left[\mu_{+} \mu_{\otimes}^{\left(h+h^{\prime}-1\right) / 2}\left(Q \otimes Q^{\prime}, z(0)\right)\right]_{a, b} } \stackrel{\text { Thm.4.36(1) }}{=}\left[\mu_{+} \mu_{\otimes}^{\left(h+h^{\prime}-1\right) / 2}\left(\widetilde{X}_{P \otimes P^{\prime}}\right)\right]_{a, b} \\
& \stackrel{\text { Thm.4.36(2) }}{=} X_{\left[\mu_{+} \mu_{\otimes}^{\left(h+h^{\prime}-1\right) / 2}\left(P \otimes P^{\prime}\right)\right]_{a, b}} \\
&=X_{P_{\omega(a)} \otimes P_{\omega^{\prime}(b)}^{\prime}}=z_{\omega(a), \omega^{\prime}(b)}(0) .
\end{aligned}
$$

Case 4: $h$ is odd, and $h^{\prime}$ is even.

Proposition 4.45. We have $X \otimes \tau^{\prime-\left(h+h^{\prime}-1\right) / 2}(Y) \simeq r(X) \otimes \omega^{\prime}(Y)$ in $\mathcal{C}_{A}$ for any $X \in \mathcal{D}_{Q}$ and $Y \in \mathcal{D}_{Q^{\prime}}$.

Proof. We have

$$
\begin{aligned}
& X \otimes \tau^{\prime-\left(h+h^{\prime}-1\right) / 2}(Y) \simeq F^{-(h-1) / 2}\left(X \otimes \tau^{-\left(h+h^{\prime}-1\right) / 2}(Y)\right) \\
& \stackrel{\text { Lem.4.30 }}{\simeq} \tau^{(h-1) / 2}(X) \otimes \tau^{\prime-\left(h^{\prime} / 2\right)}(Y)
\end{aligned}
$$$$
\text { Prop.4.13(1),4.15(1) } r(X[-1]) \otimes \omega^{\prime}(Y[1]) \simeq r(X) \otimes \omega^{\prime}(Y) \text {. }
$$

THEOREM 4.46 (Theorem 4.27(4)). We have $\left[\mu_{+} \mu_{\otimes}^{\left(h+h^{\prime}-1\right) / 2}\left(Q \otimes Q^{\prime}\right.\right.$, $z(0))]_{a, b}=\bar{z}_{\omega(a), \omega^{\prime}(b)}(0)$.

Proof. We have

$$
\begin{aligned}
{\left[\mu_{+} \mu_{\otimes}^{\left(h+h^{\prime}-1\right) / 2}\left(P \otimes P^{\prime}\right)\right]_{a, b} \stackrel{\text { Prop.4.33(2) }}{=} P_{a} \otimes \tau^{\prime-\left(h+h^{\prime}-1\right) / 2}\left(U_{b}^{\prime}\right) } \\
\stackrel{\text { Prop.4.45 }}{=} r\left(P_{a}\right) \otimes \omega\left(U_{b}^{\prime}\right)=U_{\omega(a)} \otimes U_{\omega^{\prime}(b)}^{\prime} .
\end{aligned}
$$

Applying $\tilde{X}$, we have

$$
\begin{aligned}
& {\left[\mu_{+} \mu_{\otimes}^{\left(h+h^{\prime}-1\right) / 2}\left(Q \otimes Q^{\prime}, z(0)\right)\right]_{a, b} } \stackrel{\text { Thm.4.36(1) }}{=}\left[\mu_{+} \mu_{\otimes}^{\left(h+h^{\prime}-1\right) / 2}\left(\widetilde{X}_{P \otimes P^{\prime}}\right)\right]_{a, b} \\
& \stackrel{\text { Thm.4.36(2) }}{=} X_{\left[\mu_{+} \mu_{\otimes}^{\left(h+h^{\prime}-1\right) / 2}\left(P \otimes P^{\prime}\right)\right]_{a, b}} \\
& \quad=X_{U_{\omega(a)} \otimes U_{\omega^{\prime}(b)}^{\prime}}=\bar{z}_{\omega(a), \omega^{\prime}(b)}(0) .
\end{aligned}
$$




\section{§5. Determinant method I: $\mathcal{T}_{\ell}\left(A_{r}\right)$}

Volkov [V] proved the periodicity of the regular solutions of $\mathbb{Y}_{\ell}\left(A_{r}\right)$ in $\mathbb{C}$ for any $\ell \geq 2$ by constructing the manifestly periodic determinant expression. In the process of the proof, he essentially proved the periodicity of the regular solutions of $\mathbb{T}_{\ell}\left(A_{r}\right)$ in $\mathbb{C}$ as well. In this section, we prove the periodicities of $\mathcal{T}_{\ell}\left(A_{r}\right)$ and $y_{\ell}\left(A_{r}\right)$ for any $\ell \geq 2$ by reformulating the determinant method in our setting to avoid the projective geometrical arguments used in [V], with the application to the $C_{r}$ case also in mind.

\subsection{Level $\ell$ restricted T-system with spiral boundary condition}

Following $[\mathrm{V}]$, we introduce the level $\ell$ restricted T-system of type $A_{r}$ with a more general boundary condition than the unit boundary condition for $\mathbb{T}_{\ell}\left(A_{r}\right)$ in Definition 3.1; we call it the spiral boundary condition.

Let us set

$$
\begin{aligned}
H & =\{(a, m, u) \mid a=0, \ldots, r+1 ; m=0, \ldots, \ell ; u \in \mathbb{Z}\}, \\
H^{\circ} & =\{(a, m, u) \in H \mid a \neq 0, r+1 ; m \neq 0, \ell\}, \\
\partial H & =H \backslash H^{\circ}, \\
H_{e} & =\{(a, m, u) \in H \mid a+m+u \text { is even }\}, \\
H_{o} & =\{(a, m, u) \in H \mid a+m+u \text { is odd }\} .
\end{aligned}
$$

We also use the combined notations $H_{o}^{\circ}=H^{\circ} \cap H_{o}, \partial H_{e}=\partial H \cap H_{e}$, and so forth.

Definition 5.1. Fix an integer $\ell \geq 2$. The level $\ell$ restricted $T$-system $\widetilde{\mathbb{T}}_{\ell}\left(A_{r}\right)$ of type $A_{r}$ with the spiral boundary condition is the following system of relations for a family of variables $T=\left\{T_{m}^{(a)}(u) \mid(a, m, u) \in H\right\}$, as follows.

(1) T-system:

$$
\begin{array}{r}
T_{m}^{(a)}(u-1) T_{m}^{(a)}(u+1)=T_{m-1}^{(a)}(u) T_{m+1}^{(a)}(u)+T_{m}^{(a-1)}(u) T_{m}^{(a+1)}(u) \\
\left((a, m, u) \in H^{\circ}\right),
\end{array}
$$


(2) Spiral boundary condition:

$$
\begin{aligned}
T_{0}^{(a)}(u+1) & =T_{0}^{(a-1)}(u) & (a=1, \ldots, r+1), \\
T_{\ell}^{(a)}(u+1) & =T_{\ell}^{(a+1)}(u) & (a=0, \ldots, r), \\
T_{m}^{(0)}(u+1) & =T_{m+1}^{(0)}(u) & (m=0, \ldots, \ell-1), \\
T_{m}^{(r+1)}(u+1) & =T_{m-1}^{(r+1)}(u) & (m=1, \ldots, \ell) .
\end{aligned}
$$

DEFinition 5.2. Let $\widetilde{\mathcal{T}}_{\ell}\left(A_{r}\right)$ be the ring with generators $T_{m}^{(a)}(u)^{ \pm 1}((a, m$, $u) \in H)$ and the relations $\widetilde{\mathbb{T}}_{\ell}\left(A_{r}\right)$.

Condition (5.3) means that $T_{m}^{(a)}(u)$ is constant along spirals on the boundary $\partial H$. In particular, if we impose $T_{m}^{(a)}(u)=1$ for any $(a, m, u) \in \partial H$, then $\widetilde{\mathbb{T}}_{\ell}\left(A_{r}\right)$ reduces to $\mathbb{T}_{\ell}\left(A_{r}\right)$. In other words, $\mathcal{T}_{\ell}\left(A_{r}\right)$ is isomorphic to $\widetilde{\mathcal{T}}_{\ell}\left(A_{r}\right) / J$, where $J$ is the ideal of $\widetilde{\mathcal{T}}_{\ell}\left(A_{r}\right)$ generated by $T_{m}^{(a)}(u)-1((a, m, u) \in \partial H)$.

Recall that the dual Coxeter number of $A_{r}$ is $r+1$. We will prove the following.

THEOREM 5.3 (Henriques [Hen, Theorem 5]). The following relations hold in $\widetilde{\mathcal{T}}_{\ell}\left(A_{r}\right)$.

(1) Half-periodicity: $T_{m}^{(a)}(u+r+1+\ell)=T_{\ell-m}^{(r+1-a)}(u)$.

(2) Periodicity: $T_{m}^{(a)}(u+2(r+1+\ell))=T_{m}^{(a)}(u)$.

By the paragraph that follows Definition 5.2, we obtain the following.

Corollary 5.4. The following relations hold in $\mathcal{T}_{\ell}\left(A_{r}\right)$.

(1) Half-periodicity: $T_{m}^{(a)}(u+r+1+\ell)=T_{\ell-m}^{(r+1-a)}(u)$.

(2) Periodicity: $T_{m}^{(a)}(u+2(r+1+\ell))=T_{m}^{(a)}(u)$.

REMARK 5.5. Theorem 5.3(1) is a corollary of a more general theorem by Henriques [Hen, Theorem 5], since the spiral boundary condition ensures that $c=1$ for [Hen, (11)]. However, as we mentioned earlier, we prove Theorem 5.3 by the determinant method of [V] to have the application to the $C_{r}$ case in mind.

\subsection{Proof of Theorem $\mathbf{5 . 3}$}

Let $R$ be any ring. Let us take an arbitrary $\ell \times \infty$ matrix $M$ over $R$ such that $M=\left[x_{k}\right]_{k \in \mathbb{Z}}, x_{k} \in R^{\ell}$ with the following periodicity:

$$
x_{k+r+1+\ell}=(-1)^{\ell-1} x_{k} .
$$


Let $D_{M}=\left\{D_{m}^{(a)}(u) \mid(a, m, u) \in H_{e}\right\}$ be a family of minors of $M$ defined by

$$
\begin{aligned}
& D_{m}^{(a)}(u)=\operatorname{det}[\overbrace{x_{\beta} x_{\beta+1} \ldots}^{m} \overbrace{}^{v_{\ldots}} \vee \overbrace{\ldots}^{\ell-m} \vee \overbrace{\ldots x_{\beta+r+\ell}}^{\ell+1-a}], \\
& \beta=-\frac{a+m+u}{2} \in \mathbb{Z},
\end{aligned}
$$

where $\stackrel{\vee}{x_{k}}$ means the omission of $x_{k}$ as usual.

Proposition 5.6. The family $D_{M}=\left\{D_{m}^{(a)}(u) \mid(a, m, u) \in H_{e}\right\}$ satisfies the following relations in the ring $R$.

(1) T-system:

$$
\begin{array}{r}
D_{m}^{(a)}(u-1) D_{m}^{(a)}(u+1)=D_{m-1}^{(a)}(u) D_{m+1}^{(a)}(u)+D_{m}^{(a-1)}(u) D_{m}^{(a+1)}(u) \\
\left((a, m, u) \in H_{o}^{\circ}\right),
\end{array}
$$

(2) Spiral boundary condition:

$$
\begin{aligned}
D_{0}^{(a)}(u+1) & =D_{0}^{(a-1)}(u) & & (a=1, \ldots, r+1), \\
D_{\ell}^{(a)}(u+1) & =D_{\ell}^{(a+1)}(u) & & (a=0, \ldots, r), \\
D_{m}^{(0)}(u+1) & =D_{m+1}^{(0)}(u) & & (m=0, \ldots, \ell-1), \\
D_{m}^{(r+1)}(u+1) & =D_{m-1}^{(r+1)}(u) & & (m=1, \ldots, \ell) .
\end{aligned}
$$

(3) Half-periodicity:

$$
D_{m}^{(a)}(u+r+1+\ell)=D_{\ell-m}^{(r+1-a)}(u) .
$$

Proof. (1) They are the Plücker relations among minors.

(2) The first three relations follow from the definition of $D_{m}^{(a)}(u)$. To show the last relation, we also use the (anti-)periodicity (5.4) of $x_{k}$.

(3) It also follows from the definition of $D_{m}^{(a)}(u)$ and (5.4).

REMARK 5.7. This determinant solution of the T-system (5.7) is regarded as the restricted version of [KLWZ, (2.25)].

Observe that, if we divide the family of generators $T=\left\{T_{m}^{(a)}(u) \mid(a, m\right.$, $u) \in H\}$ of $\widetilde{\mathcal{T}}_{\ell}\left(A_{r}\right)$ into two subfamilies, $T_{e}=\left\{T_{m}^{(a)}(u) \mid(a, m, u) \in H_{e}\right\}$ and 
$T_{o}=\left\{T_{m}^{(a)}(u) \mid(a, m, u) \in H_{o}\right\}$, then $T_{e}$ and $T_{o}$ have no mutual relation in $\widetilde{\mathbb{T}}_{\ell}\left(X_{r}\right)$. Therefore, to prove Theorem 5.3, it is enough to consider the halffamily $T_{e}$ of $T$ (cf. (4.34)). Then, to prove Theorem 5.3, we have only to show the following.

Proposition 5.8. There exists some $\ell \times \infty$ matrix $M=\left[x_{k}\right]_{k \in \mathbb{Z}}$ over $\widetilde{\mathcal{T}}_{\ell}\left(A_{r}\right)$ satisfying the condition (5.4) such that, for $D_{M}=\left\{D_{m}^{(a)}(u) \mid(a, m\right.$, $\left.u) \in H_{e}\right\}$, the following relation holds in $\widetilde{\mathfrak{T}}_{\ell}\left(A_{r}\right)$ :

$$
T_{m}^{(a)}(u)=D_{m}^{(a)}(u) \quad\left((a, m, u) \in H_{e}\right) .
$$

Proof. We define $x_{0}, \ldots, x_{r+\ell} \in\left(\widetilde{\mathcal{T}}_{\ell}\left(A_{r}\right)\right)^{\ell}$ as follows. First, let us arbitrarily choose $x_{1}, \ldots, x_{\ell-1}$ such that

$$
D_{0}^{(0)}(0)=\operatorname{det}\left[x_{0} \cdots x_{\ell-1}\right]=T_{0}^{(0)}(0)=T_{0}^{(1)}(1)
$$

holds. We define $x_{\ell}$ by

$$
x_{\ell}=\frac{1}{T_{0}^{(1)}(1)} \sum_{m=0}^{\ell-1}(-1)^{\ell-1-m} T_{m}^{(1)}(-1-m) x_{m} .
$$

Then, the following equality holds:

$$
D_{m}^{(1)}(-1-m)=T_{m}^{(1)}(-1-m) \quad(m=0, \ldots, \ell-1) .
$$

For example,

$$
\begin{aligned}
D_{0}^{(1)}(-1) & =\operatorname{det}\left[x_{1} \cdots x_{\ell}\right] \\
& =\frac{(-1)^{\ell-1} T_{0}^{(1)}(-1)}{T_{0}^{(1)}(1)} \operatorname{det}\left[x_{1} \cdots x_{\ell-1} x_{0}\right]=T_{0}^{(1)}(-1) .
\end{aligned}
$$

Similarly, we recursively define the rest, $x_{\ell+1}, \ldots, x_{r+\ell}$, by

$$
\begin{aligned}
x_{\ell+j}=\frac{1}{T_{0}^{(1)}(1-2 j)} \sum_{m=0}^{\ell-1}(-1)^{\ell-1-m} T_{m}^{(1)}(-1-2 j-m) x_{j+m} & \\
& (j=1, \ldots, r)
\end{aligned}
$$

so that the following equality holds (including (5.13) as $j=0$ ): 


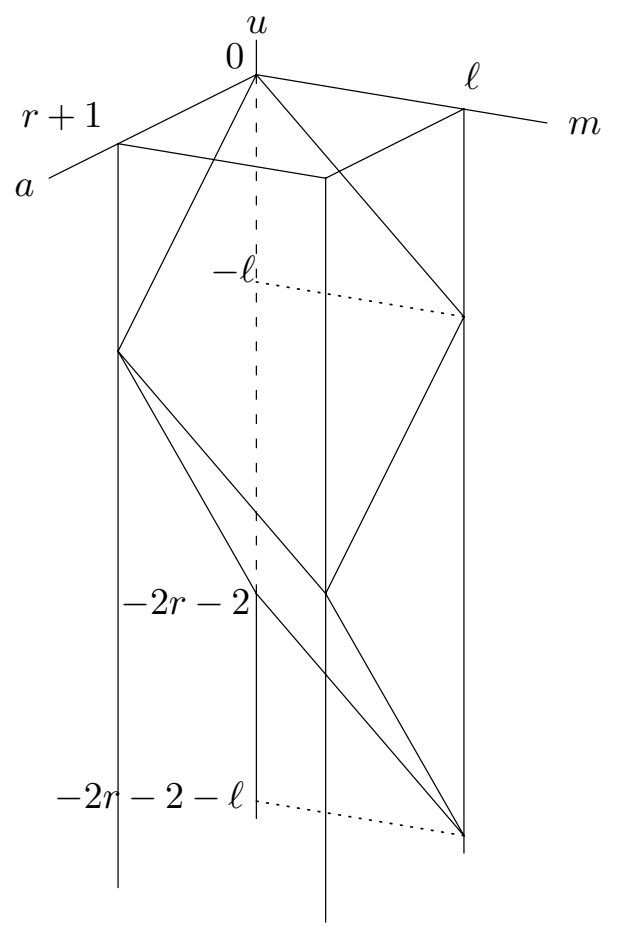

Figure 4: The prism $P$ defined in (5.17)

$$
\begin{aligned}
D_{m}^{(1)}(-1-2 j-m)=T_{m}^{(1)}(-1-2 j-m) & \\
& (m=0, \ldots, \ell-1 ; j=0, \ldots, r) .
\end{aligned}
$$

Finally, we define the matrix $M=\left[x_{k}\right]_{k \in \mathbb{Z}}$ by extending the above $x_{0}, \ldots$, $x_{r+\ell}$ with (5.4).

For $D_{M}$, we claim that the relation (5.10) holds in $\widetilde{\mathcal{T}}_{\ell}\left(A_{r}\right)$. This will be shown inductively, based on the fact that the T-system and the spiral boundary condition are satisfied by both $T_{e}$ and $D_{M}$.

To proceed the induction, it is convenient to introduce a prism $P$, where

$$
P=\{(a, m, u) \in H \mid a+m+u \leq 0, a-m-u \leq 2 r+2\}
$$

(see Figure 4). We use the notations $P_{e}=P \cap H_{e}, P_{e}[a=1]=\{(a, m, u) \in$ $\left.P_{e} \mid a=1\right\}$, and so forth.

First, we show that (5.10) is true for $(a, m, u) \in P_{e}$ by the induction on a. By (5.11), (5.16), and the spiral boundary condition, we see that (5.10) 
is true for any $(a, m, u)$ in the set

$$
P_{e}[a=0] \cup P_{e}[a=1] \cup P_{e}[m=0] \cup P_{e}[m=\ell] .
$$

Assume that (5.10) is true up to $a$. By (5.2), we have

$$
T_{m}^{(a+1)}(u)=\frac{1}{T_{m}^{(a-1)}(u)}\left(T_{m}^{(a)}(u-1) T_{m}^{(a)}(u+1)-T_{m-1}^{(a)}(u) T_{m+1}^{(a)}(u)\right) .
$$

On the other hand, by (5.7) and the induction hypothesis, we have

$$
D_{m}^{(a+1)}(u)=\frac{1}{T_{m}^{(a-1)}(u)}\left(T_{m}^{(a)}(u-1) T_{m}^{(a)}(u+1)-T_{m-1}^{(a)}(u) T_{m+1}^{(a)}(u)\right) .
$$

Thus, the relation $D_{m}^{(a+1)}(u)=T_{m}^{(a+1)}(u)$ is obtained. Therefore, (5.10) is true for $(a, m, u) \in P_{e}$.

Next, we show that $(5.10)$ is true for any $(a, m, u) \in H_{e} \backslash P_{e}$. We first remark that, by the spiral boundary condition, (5.10) is now true for any $(a, m, u) \in \partial H_{e}$. Then, using the T-system (5.2) once again as

$$
T_{m}^{(a)}(u \pm 1)=\frac{1}{T_{m}^{(a)}(u \mp 1)}\left(T_{m-1}^{(a)}(u) T_{m+1}^{(a)}(u)+T_{m}^{(a-1)}(u) T_{m}^{(a+1)}(u)\right),
$$

and repeating the same argument as before, one can inductively, with respect to $\pm u$, conclude that $(5.10)$ is true for any $(a, m, u) \in H_{e} \backslash P_{e}$.

This completes the proof of Theorem 5.3.

\subsection{Periodicity of Y-system}

The periodicity of $y_{\ell}\left(A_{r}\right)$ follows from that of $\widetilde{\mathcal{T}}_{\ell}\left(A_{r}\right)$. This is due to the fact that, unlike the unit boundary condition, the spiral boundary condition is fully compatible with the restriction of the Y-system in view of Theorem 2.12 (cf. Proposition 3.8). Namely, we have the following.

Proposition 5.9. (1) There is a ring homomorphism

$$
\tilde{\varphi}_{\ell}: y_{\ell}\left(A_{r}\right) \rightarrow \widetilde{\mathcal{T}}_{\ell}\left(A_{r}\right)
$$

defined by

$$
Y_{m}^{(a)}(u) \mapsto \frac{T_{m}^{(a-1)}(u) T_{m}^{(a+1)}(u)}{T_{m-1}^{(a)}(u) T_{m+1}^{(a)}(u)} \quad\left((a, m, u) \in H^{\circ}\right)
$$


(2) There is a ring homomorphism

$$
\psi_{\ell}: \widetilde{\mathcal{T}}_{\ell}\left(A_{r}\right) \rightarrow y_{\ell}\left(A_{r}\right)
$$

such that $\psi_{\ell} \circ \widetilde{\varphi}_{\ell}=\operatorname{id} y_{\ell}\left(X_{r}\right)$.

Proof. (1) It is enough to check the compatibility between the boundary conditions of $\widetilde{\mathbb{T}}_{\ell}\left(A_{r}\right)$ and $\mathbb{Y}_{\ell}\left(A_{r}\right)$ as Theorem 2.12 and Proposition 3.8. For example, to see the compatibility with $Y_{0}^{(a)}(u)^{-1}=0(a \neq 0)$, we formally extend (5.2) to $m=0$ as

$$
T_{0}^{(a)}(u-1) T_{0}^{(a)}(u+1)=T_{-1}^{(a)}(u) T_{1}^{(a)}(u)+T_{0}^{(a-1)}(u) T_{0}^{(a+1)}(u)
$$

and use (5.3). Then we have $T_{-1}^{(a)}(u)=0$. The other cases are checked similarly.

(2) We define the image $\psi_{\ell}\left(T_{m}^{(a)}(u)\right)$ for the half-family $T_{e}$ of $T$. The other half, $T_{o}$, is completely parallel. The construction is in four steps and similar to the one for Theorem 2.12. For simplicity, we write the image $\psi_{\ell}\left(T_{m}^{(a)}(u)\right)$ as $T_{m}^{(a)}(u)$. Let $P$ be the prism defined in (5.17).

Step 1. We arbitrarily choose $T_{0}^{(0)}(-2 j) \in \mathrm{y}_{\ell}\left(A_{r}\right)^{\times}(j=0,1, \ldots, r+1)$. Then, we define $T_{m}^{(a)}(u)$ for the rest of $(a, m, u) \in P_{e}[a=0] \cup P_{e}[m=0] \cup$ $P_{e}[m=\ell]$ by $(5.8)$.

Step 2. We arbitrarily choose $T_{m}^{(1)}(-1-m) \in y_{\ell}\left(A_{r}\right)^{\times}(m=1, \ldots, \ell-$ $1)$. Then, we recursively, with respect to $u$, define $T_{m}^{(a)}(u)$ for the rest of $(a, m, u) \in P_{e}[a=1]$ by

$$
T_{m}^{(1)}(u-1)=\left(1+Y_{m}^{(1)}(u)\right) \frac{T_{m-1}^{(1)}(u) T_{m+1}^{(1)}(u)}{T_{m}^{(1)}(u+1)} .
$$

Step 3. We recursively, with respect to $a$, define $T_{m}^{(a)}(u)$ for the rest of $(a, m, u) \in P_{e}$ by

$$
T_{m}^{(a+1)}(u)=\frac{1}{1+Y_{m}^{(a)}(u)^{-1}} \frac{T_{m}^{(a)}(u-1) T_{m}^{(a)}(u+1)}{T_{m}^{(a-1)}(u)} .
$$

Step 4. We define $T_{m}^{(a)}(u)$ for the rest of $(a, m, u) \in \partial H_{e}$ by (5.8). Then, we recursively, with respect to $\pm u$, define $T_{m}^{(a)}(u)$ for the rest of $(a, m, u) \in H_{e}$ by

$$
T_{m}^{(a)}(u \pm 1)=\left(1+Y_{m}^{(a)}(u)^{-1}\right) \frac{T_{m}^{(a-1)}(u) T_{m}^{(a+1)}(u)}{T_{m}^{(a)}(u \mp 1)} .
$$


Claim. The family $\left\{T_{m}^{(a)}(u) \mid(a, m, u) \in H_{e}\right\}$ defined above satisfies the following relations in $y_{\ell}\left(A_{r}\right)$ :

$$
\begin{aligned}
1+Y_{m}^{(a)}(u) & =\frac{T_{m}^{(a)}(u-1) T_{m}^{(a)}(u+1)}{T_{m-1}^{(a)}(u) T_{m+1}^{(a)}(u)} \quad\left((a, m, u) \in H_{o}^{\circ}\right), \\
1+Y_{m}^{(a)}(u)^{-1} & =\frac{T_{m}^{(a)}(u-1) T_{m}^{(a)}(u+1)}{T_{m}^{(a-1)}(u) T_{m}^{(a+1)}(u)} \quad\left((a, m, u) \in H_{o}^{\circ}\right) .
\end{aligned}
$$

The relation (5.29) clearly holds by the definition of $T_{m}^{(a)}(u)$ in (5.26) and (5.27). With (5.26), one can verify that (5.28) is true for any $(a, m, u) \in$ $P_{o}^{\circ}:=P \cap H_{o}^{\circ}$ by induction with respect to $a$. Then, in a similar way, with (5.27), one can prove that (5.28) is true for the rest of $(a, m, u) \in H_{o}^{\circ}$ by induction with respect to $u$. This completes the proof of the claim.

Now, taking the inverse sum of (5.28) and (5.29), we obtain (5.2). Therefore, $\psi_{\ell}$ is a ring homomorphism. Furthermore, taking the ratio of (5.28) and (5.29), we obtain $Y_{m}^{(a)}(u)=T_{m}^{(a-1)}(u) T_{m}^{(a+1)}(u) /\left(T_{m-1}^{(a)}(u) T_{m+1}^{(a)}(u)\right)$. This proves $\psi_{\ell} \circ \widetilde{\varphi}_{\ell}=\operatorname{id}_{y_{\ell}}\left(A_{r}\right)$.

By Theorem 5.3 and Proposition 5.9, we obtain the following.

Corollary 5.10 (cf. Volkov [V, Theorem 1]). The following relations hold in $y_{\ell}\left(A_{r}\right)$ :

(1) Half-periodicity: $Y_{m}^{(a)}(u+r+1+\ell)=Y_{\ell-m}^{(r+1-a)}(u)$.

(2) Periodicity: $Y_{m}^{(a)}(u+2(r+1+\ell))=Y_{m}^{(a)}(u)$.

By Proposition 5.9, we also obtain the following.

Corollary 5.11 (cf. Volkov [V, Proposition 1]). For any ring $R$, the map

$$
\widetilde{\varphi}_{\ell}^{*}: \operatorname{Hom}\left(\widetilde{\mathcal{T}}_{\ell}\left(A_{r}\right), R\right) \rightarrow \operatorname{Hom}\left(y_{\ell}\left(A_{r}\right), R\right),
$$

induced from the homomorphism $\tilde{\varphi}_{\ell}$ in (5.22), is surjective.

\section{§6. Determinant method II: $\mathcal{T}_{\ell}\left(C_{r}\right)$}

In this section we prove the periodicity of $\mathcal{T}_{\ell}\left(C_{r}\right)$ for any $\ell \geq 2$. We do it in three steps. First, we introduce a ring $\widetilde{\mathcal{T}}_{\ell}\left(C_{r}\right)$ by slightly generalizing the unit boundary condition of $\mathcal{T}_{\ell}\left(C_{r}\right)$. Second, we show $\widetilde{\mathcal{T}}_{\ell}\left(C_{r}\right)$ is isomorphic to another ring $\widehat{\mathcal{T}}_{2 \ell}\left(A_{2 r+1}\right)$, which is a variant of $\mathcal{T}_{2 \ell}\left(A_{2 r+1}\right)$. Last, we apply the determinant method to $\widehat{\mathcal{T}}_{2 \ell}\left(A_{2 r+1}\right)$. 


\subsection{Level $\ell$ restricted T-system with quasi-unit boundary con- dition}

For $X_{r}=C_{r}$, let $t_{a}(a \in I)$ be the number in $(2.1)$, that is, $t_{a}=2$ for $a=1, \ldots, r-1$ and 1 for $a=r$.

We find that it is convenient to generalize the problem slightly as follows.

Definition 6.1. Fix an integer $\ell \geq 2$. The level $\ell$ restricted T-system $\widetilde{\mathbb{T}}_{\ell}\left(C_{r}\right)$ of type $C_{r}$ with the quasi-unit boundary condition is the following system of relations for a family of variables $T=\left\{T_{m}^{(a)}(u) \mid a=1, \ldots, r ; m=\right.$ $\left.1, \ldots, t_{a} \ell-1 ; u \in(1 / 2) \mathbb{Z}\right\} \cup\left\{T_{\ell}^{(r)}(u) \mid u \in(1 / 2) \mathbb{Z}\right\}$.

(1) T-system (2.4):

$$
\begin{aligned}
& T_{m}^{(a)}\left(u-\frac{1}{2}\right) T_{m}^{(a)}\left(u+\frac{1}{2}\right)=T_{m-1}^{(a)}(u) T_{m+1}^{(a)}(u) \\
& +T_{m}^{(a-1)}(u) T_{m}^{(a+1)}(u) \\
& \left(a=1, \ldots, r-2 ; m=1, \ldots, 2 \ell-1 ; u \in \frac{1}{2} \mathbb{Z}\right), \\
& T_{2 m}^{(r-1)}\left(u-\frac{1}{2}\right) T_{2 m}^{(r-1)}\left(u+\frac{1}{2}\right)=T_{2 m-1}^{(r-1)}(u) T_{2 m+1}^{(r-1)}(u) \\
& +T_{2 m}^{(r-2)}(u) T_{m}^{(r)}\left(u-\frac{1}{2}\right) T_{m}^{(r)}\left(u+\frac{1}{2}\right) \\
& \left(m=1, \ldots, \ell-1 ; u \in \frac{1}{2} \mathbb{Z}\right), \\
& T_{2 m+1}^{(r-1)}\left(u-\frac{1}{2}\right) T_{2 m+1}^{(r-1)}\left(u+\frac{1}{2}\right)=T_{2 m}^{(r-1)}(u) T_{2 m+2}^{(r-1)}(u) \\
& +T_{2 m+1}^{(r-2)}(u) T_{m}^{(r)}(u) T_{m+1}^{(r)}(u) \\
& \left(m=0, \ldots, \ell-1 ; u \in \frac{1}{2} \mathbb{Z}\right), \\
& T_{m}^{(r)}(u-1) T_{m}^{(r)}(u+1)=T_{m-1}^{(r)}(u) T_{m+1}^{(r)}(u)+T_{2 m}^{(r-1)}(u) \\
& \left(m=1, \ldots, \ell-1 ; u \in \frac{1}{2} \mathbb{Z}\right) .
\end{aligned}
$$

(2) Quasi-unit boundary condition:

$$
\begin{aligned}
& T_{m}^{(0)}(u)=T_{0}^{(a)}(u)=1, \\
& T_{2 \ell}^{(a)}(u)=1 \quad(a=1, \ldots, r-1),
\end{aligned}
$$

if they occur in the right-hand sides of the relations, and

$$
T_{\ell}^{(r)}(u)^{2}=1, \quad T_{\ell}^{(r)}(u+1)=T_{\ell}^{(r)}(u) .
$$


Definition 6.2. Let $\widetilde{\mathcal{T}}_{\ell}\left(C_{r}\right)$ be the ring with generators $T_{m}^{(a)}(u)^{ \pm 1}(a=$ $\left.1, \ldots, r ; m=1, \ldots, t_{a} \ell-1 ; u \in(1 / 2) \mathbb{Z}\right), T_{\ell}^{(r)}(u)(u \in(1 / 2) \mathbb{Z})$ and the relations $\widetilde{\mathbb{T}}_{\ell}\left(C_{r}\right)$.

In (6.7), if we impose $T_{\ell}^{(r)}(u)=1(u \in(1 / 2) \mathbb{Z})$, then $\widetilde{\mathbb{T}}_{\ell}\left(C_{r}\right)$ reduces to $\mathbb{T}_{\ell}\left(C_{r}\right)$. In other words, $\mathcal{T}_{\ell}\left(C_{r}\right)$ is isomorphic to $\tilde{\mathcal{T}}_{\ell}\left(C_{r}\right) / J$, where $J$ is the ideal of $\widetilde{\mathcal{T}}_{\ell}\left(C_{r}\right)$ generated by $T_{\ell}^{(r)}(u)-1(u \in(1 / 2) \mathbb{Z})$.

Recall that the dual Coxeter number of $C_{r}$ is $r+1$. We will prove the following.

THEOREM 6.3. The following relations hold in $\widetilde{\mathcal{T}}_{\ell}\left(C_{r}\right)$ :

(1) Half-periodicity: $T_{m}^{(a)}(u+r+1+\ell)= \begin{cases}T_{2 \ell-m}^{(a)}(u) & a=1, \ldots, r-1, \\ T_{\ell}^{(r)}(u) T_{\ell-m}^{(r)}(u) & a=r .\end{cases}$

(2) Periodicity: $T_{m}^{(a)}(u+2(r+1+\ell))=T_{m}^{(a)}(u)$.

By the above remark, we obtain the following.

COROLlary 6.4. The following relations hold in $\mathcal{T}_{\ell}\left(C_{r}\right)$.

(1) Half-periodicity: $T_{m}^{(a)}(u+r+1+\ell)=T_{t_{a} \ell-m}^{(a)}(u)$.

(2) Periodicity: $T_{m}^{(a)}(u+2(r+1+\ell))=T_{m}^{(a)}(u)$.

6.2. System $\widehat{\mathbb{T}}_{2 \ell}\left(A_{2 r+1}\right)$

To prove Theorem 6.3 , we introduce another system of relations that is equivalent to $\widetilde{\mathbb{T}}_{\ell}\left(C_{r}\right)$.

Let us set

$$
\begin{aligned}
\widehat{H} & =\left\{(a, m, u) \mid a=0, \ldots, 2 r+2 ; m=0, \ldots, 2 \ell ; u \in \frac{1}{2} \mathbb{Z}\right\}, \\
\widehat{H}^{\circ} & =\{(a, m, u) \in \widehat{H} \mid a \neq 0,2 r+2 ; m \neq 0,2 \ell\}, \\
\partial \widehat{H} & =\widehat{H} \backslash \widehat{H}^{\circ} \\
\widehat{H}_{e} & =\{(a, m, u) \in \widehat{H} \mid a+m+2 u \text { is even }\}, \\
\widehat{H}_{o} & =\{(a, m, u) \in \widehat{H} \mid a+m+2 u \text { is odd }\} .
\end{aligned}
$$

We again use the combined notations $\widehat{H}_{o}^{\circ}=\widehat{H}^{\circ} \cap \widehat{H}_{o}, \partial \widehat{H}_{e}=\partial \widehat{H} \cap \widehat{H}_{e}$, and so forth.

Definition 6.5. Fix an integer $\ell \geq 2$. The level $2 \ell$ restricted $T$-system $\widehat{\mathbb{T}}_{2 \ell}\left(A_{2 r+1}\right)$ of type $A_{2 r+1}$ with the quasi-symmetric condition is the following system of relations for a family of variables $S=\left\{S_{m}^{(a)}(u) \mid(a, m, u) \in \widehat{H}^{\circ}\right\}$. 
(1) T-system:

$$
S_{m}^{(a)}\left(u-\frac{1}{2}\right) S_{m}^{(a)}\left(u+\frac{1}{2}\right)=S_{m-1}^{(a)}(u) S_{m+1}^{(a)}(u)+S_{m}^{(a-1)}(u) S_{m}^{(a+1)}(u) .
$$

(2) Quasi-symmetric condition:

$$
S_{m}^{(a)}(u)=(-1)^{m} S_{m}^{(2 r+2-a)}(u) .
$$

In particular, $S_{m}^{(r+1)}(u)=0$ for odd $m$.

(3) Quasi-symmetric unit boundary condition:

$$
\begin{aligned}
S_{0}^{(a)}(u) & =S_{2 \ell}^{(a)}(u)=S_{m}^{(0)}(u)=1, \\
S_{m}^{(2 r+2)}(u) & =(-1)^{m},
\end{aligned}
$$

if they occur in the right-hand side of the relations.

Definition 6.6. Let $\widehat{\mathcal{T}}_{2 \ell}\left(A_{2 r+1}\right)$ be the ring with generators $S_{m}^{(a)}(u)^{ \pm 1}$ $\left((a, m, u) \in \widehat{H}^{\circ},(a, m) \neq(r+1\right.$,odd $\left.)\right)$ and the relations $\widehat{\mathbb{T}}_{2 \ell}\left(A_{2 r+1}\right)$.

REMARK 6.7. The system $\widehat{\mathbb{T}}_{2 \ell}\left(A_{2 r+2}\right)$ and the map $\rho$ below are the restricted versions of those considered in [KOSY, Section 4].

Proposition 6.8. There is a ring isomorphism

$$
\rho: \widehat{\mathcal{T}}_{2 \ell}\left(A_{2 r+1}\right) \stackrel{\sim}{\rightarrow} \widetilde{\mathcal{T}}_{\ell}\left(C_{r}\right)
$$

defined by

$$
\begin{aligned}
S_{m}^{(a)}(u) & \mapsto T_{m}^{(a)}(u) \quad(a=1, \ldots, r-1), \\
S_{2 m}^{(r)}(u) & \mapsto T_{m}^{(r)}\left(u-\frac{1}{2}\right) T_{m}^{(r)}\left(u+\frac{1}{2}\right), \\
S_{2 m+1}^{(r)}(u) & \mapsto T_{m}^{(r)}(u) T_{m+1}^{(r)}(u), \\
S_{2 m}^{(r+1)}(u) & \mapsto T_{m}^{(r)}(u)^{2}, \\
\rho\left(S_{m}^{(a)}(u)\right) & =(-1)^{m} \rho\left(S_{m}^{(2 r+2-a)}(u)\right) \quad(a=r+2, \ldots, 2 r+1),
\end{aligned}
$$

where $T_{0}^{(r)}(u)=1$. 
Proof. It is easy to check that the map $\rho$ is a ring homomorphism by substitution (cf. [KOSY, Proposition 4.3]). For simplicity, we write the image $\rho\left(S_{m}^{(a)}(u)\right)$ as $S_{m}^{(a)}(u)$. Then, for example, to show

$$
\begin{aligned}
S_{2 m}^{(r)}\left(u-\frac{1}{2}\right) S_{2 m}^{(r)}\left(u+\frac{1}{2}\right)= & S_{2 m-1}^{(r)}(u) S_{2 m+1}^{(r)}(u) \\
& +S_{2 m}^{(r-1)}(u) S_{2 m}^{(r+1)}(u)
\end{aligned}
$$

multiply $(6.4)$ by $T_{m}^{(r)}(u)^{2}$, and then use (6.19)-(6.21).

Let us consider the inverse map $\rho^{-1}: \widetilde{\mathcal{T}}_{\ell}\left(C_{r}\right) \rightarrow \widehat{\mathcal{T}}_{2 \ell}\left(A_{2 r+1}\right)$. Looking at (6.18) and (6.20), it should be given by the following correspondence:

$$
\begin{aligned}
& T_{m}^{(a)}(u) \mapsto S_{m}^{(a)}(u) \quad(a=1, \ldots, r-1), \\
& T_{m}^{(r)}(u) \mapsto \begin{cases}\frac{S_{2 m-1}^{(r)}(u) S_{2 m-5}^{(r)}(u) \cdots S_{3}^{(r)}(u)}{S_{2 m-3}^{(r)}(u) S_{2 m-7}^{(r)}(u) \cdots S_{1}^{(r)}(u)} & (m: \text { even }), \\
\frac{S_{2 m-1}^{(r)}(u) S_{2 m-5}^{(r)}(u) \cdots S_{1}^{(r)}(u)}{S_{2 m-3}^{(r)}(u) S_{2 m-7}^{(r)}(u) \cdots S_{3}^{(r)}(u)} & (m: \text { odd }) .\end{cases}
\end{aligned}
$$

For simplicity, we write the image $\rho^{-1}\left(T_{m}^{(a)}(u)\right)$ as $T_{m}^{(a)}(u)$.

Claim. The family $T=\left\{T_{m}^{(a)}(u)\right\}$ above satisfies the following relations in $\widehat{\mathcal{T}}_{2 \ell}\left(A_{2 r+1}\right)$ :

$$
\begin{array}{rlrl}
T_{m}^{(r)}\left(u-\frac{1}{2}\right) T_{m}^{(r)}\left(u+\frac{1}{2}\right) & =S_{2 m}^{(r)}(u) & & (m=1, \ldots, \ell) \\
T_{m}^{(r)}(u) T_{m+1}^{(r)}(u) & =S_{2 m+1}^{(r)}(u) & (m=1, \ldots, \ell-1) \\
T_{m}^{(r)}(u)^{2} & =S_{2 m}^{(r+1)}(u) & (m=1, \ldots, \ell)
\end{array}
$$

where $S_{2 \ell}^{(r)}(u)=S_{2 \ell}^{(r+1)}(u)=1$.

Indeed, (6.27) follows immediately from (6.25), while (6.26) and (6.28) are proved by the induction with respect to $m$.

Now, it is easy to check that $\rho^{-1}$ is a ring homomorphism by substitution.

By comparing (6.18)-(6.21) with $(6.24),(6.26)-(6.28)$, we see that $\rho^{-1}$ is the inverse of $\rho$.

The following theorem is an analogue of Theorem 5.3.

THEOREM 6.9. The following relations hold in $\widehat{\mathcal{T}}_{2 \ell}\left(A_{2 r+1}\right)$. 
(1) Half-periodicity: $S_{m}^{(a)}(u+r+1+\ell)=(-1)^{m} S_{2 \ell-m}^{(2 r+2-a)}(u)=S_{2 \ell-m}^{(a)}(u)$.

(2) Periodicity: $S_{m}^{(a)}(u+2(r+1+\ell))=S_{m}^{(a)}(u)$.

A proof of Theorem 6.9 by the determinant method will be given in Section 6.3.

Admitting Theorem 6.9, let us prove Theorem 6.3 first.

Proof of Theorem 6.3. It is enough to prove (1). For simplicity, we write the image $\rho\left(S_{m}^{(a)}(u)\right)$ as $S_{m}^{(a)}(u)$. By $(6.18)$, we immediately obtain the relation for $a=1, \ldots, r-1$. Let us verify the case $a=r$. By Proposition 6.8 and Theorem 6.9, we have (e.g., for $\ell, m$ : even)

$$
\begin{aligned}
& T_{m}^{(r)}(u+r+1+\ell) \\
= & \frac{S_{2 m-1}^{(r)}(u+r+1+\ell) S_{2 m-5}^{(r)}(u+r+1+\ell) \cdots S_{3}^{(r)}(u+r+1+\ell)}{S_{2 m-3}^{(r)}(u+r+1+\ell) S_{2 m-7}^{(r)}(u+r+1+\ell) \cdots S_{1}^{(r)}(u+r+1+\ell)} \\
(6.29)= & \frac{S_{2 \ell-2 m+1}^{(r)}(u) S_{2 \ell-2 m+5}^{(r)}(u) \cdots S_{2 \ell-3}^{(r)}(u)}{S_{2 \ell-2 m+3}^{(r)}(u) S_{2 \ell-2 m+7}^{(r)}(u) \cdots S_{2 \ell-1}^{(r)}(u)} \\
= & T_{\ell}^{(r)}(u) \frac{S_{2 \ell-2 m-1}^{(r)}(u) S_{2 \ell-2 m-5}^{(r)}(u) \cdots S_{3}^{(r)}(u)}{S_{2 \ell-2 m-3}^{(r)}(u) S_{2 \ell-2 m-7}^{(r)}(u) \cdots S_{1}^{(r)}(u)} \\
= & T_{\ell}^{(r)}(u) T_{\ell-m}^{(r)}(u) .
\end{aligned}
$$

\subsection{Proof of Theorem 6.9}

The outline of the proof is the same as for Theorem 5.3, but there are some points where extra caution is necessary due to the singularities $S_{m}^{(r+1)}(u)=0$ $(m$ : odd).

Let $R$ be any ring. Let us take an arbitrary $2 \ell \times \infty$ matrix $M$ over $R$ such that $M=\left[x_{k}\right]_{k \in \mathbb{Z}}, x_{k} \in\left(\widehat{\mathcal{T}}_{2 \ell}\left(A_{2 r+1}\right)\right)^{2 \ell}$ with the following periodicity:

$$
x_{k+2 r+2+2 \ell}=x_{k} .
$$

Let $D_{M}=\left\{D_{m}^{(a)}(u) \mid(a, m, u) \in \widehat{H}_{e}\right\}$ be a family of minors of $M$ defined by

$$
D_{m}^{(a)}(u)=\operatorname{det}[\overbrace{x_{\beta} x_{\beta+1} \cdots}^{m} \overbrace{}^{\mathrm{v} \ldots \vee} \overbrace{\ldots}^{2 \ell-m} \overbrace{\ldots \ldots x_{\beta+2 r+2 \ell+1}^{\vee}}^{2 r+2-a}],
$$




$$
\beta=-\frac{a+m+2 u}{2} \in \mathbb{Z}
$$

Then, as Proposition 5.6, we have the following.

Proposition 6.10. The family $D_{M}=\left\{D_{m}^{(a)}(u) \mid(a, m, u) \in \widehat{H}_{e}\right\}$ satisfies the following relations in $R$.

(1) T-system:

$$
\begin{aligned}
& D_{m}^{(a)}\left(u-\frac{1}{2}\right) D_{m}^{(a)}\left(u+\frac{1}{2}\right)= D_{m-1}^{(a)}(u) D_{m+1}^{(a)}(u) \\
&+D_{m}^{(a-1)}(u) D_{m}^{(a+1)}(u) \\
& \quad\left((a, m, u) \in \widehat{H}_{o}^{\circ}\right),
\end{aligned}
$$

(2) Boundary condition:

$$
\begin{aligned}
D_{0}^{(a)}\left(u+\frac{1}{2}\right) & =D_{0}^{(a-1)}(u) \quad(a=1, \ldots, 2 r+2), \\
D_{2 \ell}^{(a)}\left(u+\frac{1}{2}\right) & =D_{2 \ell}^{(a+1)}(u) \quad(a=0, \ldots, 2 r+1), \\
D_{m}^{(0)}\left(u+\frac{1}{2}\right) & =D_{m+1}^{(0)}(u) \quad(m=0, \ldots, 2 \ell-1), \\
D_{m}^{(2 r+2)}\left(u+\frac{1}{2}\right) & =(-1) D_{m-1}^{(2 r+2)}(u) \quad(m=1, \ldots, 2 \ell) .
\end{aligned}
$$

(3) Half-periodicity:

$$
D_{m}^{(a)}(u+r+1+\ell)=(-1)^{m} D_{2 \ell-m}^{(2 r+2-a)}(u) .
$$

We consider an extended family of generators $S=\left\{S_{m}^{(a)}(u) \mid(a, m, u) \in\right.$ $\widehat{H}\}$ of $\widehat{\mathcal{T}}_{2 \ell}\left(A_{2 r+1}\right)$, where $S_{m}^{(r+1)}(u)=0(m$ : odd $)$ and $S_{m}^{(a)}(u)((a, m, u) \in$ $\partial \widehat{H})$ are given by (6.15) and (6.16). Again, we divide the family $S$ into two subfamilies, $S_{e}=\left\{S_{m}^{(a)}(u) \mid(a, m, u) \in \widehat{H}_{e}\right\}$ and $S_{o}=\left\{S_{m}^{(a)}(u) \mid(a, m, u) \in\right.$ $\left.\widehat{H}_{o}\right\}$, and we concentrate on the half-family $S_{e}$.

Theorem 6.9 follows from Proposition 6.10 and the following proposition.

Proposition 6.11. There exists some $2 \ell \times \infty$ matrix $M=\left\{x_{k}\right\}_{k \in \mathbb{Z}}$ satisfying the condition (6.30) such that, for $D_{M}=\left\{D_{m}^{(a)}(u) \mid(a, m, u) \in \widehat{H}_{e}\right\}$, the following relation holds in $\widehat{\mathcal{T}}_{2 \ell}\left(A_{2 r+1}\right)$ :

$$
S_{m}^{(a)}(u)=D_{m}^{(a)}(u) \quad\left((a, m, u) \in \widehat{H}_{e}\right) .
$$


Proof. We define $x_{0}, \ldots, x_{2 r+2 \ell+1} \in\left(\widehat{\mathcal{T}}_{2 \ell}\left(A_{2 r+1}\right)\right)^{2 \ell}$ as follows. First, let us arbitrarily choose $x_{1}, \ldots, x_{2 \ell-1}$ such that

$$
D_{0}^{(0)}(0):=\operatorname{det}\left[x_{0} \cdots x_{2 \ell-1}\right]=1=S_{0}^{(0)}(0)=S_{0}^{(1)}\left(\frac{1}{2}\right)
$$

holds. Second, we recursively define the rest, $x_{2 \ell}, \ldots, x_{2 r+2 \ell+1}$, by

$$
\begin{array}{r}
x_{2 \ell+j}=\frac{1}{S_{0}^{(1)}\left(\frac{1}{2}-j\right)} \sum_{m=0}^{2 \ell-1}(-1)^{2 \ell-1-m} S_{m}^{(1)}\left(-\frac{1+2 j+m}{2}\right) x_{j+m} \\
(j=0, \ldots, 2 r+1)
\end{array}
$$

so that the following equality holds:

$$
\begin{aligned}
D_{m}^{(1)}\left(-\frac{1+2 j+m}{2}\right)= & S_{m}^{(1)}\left(-\frac{1+2 j+m}{2}\right) \\
& (m=0, \ldots, 2 \ell-1 ; j=0, \ldots, 2 r+1) .
\end{aligned}
$$

Last, we define the matrix $M=\left[x_{k}\right]_{k \in \mathbb{Z}}$ by extending the above $x_{0}, \ldots$, $x_{2 r+2 \ell+1}$ with the condition (6.30).

For $D_{M}$, we claim that the relation (6.36) holds in $\widehat{\mathcal{T}}_{2 \ell}\left(A_{2 r+1}\right)$. This will be shown inductively, based on the fact that the T-system and the boundary condition are satisfied by both $S_{e}$ and $D_{M}$.

To proceed with the induction, we introduce a prism

$$
\widehat{P}=\{(a, m, u) \in \widehat{H} \mid a+m+2 u \leq 0, a-m-2 u \leq 4 r+4\} .
$$

We use the notations, $\widehat{P}_{e}=\widehat{P} \cap \widehat{H}_{e}, \widehat{P}_{e}[a=1]=\left\{(a, m, u) \in \widehat{P}_{e} \mid a=1\right\}$, and so on, once again.

First, we show that $(6.36)$ is true for $(a, m, u) \in \widehat{P}_{e}$ by the induction on $a$. By (6.37), (6.39), and (6.34), we see that (6.36) is true for any $(a, m, u)$ in the set

$$
\widehat{P}_{e}[a=0] \cup \widehat{P}_{e}[a=1] \cup \widehat{P}_{e}[m=0] \cup \widehat{P}_{e}[m=2 \ell] .
$$

Assume that (6.36) is true up to $a$. By the T-system (6.13), we have

$$
\begin{array}{r}
S_{m}^{(a+1)}(u)=\frac{1}{S_{m}^{(a-1)}(u)}\left(S_{m}^{(a)}\left(u-\frac{1}{2}\right) S_{m}^{(a)}\left(u+\frac{1}{2}\right)\right. \\
\left.-S_{m-1}^{(a)}(u) S_{m+1}^{(a)}(u)\right) .
\end{array}
$$


On the other hand, by (6.33) and the induction hypothesis, we have

$$
\begin{array}{r}
D_{m}^{(a+1)}(u)=\frac{1}{S_{m}^{(a-1)}(u)}\left(S_{m}^{(a)}\left(u-\frac{1}{2}\right) S_{m}^{(a)}\left(u+\frac{1}{2}\right)\right. \\
\left.-S_{m-1}^{(a)}(u) S_{m+1}^{(a)}(u)\right) .
\end{array}
$$

Thus, the relation $S_{m}^{(a+1)}(u)=D_{m}^{(a+1)}(u)$ is obtained. The induction works up to $a=r+2$. However, since $S_{2 m+1}^{(r+1)}(u)=D_{2 m+1}^{(r+1)}(u)=0(m=0, \ldots, \ell-1)$ by the assumption, the relation $S_{2 m+1}^{(r+3)}(u)=D_{2 m+1}^{(r+3)}(u)$ is not trivial. To overcome the point, we have to show the following claim:

Claim 1. For $(r+3,2 m+1, u) \in \widehat{P}_{e}$, the following relation holds in $\widehat{\mathcal{T}}_{2 \ell}\left(A_{2 r+1}\right)$ :

$$
D_{2 m+1}^{(r+3)}(u)=-D_{2 m+1}^{(r-1)}(u)
$$

Once the claim is verified, we have

$$
S_{2 m+1}^{(r+3)}(u)=-S_{2 m+1}^{(r-1)}(u)=-D_{2 m+1}^{(r-1)}(u)=D_{2 m+1}^{(r+3)}(u)
$$

so that the induction continues and completes.

Let us prove Claim 1. For simplicity, we write the ring $\widehat{\mathcal{T}}_{2 \ell}\left(A_{2 r+1}\right)$ as $R$. Consider a deformation $M_{\varepsilon}=\left[x_{k}+\varepsilon x_{k}^{\prime}\right]$ of the matrix $M$ with a formal parameter $\varepsilon$ and some $x_{k}^{\prime} \in R^{2 \ell}\left(k \in \mathbb{Z}, x_{k+2 r+2+2 \ell}^{\prime}=x_{k}^{\prime}\right)$. Let $D_{m}^{(a)}(u)_{\varepsilon} \in$ $R[\varepsilon]$ be the corresponding minor for $M_{\varepsilon}$. Fix $2 m+1$ and $u$ in (6.44). We choose $M_{\varepsilon}$ so that $D_{2 m+1}^{(r+1)}(u)_{\varepsilon}=\varepsilon D_{2 m+1}^{(r+1)}(u)^{\prime}+o(\varepsilon)$ with $D_{2 m+1}^{(r+1)}(u)^{\prime} \in R^{\times}$. (This is possible. For example, let $x^{\prime}$ be the $2 m+2$ th column for $D_{2 m+1}^{(r)}(u+$ $\left.\frac{1}{2}\right)$. Then, add $\varepsilon x^{\prime}$ to $M$ at the position of the last column for $D_{2 m+1}^{(r+1)}(u)$. This deformation yields $D_{2 m+1}^{(r+1)}(u)^{\prime}=D_{2 m+1}^{(r)}\left(u+\frac{1}{2}\right)=T_{2 m+1}^{(r)}\left(u+\frac{1}{2}\right) \in R^{\times}$.) Now, by (6.33), we have

$$
\begin{aligned}
D_{2 m+1}^{(r-1)}(u)_{\varepsilon} D_{2 m+1}^{(r+1)}(u)_{\varepsilon}= & D_{2 m+1}^{(r)}\left(u-\frac{1}{2}\right)_{\varepsilon} D_{2 m+1}^{(r)}\left(u+\frac{1}{2}\right)_{\varepsilon} \\
& -D_{2 m}^{(r)}(u)_{\varepsilon} D_{2 m+2}^{(r)}(u)_{\varepsilon}, \\
D_{2 m+1}^{(r+3)}(u)_{\varepsilon} D_{2 m+1}^{(r+1)}(u)_{\varepsilon}= & D_{2 m+1}^{(r+2)}\left(u-\frac{1}{2}\right)_{\varepsilon} D_{2 m+1}^{(r+2)}\left(u+\frac{1}{2}\right)_{\varepsilon} \\
& -D_{2 m}^{(r+2)}(u)_{\varepsilon} D_{2 m+2}^{(r+2)}(u)_{\varepsilon} .
\end{aligned}
$$


We take the ratio of the relations in $(6.45)$,

$$
\begin{aligned}
& \frac{D_{2 m+1}^{(r+3)}(u)_{\varepsilon}}{D_{2 m+1}^{(r-1)}(u)_{\varepsilon}} \\
& \quad=\frac{D_{2 m+1}^{(r+2)}\left(u-\frac{1}{2}\right)_{\varepsilon} D_{2 m+1}^{(r+2)}\left(u+\frac{1}{2}\right)_{\varepsilon}-D_{2 m}^{(r+2)}(u)_{\varepsilon} D_{2 m+2}^{(r+2)}(u)_{\varepsilon}}{D_{2 m+1}^{(r)}\left(u-\frac{1}{2}\right)_{\varepsilon} D_{2 m+1}^{(r)}\left(u+\frac{1}{2}\right)_{\varepsilon}-D_{2 m}^{(r)}(u)_{\varepsilon} D_{2 m+2}^{(r)}(u)_{\varepsilon}},
\end{aligned}
$$

which is in $R[[\varepsilon]]$. Let us calculate the right-hand side of (6.46). Let $\equiv$ mean the equality in $R[[\varepsilon]]$ modulo $\varepsilon R[[\varepsilon]]$. By $(6.33)$ and $D_{2 m+1}^{(r+1)}(u)_{\varepsilon} \equiv 0$, we have

$$
\begin{aligned}
D_{2 m}^{(r+2)}(u)_{\varepsilon} & \equiv D_{2 m}^{(r+1)}\left(u-\frac{1}{2}\right)_{\varepsilon} D_{2 m}^{(r+1)}\left(u+\frac{1}{2}\right)_{\varepsilon} / D_{2 m}^{(r)}(u)_{\varepsilon} \\
D_{2 m+2}^{(r+2)}(u)_{\varepsilon} & \equiv D_{2 m+2}^{(r+1)}\left(u-\frac{1}{2}\right)_{\varepsilon} D_{2 m+2}^{(r+1)}\left(u+\frac{1}{2}\right)_{\varepsilon} / D_{2 m+2}^{(r)}(u)_{\varepsilon} \\
D_{2 m+1}^{(r+2)}\left(u+\frac{1}{2}\right)_{\varepsilon} & \equiv-D_{2 m}^{(r+1)}\left(u+\frac{1}{2}\right)_{\varepsilon} D_{2 m+2}^{(r+1)}\left(u+\frac{1}{2}\right)_{\varepsilon} / D_{2 m+1}^{(r)}\left(u+\frac{1}{2}\right)_{\varepsilon} \\
D_{2 m+1}^{(r+2)}\left(u-\frac{1}{2}\right)_{\varepsilon} & \equiv-D_{2 m}^{(r+1)}\left(u-\frac{1}{2}\right)_{\varepsilon} D_{2 m+2}^{(r+1)}\left(u-\frac{1}{2}\right)_{\varepsilon} / D_{2 m+1}^{(r)}\left(u-\frac{1}{2}\right)_{\varepsilon} .
\end{aligned}
$$

Thanks to (6.47), the right-hand side of (6.46) equals, modulo $\varepsilon R[[\varepsilon]]$,

$$
(-1) \frac{D_{2 m}^{(r+1)}\left(u-\frac{1}{2}\right) D_{2 m+2}^{(r+1)}\left(u-\frac{1}{2}\right) D_{2 m}^{(r+1)}\left(u+\frac{1}{2}\right) D_{2 m+2}^{(r+1)}\left(u+\frac{1}{2}\right)}{D_{2 m+1}^{(r)}\left(u-\frac{1}{2}\right) D_{2 m+1}^{(r)}\left(u+\frac{1}{2}\right) D_{2 m}^{(r)}(u) D_{2 m+2}^{(r)}(u)} .
$$

Then, using the induction hypothesis (6.36) up to $a=r+2$ in $\widehat{P}_{e}$ and the relations (6.13) and (6.14), one can show that (6.48) is equal to -1 . Therefore, we have $D_{2 m+1}^{(r+3)}(u)_{\varepsilon} \equiv-D_{2 m+1}^{(r-1)}(u)_{\varepsilon}$, which means (6.44). This ends the proof of Claim 1.

Next, we show that $(6.36)$ is true for any $(a, m, u) \in \widehat{H}_{e} \backslash \widehat{P}_{e}$. We first remark that, by $(6.34),(6.36)$ is now true for any $(a, m, u) \in \partial \widehat{H}_{e}$. Then, using the T-system (6.13) as

$$
\begin{aligned}
S_{m}^{(a)}\left(u \pm \frac{1}{2}\right)=\frac{1}{S_{m}^{(a)}\left(u \mp \frac{1}{2}\right)} & \left(S_{m-1}^{(a)}(u) S_{m+1}^{(a)}(u)\right. \\
& \left.+S_{m}^{(a-1)}(u) S_{m}^{(a+1)}(u)\right)
\end{aligned}
$$

and repeating the same argument as before, one can inductively, with respect to $\pm u$, conclude that $(6.36)$ is true for any $(a, m, u) \in \widehat{H}_{e} \backslash \widehat{P}_{e}$. Once again, 
in the induction process we need the following claim, which can be verified by a similar deformation argument as Claim 1 .

Claim 2. For $(r+1,2 m+1, u) \in \widehat{H}_{e} \backslash \widehat{P}_{e}$, the following relation holds in $\widehat{\mathcal{T}}_{2 \ell}\left(A_{2 r+1}\right)$ :

$$
D_{2 m+1}^{(r+1)}(u)=0
$$

This ends the proof of Proposition 6.11.

REMARK 6.12. The system $\widetilde{\mathbb{T}}_{\ell}\left(C_{r}\right)$ plays a role analogous to that of $\widetilde{\mathbb{T}}_{\ell}\left(A_{r}\right)$ in the determinant method. Thus, it is natural to expect that the analogue of Proposition 5.9 also holds. Unfortunately, this is not true. The simplest case, $C_{2}$ and $\ell=2$, provides a counterexample by the similar reason to Example 3.9(3).

\section{§7. Direct method: $\mathcal{T}_{2}\left(A_{r}\right), \mathcal{T}_{2}\left(D_{r}\right)$, and $\mathcal{T}_{2}\left(B_{r}\right)$}

In this section we give some explicit formulae of generators $T_{m}^{(a)}(u)$ in terms of "initial variables" for $\mathcal{T}_{2}\left(A_{r}\right), \mathcal{T}_{2}\left(D_{r}\right)$, and $\mathcal{T}_{2}\left(B_{r}\right)$. Our goal here is to prove the periodicity for $\mathcal{T}_{2}\left(B_{r}\right)$. The formulae for $\mathcal{T}_{2}\left(A_{r}\right)$ and $\mathcal{T}_{2}\left(D_{r}\right)$ should be obtained as a specialization of the more general formulae by Caldero and Chapoton [CC, Theorem 3.4] and/or by Yang and Zelevinsky [YZ, Theorems 1.10, 1.12]. Nevertheless, we include these formulae as well, since they are good examples showing how the periodicity actually happens in the T-systems.

\subsection{Explicit formula by initial variables for $\mathcal{T}_{2}\left(A_{r}\right)$}

Throughout this subsection, $I=\{1, \ldots, r\}$ is the set enumerating the diagram $A_{r}$ in Figure 1. Recall that the (dual) Coxeter number of $A_{r}$ is $r+1$.

Definition 7.1. For a family of variables $X=\left\{x_{a} \mid a \in I\right\}$, we define $\gamma_{n}^{(j)}(X), \nu_{n}^{(j)}(X) \in \mathbb{Z}\left[X^{ \pm 1}\right](1 \leq j \leq r, 0 \leq n \leq j)$ as follows:

$$
\begin{aligned}
& \gamma_{n}^{(j)}(X)=\frac{1}{x_{n}}+\frac{x_{n-1}}{x_{n} x_{n+1}}+\frac{x_{n-1}}{x_{n+1} x_{n+2}}+\cdots+\frac{x_{n-1}}{x_{j-2} x_{j-1}}, \\
& \nu_{n}^{(j)}(X)=\frac{x_{n-1}}{x_{j-1}}
\end{aligned}
$$

where we set $x_{-1}=0$ and $x_{0}=1$. In particular,

$$
\gamma_{0}^{(j)}(X)=1, \quad \nu_{0}^{(j)}(X)=0
$$




$$
\gamma_{j}^{(j)}(X)=0, \quad \nu_{j}^{(j)}(X)=1 .
$$

The following lemma is easily checked.

LEMma 7.2. The following relations hold for any $1 \leq j \leq r$ and $1 \leq n \leq j$.

$$
\begin{aligned}
& x_{n-1} \gamma_{n-1}^{(j)}(X)=1+x_{n-2} \gamma_{n}^{(j)}(X), \\
& x_{j-1} \gamma_{n-1}^{(j)}(X)=x_{j-1} \gamma_{n-1}^{(j-1)}(X)+\nu_{n-1}^{(j-1)}(X) .
\end{aligned}
$$

We define a family $\tau=\left\{\tau^{(a)}(u ; X) \in \mathbb{Z}\left[X^{ \pm 1}\right] \mid a \in I, u \in \mathbb{Z}, a+u\right.$ is odd $\}$ as follows. First, we define, for $a \in I$ and $0 \leq k \leq r+2$,

$$
\tau^{(a)}(a-1+2 k ; X)=\left\{\begin{array}{c}
\gamma_{k}^{(a+k)}(X) x_{a+k}+\nu_{k}^{(a+k)}(X) \\
(0 \leq k \leq r-a), \\
\gamma_{r+1-a}^{(r)}(X)+\nu_{r+1-a}^{(r)}(X) \frac{1+x_{r-1}}{x_{r}} \\
\quad(k=r-a+1), \\
\gamma_{a+k-(r+2)}^{(k-1)}(X) x_{k-1}+\nu_{a+k-(r+2)}^{(k-1)}(X) \\
\quad(r-a+2 \leq k \leq r+1), \\
\gamma_{a}^{(r)}(X)+\nu_{a}^{(r)}(X) \frac{1+x_{r-1}}{x_{r}} \quad(k=r+2) .
\end{array}\right.
$$

Then, we extend the definition by the following periodicity:

$$
\tau^{(a)}(u+2(r+3) ; X)=\tau^{(a)}(u ; X) .
$$

By (7.3), we have

$$
\begin{gathered}
\tau^{(a)}(a-1 ; X)=x_{a}, \\
\tau^{(a)}(2 r+3-a ; X)=x_{r+1-a} .
\end{gathered}
$$

See Figure 5 for the fundamental domain of $\tau^{(a)}(u ; X)$ in the strip $\{(a, u) \mid$ $a \in I, u \in \mathbb{Z}, a+u$ is odd , which consists of four parts, corresponding to the cases in (7.7):

$$
\begin{aligned}
& D_{1}=\{(a, a-1+2 k) \mid a \in I, 0 \leq k \leq r-a\}, \\
& D_{2}=\{(a, a-1+2 k) \mid a \in I, k=r-a+1\}, \\
& D_{3}=\{(a, a-1+2 k) \mid a \in I, r-a+2 \leq k \leq r+1\}, \\
& D_{4}=\{(a, a-1+2 k) \mid a \in I, k=r+2\} .
\end{aligned}
$$




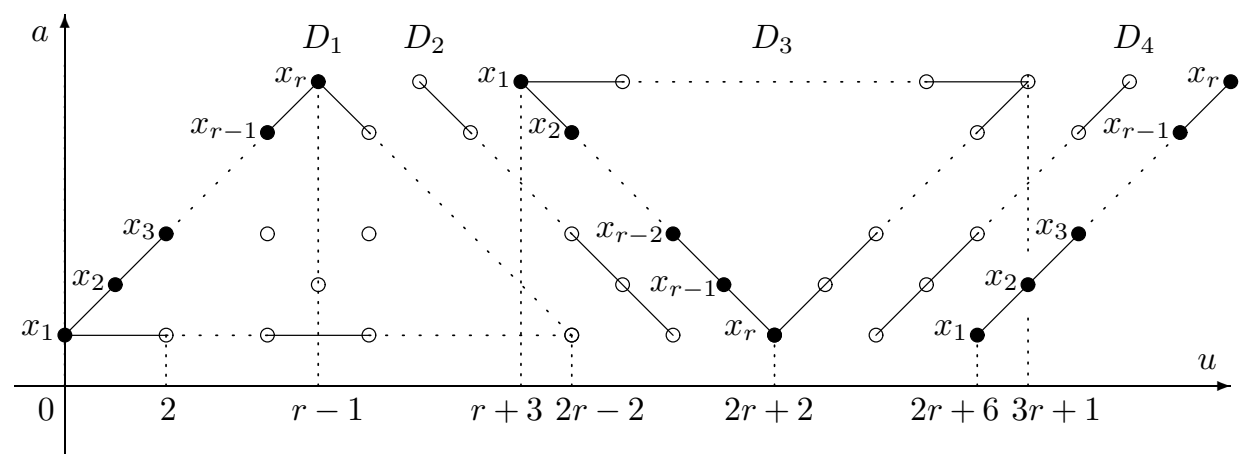

Figure 5: The fundamental domain of $\tau^{(a)}(u ; X)$ for $\mathcal{T}_{2}\left(A_{r}\right)$. The solid circles in $D_{1}$ are identified with the initial variables of

$$
\mathcal{T}_{2}\left(A_{r}\right)_{+} \cdot
$$

Proposition 7.3. The family $\tau$ satisfies the following relations in $\mathbb{Z}\left[X^{ \pm 1}\right]$.

(1) Half-periodicity:

$$
\tau^{(a)}(u+r+3 ; X)=\tau^{(r+1-a)}(u ; X) .
$$

(2) T-system $\mathbb{T}_{2}\left(A_{r}\right)$ :

$$
\tau^{(a)}(u-1 ; X) \tau^{(a)}(u+1 ; X)=1+\tau^{(a-1)}(u ; X) \tau^{(a+1)}(u ; X),
$$

where $\tau^{(0)}(u ; X)=\tau^{(r+1)}(u ; X)=1$ if they occur in the right-hand side.

Proof. For simplicity, in this proof we write $\gamma_{k}^{(j)}=\gamma_{k}^{(j)}(X), \nu_{k}^{(j)}=\nu_{k}^{(j)}(X)$, and $\tau^{(a)}(u)=\tau^{(a)}(u ; X)$.

(1) As an equivalent claim to (7.12), we prove

$$
\tau^{(a)}(u)=\tau^{(r+1-a)}(u+r+3) .
$$

Thanks to (7.8), it is enough to prove it for $(a, u) \in D_{1} \sqcup D_{2}$. Suppose that $(a, u)=(a, a-1+2 k) \in D_{1}$. Then we have

$$
\tau^{(a)}(a-1+2 k)=\gamma_{k}^{(a+k)} x_{a+k}+\nu_{k}^{(a+k)} .
$$

On the other hand, $(r+1-a, u+r+3)=\left(a^{\prime}, a^{\prime}-1+2 k^{\prime}\right) \in D_{3}$, where $a^{\prime}=r+1-a$ and $k^{\prime}=a+k+1$. Therefore, we have

$$
\begin{aligned}
\tau^{(r+1-a)}(u+r+3) & =\gamma_{a^{\prime}+k^{\prime}-(r+2)}^{\left(k^{\prime}-1\right)} x_{k^{\prime}-1}+\nu_{a^{\prime}+k^{\prime}-(r+2)}^{\left(k^{\prime}-1\right)} \\
& =\gamma_{k}^{(a+k)} x_{a+k}+\nu_{k}^{(a+k)}
\end{aligned}
$$


Thus the claim follows. The remaining case $(a, u) \in D_{2}$ is similar and easier.

(2) Thanks to (1), it is enough to show (7.13) for $(a, u-1)=(a, a-1+$ $2 k) \in D_{1} \sqcup D_{2}$; that is, $0 \leq k \leq r-a+1$. It is proved by a direct calculation using Lemma 7.2 .

Like (4.3), it is enough to consider the subring $\mathcal{T}_{2}\left(A_{r}\right)_{+}$of $\mathcal{T}_{2}\left(A_{r}\right)$ generated by $T_{1}^{(a)}(u)^{ \pm 1}(a \in I, u \in \mathbb{Z}, a+u$ : odd $)$. Set

$$
T^{\text {init }}=\left\{T_{1}^{(a)}(a-1) \mid a \in I\right\} .
$$

We call the elements of $T^{\text {init }}$ the initial variables of $\mathcal{T}_{2}\left(A_{r}\right)_{+}$.

By Proposition 7.3(2), we have the following explicit formula of other variables $T_{1}^{(a)}(u) \in \mathcal{T}_{2}\left(A_{r}\right)+$ by Laurent polynomials in the initial variables.

THEOREM 7.4. The following relation holds in $\mathcal{T}_{2}\left(A_{r}\right)_{+}$:

$$
T_{1}^{(a)}(u)=\tau^{(a)}\left(u ; T^{\text {init }}\right),
$$

where we set $x_{a}=T_{1}^{(a)}(a-1)(a \in I)$ in the right-hand side.

Proof. In the following we simply write $\tau^{(a)}(u)$ for $\tau^{(a)}\left(u ; T^{\text {init }}\right)$.

We rewrite $(7.18)$ as $(n \in \mathbb{Z})$,

$$
T_{1}^{(a)}(a-1+2 n)=\tau^{(a)}(a-1+2 n),
$$

and we show it by induction on $n$. Recall that $\mathbb{T}_{2}\left(A_{r}\right)$ becomes

$$
\begin{aligned}
T_{1}^{(a)} & (a-1+2 n) T_{1}^{(a)}(a+1+2 n) \\
& =1+T_{1}^{(a-1)}(a+2 n) T_{1}^{(a+1)}(a+2 n) .
\end{aligned}
$$

By (7.9), the $n=0$ case of $(7.19), \tau^{(a)}(a-1)=T_{1}^{(a)}(a-1)$, is satisfied. Assume that (7.19) holds up to $n(>0)$. Then (7.13) with $u=a+2 n$ becomes

$$
\begin{aligned}
& T_{1}^{(a)}(a-1+2 n) \tau^{(a)}(a-1+2(n+1)) \\
& \quad=1+\tau^{(a-1)}(a-2+2(n+1)) T_{1}^{(a+1)}(a+2 n) .
\end{aligned}
$$

By setting $a=1$ in (7.20) and (7.21), we obtain the relation

$$
\tau^{(1)}(2(n+1))=\frac{1+T_{1}^{(2)}(1+2 n)}{T_{1}^{(1)}(2 n)}=T^{(1)}(2(n+1)) .
$$


By increasing $a$ one by one up to $r$, we recursively obtain the relation

$$
\begin{aligned}
\tau^{(a)}(a-1+2(n+1)) & =\frac{1+T_{1}^{(a-1)}(a+2 n) T_{1}^{(a+1)}(a+2 n)}{T_{1}^{(a)}(a-1+2 n)} \\
& =T^{(a)}(a-1+2(n+1)) .
\end{aligned}
$$

The case of $n(<0)$ is similar.

In the above proof, we carefully stay inside the ring $\mathcal{T}_{2}\left(A_{r}\right)_{+}$by avoiding the division by $\tau^{(a)}(u)$ 's. As a corollary of Proposition 7.3(1) and Theorem 7.4 , we once again obtain the periodicity of $\mathcal{T}_{2}\left(A_{r}\right)$ (Corollary 4.5 for $\left.X_{r}=A_{r}\right)$.

\subsection{Explicit formula by initial variables for $\mathcal{T}_{2}\left(D_{r}\right)$}

The method is parallel to the $A_{r}$ case, so we present only the results for the most part. Throughout this subsection, $I=\{1, \ldots, r\}$ is the set enumerating the diagram $D_{r}$ in Figure 1. Recall that the (dual) Coxeter number of $D_{r}$ is $2 r-2$.

For a family of variables $X=\left\{x_{a} \mid a \in I\right\}$, we define $\gamma_{n}^{(j)}(X), \nu_{n}^{(j)}(X) \in$ $\mathbb{Z}\left[X^{ \pm 1}\right](1 \leq j \leq r-1,0 \leq n \leq j)$ by Definition 7.1 . We set

$$
\alpha_{n}=\gamma_{n}^{(r-1)}(X), \quad \beta_{n}=\nu_{n}^{(r-1)}(X), \quad z=x_{r-1} x_{r}
$$

Note that $\alpha_{r-1}=\beta_{0}=0, \alpha_{0}=\beta_{r-1}=1$, and $\beta_{1}=1 / x_{r-2}$.

Definition 7.5. Define $\Gamma_{n}^{(j)}(X), \Pi_{n}^{(j)}(X), \Omega_{n}^{(j)}(X) \in \mathbb{Z}\left[X^{ \pm 1}\right](0 \leq j \leq r-$ $2,0 \leq n \leq r-1-j)$ as

$$
\begin{aligned}
& \Gamma_{n}^{(j)}(X)=\alpha_{j} \alpha_{j+n} \\
& \Pi_{n}^{(j)}(X)=\alpha_{j} \beta_{j+n}+\beta_{j} \alpha_{j+n} \frac{2+\beta_{1}}{\beta_{1}}, \\
& \Omega_{n}^{(j)}(X)=\beta_{j} \beta_{j+n}\left(\frac{1+\beta_{1}}{\beta_{1}}\right)^{2} .
\end{aligned}
$$

In particular,

$$
\Gamma_{0}^{(j)}(X)=\alpha_{j}^{2}, \quad \Pi_{0}^{(j)}(X)=2 \alpha_{j} \beta_{j} \frac{1+\beta_{1}}{\beta_{1}}, \quad \Omega_{0}^{(j)}(X)=\beta_{j}\left(\frac{1+\beta_{1}}{\beta_{1}}\right)^{2} .
$$

Note that they are independent of $x_{r-1}, x_{r}$. 
LEMMA 7.6. The following relations hold:

$$
\begin{aligned}
& \alpha_{n} \beta_{n+1}-\alpha_{n+1} \beta_{n}=\beta_{1} \quad(0 \leq n \leq r-2) \\
& \Gamma_{n}^{(j)}(X) \Omega_{n}^{(j+1)}(X)+\Gamma_{n}^{(j+1)}(X) \Omega_{n}^{(j)}(X) \\
& -\Gamma_{n+1}^{(j)}(X) \Omega_{n-1}^{(j+1)}(X)-\Gamma_{n-1}^{(j+1)}(X) \Omega_{n+1}^{(j)}(X)=\left(\beta_{1}+1\right)^{2} \\
& \quad(0 \leq j \leq r-3,1 \leq n \leq r-2-j), \\
& \Pi_{n}^{(j)}(X) \Pi_{n}^{(j+1)}(X)-\Pi_{n+1}^{(j)}(X) \Pi_{n-1}^{(j+1)}(X)=-\beta_{1}\left(2+\beta_{1}\right) \\
& \quad(0 \leq j \leq r-3,1 \leq n \leq r-2-j) .
\end{aligned}
$$

Proof. It is easy to check (7.29). The rest are the consequences of Definition 7.5 and (7.29).

We define a family $\tau=\left\{\tau^{(a)}(u ; X) \in \mathbb{Z}\left[X^{ \pm 1}\right] \mid a \in I, u \in \mathbb{Z}, a+u\right.$ is odd/ even if $a \neq r / a=r\}$ as follows. First, we define, for $1 \leq a \leq r-2$ and $0 \leq$ $k \leq r-1$,

$$
\begin{aligned}
\tau^{(a)}(a-1+2 k ; X) \\
\quad= \begin{cases}\gamma_{k}^{(a+k)}(X) x_{a+k}+\nu_{k}^{(a+k)}(X) & (0 \leq k \leq r-a-2), \\
\Gamma_{r-a-1}^{(a+k-r+1)}(X) z+\Pi_{r-a-1}^{(a+k-r+1)}(X)+\frac{\Omega_{r-a-1}^{(a+k-r+1)}(X)}{z} & (r-a-1 \leq k \leq r-1),\end{cases}
\end{aligned}
$$

and, for $a=r-1, r$ and $0 \leq k \leq r-1$,

$$
\begin{gathered}
\tau^{(r-1)}(r-2+2 k ; X)= \begin{cases}\frac{\alpha_{k} z+\beta_{k}\left(1+\beta_{1}\right) / \beta_{1}}{x_{r}-1} & (k: \text { odd }), \\
\frac{\alpha_{k} z+\beta_{k}\left(1+\beta_{1}\right) / \beta_{1}}{x_{r}} & (k: \text { even }),\end{cases} \\
\tau^{(r)}(r-2+2 k ; X)= \begin{cases}\frac{\alpha_{k} z+\beta_{k}\left(1+\beta_{1}\right) / \beta_{1}}{x_{r}} & (k: \text { odd }), \\
\frac{\alpha_{k} z+\beta_{k}\left(1+\beta_{1}\right) / \beta_{1}}{x_{r-1}} & (k: \text { even }) .\end{cases}
\end{gathered}
$$

Then, we extend the definition by the following half-periodicity:

$$
\tau^{(a)}(u+2 r ; X)=\tau^{(\omega(a))}(u ; X),
$$

from which the periodicity $\tau^{(a)}(u+4 r ; X)=\tau^{(a)}(u ; X)$ also follows.

By (7.3), we have

$$
\tau^{(a)}(a-1 ; X)=x_{a} \quad(a=1, \ldots, r-1), \quad \tau^{(r)}(r-2 ; X)=x_{r} .
$$




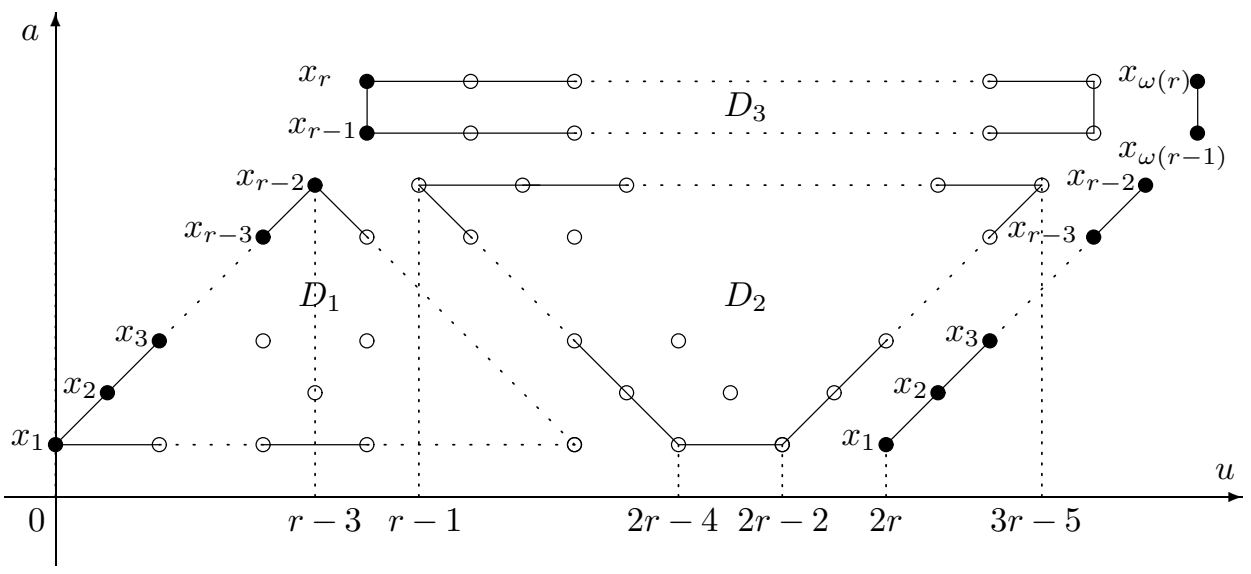

Figure 6: The half of the fundamental domain of $\tau^{(a)}(u ; X)$ for $\mathcal{T}_{2}\left(D_{r}\right)$

See Figure 6 for the half of the fundamental domain of $\tau^{(a)}(u ; X)$, which consists of three parts. The domains $D_{1}$ and $D_{2}$ correspond to the two cases of (7.32), while $D_{3}$ does the cases of (7.33) and (7.34):

$$
\begin{aligned}
& D_{1}=\{(a, a-1+2 k) \mid a=1, \ldots, r-2 ; 0 \leq k \leq r-a-2\}, \\
& D_{2}=\{(a, a-1+2 k) \mid a=1, \ldots, r-2 ; r-a-1 \leq k \leq r-1\}, \\
& D_{3}=\{(r-1, r-2+2 k),(r, r-2+2 k) \mid 0 \leq k \leq r-1\} .
\end{aligned}
$$

Using Lemmas 7.2 and 7.6, one can verify, case by case, the following.

Proposition 7.7. The family $\tau$ satisfies the T-system $\mathbb{T}_{2}\left(D_{r}\right)$ in $\mathbb{Z}\left[X^{ \pm 1}\right]$ :

$$
\tau^{(a)}(u-1 ; X) \tau^{(a)}(u+1 ; X)=1+\prod_{b \in I: C_{a b}=-1} \tau^{(b)}(u ; X) .
$$

Let $\mathcal{T}_{2}\left(D_{r}\right)_{+}$be the subring of $\mathcal{T}_{2}\left(D_{r}\right)$ generated by $T_{1}^{(a)}(u)^{ \pm 1}(a \in I$, $u \in \mathbb{Z}, a+u$ is odd/even if $a \neq r / a=r)$. Set

$$
T^{\text {init }}=\left\{T_{1}^{(a)}(a-1)(a=1, \ldots, r-1), T_{1}^{(r)}(r-2)\right\} .
$$

We call the elements of $T^{\text {init }}$ the initial variables of $\mathcal{T}_{2}\left(D_{r}\right)_{+}$.

THEOREM 7.8. The following relation holds in $\mathcal{T}_{2}\left(D_{r}\right)_{+}$:

$$
T_{1}^{(a)}(u)=\tau^{(a)}\left(u ; T^{\mathrm{init}}\right)
$$


where we set $x_{a}=T_{1}^{(a)}(a-1)(a \neq r)$ and $x_{r}=T_{1}^{(r)}(r-2)$ in the right-hand side.

As a corollary of Theorem 7.8 and (7.35), we once again obtain the periodicity of $\mathcal{T}_{2}\left(D_{r}\right)$ (Corollary 4.5 for $X_{r}=D_{r}$ ).

\subsection{Explicit formula by initial variables for $\mathcal{T}_{2}\left(B_{r}\right)$}

Again, the method is parallel to the former cases, so we present only the results for the most part. Throughout this subsection, $I=\{1, \ldots, r\}$ is the set enumerating the diagram $B_{r}$ in Figure 1. Recall that the dual Coxeter number of $B_{r}$ is $2 r-1$, and the number $t_{a}$ in (2.1) is 2 (resp. 1) if $a=r$ (otherwise).

For a family of variables $X=\left\{x_{a}, \bar{x}_{a}(a=1, \ldots, r-1), w_{1}, w_{2}, w_{3}\right\}$, we define $\gamma_{n}^{(j)}(X), \nu_{n}^{(j)}(X) \in \mathbb{Z}\left[X^{ \pm 1}\right](1 \leq j \leq r-1,0 \leq n \leq j)$ by Definition 7.1. We set

$$
\alpha_{n}=\gamma_{n}^{(r-1)}(X), \quad \beta_{n}=\nu_{n}^{(r-1)}(X), \quad z=w_{1} w_{3}
$$

Note that $\alpha_{r-1}=\beta_{0}=0$ and $\alpha_{0}=\beta_{r-1}=1$.

In the following, for $p(X) \in \mathbb{Z}\left[X^{ \pm 1}\right]$, we write $\bar{p}(X)$ for what obtained from $p(X)$ by replacing $x_{i}$ with $\bar{x}_{i}$. Similarly, we write $\widehat{p}(X)$ for what obtained from $p(X)$ by swapping $w_{1}$ and $w_{3}$.

Definition 7.9. We define $\mu_{n}(X), \delta_{n}(X) \in \mathbb{Z}\left[X^{ \pm 1}\right](0 \leq n \leq r)$ as

$$
\mu_{n}(X)=\frac{x_{n-1}}{x_{r-1}}, \quad \delta_{n}(X)= \begin{cases}\alpha_{n}+\frac{\beta_{n}}{x_{r-1}} & 0 \leq n \leq r-1, \\ 0 & n=r\end{cases}
$$

where we set $x_{0}=\bar{x}_{0}=1, x_{-1}=\bar{x}_{-1}=0$. In particular, we have $\mu_{0}(X)=0$, $\mu_{r}(X)=1, \delta_{0}(X)=1$, and $\delta_{r-1}(X)=1 / x_{r-1}$.

Note that $\mu_{n}(X)$ and $\delta_{n}(X)$ depend on $x_{r-1}$, while $\gamma_{n}^{(j)}(X)$ and $\nu_{n}^{(j)}(X)$ do not.

Definition 7.10. We define $P_{n}^{(j)}(X), Q_{n}^{(j)}(X), R_{n}^{(j)}(X), \eta_{n}(X), \xi_{n}(X) \in$ $\mathbb{Z}\left[X^{ \pm 1}\right]$ as follows. For $0 \leq j \leq r, 0 \leq k \leq r, 1 \leq j+k \leq r+1$,

$$
\begin{aligned}
& P_{2 k}^{(j)}(X)=\frac{x_{k-1}}{z} \bar{\delta}_{j-1+k}(X), \\
& Q_{2 k}^{(j)}(X)=x_{k-1} \bar{\mu}_{j+k-1}+\bar{\delta}_{j+k-1}(X) \bar{x}_{r-1}\left(\delta_{k}(X)+\frac{2 x_{k-1}}{z}\right), \\
& R_{2 k}^{(j)}(X)=\bar{\delta}_{j-1+k}(X) x_{k-1} \bar{x}_{r-1}\left(\frac{\bar{x}_{r-1}}{z}+\frac{1}{x_{r-1}}\right) .
\end{aligned}
$$


For $0 \leq j \leq r, 0 \leq k \leq r, 0 \leq j+k \leq r$,

$$
\begin{aligned}
& P_{2 k+1}^{(j)}(X)=\bar{\delta}_{k}(X) \delta_{j+k}(X) \\
& Q_{2 k+1}^{(j)}(X)=z \delta_{j+k}(X) \bar{\mu}_{k}(X)+\bar{\delta}_{k}(X) \mu_{j+k}(X), \\
& R_{2 k+1}^{(j)}(X)=z x_{j-1+k} \bar{\mu}_{k}(X)\left(\frac{\bar{x}_{r-1}}{z}+\frac{1}{x_{r-1}}\right) .
\end{aligned}
$$

And,

$$
\begin{aligned}
& \eta_{2 k}(X)=w_{1} \delta_{k}(X)+\frac{x_{k-1}}{w_{3}}, \\
& \xi_{2 k}(X)=w_{1} x_{k-1}\left(\frac{\bar{x}_{r-1}}{z}+\frac{1}{x_{r-1}}\right) \quad(0 \leq k \leq r),
\end{aligned}
$$

$$
\begin{aligned}
& \eta_{2 k+1}(X)=\frac{\bar{\delta}_{k}(X)}{w_{1}}, \\
& \xi_{2 k+1}(X)=\frac{1}{w_{1}}\left(\bar{x}_{r-1} \bar{\delta}_{k}(X)+z \bar{\mu}_{k}(X)\right) \quad(0 \leq k \leq r-1) .
\end{aligned}
$$

In particular, we have $Q_{1}^{(0)}(X)=R_{1}^{(0)}(X)=P_{2 r+1}^{(0)}(X)=Q_{2 r+1}^{(0)}(X)=0$ and $P_{1}^{(0)}(X)=1$.

LEMma 7.11. The following relations hold. For $1 \leq k \leq r$,

$$
\begin{aligned}
& \mu_{k-1}(X) x_{k-1}-\mu_{k}(X) x_{k-2}=\bar{\mu}_{k-1}(X) \bar{x}_{k-1}-\bar{\mu}_{k}(X) \bar{x}_{k-2}=0, \\
& \delta_{k-1}(X) x_{k-1}-\delta_{k}(X) x_{k-2}=\bar{\delta}_{k-1}(X) \bar{x}_{k-1}-\bar{\delta}_{k}(X) \bar{x}_{k-2}=1 .
\end{aligned}
$$

For $1 \leq j \leq r-1,0 \leq k \leq 2 r-2,1 \leq j+[k / 2] \leq r$,

$$
\begin{aligned}
& Q_{k}^{(j)}(X) R_{k+2}^{(j)}(X)+Q_{k+2}^{(j)}(X) R_{k}^{(j)}(X) \\
& \quad-Q_{k+2}^{(j-1)}(X) R_{k}^{(j+1)}(X)-Q_{k}^{(j+1)}(X) R_{k+2}^{(j-1)}(X)=0, \\
& Q_{k}^{(j)}(X) P_{k+2}^{(j)}(X)+Q_{k+2}^{(j)}(X) P_{k}^{(j)}(X) \\
& \quad-Q_{k+2}^{(j-1)}(X) P_{k}^{(j+1)}(X)-Q_{k}^{(j+1)}(X) P_{k+2}^{(j-1)}(X)=0, \\
& Q_{k}^{(j)}(X) Q_{k+2}^{(j)}(X)-Q_{k+2}^{(j-1)}(X) Q_{k}^{(j+1)}(X) \\
& \quad= \begin{cases}-z \mu_{1}(X) \bar{\mu}_{1}(X) & k: \text { odd }, \\
1 & k: \text { even, }\end{cases}
\end{aligned}
$$




$$
\begin{array}{r}
P_{k}^{(j)}(X) R_{k+2}^{(j)}(X)+P_{k+2}^{(j)}(X) R_{k}^{(j)}(X)-P_{k+2}^{(j-1)}(X) R_{k}^{(j+1)}(X) \\
-P_{k}^{(j+1)}(X) R_{k+2}^{(j-1)}(X)= \begin{cases}1+z \mu_{1}(X) \bar{\mu}_{1}(X) & k: \text { odd }, \\
0 & k: \text { even } .\end{cases}
\end{array}
$$

For $1 \leq k \leq 2 r$,

$$
\begin{aligned}
Q_{k}^{(0)}( & X) R_{k+1}^{(0)}(X)+Q_{k+1}^{(0)}(X) R_{k}^{(0)}(X) \\
& -Q_{k}^{(1)}(X) R_{k-1}^{(1)}(X)-Q_{k-1}^{(1)}(X) R_{k}^{(1)}(X) \\
= & \begin{cases}0 & k: \text { odd }, \\
\xi_{k}(X) \widehat{\eta}_{k}(X)+\widehat{\xi}_{k}(X) \eta_{k}(X) & k: \text { even },\end{cases} \\
Q_{k}^{(0)}( & X) P_{k+1}^{(0)}(X)+Q_{k+1}^{(0)}(X) P_{k}^{(0)}(X) \\
& -Q_{k}^{(1)}(X) P_{k-1}^{(1)}(X)-Q_{k-1}^{(1)}(X) P_{k}^{(1)}(X) \\
= & \begin{cases}\xi_{k}(X) \widehat{\eta}_{k}(X)+\widehat{\xi}_{k}(X) \eta_{k}(X) & k: \text { odd, } \\
0 & k: \text { even, }\end{cases} \\
P_{k}^{(0)}( & X) R_{k+1}^{(0)}(X)+P_{k+1}^{(0)}(X) R_{k}^{(0)}(X)-P_{k}^{(1)}(X) R_{k-1}^{(1)}(X) \\
- & P_{k-1}^{(1)}(X) R_{k}^{(1)}(X)+Q_{k}^{(0)}(X) Q_{k+1}^{(0)}(X)-Q_{k+1}^{(1)}(X) Q_{k}^{(1)}(X) \\
= & \begin{cases}\xi_{k}(X) \widehat{\xi}_{k}(X) & k: \text { odd }, \\
\eta_{k}(X) \widehat{\eta}_{k}(X) & k: \text { even. }\end{cases}
\end{aligned}
$$

Proof. It is easy to check (7.46). The rest are the consequence of Definitions $7.9,7.10$, and (7.46).

For a triplet $(a, m, u)\left(a \in I ; m=1, \ldots, 2 t_{a}-1 ; u \in \frac{1}{2} \mathbb{Z}\right)$, we set the condition,

$$
\text { Condition }(\mathrm{P}): \quad u \in \begin{cases}\mathbb{Z}+\frac{1}{2} & (a, m)=(r, 1),(r, 3), \\ \mathbb{Z} & \text { otherwise. }\end{cases}
$$

We define a family $\tau=\left\{\tau_{m}^{(a)}(u ; X) \in \mathbb{Z}\left[X^{ \pm 1}\right] \mid a \in I ; m=1, \ldots, 2 t_{a}-1 ; u \in\right.$ $(1 / 2) \mathbb{Z}$; Condition $(\mathrm{P})\}$ as follows. First, we define $\tau_{m}^{(a)}(u ; X)$ in the following 
region:

$$
\begin{aligned}
& \left\{\begin{array}{c}
\tau_{1}^{(a)}(a-1+2 k ; X)=\gamma_{k}^{(a+k)}(X) x_{a+k}+\nu_{k}^{(a+k)}(X) \\
(1 \leq a \leq r-1,0 \leq k \leq r-1-a) \\
\tau_{1}^{(a)}(a+2 k ; X)=\bar{\tau}_{1}^{(a)}(a-1+2 k ; X) \\
(1 \leq a \leq r-1,0 \leq k \leq r-1-a)
\end{array}\right. \\
& \left\{\begin{array}{c}
\tau_{1}^{(a)}(a+2(r-1-a)+k ; X) \\
=P_{k}^{(r-a)}(X) w_{2}+Q_{k}^{(r-a)}(X)+\frac{R_{k}^{(r-a)}(X)}{w_{2}} \\
\quad(1 \leq a \leq r-1,1 \leq k \leq 2 a+1), \\
\tau_{2}^{(r)}(r-1+k ; X)=P_{k+1}^{(0)}(X) w_{2}+Q_{k+1}^{(0)}(X)+\frac{R_{k+1}^{(0)}(X)}{w_{2}} \\
(0 \leq k \leq 2 r),
\end{array}\right. \\
& \begin{cases}\tau_{1}^{(r)}\left(r+2 k-\frac{1}{2} ; X\right)=\eta_{2 k+1}(X) w_{2}+\xi_{2 k+1}(X) & (0 \leq k \leq r-1), \\
\tau_{1}^{(r)}\left(r+2 k-\frac{3}{2} ; X\right)=\eta_{2 k}(X)+\frac{\xi_{2 k}(X)}{w_{2}} & (0 \leq k \leq r) \\
\tau_{3}^{(r)}\left(r+k-\frac{3}{2} ; X\right)=\widehat{\tau}_{1}^{(r)}\left(r+k-\frac{3}{2} ; X\right) & (0 \leq k \leq 2 r) .\end{cases}
\end{aligned}
$$

Then, we extend the definition by the following half-periodicity:

$$
\tau_{m}^{(a)}(u+2 r+1 ; X)=\widehat{\tau}_{m}^{(a)}(u ; X)
$$

from which the periodicity $\tau_{m}^{(a)}(u+2(2 r+1) ; X)=\tau_{m}^{(a)}(u ; X)$ also follows.

By (7.3), we have

$$
\begin{array}{rlrl}
\tau_{1}^{(a)}(a-1 ; X) & =x_{a}, & \tau_{1}^{(a)}(a ; X)=\bar{x}_{a} \quad(a=1, \ldots, r-1), \\
\tau_{1}^{(r)}\left(r-\frac{3}{2} ; X\right) & =w_{1}, \quad \tau_{2}^{(r)}(r-1 ; X)=w_{2}, \\
\tau_{3}^{(r)}\left(r-\frac{3}{2} ; X\right) & =w_{3} .
\end{array}
$$

See Figure 7 for the half of the fundamental domain of $\tau_{m}^{(a)}(u ; X)$ which consists of three parts. The domains $D_{1}, D_{2}$, and $D_{3}$ correspond to the cases $(7.50),(7.51)$, and $(7.52)$, respectively:

$$
\begin{aligned}
D_{1}= & \{(a, a-1+k) \mid 1 \leq a \leq r-1 ; 0 \leq k \leq 2(r-a)-1\}, \\
D_{2}= & \{(a, a-1+k) \mid 1 \leq a \leq r-1 ; 2(r-a) \leq k \leq 2 r\} \\
& \sqcup\left\{\left(r_{2}, r-1+k\right) \mid 0 \leq k \leq 2 r\right\}, \\
D_{3}= & \left\{\left(r_{1}, r+k-\frac{3}{2}\right),\left(r_{3}, r+k-\frac{3}{2}\right) \mid 0 \leq k \leq 2 r\right\},
\end{aligned}
$$




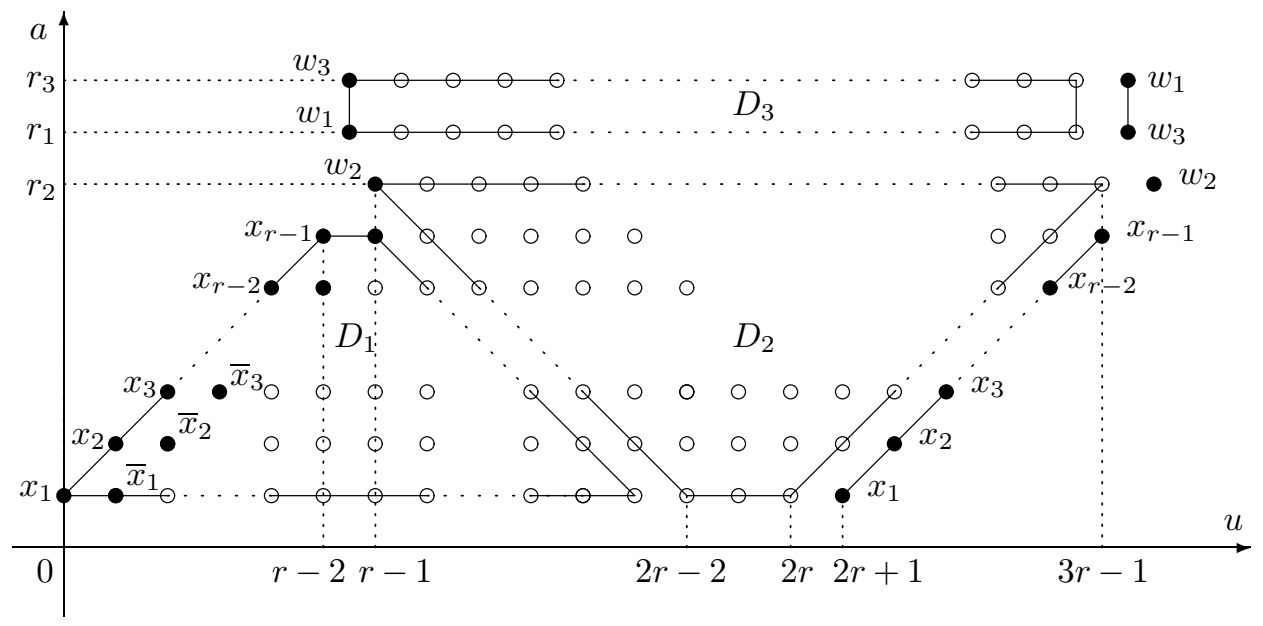

Figure 7: The half of the fundamental domain of $\tau_{m}^{(a)}(u ; X)$ for $\mathcal{T}_{2}\left(B_{r}\right)$

where the point $\left(r_{i}, u\right)$ corresponds to $\tau_{i}^{(r)}(u)$.

Using Lemmas 7.2 and 7.11, one can verify the following, case by case.

Proposition 7.12. The family $\tau$ satisfies the $T$-system $\mathbb{T}_{2}\left(B_{r}\right)$ in $\mathbb{Z}\left[X^{ \pm 1}\right]$ (by replacing $T_{m}^{(a)}(u)$ in $\mathbb{T}_{2}\left(B_{r}\right)$ with $\tau_{m}^{(a)}(u ; X)$ ).

Let $\mathcal{T}_{2}\left(B_{r}\right)_{+}$be the subring of $\mathcal{T}_{2}\left(B_{r}\right)$ generated by $T_{m}^{(a)}(u)^{ \pm 1}(a \in I ; m=$ $1, \ldots, 2 t_{a}-1 ; u \in(1 / 2) \mathbb{Z}$; Condition $\left.(\mathrm{P})\right)$. Set

$$
\begin{aligned}
T^{\text {init }}= & \left\{T_{1}^{(a)}(a-1), T_{1}^{(a)}(a)(a=1, \ldots, r-1),\right. \\
& \left.T_{1}^{(r)}\left(r-\frac{3}{2}\right), T_{2}^{(r)}(r-1), T_{3}^{(r)}\left(r-\frac{3}{2}\right)\right\} .
\end{aligned}
$$

We call the elements of $T^{\text {init }}$ the initial variables of $\mathcal{T}_{2}\left(B_{r}\right)_{+}$.

THEOREM 7.13. The following relation holds in $\mathcal{T}_{2}\left(B_{r}\right)_{+}$:

$$
T_{m}^{(a)}(u)=\tau_{m}^{(a)}\left(u ; T^{\mathrm{init}}\right)
$$

where we set $x_{a}=T_{1}^{(a)}(a-1), \bar{x}_{a}=T_{1}^{(a)}(a) \quad(a \neq r), w_{1}=T_{1}^{(r)}(r-(3 / 2))$, $w_{2}=T_{2}^{(r)}(r-1)$, and $w_{3}=T_{3}^{(r)}(r-(3 / 2))$ in the right-hand side.

As a corollary of Theorem 7.13 and (7.53), we obtain the following. 
COROLlary 7.14. The following relations hold in $\mathcal{T}_{2}\left(B_{r}\right)$ :

(1) Half-periodicity: $T_{m}^{(a)}(u+2 r+1)=T_{2 t_{a}-m}^{(a)}(u)$.

(2) Periodicity: $T_{m}^{(a)}(u+2(2 r+1))=T_{m}^{(a)}(u)$.

\section{§8. Periodicities of restricted T- and $\mathrm{Y}$-systems at levels 1 and 0}

So far, we have assumed that the level $\ell$ for the restriction is greater than or equal to 2 . In this section we extend the periodicity property of the restricted $\mathrm{T}$ - and Y-systems at levels 1 and 0.

\subsection{Periodicities of restricted $\mathrm{T}$ - and $\mathrm{Y}$-systems at level $\mathbf{1}$}

In the systems $\mathbb{T}_{\ell}\left(X_{r}\right)$ and $\mathbb{Y}_{\ell}\left(X_{r}\right)$, we treat the variables $T_{m}^{(a)}(u)$ and $Y_{m}^{(a)}(u)$ with $m=1, \ldots, t_{a} \ell-1$. Thus at $\ell=1$, these systems are void for simply laced $X_{r}$; however, Definitions $3.1,3.2,3.5$, and 3.6 still make sense for nonsimply laced $X_{r}$.

The level $1 \mathrm{~T}$ - and Y-systems in these cases are actually equivalent to the systems of type $A$. To illustrate, consider $\mathbb{T}_{1}\left(F_{4}\right)$ :

$$
\begin{aligned}
& T_{1}^{(3)}\left(u-\frac{1}{2}\right) T_{1}^{(3)}\left(u+\frac{1}{2}\right)=1+T_{1}^{(4)}(u), \\
& T_{1}^{(4)}\left(u-\frac{1}{2}\right) T_{1}^{(4)}\left(u+\frac{1}{2}\right)=1+T_{1}^{(3)}(u),
\end{aligned}
$$

where we have omitted the first three relations in $(2.5)$, which are void at $\ell=1$. To be precise, let us introduce another level $\ell$ restricted T-system $\mathbb{T}_{\ell}^{\prime}\left(A_{r}\right)$ for $T=\left\{T_{m}^{(a)}(u) \mid a=1, \ldots, r ; m=1, \ldots, \ell-1 ; u \in(1 / \ell) \mathbb{Z}\right\}$ with the relations

$$
T_{m}^{(a)}\left(u-\frac{1}{\ell}\right) T_{m}^{(a)}\left(u+\frac{1}{\ell}\right)=T_{m-1}^{(a)}(u) T_{m+1}^{(a)}(u)+T_{m}^{(a-1)}(u) T_{m}^{(a+1)}(u)
$$

where the left-hand side of (8.2) differs from (2.2) for $\mathbb{T}_{\ell}\left(A_{r}\right)$. Then, the relations in (8.1) are equivalent to $\mathbb{T}_{2}^{\prime}\left(A_{2}\right)$. In other words, $\mathcal{T}_{1}\left(F_{4}\right) \simeq \mathcal{T}_{2}^{\prime}\left(A_{2}\right)$, where $\mathcal{T}_{\ell}^{\prime}\left(A_{r}\right)$ denotes the T-algebra associated with $\mathbb{T}_{\ell}^{\prime}\left(A_{r}\right)$. A similar reduction of the Y-system, $y_{1}\left(F_{4}\right) \simeq y_{2}^{\prime}\left(A_{2}\right)$, happens, where $\mathbb{Y}_{\ell}^{\prime}\left(A_{r}\right)$ and $y_{\ell}^{\prime}\left(A_{r}\right)$ are defined in the same way.

In general, $\mathcal{T}_{1}\left(X_{r}\right) \simeq \mathcal{T}_{t}^{\prime}\left(A_{r^{\prime}}\right)$ and $y_{1}\left(X_{r}\right) \simeq y_{t}^{\prime}\left(A_{r^{\prime}}\right)$ hold for nonsimply laced $X_{r}$, where $t$ is the number in (2.1) and $r^{\prime}$ equals the number of the short simple roots of $X_{r}$. 
Let us summarize the relevant data for the periodicities of $\mathcal{T}_{1}\left(X_{r}\right)$ and $y_{1}\left(X_{r}\right)$ :

\begin{tabular}{c||c|c|c|c}
$X_{r}$ & $A_{r^{\prime}}$ & $t$ & $h^{\vee}+1$ & $\left(r^{\prime}+1+t\right) / t$ \\
\hline$B_{r}$ & $A_{1}$ & 2 & $2 r$ & 2 \\
$C_{r}$ & $A_{r-1}$ & 2 & $r+2$ & $(r+2) / 2$ \\
$F_{4}$ & $A_{2}$ & 2 & 10 & $5 / 2$ \\
$G_{2}$ & $A_{1}$ & 3 & 5 & $5 / 3$
\end{tabular}

THEOREM 8.1. (i) For any nonsimply laced $X_{r}$, the following relations hold in $\mathcal{T}_{1}\left(X_{r}\right)$ :

(1) Half-periodicity: $T_{m}^{(a)}\left(u+h^{\vee}+1\right)=T_{t_{a}-m}^{(a)}(u)$.

(2) Periodicity: $T_{m}^{(a)}\left(u+2\left(h^{\vee}+1\right)\right)=T_{m}^{(a)}(u)$.

(ii) For any nonsimply laced $X_{r}$, the following relations hold in $y_{1}\left(X_{r}\right)$ :

(1) Half-periodicity: $Y_{m}^{(a)}\left(u+h^{\vee}+1\right)=Y_{t_{a}-m}^{(a)}(u)$.

(2) Periodicity: $Y_{m}^{(a)}\left(u+2\left(h^{\vee}+1\right)\right)=Y_{m}^{(a)}(u)$.

Proof. By inspecting (8.3), one can check that the half-periodicities of $\mathcal{T}_{1}\left(X_{r}\right)$ and $y_{1}\left(X_{r}\right)$ follow from the half- or full-periodicities of the corresponding $\mathcal{T}_{t}^{\prime}\left(A_{r^{\prime}}\right)$ and $y_{t}^{\prime}\left(A_{r^{\prime}}\right)$.

\subsection{Periodicities of restricted $\mathbf{T}$ - and $\mathrm{Y}$-systems at level 0}

At level 0, one can still introduce, at least formally, a restricted $\mathrm{T}$ - and Y-system for any $X_{r}$, and study their periodicity.

Definition 8.2. The level 0 restricted T-system $\mathbb{T}_{0}\left(X_{r}\right)$ of type $X_{r}$ is the following system of relations for a family of variables $T=\left\{T^{(a)}(u) \mid a \in\right.$ $I, u \in U\}$, where $T^{(0)}(u)=1$ if they occur in the right-hand sides in the relations.

For simply laced $X_{r}$,

$$
T^{(a)}(u-1) T^{(a)}(u+1)=\prod_{b \in I: C_{a b}=-1} T^{(b)}(u) .
$$

For $X_{r}=B_{r}$

$$
\begin{aligned}
T^{(a)}(u-1) T^{(a)}(u+1) & =T^{(a-1)}(u) T^{(a+1)}(u) \quad(1 \leq a \leq r-2), \\
T^{(r-1)}(u-1) T^{(r-1)}(u+1) & =T^{(r-2)}(u) T^{(r)}(u) \\
T^{(r)}\left(u-\frac{1}{2}\right) T^{(r)}\left(u+\frac{1}{2}\right) & =T^{(r-1)}\left(u-\frac{1}{2}\right) T^{(r-1)}\left(u+\frac{1}{2}\right) .
\end{aligned}
$$


For $X_{r}=C_{r}$,

$$
\begin{aligned}
T^{(a)}\left(u-\frac{1}{2}\right) T^{(a)}\left(u+\frac{1}{2}\right) & =T^{(a-1)}(u) T^{(a+1)}(u) \quad(1 \leq a \leq r-2), \\
T^{(r-1)}\left(u-\frac{1}{2}\right) T^{(r-1)}\left(u+\frac{1}{2}\right) & =T^{(r-2)}(u) T^{(r)}\left(u-\frac{1}{2}\right) T^{(r)}\left(u+\frac{1}{2}\right), \\
T^{(r)}(u-1) T^{(r)}(u+1) & =T^{(r-1)}(u) .
\end{aligned}
$$

For $X_{r}=F_{4}$,

$$
\begin{aligned}
T^{(1)}(u-1) T^{(1)}(u+1) & =T^{(2)}(u), \\
T^{(2)}(u-1) T^{(2)}(u+1) & =T^{(1)}(u) T^{(3)}(u), \\
T^{(3)}\left(u-\frac{1}{2}\right) T^{(3)}\left(u+\frac{1}{2}\right) & =T^{(2)}\left(u-\frac{1}{2}\right) T^{(2)}\left(u+\frac{1}{2}\right) T^{(4)}(u), \\
T^{(4)}\left(u-\frac{1}{2}\right) T^{(4)}\left(u+\frac{1}{2}\right) & =T^{(3)}(u) .
\end{aligned}
$$

For $X_{r}=G_{2}$,

$$
\begin{aligned}
T^{(1)}(u-1) T^{(1)}(u+1) & =T^{(2)}(u), \\
T^{(2)}\left(u-\frac{1}{3}\right) T^{(2)}\left(u+\frac{1}{3}\right) & =T^{(1)}\left(u-\frac{2}{3}\right) T^{(1)}(u) T^{(1)}\left(u+\frac{2}{3}\right) .
\end{aligned}
$$

Definition 8.3. The level 0 restricted T-group $\mathcal{T}_{0}\left(X_{r}\right)$ of type $X_{r}$ is the abelian group with generators $T^{(a)}(u)(a \in I, u \in U)$ and the relations $\mathbb{T}_{0}\left(X_{r}\right)$.

REMARK 8.4. $\mathbb{T}_{0}\left(X_{r}\right)$ is obtained from the unrestricted T-system $\mathbb{T}\left(X_{r}\right)$ (2.2)-(2.6) by setting $T_{m}^{(a)}(u)=T^{(a)}(u)$ if $m=0$ and $T_{m}^{(a)}(u)=0$ otherwise. It was originally introduced in [KNS2, Section 2.2] as "bulk T-system."

Similarly, we define the following.

Definition 8.5. The level 0 restricted $Y$-system $\mathbb{Y}_{0}\left(X_{r}\right)$ of type $X_{r}$ is the following system of relations for a family of variables $Y=\left\{Y^{(a)}(u) \mid a \in\right.$ $I, u \in U\}$, where $Y^{(0)}(u)=1$ if they occur in the right-hand sides in the relations.

For simply laced $X_{r}$,

$$
Y^{(a)}(u-1) Y^{(a)}(u+1)=\prod_{b \in I: C_{a b}=-1} Y^{(b)}(u) .
$$


For $X_{r}=B_{r}$,

$$
\begin{aligned}
Y^{(a)}(u-1) Y^{(a)}(u+1) & =Y^{(a-1)}(u) Y^{(a+1)}(u) \quad(1 \leq a \leq r-2), \\
Y^{(r-1)}(u-1) Y^{(r-1)}(u+1) & =Y^{(r-2)}(u) Y^{(r)}\left(u-\frac{1}{2}\right) Y^{(r)}\left(u+\frac{1}{2}\right) \\
Y^{(r)}\left(u-\frac{1}{2}\right) Y^{(r)}\left(u+\frac{1}{2}\right) & =Y^{(r-1)}(u) .
\end{aligned}
$$

For $X_{r}=C_{r}$,

$$
\begin{aligned}
Y^{(a)}\left(u-\frac{1}{2}\right) Y^{(a)}\left(u+\frac{1}{2}\right) & =Y^{(a-1)}(u) Y^{(a+1)}(u) \quad(1 \leq a \leq r-2), \\
Y^{(r-1)}\left(u-\frac{1}{2}\right) Y^{(r-1)}\left(u+\frac{1}{2}\right) & =Y^{(r-2)}(u) Y^{(r)}(u), \\
Y^{(r)}(u-1) Y^{(r)}(u+1) & =Y^{(r-1)}\left(u-\frac{1}{2}\right) Y^{(r-1)}\left(u+\frac{1}{2}\right) .
\end{aligned}
$$

For $X_{r}=F_{4}$,

$$
\begin{aligned}
Y^{(1)}(u-1) Y^{(1)}(u+1) & =Y^{(2)}(u), \\
Y^{(2)}(u-1) Y^{(2)}(u+1) & =Y^{(1)}(u) Y^{(3)}\left(u-\frac{1}{2}\right) Y^{(3)}\left(u+\frac{1}{2}\right), \\
Y^{(3)}\left(u-\frac{1}{2}\right) Y^{(3)}\left(u+\frac{1}{2}\right) & =Y^{(2)}(u) Y^{(4)}(u), \\
Y^{(4)}\left(u-\frac{1}{2}\right) Y^{(4)}\left(u+\frac{1}{2}\right) & =Y^{(3)}(u) .
\end{aligned}
$$

For $X_{r}=G_{2}$,

$$
\begin{aligned}
Y^{(1)}(u-1) Y^{(1)}(u+1) & =Y^{(2)}\left(u-\frac{2}{3}\right) Y^{(2)}(u) Y^{(2)}\left(u+\frac{2}{3}\right), \\
Y^{(2)}\left(u-\frac{1}{3}\right) Y^{(2)}\left(u+\frac{1}{3}\right) & =Y^{(1)}(u) .
\end{aligned}
$$

Definition 8.6. The level 0 restricted $Y$-group $y_{0}\left(X_{r}\right)$ of type $X_{r}$ is the abelian group with generators $Y^{(a)}(u)(a \in I, u \in U)$ and the relations $\mathbb{Y}_{0}\left(X_{r}\right)$.

REMARK 8.7. $\mathbb{Y}_{0}\left(X_{r}\right)$ is obtained from unrestricted Y-system $\mathbb{Y}\left(X_{r}\right)$ (2.15)-(2.19) by first making the replacement $\left(1+Y_{m}^{(a)}(u), 1+Y_{m}^{(a)}(u)^{-1}\right) \rightarrow$ $\left(Y_{m}^{(a)}(u), 1\right)$ in the right-hand sides (i.e., taking a formal limit $\left.Y_{m}^{(a)}(u) \rightarrow \infty\right)$, and then setting $Y_{m}^{(a)}(u)=Y^{(a)}(u)$ if $m=0$ and $Y_{m}^{(a)}(u)=1$ otherwise. 
From (8.4) and (8.9), we see that $\mathcal{T}_{0}\left(X_{r}\right) \simeq \mathfrak{y}_{0}\left(X_{r}\right)$ if $X_{r}$ is simply laced. The following periodicity property justifies that we call these systems "level 0." Notice that the half-periodicity here contains the inverse in the right-hand sides, in contrast with the level $\ell \geq 1$ case.

THEOREM 8.8. (i) The following relations hold in $\mathcal{T}_{0}\left(X_{r}\right)$.

(1) Half-periodicity: $T^{(a)}\left(u+h^{\vee}\right)=T^{(\omega(a))}(u)^{-1}$.

(2) Periodicity: $T^{(a)}\left(u+2 h^{\vee}\right)=T^{(a)}(u)$.

(ii) The following relations hold in $y_{0}\left(X_{r}\right)$.

(1) Half-periodicity: $Y^{(a)}\left(u+h^{\vee}\right)=Y^{(\omega(a))}(u)^{-1}$.

(2) Periodicity: $Y^{(a)}\left(u+2 h^{\vee}\right)=Y^{(a)}(u)$.

Proof. It is enough to show the half-periodicity, and it can be proved by elementary manipulations. Especially for exceptional $X_{r}=E_{6}, E_{7}, E_{8}, F_{4}$, and $G_{2}$, it is a matter of a direct check. As an illustration we present a proof for $\mathcal{T}_{0}\left(D_{r}\right)\left(\simeq y_{0}\left(D_{r}\right)\right)$ and $y_{0}\left(B_{r}\right)$ below. The cases $\mathcal{T}_{0}\left(B_{r}\right), \mathcal{T}_{0}\left(C_{r}\right), \mathcal{T}_{0}\left(F_{4}\right)$, and $\mathcal{T}_{0}\left(G_{2}\right)$ have been treated in [KNS2, Section 2.2.1 and Appendix A].

First we consider $\mathcal{T}_{0}\left(D_{r}\right)$. From (8.4) we have

$$
\begin{aligned}
T^{(a)}(u) & =\prod_{s=1}^{a} T^{(1)}(u-a-1+2 s) \quad(1 \leq a \leq r-2), \\
T^{(r-1)}(u) T^{(r)}(u) & =\prod_{s=1}^{r-1} T^{(1)}(u-r+2 s) \\
T^{(a)}(u-1) T^{(a)}(u+1) & =T^{(r-2)}(u) \quad(a=r-1, r) .
\end{aligned}
$$

In (8.15) replace $u$ by $u \pm 1$ and take the product. Using (8.14) with $a=$ $r-2$ and (8.16), one can express all the factors by $T^{(1)}$ only, leading to $T^{(1)}(u-r+1) T^{(1)}(u+r-1)=1$. In view of $h^{\vee}=2 r-2$ and (8.14), this verifies the claim of the theorem $T^{(a)}(u) T^{(a)}\left(u+h^{\vee}\right)=1$ except for $a=r-1$ and $r$.

Suppose $r$ is even. Then for $a=r-1, r$ we have

$$
T^{(a)}(u) T^{(a)}\left(u+h^{\vee}\right)=\frac{\prod_{s=0}^{r-1} T^{(a)}(u+2 s)}{\prod_{s=1}^{r-2} T^{(a)}(u+2 s)}=\frac{\prod_{s=0}^{r / 2-1} T^{(r-2)}(u+1+4 s)}{\prod_{s=1}^{r / 2-1} T^{(r-2)}(u-1+4 s)},
$$

where the second equality is due to (8.16). From (8.14) with $a=r-2$, the ratio is expressed by $T^{(1)}$ only, which turns out to be 1 owing to 
$T^{(1)}(u) T^{(1)}\left(u+h^{\vee}\right)=1$. If $r$ is odd, let $\bar{r}=r-1$ and $\overline{r-1}=r$. Then we have

$$
\begin{aligned}
T^{(a)}(u) T^{(\bar{a})}\left(u+h^{\vee}\right)= & \frac{\left(\prod_{s=0}^{r-1} T^{(a)}(u+2 s)\right) T^{(\bar{a})}\left(u+h^{\vee}\right)}{\prod_{s=1}^{r-1} T^{(a)}(u+2 s)} \\
= & \frac{\prod_{s=0}^{(r-3) / 2} T^{(r-2)}(u+1+4 s)}{\prod_{s=1}^{(r-1) / 2} T^{(r-2)}(u-1+4 s)} \\
& \times T^{(r-1)}\left(u+h^{\vee}\right) T^{(r)}\left(u+h^{\vee}\right),
\end{aligned}
$$

where the second equality is due to (8.16). Again, this can be shown to be 1 from (8.14) with $a=r-2,(8.15)$, and $T^{(1)}(u) T^{(1)}\left(u+h^{\vee}\right)=1$.

Next we consider $y_{0}\left(B_{r}\right)$. From (8.10) we find

$$
Y^{(a)}(u)=\prod_{s=1}^{a} Y^{(1)}(u-a-1+2 s)
$$

$$
(1 \leq a \leq r-1)
$$

$$
\begin{aligned}
Y^{(r-1)}(u-1) Y^{(r-1)}(u+1) & =Y^{(r-2)}(u) Y^{(r-1)}(u), \\
Y^{(r)}\left(u-\frac{1}{2}\right) Y^{(r)}\left(u+\frac{1}{2}\right) & =Y^{(r-1)}(u) .
\end{aligned}
$$

Substituting (8.17) into (8.18), we get $Y^{(r-1)}(u)=\prod_{s=1}^{r} Y^{(1)}(u-r-1+2 s)$. Comparing this with expression (8.17) with $a=r-1$, we have

$$
Y^{(r-1)}(u)=Y^{(r-1)}(u \pm 1) Y^{(1)}(u \mp r \pm 1) .
$$

The two relations imply $Y^{(1)}(u-r+1) Y^{(1)}(u+r)=1$. In view of $h^{\vee}=2 r-1$ and (8.17), this verifies the claim of the theorem $Y^{(a)}(u) Y^{(a)}\left(u+h^{\vee}\right)=1$ except for $a=r$. From either of the relations (8.20) and (8.17) with $a=$ $r-1$, one can derive $\prod_{s=1}^{r} Y^{(r-1)}(u-r-1+2 s)=\prod_{s=1}^{r-1} Y^{(r-1)}(u-r+2 s)$. Substitution of (8.19) into this gives $Y^{(r)}(u) Y^{(r)}\left(u+h^{\vee}\right)=1$.

For simply laced $X_{r}$, a more intrinsic proof of Theorem 8.8 by the Coxeter element of the Weyl group is available, following the remarkable idea by Fomin and Zelevinsky [FZ3] used for the proof of the periodicity of $y_{2}\left(X_{r}\right)$.

Alternative proof for simply laced $X_{r}$. Assume that $X_{r}$ is simply laced. Let $I=I_{+} \sqcup I_{-}$be the bipartite decomposition of the index set $I$, and define $\varepsilon(a)$ by $\varepsilon(a)= \pm$ for $a \in I_{ \pm}$. Since $\mathbb{T}_{0}\left(X_{r}\right)$ closes among those $T^{(a)}(u)$ 
with fixed "parity" $\varepsilon(a)(-1)^{u}$, it is no problem to impose an additional relation in $\mathcal{T}_{0}\left(X_{r}\right)$,

$$
T^{(a)}(u+1)=T^{(a)}(u)^{-1} \quad \text { whenever } \varepsilon(a)(-1)^{u}=+
$$

in order to prove its periodicity. Let $W$ be the Weyl group of type $X_{r}$ with the simple reflections $s_{a}(a \in I)$, which acts on $\mathcal{T}_{0}\left(X_{r}\right)$ by

$$
s_{b}\left(T^{(a)}(u)\right)=T^{(a)}(u) T^{(b)}(u)^{-C_{b a}} .
$$

Define $\tau_{ \pm}=\prod_{a \in I_{ \pm}} s_{a}$. Then, $\tau_{ \pm} \tau_{\mp}$ is the Coxeter element of $W$, and $\tau_{\varepsilon}$ acts as

$$
\tau_{\varepsilon}\left(T^{(a)}(u)\right)= \begin{cases}T^{(a)}(u)^{-1} & \varepsilon(a)=\varepsilon \\ T^{(a)}(u) \prod_{b \neq a} T^{(b)}(u)^{-C_{b a}} & \varepsilon(a) \neq \varepsilon\end{cases}
$$

By $(8.21),(8.23)$, and $\mathbb{T}_{0}\left(X_{r}\right)$ in $(8.4)$, we have $T^{(a)}(u+1)=\tau_{(-1)^{u}}\left(T^{(a)}(u)\right)$. The following fact is known ([B, Chapter V, 6.2], [FZ3, Lemma 2.1]):

$$
\underbrace{\cdots \tau_{-} \tau_{+} \tau_{-} \tau_{+}}_{h \text { times }}=\underbrace{\cdots \tau_{+} \tau_{-} \tau_{+} \tau_{-}}_{h \text { times }}=\omega_{0} \quad(\text { the longest element of } W)
$$

where $h$ is the Coxeter number of $X_{r}$. Also, $\omega_{0}\left(T^{(a)}(u)\right)=T^{(\omega(a))}(u)^{-1}$ due to the remark after (3.6). Using these results, we obtain

$$
\begin{aligned}
T^{(a)}(u+h) & =(\underbrace{\cdots \tau_{\mp} \tau_{ \pm} \tau_{\mp} \tau_{ \pm}}_{h \text { times }})\left(T^{(a)}(u)\right) \\
& =\omega_{0}\left(T^{(a)}(u)\right)=T^{(\omega(a))}(u)^{-1} .
\end{aligned}
$$

REMARK 8.9. As for the half-periodicity of $\mathcal{T}_{0}\left(X_{r}\right)$, a similar result has been obtained in [KNS2, (2.8)]. Compared with Theorem 8.8 here, the result there is weaker in that it does not cover $A_{r}(r \geq 2)$ or individual $T_{m}^{(a)}(u)$ for $E_{6}$ and $E_{8}$. Moreover, [KNS2, (2.8a)] should be corrected for $X_{r}=D_{r}$ with $r$ odd and $a=r-1, r$. 


\section{§9. Periodicities of restricted T- and Y-systems for twisted quan- tum affine algebras}

The T- and Y-systems considered so far are associated with the untwisted quantum affine algebra $U_{q}(\hat{\mathfrak{g}})$ (when $\left.U=U_{t \hbar}\right)$ as explained in Section 2 . In this section we consider $\mathrm{T}$ - and Y-systems associated with the twisted quantum affine algebra $U_{q}\left(\hat{\mathfrak{g}}^{\sigma}\right)$ following $[\mathrm{KS}]$, [Her2]. All the basic results presented for the untwisted case can be naturally extended to the twisted case as well. Moreover, the periodicity property of the twisted case reduces to that of the untwisted case.

\subsection{Dynkin diagrams of twisted affine type}

Throughout this section, we let $X_{N}$ exclusively denote a Dynkin diagram of type $A_{N}(N \geq 2), D_{N}(N \geq 4)$, or $E_{6}$. We keep the enumeration of the nodes of $X_{N}$ by the set $I=\{1, \ldots, N\}$ as in Figure 1 . For a pair $\left(X_{N}, \kappa\right)=$ $\left(A_{N}, 2\right),\left(D_{N}, 2\right),\left(E_{6}, 2\right)$, or $\left(D_{4}, 3\right)$, we define the diagram automorphism $\sigma: I \rightarrow I$ of $X_{N}$ of order $\kappa$ as $\sigma(a)=a$ except for the following cases (in our enumeration):

$$
\begin{array}{ll}
\sigma(a)=N+1-a \quad(a \in I) & \left(X_{N}, \kappa\right)=\left(A_{N}, 2\right), \\
\sigma(N-1)=N, \sigma(N)=N-1 & \left(X_{N}, \kappa\right)=\left(D_{N}, 2\right), \\
\sigma(1)=6, \sigma(2)=5, \sigma(5)=2, \sigma(6)=1 & \left(X_{N}, \kappa\right)=\left(E_{6}, 2\right), \\
\sigma(1)=3, \sigma(3)=4, \sigma(4)=1 & \left(X_{N}, \kappa\right)=\left(D_{4}, 3\right) .
\end{array}
$$

The map $\sigma$ is the same as the involution $\omega: I \rightarrow I$ in (3.6) except for $X_{N}=D_{N}\left(N\right.$ : even). Let $I / \sigma$ be the set of the $\sigma$-orbits of nodes of $X_{N}$. We choose, at our discretion, a complete set of representatives $I_{\sigma} \subset I$ of $I / \sigma$ as

$$
I_{\sigma}= \begin{cases}\{1,2, \ldots, r\} & \left(X_{N}, \kappa\right)=\left(A_{2 r-1}, 2\right),\left(A_{2 r}, 2\right),\left(D_{r+1}, 2\right) \\ \{1,2,3,4\} & \left(X_{N}, \kappa\right)=\left(E_{6}, 2\right) \\ \{1,2\} & \left(X_{N}, \kappa\right)=\left(D_{4}, 3\right)\end{cases}
$$

Let $X_{N}^{(\kappa)}=A_{2 r-1}^{(2)}(r \geq 2), A_{2 r}^{(2)}(r \geq 1), D_{r+1}^{(2)}(r \geq 3), E_{6}^{(2)}$, or $D_{4}^{(3)}$ be a Dynkin diagram of twisted affine type $[\mathrm{Ka}]$. We enumerate the nodes of $X_{N}^{(\kappa)}$ with $I_{\sigma} \cup\{0\}$ as in Figure 8, where $I_{\sigma}$ is the one for $\left(X_{N}, \kappa\right)$. By this, we have established the identification of the non-zeroth nodes of the diagram $X_{N}^{(\kappa)}$ with the nodes of the diagram $X_{N}$ belonging to the set $I_{\sigma}$. 

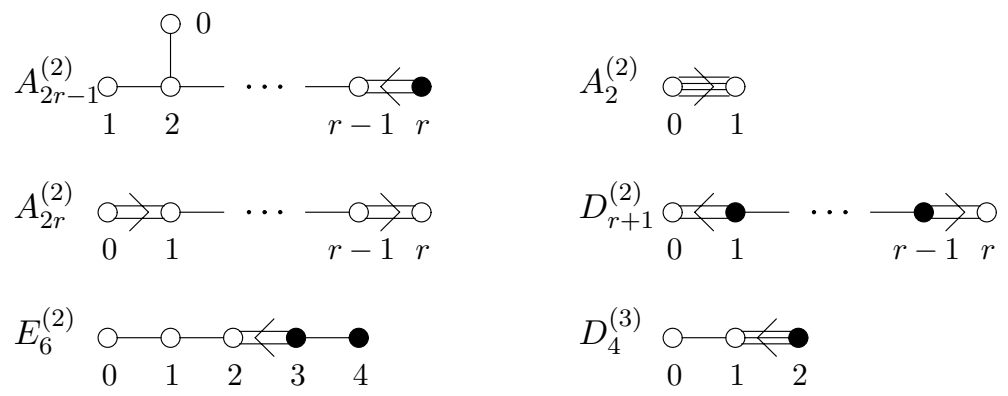

Figure 8: The Dynkin diagrams $X_{N}^{(\kappa)}$ of twisted affine type and their enumerations by $I_{\sigma} \cup\{0\}$. For a solid node $a, \sigma(a)=a$ (i.e., $\kappa_{a}=\kappa$ ) holds.

For example, for $E_{6}^{(2)}$, the correspondence is as follows:
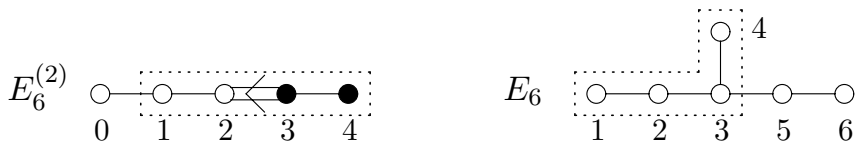

The solid nodes 3,4 in $E_{6}^{(2)}$ correspond to the fixed nodes by $\sigma$ in $E_{6}$. We use this identification throughout the section. (The zeroth node of $X_{N}^{(\kappa)}$ is irrelevant in our setting here.)

We define $\kappa_{a}\left(a \in I_{\sigma}\right)$ as

$$
\kappa_{a}= \begin{cases}1 & \sigma(a) \neq a, \\ \kappa & \sigma(a)=a .\end{cases}
$$

Note that $X_{N}^{(2)}=A_{2 r}^{(2)}$ is the unique case in which $\kappa_{a}=1$ for any $a \in I_{\sigma}$.

\subsection{Unrestricted T-systems}

Choose $\hbar \in \mathbb{C} \backslash 2 \pi \sqrt{-1} \mathbb{Q}$ arbitrarily.

Definition 9.1. The unrestricted T-system $\mathbb{T}\left(X_{N}^{(\kappa)}\right)$ of type $X_{N}^{(\kappa)}$ is the following system of relations for a family of variables $T=\left\{T_{m}^{(a)}(u) \mid a \in\right.$ $\left.I_{\sigma}, m \in \mathbb{N}, u \in \mathbb{C}_{\kappa_{a} \hbar}\right\}$, where $\Omega=2 \pi \sqrt{-1} / \kappa \hbar$, and $T_{m}^{(0)}(u)=T_{0}^{(a)}(u)=1$ if they occur in the right-hand sides in the relations.

For $X_{N}^{(\kappa)}=A_{2 r-1}^{(2)}$,

$$
\begin{array}{r}
T_{m}^{(a)}(u-1) T_{m}^{(a)}(u+1)=T_{m-1}^{(a)}(u) T_{m+1}^{(a)}(u)+T_{m}^{(a-1)}(u) T_{m}^{(a+1)}(u) \\
(1 \leq a \leq r-1)
\end{array}
$$




$$
\begin{aligned}
T_{m}^{(r)}(u-1) T_{m}^{(r)}(u+1)= & T_{m-1}^{(r)}(u) T_{m+1}^{(r)}(u) \\
& +T_{m}^{(r-1)}(u) T_{m}^{(r-1)}(u+\Omega) .
\end{aligned}
$$

For $X_{N}^{(\kappa)}=A_{2 r}^{(2)}$

$$
\begin{aligned}
T_{m}^{(a)}(u-1) T_{m}^{(a)}(u+1)= & T_{m-1}^{(a)}(u) T_{m+1}^{(a)}(u) \\
& +T_{m}^{(a-1)}(u) T_{m}^{(a+1)}(u) \quad(1 \leq a \leq r-1), \\
T_{m}^{(r)}(u-1) T_{m}^{(r)}(u+1)= & T_{m-1}^{(r)}(u) T_{m+1}^{(r)}(u)+T_{m}^{(r-1)}(u) T_{m}^{(r)}(u+\Omega) .
\end{aligned}
$$

For $X_{N}^{(\kappa)}=D_{r+1}^{(2)}$

$$
\begin{aligned}
T_{m}^{(a)}(u-1) T_{m}^{(a)}(u+1)= & T_{m-1}^{(a)}(u) T_{m+1}^{(a)}(u) \\
& +T_{m}^{(a-1)}(u) T_{m}^{(a+1)}(u) \quad(1 \leq a \leq r-2), \\
T_{m}^{(r-1)}(u-1) T_{m}^{(r-1)}(u+1)= & T_{m-1}^{(r-1)}(u) T_{m+1}^{(r-1)}(u) \\
& +T_{m}^{(r-2)}(u) T_{m}^{(r)}(u) T_{m}^{(r)}(u+\Omega) \\
T_{m}^{(r)}(u-1) T_{m}^{(r)}(u+1)= & T_{m-1}^{(r)}(u) T_{m+1}^{(r)}(u)+T_{m}^{(r-1)}(u) .
\end{aligned}
$$

For $X_{N}^{(\kappa)}=E_{6}^{(2)}$

$$
\begin{aligned}
T_{m}^{(1)}(u-1) T_{m}^{(1)}(u+1)= & T_{m-1}^{(1)}(u) T_{m+1}^{(1)}(u)+T_{m}^{(2)}(u), \\
T_{m}^{(2)}(u-1) T_{m}^{(2)}(u+1)= & T_{m-1}^{(2)}(u) T_{m+1}^{(2)}(u)+T_{m}^{(1)}(u) T_{m}^{(3)}(u), \\
T_{m}^{(3)}(u-1) T_{m}^{(3)}(u+1)= & T_{m-1}^{(3)}(u) T_{m+1}^{(3)}(u) \\
& +T_{m}^{(2)}(u) T_{m}^{(2)}(u+\Omega) T_{m}^{(4)}(u), \\
T_{m}^{(4)}(u-1) T_{m}^{(4)}(u+1)= & T_{m-1}^{(4)}(u) T_{m+1}^{(4)}(u)+T_{m}^{(3)}(u) .
\end{aligned}
$$

For $X_{N}^{(\kappa)}=D_{4}^{(3)}$

$$
\begin{aligned}
T_{m}^{(1)}(u-1) T_{m}^{(1)}(u+1)= & T_{m-1}^{(1)}(u) T_{m+1}^{(1)}(u)+T_{m}^{(2)}(u), \\
T_{m}^{(2)}(u-1) T_{m}^{(2)}(u+1)= & T_{m-1}^{(2)}(u) T_{m+1}^{(2)}(u) \\
& +T_{m}^{(1)}(u) T_{m}^{(1)}(u-\Omega) T_{m}^{(1)}(u+\Omega) .
\end{aligned}
$$


The domain $\mathbb{C}_{\kappa_{a} \hbar}$ of the parameter $u$ effectively imposes the following periodic condition:

$$
T_{m}^{(a)}(u)= \begin{cases}T_{m}^{(a)}(u+\kappa \Omega) & \sigma(a) \neq a \\ T_{m}^{(a)}(u+\Omega) & \sigma(a)=a\end{cases}
$$

Definition 9.2. The unrestricted T-algebra $\mathcal{T}\left(X_{N}^{(\kappa)}\right)$ of type $X_{N}^{(\kappa)}$ is the ring with generators $T_{m}^{(a)}(u)^{ \pm 1}\left(a \in I_{\sigma}, m \in \mathbb{N}, u \in \mathbb{C}_{\kappa_{a} \hbar}\right)$ and the relations $\mathbb{T}\left(X_{N}^{(\kappa)}\right)$. Also, we define the ring $\mathcal{T}^{\circ}\left(X_{N}^{(\kappa)}\right)$ as the subring of $\mathcal{T}\left(X_{N}^{(\kappa)}\right)$ generated by $T_{m}^{(a)}(u)\left(a \in I_{\sigma}, m \in \mathbb{N}, u \in \mathbb{C}_{\kappa_{a} \hbar}\right)$.

The following are some features of the T-system $\mathbb{T}\left(X_{N}^{(\kappa)}\right)$ that are specific to the twisted case.

(i) The relations include the two basic units of the parameter $u, 1$ and $\Omega$, which are $\mathbb{Z}$-linearly independent under our assumption of $\hbar \notin 2 \pi \sqrt{-1} \mathbb{Q}$.

(ii) The domain $\mathbb{C}_{\kappa_{a}} \hbar$ of the parameter $u$ and the resulting periodic condition (9.10) depend on $a \in I_{\sigma}$.

(iii) We do not consider the T-system $\mathbb{T}\left(X_{N}^{(\kappa)}\right)$ whose domain $U$ of the parameter $u$ is $\mathbb{C}$. This is because the periodic condition (9.10) is now an integral part of the relations $\mathbb{T}\left(X_{N}^{(\kappa)}\right)$ due to (i). This is also natural given that no Yangian analogue of the twisted quantum affine algebra $U_{q}\left(\hat{\mathfrak{g}}^{\sigma}\right)$ is known.

(iv) The discrete version of $\mathbb{T}\left(X_{N}^{(\kappa)}\right)$ is available by taking the domain $U$ of the parameter $u$ as $U=\mathbb{Z} \times \mathbb{Z}_{\kappa}$, where $(a, b) \in U$ corresponds to $u=$ $a+b \Omega$, and imposing the periodic condition (9.10).

REMARK 9.3. The T-system $\mathbb{T}\left(X_{N}^{(\kappa)}\right)$ was introduced in $[\mathrm{KS}]$ as a family of relations in the ring of the commuting transfer matrices for solvable lattice models associated with the twisted quantum affine algebra $U_{q}\left(\hat{\mathfrak{g}}^{\sigma}\right)$ of type $X_{N}^{(\kappa)}$.

REMARK 9.4. Unifying the untwisted and twisted cases, the T-system $\mathbb{T}\left(X_{r}\right)$ and the Y-system $\mathbb{Y}\left(X_{r}\right)$ of type $X_{r}$ in Section 2 are also said to be of type $X_{r}^{(1)}$ and are denoted by $\mathbb{T}\left(X_{r}^{(1)}\right)$ and $\mathbb{Y}\left(X_{r}^{(1)}\right)$. Strictly speaking, this should be applied only when the domain $U$ of the parameter $u$ is $\mathbb{C}_{t \hbar}$. However, as we have seen, such a distinction of $U$ is not so essential in many aspects of $\mathbb{T}\left(X_{r}\right)$ and $\mathbb{Y}\left(X_{r}\right)$. 
There is a simple relation between the rings $\mathcal{T}\left(X_{N}^{(\kappa)}\right)$ and $\mathcal{T}\left(X_{N}\right)$. Let $\left\{\hat{T}_{m}^{(a)}(u)^{ \pm 1} \mid a \in I, m \in \mathbb{N}, u \in \mathbb{C}_{\hbar}\right\}$ be the set of generators of $\mathcal{T}\left(X_{N}\right)$. Let $\mathcal{J}^{\sigma}$ be the ideal of $\mathcal{T}\left(X_{N}\right)$ generated by

$$
\hat{T}_{m}^{(a)}(u)-\hat{T}_{m}^{(\sigma(a))}(u+\Omega) \quad\left(a \in I, m \in \mathbb{N}_{\geq 1}, u \in \mathbb{C}_{\hbar}\right) .
$$

Then one can choose a generating set of $\mathcal{T}\left(X_{N}\right) / \mathcal{J}^{\sigma}$ as $\left\{\hat{T}_{m}^{(a)}(u)^{ \pm 1} \mid a \in\right.$ $\left.I_{\sigma}, m \in \mathbb{Z}_{\geq 1}, u \in \mathbb{C}_{\kappa_{a} \hbar}\right\}$.

Proposition 9.5. There is a ring isomorphism

$$
\begin{aligned}
\mathcal{T}\left(X_{N}\right) / \mathcal{J}^{\sigma} & \rightarrow \mathcal{T}\left(X_{N}^{(\kappa)}\right), \\
\hat{T}_{m}^{(a)}(u) & \mapsto T_{m}^{(a)}(u) \quad\left(a \in I_{\sigma}\right) .
\end{aligned}
$$

Proof. It is easy to check that the relations of the both rings are identical under the correspondence.

The T-system $\mathbb{T}\left(X_{N}^{(\kappa)}\right)$ plays the same role in the Grothendieck ring $\operatorname{Rep} U_{q}\left(\hat{\mathfrak{g}}^{\sigma}\right)$ of the category of type 1 finite-dimensional $U_{q}\left(\hat{\mathfrak{g}}^{\sigma}\right)$-modules for the twisted quantum affine algebra $U_{q}\left(\hat{\mathfrak{g}}^{\sigma}\right)$ of type $X_{N}^{(\kappa)}$ as does the untwisted case.

For arbitrarily chosen $\hbar \in \mathbb{C} \backslash 2 \pi \sqrt{-1} \mathbb{Q}$, we set the deformation parameter $q$ of the twisted quantum affine algebras $U_{q}(\hat{\mathfrak{g}})[\mathrm{J}],[\mathrm{D} 1],[\mathrm{D} 2]$ as $q=e^{\hbar} \in \mathbb{C}^{\times}$, so that $q$ is not a root of unity.

The $q$-character map $\chi_{q}^{\sigma}$ of $U_{q}\left(\hat{\mathfrak{g}}^{\sigma}\right)$ is defined by Hernandez [Her2] as an injective ring homomorphism

$$
\chi_{q}^{\sigma}: \operatorname{Rep} U_{q}\left(\hat{\mathfrak{g}}^{\sigma}\right) \rightarrow \mathbb{Z}\left[Z_{i, a}^{ \pm 1}\right]_{i \in I_{\sigma}, a \in \mathbb{C}^{\times}}
$$

Consult [Her2] for more information on $U_{q}\left(\hat{\mathfrak{g}}^{\sigma}\right)$ and $\chi_{q}^{\sigma}$. The enumeration of $I_{\sigma}$ in [Her2] is the same as the present one except for $A_{2 r}^{(2)}$, where $1,2, \ldots, r$ here correspond to $r-1, \ldots, 1,0$ in [Her2]. To make the description uniform, for $a \in I_{\sigma}=\{1, \ldots, r\}$ we set $\bar{a}=r-a$ for $X_{N}^{(\kappa)}=A_{2 r}^{(2)}$ and $\bar{a}=a$ otherwise. (This notation $\bar{a}$ will be used only in the rest of this section.) From now on, we employ the parameterization of the variables $Z_{\bar{a}, q^{\kappa_{a} u}}\left(a \in I_{\sigma}, u \in \mathbb{C}_{\kappa_{a} \hbar}\right)$ instead of $Z_{i, a}\left(i \in I_{\sigma}, a \in \mathbb{C}^{\times}\right)$in [Her2]. The $q$-character ring $\mathrm{Ch} U_{q}\left(\hat{\mathfrak{g}}^{\sigma}\right)$ of $U_{q}\left(\hat{\mathfrak{g}}^{\sigma}\right)$ is defined to be $\operatorname{Im} \chi_{q}^{\sigma}$. 
Definition 9.6. A Kirillov-Reshetikhin module $W_{m}^{(a)}(u)\left(a \in I_{\sigma}, m \in\right.$ $\left.\mathbb{N}, u \in \mathbb{C}_{\kappa_{a} \hbar}\right)$ of $U_{q}\left(\hat{\mathfrak{g}}^{\sigma}\right)$ of type $X_{N}^{(\kappa)}$ is the irreducible finite dimensional $U_{q}\left(\hat{\mathfrak{g}}^{\sigma}\right)$-module with highest weight monomial

$$
\prod_{j=1}^{m} Z_{\bar{a}, q^{\kappa_{a}}(u+m+1-2 j)} .
$$

REMARK 9.7. The above $W_{m}^{(a)}(u)$ corresponds to $W_{m, q^{\kappa a}(u-m+1)}^{(\bar{a})}$ in [Her2]. The $T$-system $\mathbb{T}\left(X_{N}^{(\kappa)}\right)$ in Definition 9.1 agrees with the one in [Her2, Section 4.3] under the identification $T_{m}^{(a)}(u)=X_{m, q^{\kappa a}(u-m+1)}^{(\bar{a})}$.

In the same way as (2.9), we define $S_{a m u}(T) \in \mathbb{Z}[T] \quad(a \in I, m \in \mathbb{N}, u \in$ $\left.\mathbb{C}_{\kappa_{a} \hbar}\right)$, so that all the relations in $\mathbb{T}\left(X_{N}^{(\kappa)}\right)$ are written in the form $S_{a m u}(T)=$ 0 . Let $I\left(\mathbb{T}\left(X_{N}^{(\kappa)}\right)\right)$ be the ideal of $\mathbb{Z}[T]$ generated by $S_{a m u}(T)$.

TheOrem 9.8. Let $\widetilde{T}=\left\{\widetilde{T}_{m}^{(a)}(u):=\chi_{q}^{\sigma}\left(W_{m}^{(a)}(u)\right) \mid a \in I_{\sigma}, m \in \mathbb{N}, u \in \mathbb{C}_{\kappa_{a} \hbar}\right\}$ be the family of the q-characters of the Kirillov-Reshetikhin modules of $U_{q}\left(\hat{\mathfrak{g}}^{\sigma}\right)$ of type $X_{N}^{(\kappa)}$. Then,

(1) the family $\widetilde{T}$ generates the ring $\mathrm{Ch} U_{q}\left(\hat{\mathfrak{g}}^{\sigma}\right)$;

(2) (Hernandez [Her2]) the family $\widetilde{T}$ satisfies the T-system $\mathbb{T}\left(X_{N}^{(\kappa)}\right)$ in Ch $U_{q}\left(\hat{\mathfrak{g}}^{\sigma}\right)$ (by replacing $T_{m}^{(a)}(u)$ in $\mathbb{T}\left(X_{N}^{(\kappa)}\right)$ with $\widetilde{T}_{m}^{(a)}(u)$ );

(3) for any $P(T) \in \mathbb{Z}[T]$, the relation $P(\widetilde{T})=0$ holds in $\operatorname{Ch} U_{q}\left(\hat{\mathfrak{g}}^{\sigma}\right)$ if and only if there is a nonzero monomial $M(T) \in \mathbb{Z}[T]$ such that $M(T) P(T) \in$ $I\left(\mathbb{T}\left(X_{N}^{(\kappa)}\right)\right)$.

Proof. (1) The fundamental character $\chi_{q}^{\sigma}\left(W_{1}^{(a)}(u)\right)$ has the form

$$
\chi_{q}^{\sigma}\left(W_{1}^{(a)}(u)\right)=Z_{\bar{a}, q^{\kappa a u}}+(\text { lower term }),
$$

where "lower" means lower in the weight lattice for the subalgebra $U_{q}\left(\mathfrak{g}^{\sigma}\right)$ of $U_{q}\left(\hat{\mathfrak{g}}^{\sigma}\right)$ [Her2]. Thus, if there is a nontrivial relation among the fundamental characters, then it causes some nontrivial relation among $Z_{\bar{a}, q^{\kappa_{a} u}}$ 's. This is a contradiction.

(2) This was proved by [Her2, Theorem 4.2].

(3) The proof is completely parallel with Theorem $2.8(3)$ by setting the height as ht $T_{m}^{(a)}(u)=m$.

Corollary 9.9. The ring $\mathcal{T}^{\circ}\left(X_{N}^{(\kappa)}\right)$ is isomorphic to $\operatorname{Rep} U_{q}\left(\hat{\mathfrak{g}}^{\sigma}\right)$ by the correspondence $T_{m}^{(a)}(u) \mapsto W_{m}^{(a)}(u)$. 
In Appendix A.2 we give parallel results for the ring associated with the Q-system and $\operatorname{Rep} U_{q}\left(\mathfrak{g}^{\sigma}\right)$.

\subsection{Unrestricted Y-systems}

Definition 9.10. The unrestricted $Y$-system $\mathbb{T}\left(X_{N}^{(\kappa)}\right)$ of type $X_{N}^{(\kappa)}$ is the following system of relations for a family of variables $Y=\left\{Y_{m}^{(a)}(u) \mid a \in\right.$ $\left.I_{\sigma}, m \in \mathbb{N}, u \in \mathbb{C}_{\kappa_{a} \hbar}\right\}$, where $\Omega=2 \pi \sqrt{-1} / \kappa \hbar$, and $Y_{m}^{(0)}(u)=Y_{0}^{(a)}(u)^{-1}=0$ if they occur in the right-hand sides in the following relations.

For $X_{N}^{(\kappa)}=A_{2 r-1}^{(2)}$,

$$
\begin{aligned}
Y_{m}^{(a)}(u-1) Y_{m}^{(a)}(u+1) & =\frac{\left(1+Y_{m}^{(a-1)}(u)\right)\left(1+Y_{m}^{(a+1)}(u)\right)}{\left(1+Y_{m-1}^{(a)}(u)^{-1}\right)\left(1+Y_{m+1}^{(a)}(u)^{-1}\right)} \\
(1 \leq a \leq r-1), & \left(1 \leq Y_{m}^{(r-1)}(u)\right)\left(1+Y_{m}^{(r-1)}(u+\Omega)\right) \\
Y_{m}^{(r)}(u-1) Y_{m}^{(r)}(u+1)= & \left(1+Y_{m-1}^{(r)}(u)^{-1}\right)\left(1+Y_{m+1}^{(r)}(u)^{-1}\right)
\end{aligned}
$$

For $X_{N}^{(\kappa)}=A_{2 r}^{(2)}$

$$
\begin{aligned}
& Y_{m}^{(a)}(u-1) Y_{m}^{(a)}(u+1)=\frac{\left(1+Y_{m}^{(a-1)}(u)\right)\left(1+Y_{m}^{(a+1)}(u)\right)}{\left(1+Y_{m-1}^{(a)}(u)^{-1}\right)\left(1+Y_{m+1}^{(a)}(u)^{-1}\right)} \\
& \quad(1 \leq a \leq r-1) \\
& Y_{m}^{(r)}(u-1) Y_{m}^{(r)}(u+1)=\frac{\left(1+Y_{m}^{(r-1)}(u)\right)\left(1+Y_{m}^{(r)}(u+\Omega)\right)}{\left(1+Y_{m-1}^{(r)}(u)^{-1}\right)\left(1+Y_{m+1}^{(r)}(u)^{-1}\right)}
\end{aligned}
$$

For $X_{N}^{(\kappa)}=D_{r+1}^{(2)}$,

$$
\begin{aligned}
Y_{m}^{(a)}(u-1) Y_{m}^{(a)}(u+1)= & \frac{\left(1+Y_{m}^{(a-1)}(u)\right)\left(1+Y_{m}^{(a+1)}(u)\right)}{\left(1+Y_{m-1}^{(a)}(u)^{-1}\right)\left(1+Y_{m+1}^{(a)}(u)^{-1}\right)} \\
(1 \leq a \leq r-2), & \left(1+Y_{m}^{(r-2)}(u)\right)\left(1+Y_{m}^{(r)}(u)\right) \\
Y_{m}^{(r-1)}(u-1) Y_{m}^{(r-1)}(u+1)= & \frac{\times\left(1+Y_{m}^{(r)}(u+\Omega)\right)}{\left(1+Y_{m-1}^{(r-1)}(u)^{-1}\right)\left(1+Y_{m+1}^{(r-1)}(u)^{-1}\right)}, \\
Y_{m}^{(r)}(u-1) Y_{m}^{(r)}(u+1)= & \frac{1+Y_{m}^{(r-1)}(u)}{\left(1+Y_{m-1}^{(r)}(u)^{-1}\right)\left(1+Y_{m+1}^{(r)}(u)^{-1}\right)}
\end{aligned}
$$


For $X_{N}^{(\kappa)}=E_{6}^{(2)}$

$$
\begin{aligned}
Y_{m}^{(1)}(u-1) Y_{m}^{(1)}(u+1)= & \frac{1+Y_{m}^{(2)}(u)}{\left(1+Y_{m-1}^{(1)}(u)^{-1}\right)\left(1+Y_{m+1}^{(1)}(u)^{-1}\right)}, \\
Y_{m}^{(2)}(u-1) Y_{m}^{(2)}(u+1)= & \frac{\left(1+Y_{m}^{(1)}(u)\right)\left(1+Y_{m}^{(3)}(u)\right)}{\left(1+Y_{m-1}^{(2)}(u)^{-1}\right)\left(1+Y_{m+1}^{(2)}(u)^{-1}\right)}, \\
Y_{m}^{(3)}(u-1) Y_{m}^{(3)}(u+1)= & \frac{\left(1+Y_{m}^{(2)}(u)\right)\left(1+Y_{m}^{(2)}(u+\Omega)\right)}{\left(1+Y_{m-1}^{(3)}(u)^{-1}\right)\left(1+Y_{m+1}^{(3)}(u)^{-1}\right)}, \\
Y_{m}^{(4)}(u-1) Y_{m}^{(4)}(u+1)= & \frac{1+Y_{m}^{(3)}(u)}{\left(1+Y_{m-1}^{(4)}(u)^{-1}\right)\left(1+Y_{m+1}^{(4)}(u)^{-1}\right)} .
\end{aligned}
$$

For $X_{N}^{(\kappa)}=D_{4}^{(3)}$

$$
\begin{aligned}
Y_{m}^{(1)}(u-1) Y_{m}^{(1)}(u+1)= & \frac{1+Y_{m}^{(2)}(u)}{\left(1+Y_{m-1}^{(1)}(u)^{-1}\right)\left(1+Y_{m+1}^{(1)}(u)^{-1}\right)}, \\
& \left(1+Y_{m}^{(1)}(u)\right)\left(1+Y_{m}^{(1)}(u-\Omega)\right) \\
Y_{m}^{(2)}(u-1) Y_{m}^{(2)}(u+1)= & \frac{\times\left(1+Y_{m}^{(1)}(u+\Omega)\right)}{\left(1+Y_{m-1}^{(2)}(u)^{-1}\right)\left(1+Y_{m+1}^{(2)}(u)^{-1}\right)} .
\end{aligned}
$$

The domain $\mathbb{C}_{\kappa_{a} \hbar}$ of the parameter $u$ effectively imposes the following periodic condition:

$$
Y_{m}^{(a)}(u)= \begin{cases}Y_{m}^{(a)}(u+\kappa \Omega) & \sigma(a) \neq a \\ Y_{m}^{(a)}(u+\Omega) & \sigma(a)=a .\end{cases}
$$

Definition 9.11. The unrestricted $Y$-algebra $y\left(X_{N}^{(\kappa)}\right)$ of type $X_{N}^{(\kappa)}$ is the ring with generators $Y_{m}^{(a)}(u)^{ \pm 1},\left(1+Y_{m}^{(a)}(u)\right)^{-1}\left(a \in I_{\sigma}, m \in \mathbb{N}, u \in \mathbb{C}_{\kappa_{a} \hbar}\right)$ and the relations $\mathbb{Y}\left(X_{N}^{(\kappa)}\right)$.

Let $\left\{\hat{Y}_{m}^{(a)}(u)^{ \pm 1},\left(1+\hat{Y}_{m}^{(a)}(u)\right)^{-1} \mid a \in I, m \in \mathbb{N}, u \in \mathbb{C}_{\hbar}\right\}$ be the set of generators of $y\left(X_{N}\right)$. Let $\mathcal{J}^{\sigma}$ be the ideal of $y\left(X_{N}\right)$ generated by

$$
\hat{Y}_{m}^{(a)}(u)-\hat{Y}_{m}^{(\sigma(a))}(u+\Omega) \quad\left(a \in I, m \in \mathbb{N}, u \in \mathbb{C}_{\hbar}\right) .
$$


Then one can choose a generating set of $y\left(X_{N}\right) / \mathcal{J}^{\sigma}$ as $\left\{\hat{Y}_{m}^{(a)}(u)^{ \pm 1},(1+\right.$ $\left.\left.\hat{Y}_{m}^{(a)}(u)\right)^{-1} \mid a \in I_{\sigma}, m \in \mathbb{N}, u \in \mathbb{C}_{\kappa_{a} \hbar}\right\}$.

As Proposition 9.5, we have the following.

Proposition 9.12. There is a ring isomorphism

$$
\begin{aligned}
y\left(X_{N}\right) / \mathcal{J}^{\sigma} & \rightarrow y\left(X_{N}^{(\kappa)}\right), \\
\hat{Y}_{m}^{(a)}(u) & \mapsto Y_{m}^{(a)}(u) \quad\left(a \in I_{\sigma}\right) .
\end{aligned}
$$

The following theorem is an analogue of Theorem 2.12.

THEOREM 9.13. (1) There is a ring homomorphism

$$
\varphi: y\left(X_{N}^{(\kappa)}\right) \rightarrow \mathcal{T}\left(X_{N}^{(\kappa)}\right)
$$

defined by

$$
Y_{m}^{(a)}(u) \mapsto \frac{M_{m}^{(a)}(u)}{T_{m-1}^{(a)}(u) T_{m+1}^{(a)}(u)},
$$

where $T_{0}^{(a)}(u)=1$.

(2) There is a ring homomorphism

$$
\psi: \mathcal{T}\left(X_{N}^{(\kappa)}\right) \rightarrow y\left(X_{N}^{(\kappa)}\right)
$$

such that $\psi \circ \varphi=\operatorname{id}_{y\left(X_{N}^{(\kappa)}\right)}$.

Proof. We derive the theorem from the results of Theorem 2.12 and Propositions 9.5 and 9.12 .

(1) Let $\hat{\varphi}: y\left(X_{N}\right) \rightarrow \mathcal{T}\left(X_{N}\right)$ be the homomorphism in (2.21). Let $g^{\sigma}$ and $\mathcal{J}^{\sigma}$ be the ideals of $\mathcal{T}\left(X_{N}\right)$ and $\mathcal{y}\left(X_{N}\right)$ in Propositions 9.5 and 9.12 , respectively. We claim that $\hat{\varphi}\left(\mathcal{J}^{\sigma}\right) \subset \mathcal{J}^{\sigma}$. In fact,

$$
\begin{aligned}
\hat{\varphi}\left(\hat{Y}_{m}^{(a)}(u)\right) & =\frac{\hat{M}_{m}^{(a)}(u)}{\hat{T}_{m-1}^{(a)}(u) \hat{T}_{m+1}^{(a)}(u)} \\
& \equiv \frac{\hat{M}_{m}^{(\sigma(a))}(u+\Omega)}{\hat{T}_{m-1}^{(\sigma(a))}(u+\Omega) \hat{T}_{m+1}^{(\sigma(a))}(u+\Omega)} \quad \bmod \mathcal{J}^{\sigma} \\
& =\hat{\varphi}\left(\hat{Y}_{m}^{(\sigma(a))}(u+\Omega)\right),
\end{aligned}
$$


where we also used the invariance of $\mathbb{T}\left(X_{N}\right)$ by $\sigma$ in the second equality. Then, the induced homomorphism

$$
\hat{\varphi}^{\sigma}: y\left(X_{N}\right) / \mathcal{J}^{\sigma} \rightarrow \mathcal{T}\left(X_{N}\right) / \mathcal{J}^{\sigma}
$$

gives the desired homomorphism $\varphi$ under the isomorphisms in Propositions 9.5 and 9.12 .

(2) Let $\hat{\psi}: \mathcal{T}\left(X_{N}\right) \rightarrow y\left(X_{N}\right)$ be the homomorphism in (2.25), where we modify Step 1 of the construction of $\hat{\psi}$ in the proof of Theorem 2.12 with the following. (For simplicity, we write $\hat{\psi}\left(T_{1}^{(a)}(u)\right)$ as $T_{1}^{(a)}(u)$.)

Step 1. We arbitrarily choose $T_{1}^{(a)}(u) \in \mathcal{y}\left(X_{N}\right)^{\times}(a \in I)$ for each $u \in \mathbb{C}_{\hbar}$ in the region $-1 \leq \operatorname{Re} u<1$ such that

$$
T_{1}^{(a)}(u) \equiv T_{1}^{(\sigma(a))}(u+\Omega) \bmod \mathcal{J}^{\sigma} .
$$

(For example, just take $T_{1}^{(a)}(u)=1$.)

Then, one can easily show that $T_{m}^{(a)}(u) \equiv T_{m}^{(\sigma(a))}(u+\Omega) \bmod \mathcal{J}^{\sigma}$ for any $T_{m}^{(a)}(u)$ constructed in Steps 2 and 3 of Theorem 2.12, again by the invariance of $\mathbb{T}\left(X_{N}\right)$ by $\sigma$. Then, the induced isomorphism

$$
\hat{\psi}^{\sigma}: \mathcal{T}\left(X_{N}\right) / \mathcal{J}^{\sigma} \rightarrow y\left(X_{N}\right) / \mathcal{J}^{\sigma}
$$

gives the desired homomorphism $\psi$ under the isomorphisms in Propositions 9.5 and 9.12. The property $\psi \circ \varphi=\operatorname{id}_{y\left(X_{N}^{(\kappa)}\right)}$ follows from $\hat{\psi}^{\sigma} \circ \hat{\varphi}^{\sigma}=$ $\operatorname{idy}\left(X_{N}\right) / J^{\sigma}$.

\subsection{Restricted T- and Y-systems}

Definition 9.14. Fix an integer $\ell \geq 2$. The level $\ell$ restricted T-system $\mathbb{T}_{\ell}\left(X_{N}^{(\kappa)}\right)$ of type $X_{N}^{(\kappa)}$ with the unit boundary condition is the system of relations (9.5)-(9.9) naturally restricted to a family of variables $T=\left\{T_{m}^{(a)}(u) \mid\right.$ $\left.a \in I_{\sigma} ; m=1, \ldots, \ell-1 ; u \in \mathbb{C}_{\kappa_{a} \hbar}\right\}$, where $T_{m}^{(0)}(u)=T_{0}^{(a)}(u)=1$, and furthermore, $T_{\ell}^{(a)}(u)=1$ (the unit boundary condition) if they occur in the right-hand sides in the relations.

Definition 9.15. The level $\ell$ restricted T-algebra $\mathcal{T}_{\ell}\left(X_{N}^{(\kappa)}\right)$ of type $X_{N}^{(\kappa)}$ is the ring with generators $T_{m}^{(a)}(u)^{ \pm 1}\left(a \in I_{\sigma} ; m=1, \ldots, \ell-1 ; u \in \mathbb{C}_{\kappa_{a} \hbar}\right)$ and the relations $\mathbb{T}_{\ell}\left(X_{N}^{(\kappa)}\right)$. Also, we define the ring $\mathcal{T}_{\ell}^{\circ}\left(X_{N}^{(\kappa)}\right)$ as the subring of $\mathcal{T}_{\ell}\left(X_{N}^{(\kappa)}\right)$ generated by $T_{m}^{(a)}(u)\left(a \in I_{\sigma} ; m=1, \ldots, \ell-1 ; u \in \mathbb{C}_{\kappa_{a} \hbar}\right)$. 
Definition 9.16. Fix an integer $\ell \geq 2$. The level $\ell$ restricted $Y$-system $\mathbb{Y}_{\ell}\left(X_{N}^{(\kappa)}\right)$ of type $X_{N}^{(\kappa)}$ is the system of relations (9.16)-(9.20) naturally restricted to a family of variables $Y=\left\{Y_{m}^{(a)}(u) \mid a \in I_{\sigma} ; m=1, \ldots, \ell-1 ; u \in\right.$ $\left.\mathbb{C}_{\kappa_{a} \hbar}\right\}$, where $Y_{m}^{(0)}(u)=Y_{0}^{(a)}(u)^{-1}=0$, and furthermore, $Y_{\ell}^{(a)}(u)^{-1}=0$ if they occur in the right-hand sides in the relations.

Definition 9.17. The level $\ell$ restricted $Y$-algebra $y_{\ell}\left(X_{N}^{(\kappa)}\right)$ of type $X_{N}^{(\kappa)}$ is the ring with generators $Y_{m}^{(a)}(u)^{ \pm 1},\left(1+Y_{m}^{(a)}(u)\right)^{-1}\left(a \in I_{\sigma} ; m=1, \ldots, \ell-\right.$ $\left.1 ; u \in \mathbb{C}_{\kappa_{a} \hbar}\right)$ and the relations $\mathbb{Y}_{\ell}\left(X_{N}^{(\kappa)}\right)$.

REMARK 9.18. The level restrictions of the T- and Y-systems for the twisted case are introduced here for the first time. The former is defined such that dropping the parameter $u$, namely, the formal replacement $T_{m}^{(a)}(u) \rightarrow$ $Q_{m}^{(a)}$, coincides with the level $\ell$ restricted Q-system introduced in [HKOTT, $(6.2)]$.

Propositions 9.5 and 9.12 have natural counterparts in the level restricted situation. Let $\left\{\hat{T}_{m}^{(a)}(u)^{ \pm 1} \mid a \in I, m=1, \ldots, \ell-1, u \in \mathbb{C}_{\hbar}\right\}$ be the set of generators of $\mathcal{T}_{\ell}\left(X_{N}\right)$. Let $\mathcal{J}_{\ell}^{\sigma}$ be the ideal of $\mathcal{T}_{\ell}\left(X_{N}\right)$ generated by

$$
\hat{T}_{m}^{(a)}(u)-\hat{T}_{m}^{(\sigma(a))}(u+\Omega) \quad\left(a \in I ; m=1, \ldots, l-1 ; u \in \mathbb{C}_{\hbar}\right) .
$$

Then one can choose a generating set of $\mathcal{T}_{\ell}\left(X_{N}\right) / \mathcal{J}_{\ell}^{\sigma}$ as $\left\{\hat{T}_{m}^{(a)}(u)^{ \pm 1} \mid a \in\right.$ $\left.I_{\sigma} ; m=1, \ldots, \ell-1 ; u \in \mathbb{C}_{\kappa_{a} \hbar}\right\}$.

Similarly, let $\left\{\hat{Y}_{m}^{(a)}(u)^{ \pm 1},\left(1+\hat{Y}_{m}^{(a)}(u)\right)^{-1} \mid a \in I ; m=1, \ldots, \ell-1 ; u \in \mathbb{C}_{\hbar}\right\}$ be the set of generators of $y_{\ell}\left(X_{N}\right)$. Let $\mathcal{J}_{\ell}^{\sigma}$ be the ideal of $y_{\ell}\left(X_{N}\right)$ generated by

$$
\hat{Y}_{m}^{(a)}(u)-\hat{Y}_{m}^{(\sigma(a))}(u+\Omega) \quad\left(a \in I ; m=1, \ldots, \ell-1 ; u \in \mathbb{C}_{\hbar}\right) .
$$

Then one can choose a generating set of $y_{\ell}\left(X_{N}\right) / \mathcal{J}_{\ell}^{\sigma}$ as $\left\{\hat{Y}_{m}^{(a)}(u)^{ \pm 1},(1+\right.$ $\left.\left.\hat{Y}_{m}^{(a)}(u)\right)^{-1} \mid a \in I_{\sigma} ; m=1, \ldots, \ell-1 ; u \in \mathbb{C}_{\kappa_{a} \hbar}\right\}$.

Proposition 9.19. There is a ring isomorphism

$$
\begin{aligned}
\mathcal{T}_{\ell}\left(X_{N}\right) / \mathcal{J}_{\ell}^{\sigma} & \rightarrow \mathcal{T}_{\ell}\left(X_{N}^{(\kappa)}\right), \\
\hat{T}_{m}^{(a)}(u) & \mapsto T_{m}^{(a)}(u) \quad\left(a \in I_{\sigma}\right) .
\end{aligned}
$$

Similarly, there is a ring isomorphism

$$
\begin{aligned}
y_{\ell}\left(X_{N}\right) / \mathcal{J}_{\ell}^{\sigma} & \rightarrow y_{\ell}\left(X_{N}^{(\kappa)}\right), \\
\hat{Y}_{m}^{(a)}(u) & \mapsto Y_{m}^{(a)}(u) \quad\left(a \in I_{\sigma}\right) .
\end{aligned}
$$


Proof. It is compatible to set $\hat{T}_{\ell}^{(a)}(u)=T_{\ell}^{(a)}(u)=1$ in Proposition 9.5. It is also compatible to set $\hat{Y}_{\ell}^{(a)}(u)^{-1}=Y_{\ell}^{(a)}(u)^{-1}=0$ in Proposition 9.12.

\subsection{Periodicities of restricted $\mathbf{T}$ - and $\mathrm{Y}$-systems}

Let $h^{\vee}$ be the dual Coxeter number of $X_{N}^{(\kappa)}[\mathrm{Ka}]$. It is the same with the (dual) Coxeter number of $X_{N}$ and the following:

\begin{tabular}{c|ccccc}
$X_{N}^{(\kappa)}$ & $A_{2 r-1}^{(2)}$ & $A_{2 r}^{(2)}$ & $D_{r+1}^{(2)}$ & $E_{6}^{(2)}$ & $D_{4}^{(3)}$ \\
\hline$h^{\vee}$ & $2 r$ & $2 r+1$ & $2 r$ & 12 & 6
\end{tabular}

The periodicity of $\mathcal{T}_{\ell}\left(X_{N}^{(\kappa)}\right)$ reduces to that of $\mathcal{T}_{\ell}\left(X_{N}\right)$ proved in Corollary 4.29 .

THEOREM 9.20. The following relations hold in $\mathcal{T}_{\ell}\left(X_{N}^{(\kappa)}\right)$ :

(1) Half-periodicity:

$$
T_{m}^{(a)}\left(u+h^{\vee}+\ell\right)= \begin{cases}T_{\ell-m}^{(a)}(u) & \text { if } X_{N}^{(\kappa)}=D_{r+1}^{(2)}(r+1: \text { even }) \text { or } D_{4}^{(3)} \\ T_{\ell-m}^{(a)}(u+\Omega) & \text { otherwise. }\end{cases}
$$

(2) Periodicity: $T_{m}^{(a)}\left(u+2\left(h^{\vee}+\ell\right)\right)=T_{m}^{(a)}(u)$.

Proof. It suffices to prove the half-periodicity (1). First consider the case $X_{N}^{(\kappa)} \neq D_{r+1}^{(2)}(r+1$ : even $)$ and $D_{4}^{(3)}$. Then we have $\sigma=\omega$ and $\kappa=2$. Thanks to the first half of Proposition 9.19, it is equivalent to showing that $\hat{T}_{m}^{(a)}(u+$ $\left.h^{\vee}+\ell\right)=\hat{T}_{\ell-m}^{(a)}(u+\Omega)$ for $a \in I_{\sigma}$ in $\mathcal{T}_{\ell}\left(X_{N}\right) / \mathcal{J}_{\ell}^{\sigma}$. Both the left and the right sides coincide with $\hat{T}_{\ell-m}^{(\sigma(a))}(u)$ due to the half-periodicity of $X_{N}$ and (9.11), respectively.

Next consider the remaining case $X_{N}^{(\kappa)}=D_{r+1}^{(2)}(r+1$ : even $)$ or $D_{4}^{(3)}$. Then we have $\omega=\mathrm{id}$. By the same reason as before, we show that $\hat{T}_{m}^{(a)}\left(u+h^{\vee}+\right.$ $\ell)=\hat{T}_{\ell-m}^{(a)}(u)$ for $a \in I_{\sigma}$ in $\mathcal{T}_{\ell}\left(X_{N}\right) / \mathcal{J}_{\ell}^{\sigma}$. Again, this is guaranteed by the half-periodicity of $X_{N}$.

Similarly, the periodicity of $y_{\ell}\left(X_{N}^{(\kappa)}\right)$ reduces to that of $y_{\ell}\left(X_{N}\right)$. Recall that Conjecture 3.12 has been proved for $y_{\ell}\left(X_{N}\right)$, except for the halfperiodicity for $D_{N}$ and $E_{6}$.

Theorem 9.21. Suppose that Conjecture 3.12(1) is also true for $D_{N}$ and $E_{6}$. Then, the following relations hold in $y_{\ell}\left(X_{N}^{(\kappa)}\right)$ : 
(1) Half-periodicity:

$$
Y_{m}^{(a)}\left(u+h^{\vee}+\ell\right)= \begin{cases}Y_{\ell-m}^{(a)}(u) & \text { if } X_{N}^{(\kappa)}=D_{r+1}^{(2)}\left(r+1 \text { : even) or } D_{4}^{(3)}\right. \\ Y_{\ell-m}^{(a)}(u+\Omega) & \text { otherwise. }\end{cases}
$$

(2) Periodicity: $Y_{m}^{(a)}\left(u+2\left(h^{\vee}+\ell\right)\right)=Y_{m}^{(a)}(u)$.

REMARK 9.22. By formally setting $\Omega=0$ (i.e., $\hbar \rightarrow \infty ; q \rightarrow 0, \infty$ ) or by imposing a further relation $Y_{m}^{(a)}(u)=Y_{m}^{(a)}(u+\Omega)$ in $y_{\ell}\left(X_{N}^{(\kappa)}\right)$ for $X_{N}^{(\kappa)}=$ $A_{2 r-1}^{(2)}\left(\right.$ resp. $\left.D_{r+1}^{(2)}, E_{6}^{(2)}, D_{4}^{(3)}\right)$, one gets the Y-system of the form

$$
Y_{m}^{(a)}(u-1) Y_{m}^{(a)}(u+1)=\frac{\prod_{b \in I}\left(1+Y_{m}^{(b)}(u)\right)^{2 \delta_{a b}-C_{a b}}}{\left(1+Y_{m-1}^{(a)}(u)^{-1}\right)\left(1+Y_{m+1}^{(a)}(u)^{-1}\right)} .
$$

Here, $C_{a b}=2\left(\alpha_{a}, \alpha_{b}\right) /\left(\alpha_{a}, \alpha_{a}\right)$ is the Cartan matrix for $B_{r}$ (resp. $C_{r}, F_{4}$, $G_{2}$ ) with the enumeration $I$ in Figure 1 . This is the Y-system for nonsimply laced $X_{r}$ considered in [FZ3], [Kel2]. It was proved, by [FZ3] for $\ell=2$ and by [Kel2] for any $\ell \geq 2$, that the system (9.36) has the full-period $2(h+\ell)$, where $h$ is the Coxeter number of $B_{r}$ (resp. $C_{r}, F_{4}, G_{2}$ ). This completely agrees with Theorem 9.21, since the dual Coxeter number $h^{\vee}$ of $A_{2 r-1}^{(2)}$ (resp. $D_{r+1}^{(2)}, E_{6}^{(2)}, D_{4}^{(3)}$ ) equals the Coxeter number $h$ of $B_{r}$ (resp. $C_{r}, F_{4}, G_{2}$ ). By the same token, one can obtain from $\mathcal{T}_{\ell}\left(X_{N}^{(\kappa)}\right)$ the T-system of the form

$$
T_{m}^{(a)}(u-1) T_{m}^{(a)}(u+1)=T_{m-1}^{(a)}(u) T_{m+1}^{(a)}(u)+\prod_{b \in I} T_{m}^{(b)}(u)^{2 \delta_{a b}-C_{a b}},
$$

whose periodicity is the same as (9.36).

\subsection{Periodicities of restricted T- and $\mathrm{Y}$-systems at level 0}

Here we introduce the level 0 restricted T-system $\mathbb{T}_{0}\left(X_{N}^{(\kappa)}\right)$ and T-group $\mathcal{T}_{0}\left(X_{N}^{(\kappa)}\right)$ of type $X_{N}^{(\kappa)}$ in a manner similar to Section 3 . The analogous construction of the Y-system and Y-group leads to exactly the same objects. Thus one should understand $\mathcal{T}_{0}\left(X_{N}^{(\kappa)}\right) \simeq y_{0}\left(X_{N}^{(\kappa)}\right)$ by $T^{(a)}(u) \leftrightarrow Y^{(a)}(u)$ in the sequel. Such a coincidence has been already encountered in the untwisted case between $\mathbb{T}_{0}\left(X_{r}\right)$ (8.4) and $\mathbb{Y}_{0}\left(X_{r}\right)$ (8.9) for simply laced $X_{r}$.

Definition 9.23. The level 0 restricted T-system $\mathbb{T}_{0}\left(X_{N}^{(\kappa)}\right)$ of type $X_{N}^{(\kappa)}$ is the following system of relations for a family of variables $T=\left\{T^{(a)}(u) \mid\right.$ $\left.a \in I_{\sigma}, u \in \mathbb{C}_{\kappa_{a} \hbar}\right\}$, where $\Omega=2 \pi \sqrt{-1} / \kappa \hbar$, and $T^{(0)}(u)=1$ if they occur in the right-hand sides in the relations. 
For $X_{N}^{(\kappa)}=A_{2 r-1}^{(2)}$,

$$
\begin{aligned}
& T^{(a)}(u-1) T^{(a)}(u+1)=T^{(a-1)}(u) T^{(a+1)}(u) \quad(1 \leq a \leq r-1), \\
& T^{(r)}(u-1) T^{(r)}(u+1)=T^{(r-1)}(u) T^{(r-1)}(u+\Omega) .
\end{aligned}
$$

For $X_{N}^{(\kappa)}=A_{2 r}^{(2)}$

$$
\begin{aligned}
& T^{(a)}(u-1) T^{(a)}(u+1)=T^{(a-1)}(u) T^{(a+1)}(u) \quad(1 \leq a \leq r-1), \\
& T^{(r)}(u-1) T^{(r)}(u+1)=T^{(r-1)}(u) T^{(r)}(u+\Omega) .
\end{aligned}
$$

For $X_{N}^{(\kappa)}=D_{r+1}^{(2)}$,

$$
\begin{aligned}
T^{(a)}(u-1) T^{(a)}(u+1) & =T^{(a-1)}(u) T^{(a+1)}(u) \quad(1 \leq a \leq r-2), \\
T^{(r-1)}(u-1) T^{(r-1)}(u+1) & =T^{(r-2)}(u) T^{(r)}(u) T^{(r)}(u+\Omega), \\
T^{(r)}(u-1) T^{(r)}(u+1) & =T^{(r-1)}(u) .
\end{aligned}
$$

For $X_{N}^{(\kappa)}=E_{6}^{(2)}$

$$
\begin{aligned}
& T^{(1)}(u-1) T^{(1)}(u+1)=T^{(2)}(u), \\
& T^{(2)}(u-1) T^{(2)}(u+1)=T^{(1)}(u) T^{(3)}(u), \\
& T^{(3)}(u-1) T^{(3)}(u+1)=T^{(2)}(u) T^{(2)}(u+\Omega) T^{(4)}(u), \\
& T^{(4)}(u-1) T^{(4)}(u+1)=T^{(3)}(u) .
\end{aligned}
$$

For $X_{N}^{(\kappa)}=D_{4}^{(3)}$

$$
\begin{aligned}
& T^{(1)}(u-1) T^{(1)}(u+1)=T^{(2)}(u), \\
& T^{(2)}(u-1) T^{(2)}(u+1)=T^{(1)}(u) T^{(1)}(u-\Omega) T^{(1)}(u+\Omega) .
\end{aligned}
$$

Definition 9.24. The level 0 restricted T-group $\mathcal{T}_{0}\left(X_{N}^{(\kappa)}\right)$ of type $X_{N}^{(\kappa)}$ is the abelian group with generators $T^{(a)}(u)\left(a \in I_{\sigma}, u \in \mathbb{C}_{\kappa_{a} \hbar}\right)$ and the relations $\mathbb{T}_{0}\left(X_{N}^{(\kappa)}\right)$.

REMARK 9.25. $\mathbb{T}_{0}\left(X_{N}^{(\kappa)}\right)$ is obtained from the unrestricted T-system $\mathbb{T}\left(X_{N}^{(\kappa)}\right)(9.5)-(9.9)$ by setting $T_{m}^{(a)}(u)=T^{(a)}(u)$ if $m=0$ and $T_{m}^{(a)}(u)=0$ otherwise. 
Let $\left\{\hat{T}^{(a)}(u) \mid a \in I, u \in \mathbb{C}_{\hbar}\right\}$ be the set of generators of $\mathcal{T}_{0}\left(X_{N}\right)$. Let $\mathcal{g}_{0}^{\sigma}$ be the subgroup of $\mathcal{T}_{0}\left(X_{N}\right)$ generated by

$$
\hat{T}^{(a)}(u) \hat{T}^{(\sigma(a))}(u+\Omega)^{-1} \quad\left(a \in I, u \in \mathbb{C}_{\hbar}\right) .
$$

Then one can choose a generating set of $\mathcal{T}_{0}\left(X_{N}\right) / \mathcal{J}_{0}^{\sigma}$ as $\left\{\hat{T}^{(a)}(u)^{ \pm 1} \mid a \in\right.$ $I_{\sigma}, u \in \mathbb{C}_{\left.\kappa_{a} \hbar\right\}}$.

Proposition 9.26. There is a group isomorphism

$$
\begin{aligned}
\mathcal{T}_{0}\left(X_{N}\right) / \mathcal{J}_{0}^{\sigma} & \rightarrow \mathcal{T}_{0}\left(X_{N}^{(\kappa)}\right), \\
\hat{T}^{(a)}(u) & \mapsto T^{(a)}(u) \quad\left(a \in I_{\sigma}\right) .
\end{aligned}
$$

Proof. It is easy to check that the relations of the both rings are identical under the correspondence.

THEOREM 9.27. The following relations hold in $\mathcal{T}_{0}\left(X_{N}^{(\kappa)}\right)$ :

(1) Half-periodicity:

$$
T^{(a)}\left(u+h^{\vee}\right)= \begin{cases}T^{(a)}(u)^{-1} & \text { if } X_{N}^{(\kappa)}=D_{r+1}^{(2)}\left(r+1 \text { : even) or } D_{4}^{(3)},\right. \\ T^{(a)}(u+\Omega)^{-1} & \text { otherwise. }\end{cases}
$$

(2) Periodicity: $T^{(a)}\left(u+2 h^{\vee}\right)=T^{(a)}(u)$.

Proof. It suffices to prove the half-periodicity (1). First consider the case $X_{N}^{(\kappa)} \neq D_{r+1}^{(2)}(r+1$ : even $)$ and $D_{4}^{(3)}$. Then we have $\sigma=\omega$ and $\kappa=2$. Thanks to Proposition 9.26, it is equivalent to showing $\hat{T}^{(a)}\left(u+h^{\vee}\right)=\hat{T}^{(a)}(u+\Omega)^{-1}$ for $a \in I_{\sigma}$ in $\mathcal{T}_{0}\left(X_{N}\right) / \mathcal{J}_{0}^{\sigma}$. This equality is verified by Theorem 8.8 and (9.43).

Next consider the remaining case $X_{N}^{(\kappa)}=D_{r+1}^{(2)}(r+1$ : even $)$ or $D_{4}^{(3)}$. Then we have $\omega=\mathrm{id}$. By the same reason as before, we show that $\hat{T}^{(a)}\left(u+h^{\vee}\right)=$ $\hat{T}^{(a)}(u)$ for $a \in I_{\sigma}$ in $\mathcal{T}_{0}\left(X_{N}\right) / \mathcal{J}_{0}^{\sigma}$. Again, this is guaranteed by Theorem 8.8.

\section{$\S 10$. Remark on the periodicity of $q$-characters}

We conclude the paper with a remark on a formal correspondence between the periodicity of the T-system and the $q$-characters of $U_{q}(\hat{\mathfrak{g}})$ at roots of unity.

Recall that $\mathcal{T}^{\circ}\left(X_{r}\right) \simeq \operatorname{Ch} U_{q}(\hat{\mathfrak{g}})$ by Corollary 2.9. Let

$$
\pi_{\ell}: \mathcal{T}^{\circ}\left(X_{r}\right) \rightarrow \mathcal{T}_{\ell}^{\circ}\left(X_{r}\right)
$$


be the surjective ring homomorphism in (3.1). One can easily see that $T_{t_{a} \ell}^{(a)}(u)-1$ and $T_{t_{a} \ell+1}^{(a)}(u)$ are in Ker $\pi_{\ell}$. Correspondingly, we define the level $\ell$ restricted $q$-character ring $\mathrm{Ch}_{\ell} U_{q}(\hat{\mathfrak{g}})$ of $U_{q}(\hat{\mathfrak{g}})$ by

$$
\mathrm{Ch}_{\ell} U_{q}(\hat{\mathfrak{g}})=\mathrm{Ch} U_{q}(\hat{\mathfrak{g}}) / I_{\ell}
$$

where $I_{\ell}$ is the ideal of $\mathrm{Ch} U_{q}(\hat{\mathfrak{g}})$ corresponding to $\operatorname{Ker} \pi_{\ell}$ under the isomorphism $\mathcal{T}^{\circ}\left(X_{r}\right) \simeq \mathrm{Ch} U_{q}(\hat{\mathfrak{g}})$. By construction, we have an isomorphism,

$$
\begin{aligned}
\mathrm{Ch}_{\ell} U_{q}(\hat{\mathfrak{g}}) & \stackrel{\sim}{\rightarrow} \mathcal{T}_{\ell}^{\circ}\left(X_{r}\right), \\
\chi_{q}\left(W_{m}^{(a)}(u)\right) & \mapsto T_{m}^{(a)}(u) \quad\left(1 \leq m \leq t_{a} \ell-1\right),
\end{aligned}
$$

and the periodicity of $\mathcal{T}_{\ell}\left(X_{r}\right)$ in Conjecture 3.11 is rephrased as the following periodicity of the $q$-characters. For $1 \leq m \leq t_{a} \ell-1$,

$$
\chi_{q}\left(W_{m}^{(a)}\left(u+2\left(h^{\vee}+\ell\right)\right)\right) \equiv \chi_{q}\left(W_{m}^{(a)}(u)\right) \quad \bmod I_{\ell}
$$

In view of (2.12), this implies (but does not directly require) $q^{2 t\left(h^{\vee}+\ell\right)}=1$, which is natural, as mentioned in the end of Section 3.3. Let us make this implication, still formal, but more manifest in the form of a conjecture. Recall that $\operatorname{Ch} U_{q}(\hat{\mathfrak{g}})$ is a subring of the ring $\mathbb{Z}\left[Y_{a, q^{t u}}^{ \pm 1}\right]_{a \in I, u \in \mathbb{C}_{t \hbar}}$. Let $I_{\ell}^{\prime}$ be the ideal of $\mathbb{Z}\left[Y_{a, q^{t u}}^{ \pm 1}\right]_{a \in I, u \in \mathbb{C}_{t \hbar}}$ generated by $I_{\ell}$.

Conjecture 10.1. The following equality holds in $\mathbb{Z}\left[Y_{a, q^{t u}}^{ \pm 1}\right]_{a \in I, u \in \mathbb{C}_{t \hbar}}$ :

$$
Y_{a, q^{t u+2 t\left(h^{\vee}+\ell\right)}} \equiv Y_{a, q^{t u}} \quad \bmod I_{\ell}^{\prime}
$$

ExAmple 10.2. (1) $X_{r}=A_{1}$. We set $W_{m}(u)=W_{m}^{(1)}(u)$ and $Y_{q^{u}}=Y_{1, q^{u}}$, for simplicity. Recall that [FR, Section 4.1]

$$
\begin{aligned}
\chi_{q}\left(W_{m}(u)\right) & =\left(\prod_{j=1}^{m} Y_{q^{u+m+1-2 j}}\right) \sum_{i=0}^{m} \prod_{j=1}^{i} A_{q^{u+m+2-2 j}}^{-1}, \\
A_{q^{u}} & :=Y_{q^{u-1}} Y_{q^{u+1}} .
\end{aligned}
$$

Thus, we have

$$
\begin{aligned}
\chi_{q}\left(W_{\ell+1}(u)\right)= & Y_{q^{u-\ell}} \chi_{q}\left(W_{\ell}(u+1)\right) \\
& +Y_{q^{u-\ell+2}}^{-1} Y_{q^{u-\ell+4}}^{-1} \cdots Y_{q^{u+\ell+2}}^{-1} .
\end{aligned}
$$


Meanwhile, $\chi_{q}\left(W_{\ell}(u)\right) \equiv 1$ and $\chi_{q}\left(W_{\ell+1}(u)\right) \equiv 0$. Therefore,

$$
Y_{q^{u-\ell}} Y_{q^{u-\ell+2}} \cdots Y_{q^{u+\ell+2}} \equiv-1,
$$

from which $Y_{q^{u+2(2+\ell)}} \equiv Y_{q^{u}}$ follows.

(2) $X_{r}=A_{r}(r \geq 2)$. We remark that, in addition to $T_{\ell}^{(a)}(u)-1$ and $T_{\ell+1}^{(a)}(u), T_{\ell+2}^{(1)}(u), \ldots, T_{\ell+r}^{(1)}(u)$ are also in Ker $\pi_{\ell}$. Then, generalizing the argument of $(1)$, one can show that

$$
Y_{a, q^{u}} Y_{a, q^{u+2}} \cdots Y_{a, q^{u+2(r+\ell)}} \equiv(-1)^{a r} \bmod I_{\ell}^{\prime}
$$

from which $Y_{a, q^{u+2(r+1+\ell)}} \equiv Y_{a, q^{u}}$ follows. More detail is given in Appendix B.

It is important to establish a precise relation between the ring $\mathrm{Ch}_{\ell} U_{q}(\hat{\mathfrak{g}})$ and the ring $\operatorname{Rep} U_{\epsilon}^{\text {res }}(\hat{\mathfrak{g}})$ of [FM2] for a primitive $2 t\left(h^{\vee}+\ell\right)$ th root of unity $\epsilon$. A similar remark is applicable to the twisted quantum affine algebras as well.

\section{Appendix A. Q-systems and $\operatorname{Rep} U_{q}(\mathfrak{g})$}

\section{A.1. Q-systems for untwisted case}

Here we present results parallel to Theorem 2.8 and Corollary 2.9 for the Q-system and $\operatorname{Rep} U_{q}(\mathfrak{g})$. For a Dynkin diagram $X_{r}$, let $I$ be as in Section 2.

The following system was introduced by [Ki], [KR].

Definition A.1. The unrestricted Q-system $\mathbb{Q}\left(X_{r}\right)$ of type $X_{r}$ is the following system of relations for a family of variables $Q=\left\{Q_{m}^{(a)} \mid a \in I, m \in\right.$ $\mathbb{N}\}$, where $Q_{m}^{(0)}(u)=Q_{0}^{(a)}(u)=1$ if they occur in the right-hand sides in the following relations.

For simply laced $X_{r}$,

$$
\left(Q_{m}^{(a)}\right)^{2}=Q_{m-1}^{(a)} Q_{m+1}^{(a)}+\prod_{b \in I: C_{a b}=-1} Q_{m}^{(b)} .
$$

For $X_{r}=B_{r}$,

$$
\begin{aligned}
\left(Q_{m}^{(a)}\right)^{2} & =Q_{m-1}^{(a)} Q_{m+1}^{(a)}+Q_{m}^{(a-1)} Q_{m}^{(a+1)} \quad(1 \leq a \leq r-2), \\
\left(Q_{m}^{(r-1)}\right)^{2} & =Q_{m-1}^{(r-1)} Q_{m+1}^{(r-1)}+Q_{m}^{(r-2)} Q_{2 m}^{(r)}, \\
\left(Q_{2 m}^{(r)}\right)^{2} & =Q_{2 m-1}^{(r)}(u) Q_{2 m+1}^{(r)}(u)+\left(Q_{m}^{(r-1)}\right)^{2}, \\
\left(Q_{2 m+1}^{(r)}\right)^{2} & =Q_{2 m}^{(r)} Q_{2 m+2}^{(r)}+Q_{m}^{(r-1)} Q_{m+1}^{(r-1)} .
\end{aligned}
$$


For $X_{r}=C_{r}$,

$$
\begin{aligned}
\left(Q_{m}^{(a)}\right)^{2} & =Q_{m-1}^{(a)} Q_{m+1}^{(a)}+Q_{m}^{(a-1)} Q_{m}^{(a+1)} \quad(1 \leq a \leq r-2), \\
\left(Q_{2 m}^{(r-1)}\right)^{2} & =Q_{2 m-1}^{(r-1)} Q_{2 m+1}^{(r-1)}+Q_{2 m}^{(r-2)}\left(Q_{m}^{(r)}\right)^{2} \\
\left(Q_{2 m+1}^{(r-1)}\right)^{2} & =Q_{2 m}^{(r-1)} Q_{2 m+2}^{(r-1)}+Q_{2 m+1}^{(r-2)} Q_{m}^{(r)} Q_{m+1}^{(r)}, \\
\left(Q_{m}^{(r)}\right)^{2} & =Q_{m-1}^{(r)} Q_{m+1}^{(r)}+Q_{2 m}^{(r-1)}
\end{aligned}
$$

For $X_{r}=F_{4}$,

$$
\begin{aligned}
\left(Q_{m}^{(1)}\right)^{2} & =Q_{m-1}^{(1)} Q_{m+1}^{(1)}+Q_{m}^{(2)} \\
\left(Q_{m}^{(2)}\right)^{2} & =Q_{m-1}^{(2)} Q_{m+1}^{(2)}+Q_{m}^{(1)} Q_{2 m}^{(3)}, \\
\left(Q_{2 m}^{(3)}\right)^{2} & =Q_{2 m-1}^{(3)} Q_{2 m+1}^{(3)}+\left(Q_{m}^{(2)}\right)^{2} Q_{2 m}^{(4)}, \\
\left(Q_{2 m+1}^{(3)}\right)^{2} & =Q_{2 m}^{(3)} Q_{2 m+2}^{(3)}+Q_{m}^{(2)} Q_{m+1}^{(2)} Q_{2 m+1}^{(4)} \\
\left(Q_{m}^{(4)}\right)^{2} & =Q_{m-1}^{(4)} Q_{m+1}^{(4)}+Q_{m}^{(3)} .
\end{aligned}
$$

For $X_{r}=G_{2}$,

$$
\begin{aligned}
\left(Q_{m}^{(1)}\right)^{2} & =Q_{m-1}^{(1)} Q_{m+1}^{(1)}+Q_{3 m}^{(2)}, \\
\left(Q_{3 m}^{(2)}\right)^{2} & =Q_{3 m-1}^{(2)} Q_{3 m+1}^{(2)}+\left(Q_{m}^{(1)}\right)^{3}, \\
\left(Q_{3 m+1}^{(2)}\right)^{2} & =Q_{3 m}^{(2)} Q_{3 m+2}^{(2)}+\left(Q_{m}^{(1)}\right)^{2} Q_{m+1}^{(1)}, \\
\left(Q_{3 m+2}^{(2)}\right)^{2} & =Q_{3 m+1}^{(2)} Q_{3 m+3}^{(2)}+Q_{m}^{(1)}(u)\left(Q_{m+1}^{(1)}\right)^{2} .
\end{aligned}
$$

Definition A.2. The unrestricted Q-algebra $\mathcal{Q}\left(X_{r}\right)$ of type $X_{r}$ is the ring with generators $Q_{m}^{(a)} \pm 1 \quad(a \in I, m \in \mathbb{N})$ and the relations $\mathbb{Q}\left(X_{r}\right)$. Also, we define the ring $\mathcal{Q}^{\circ}\left(X_{r}\right)$ as the subring of $\mathcal{Q}\left(X_{r}\right)$ generated by $Q_{m}^{(a)}(a \in$ $I, m \in \mathbb{N})$.

The system $\mathbb{Q}\left(X_{r}\right)$ is obtained by $\mathbb{T}\left(X_{r}\right)$ by the reduction of the spectral parameter $u$. One can also define the level $\ell$ restricted $Q$-system $\mathbb{Q}_{\ell}\left(X_{r}\right)$ by the reduction of $\mathbb{T}_{\ell}\left(X_{r}\right)$ [KNS1]. The system $\mathbb{Q}_{\ell}\left(X_{r}\right)$ plays the central role in the dilogarithm identities for the central charges of conformal field theories (e.g., [Ki], [Ku], [KN], [KNS1], [KNS2], [RTV], [FS], [GT], etc.).

Let $\mathfrak{g}$ be the complex simple Lie algebra of type $X_{r}$, and let $U_{q}(\mathfrak{g})$ be the quantized universal enveloping algebra of $\mathfrak{g}$. Then, $U_{q}(\mathfrak{g})$ is a subalgebra of 
the untwisted quantum affine algebra $U_{q}(\hat{\mathfrak{g}})$. Let $\chi$ be the character map of $U_{q}(\mathfrak{g})$; it is an injective ring homomorphism $\chi: \operatorname{Rep} U_{q}(\mathfrak{g}) \rightarrow \mathbb{Z}\left[y_{a}^{ \pm 1}\right]_{a \in I}$, where we follow the notation of $[\mathrm{FR}]$. The character ring $\mathrm{Ch} U_{q}(\mathfrak{g})$ of $U_{q}(\mathfrak{g})$ is defined to be $\operatorname{Im} \chi$. Thus, $\mathrm{Ch} U_{q}(\mathfrak{g})$ is an integral domain and isomorphic to $\operatorname{Rep} U_{q}(\mathfrak{g})$. Let $\operatorname{Res}: \operatorname{Rep} U_{q}(\hat{\mathfrak{g}}) \rightarrow \operatorname{Rep} U_{q}(\mathfrak{g})$ be the restriction homomorphism induced from the inclusion $U_{q}(\mathfrak{g}) \rightarrow U_{q}(\hat{\mathfrak{g}})$.

A Kirillov-Reshetikhin module $W_{m}^{(a)}(a \in I, m \in \mathbb{N})$ of $U_{q}(\mathfrak{g})$ is the (not necessarily irreducible) $U_{q}(\mathfrak{g})$-module defined by $W_{m}^{(a)}:=\operatorname{Res}\left(W_{m}^{(a)}(u)\right)$, where $W_{m}^{(a)}(u)$ is a Kirillov-Reshetikhin module of $U_{q}(\hat{\mathfrak{g}})$. We remark that $W_{m}^{(a)}$ is independent of $u$.

In the same way as $(2.9)$, we define $S_{a m}(Q) \in \mathbb{Z}[Q](a \in I, m \in \mathbb{N})$, so that all the relations in $\mathbb{Q}\left(X_{r}\right)$ are written in the form $S_{a m}(Q)=0$. Let $I\left(\mathbb{Q}\left(X_{r}\right)\right)$ be the ideal of $\mathbb{Z}[Q]$ generated by $S_{a m}(Q)$.

From Theorem 2.8, we obtain the following.

Theorem A.3. Let $\widetilde{Q}=\left\{\widetilde{Q}_{m}^{(a)}:=\chi\left(W_{m}^{(a)}\right) \mid a \in I, m \in \mathbb{N}\right\}$ be the family of the characters of the Kirillov-Reshetikhin modules of $U_{q}(\mathfrak{g})$. Then, we have the following.

(1) The family $\widetilde{Q}$ generates the ring $\operatorname{Ch} U_{q}(\mathfrak{g})$.

(2) ([N3], [Her1]) The family $\widetilde{Q}$ satisfies the Q-system $\mathbb{Q}\left(X_{r}\right)$ in $\operatorname{Ch} U_{q}(\mathfrak{g})$ (by replacing $Q_{m}^{(a)}$ in $\mathbb{Q}\left(X_{r}\right)$ with $\left.\widetilde{Q}_{m}^{(a)}\right)$.

(3) For any $P(Q) \in \mathbb{Z}[Q]$, the relation $P(\widetilde{Q})=0$ holds in $\operatorname{Ch} U_{q}(\mathfrak{g})$ if and only if there is a nonzero monomial $M(Q) \in \mathbb{Z}[Q]$ such that $M(Q) P(Q) \in$ $I\left(\mathbb{Q}\left(X_{r}\right)\right)$.

Corollary A.4. The ring $\mathcal{Q}^{\circ}\left(X_{r}\right)$ is isomorphic to $\operatorname{Rep} U_{q}(\mathfrak{g})$ by the correspondence $Q_{m}^{(a)} \mapsto W_{m}^{(a)}$.

By taking $q \rightarrow 1$, one can also obtain analogous results for Rep $\mathfrak{g}$.

\section{A.2. Q-systems for twisted case}

Here we present results parallel to Theorem 9.8 and Corollary 9.9 for the Q-system and $\operatorname{Rep} U_{q}\left(\mathfrak{g}^{\sigma}\right)$. For a pair $\left(X_{N}, \kappa\right)=\left(A_{N}, 2\right),\left(D_{N}, 2\right),\left(E_{6}, 2\right)$, or $\left(D_{4}, 3\right)$, let $I_{\sigma}$ be as in Section 9 .

The following system was introduced by [HKOTT].

Definition A.5. The unrestricted Q-system $\mathbb{Q}\left(X_{N}^{(\kappa)}\right)$ of type $X_{N}^{(\kappa)}$ is the following system of relations for a family of variables $Q=\left\{Q_{m}^{(a)} \mid a \in I_{\sigma}, m \in\right.$ 
$\mathbb{N}\}$, where $Q_{m}^{(0)}(u)=Q_{0}^{(a)}(u)=1$ if they occur in the right-hand sides in the relations.

For $X_{N}^{(\kappa)}=A_{2 r-1}^{(2)}$,

$$
\begin{aligned}
& \left(Q_{m}^{(a)}\right)^{2}=Q_{m-1}^{(a)} Q_{m+1}^{(a)}+Q_{m}^{(a-1)} Q_{m}^{(a+1)} \quad(1 \leq a \leq r-1) \\
& \left(Q_{m}^{(r)}\right)^{2}=Q_{m-1}^{(r)} Q_{m+1}^{(r)}+\left(Q_{m}^{(r-1)}\right)^{2} .
\end{aligned}
$$

For $X_{N}^{(\kappa)}=A_{2 r}^{(2)}$

$$
\begin{aligned}
& \left(Q_{m}^{(a)}\right)^{2}=Q_{m-1}^{(a)} Q_{m+1}^{(a)}+Q_{m}^{(a-1)} Q_{m}^{(a+1)} \quad(1 \leq a \leq r-1) \\
& \left(Q_{m}^{(r)}\right)^{2}=Q_{m-1}^{(r)} Q_{m+1}^{(r)}+Q_{m}^{(r-1)} Q_{m}^{(r)} .
\end{aligned}
$$

For $X_{N}^{(\kappa)}=D_{r+1}^{(2)}$,

$$
\begin{aligned}
\left(Q_{m}^{(a)}\right)^{2} & =Q_{m-1}^{(a)} Q_{m+1}^{(a)}+Q_{m}^{(a-1)} Q_{m}^{(a+1)} \quad(1 \leq a \leq r-2) \\
\left(Q_{m}^{(r-1)}\right)^{2} & =Q_{m-1}^{(r-1)} Q_{m+1}^{(r-1)}+Q_{m}^{(r-2)}\left(Q_{m}^{(r)}\right)^{2} \\
\left(Q_{m}^{(r)}\right)^{2} & =Q_{m-1}^{(r)} Q_{m+1}^{(r)}+Q_{m}^{(r-1)}
\end{aligned}
$$

For $X_{N}^{(\kappa)}=E_{6}^{(2)}$

$$
\begin{aligned}
& \left(Q_{m}^{(1)}\right)^{2}=Q_{m-1}^{(1)} Q_{m+1}^{(1)}+Q_{m}^{(2)} \\
& \left(Q_{m}^{(2)}\right)^{2}=Q_{m-1}^{(2)} Q_{m+1}^{(2)}+Q_{m}^{(1)} Q_{m}^{(3)} \\
& \left(Q_{m}^{(3)}\right)^{2}=Q_{m-1}^{(3)} Q_{m+1}^{(3)}+\left(Q_{m}^{(2)}\right)^{2} Q_{m}^{(4)} \\
& \left(Q_{m}^{(4)}\right)^{2}=Q_{m-1}^{(4)} Q_{m+1}^{(4)}+Q_{m}^{(3)}
\end{aligned}
$$

For $X_{N}^{(\kappa)}=D_{4}^{(3)}$

$$
\begin{aligned}
& \left(Q_{m}^{(1)}\right)^{2}=Q_{m-1}^{(1)} Q_{m+1}^{(1)}+Q_{m}^{(2)}, \\
& \left(Q_{m}^{(2)}\right)^{2}=Q_{m-1}^{(2)} Q_{m+1}^{(2)}+\left(Q_{m}^{(1)}\right)^{3} .
\end{aligned}
$$

Definition A.6. The unrestricted $Q$-algebra $\mathcal{Q}\left(X_{N}^{(\kappa)}\right)$ of type $X_{N}^{(\kappa)}$ is the ring with generators $Q_{m}^{(a)} \pm 1\left(a \in I_{\sigma}, m \in \mathbb{N}\right)$ and the relations $\mathbb{Q}\left(X_{N}^{(\kappa)}\right)$. Also, we define the ring $\mathcal{Q}^{\circ}\left(X_{N}^{(\kappa)}\right)$ as the subring of $\mathcal{Q}\left(X_{N}^{(\kappa)}\right)$ generated by $Q_{m}^{(a)}$ $\left(a \in I_{\sigma}, m \in \mathbb{N}\right)$. 
The system $\mathbb{Q}\left(X_{N}^{(\kappa)}\right)$ is obtained by $\mathbb{T}\left(X_{N}^{(\kappa)}\right)$ by the reduction of the spectral parameter $u$. One can also define the level $\ell$ restricted $Q$-system $\mathbb{Q}_{\ell}\left(X_{N}^{(\kappa)}\right)$ by the reduction of $\mathbb{T}_{\ell}\left(X_{N}^{(\kappa)}\right)$.

Let $X_{N}^{\sigma}$ be the subdiagram of $X_{N}^{(\kappa)}$ obtained by removing the zeroth node. Explicitly,

\begin{tabular}{c|cccccc}
$X_{N}^{(\kappa)}$ & $A_{2 r-1}^{(2)}$ & $A_{2}^{(2)}$ & $A_{2 r}^{(2)}$ & $D_{r+1}^{(2)}$ & $E_{6}^{(2)}$ & $D_{4}^{(3)}$ \\
\hline$X_{N}^{\sigma}$ & $C_{r}$ & $A_{1}$ & $B_{r}$ & $B_{r}$ & $F_{4}$ & $G_{2}$
\end{tabular}

Let $\mathfrak{g}^{\sigma}$ be the complex simple Lie algebra of type $X_{N}^{\sigma}$, and let $U_{q}\left(\mathfrak{g}^{\sigma}\right)$ be the quantized universal enveloping algebra of $\mathfrak{g}^{\sigma}$. Then, $U_{q}\left(\mathfrak{g}^{\sigma}\right)$ is a subalgebra of the twisted quantum affine algebra $U_{q}\left(\hat{\mathfrak{g}}^{\sigma}\right)$ of type $X_{N}^{(\kappa)}$ [Her2]. Let $\operatorname{Res}^{\sigma}: \operatorname{Rep} U_{q}\left(\hat{\mathfrak{g}}^{\sigma}\right) \rightarrow \operatorname{Rep} U_{q}\left(\mathfrak{g}^{\sigma}\right)$ be the restriction homomorphism induced from the inclusion $U_{q}\left(\mathfrak{g}^{\sigma}\right) \rightarrow U_{q}\left(\hat{\mathfrak{g}}^{\sigma}\right)$.

We define the (not necessarily irreducible) $U_{q}\left(\mathfrak{g}^{\sigma}\right)$-module $\dot{W}_{m}^{(a)}\left(a \in I_{\sigma}\right.$, $m \in \mathbb{N})$ by $\dot{W}_{m}^{(a)}:=\operatorname{Res}^{\sigma}\left(W_{m}^{(a)}(u)\right)$, where $W_{m}^{(a)}(u)$ is a Kirillov-Reshetikhin module of $U_{q}\left(\hat{\mathfrak{g}}^{\sigma}\right)$. We remark that $\dot{W}_{m}^{(a)}$ is independent of $u$.

In the same way as $(2.9)$, we define $S_{a m}(Q) \in \mathbb{Z}[Q](a \in I, m \in \mathbb{N})$, so that all the relations in $\mathbb{Q}\left(X_{N}^{(\kappa)}\right)$ are written in the form $S_{a m}(Q)=0$. Let $I\left(\mathbb{Q}\left(X_{N}^{(\kappa)}\right)\right)$ be the ideal of $\mathbb{Z}[Q]$ generated by $S_{a m}(Q)$ 's.

As Theorem 9.8, we obtain the following.

TheOrem A.7. Let $\widetilde{Q}=\left\{\widetilde{Q}_{m}^{(a)}:=\chi\left(\dot{W}_{m}^{(a)}\right) \mid a \in I_{\sigma}, m \in \mathbb{N}\right\}$ be the family of the characters of $\dot{W}_{m}^{(a)}(u)$ 's. Then, we have the following:

(1) The family $\widetilde{Q}$ generates the ring $\mathrm{Ch} U_{q}\left(\mathfrak{g}^{\sigma}\right)$.

(2) ([Her2]) The family $\widetilde{Q}$ satisfies the $Q$-system $\mathbb{Q}\left(X_{N}^{(\kappa)}\right)$ in $\operatorname{Ch} U_{q}\left(\mathfrak{g}^{\sigma}\right)$ (by replacing $Q_{m}^{(a)}$ in $\mathbb{Q}\left(X_{N}^{(\kappa)}\right)$ with $\left.\widetilde{Q}_{m}^{(a)}\right)$.

(3) For any $P(Q) \in \mathbb{Z}[Q]$, the relation $P(\widetilde{Q})=0$ holds in $\operatorname{Ch} U_{q}\left(\mathfrak{g}^{\sigma}\right)$ if and only if there is a nonzero monomial $M(Q) \in \mathbb{Z}[Q]$ such that $M(Q) P(Q) \in$ $I\left(\mathbb{Q}\left(X_{N}^{(\kappa)}\right)\right)$.

Corollary A.8. The ring $\mathcal{Q}^{\circ}\left(X_{N}^{(\kappa)}\right)$ is isomorphic to $\operatorname{Rep} U_{q}\left(\mathfrak{g}^{\sigma}\right)$ by the correspondence $Q_{m}^{(a)} \mapsto \dot{W}_{m}^{(a)}$.

By taking $q \rightarrow 1$, one can also obtain analogous results for $\operatorname{Rep} \mathfrak{g}^{\sigma}$. 


\section{Appendix B. Proof of (10.9) in Example 10.2(2)}

First we show that $T_{\ell+2}^{(1)}(u), \ldots, T_{\ell+r}^{(1)}(u)$ are in $\operatorname{Ker} \pi_{\ell}$. Let $S_{m}^{(a)}(u):=$ $\pi_{\ell}\left(T_{m}^{(a)}(u)\right)$. Then, we have

$$
\begin{gathered}
\sum_{a=0}^{r+1}(-1)^{a} T_{1}^{(a)}(u+a) T_{m-a}^{(1)}(u+m+a)=\delta_{m, 0}, \\
\sum_{j=0}^{m}(-1)^{j} S_{\ell-1}^{(m-j)}(u+j) S_{\ell-j}^{(1)}(u+j-m)=\delta_{m, 0}, \\
\sum_{a=0}^{k-1}(-1)^{a} S_{1}^{(a)}(u+a) S_{\ell+k-a}^{(1)}(u+\ell+k+a)=0 \quad(1 \leq k \leq r),
\end{gathered}
$$

where $T_{m}^{(0)}(u)=T_{m}^{(r+1)}(u)=S_{m}^{(0)}(u)=S_{m}^{(r+1)}(u)=1(m \geq 0)$ and $T_{m}^{(a)}(u)=$ $S_{m}^{(a)}(u)=0(m<0)$. We obtain (B.1) from the Jacobi-Trudi-type determinant formula in [KNS1, (2.21)], (B.2) from a similar determinant formula in $\mathcal{T}_{\ell}^{\circ}\left(A_{r}\right)$, and (B.3) from (B.1), (B.2), and the half-periodicity $S_{1}^{(a)}(u+\ell+$ $r+1)=S_{\ell-1}^{(r+1-a)}(u)$ in $\mathcal{T}_{\ell}^{\circ}\left(A_{r}\right)$. It follows from (B.3) that $S_{\ell+2}^{(1)}(u)=\cdots=$ $S_{\ell+r}^{(1)}(u)=0$.

Next we prove the following statement in Example 10.2(2).

Proposition B.1. The following relations $\bmod I_{\ell}^{\prime}$ hold in $\mathbb{Z}\left[Y_{a, q^{u}}^{ \pm 1}\right]_{a \in I, u \in \mathbb{C}_{\hbar}}$ :

(1) $Y_{a, q^{u}} Y_{a, q^{u+2}} \cdots Y_{a, q^{u+2 \ell+2 r}} \equiv(-1)^{a r}$,

(2) $Y_{a, q^{u}} \equiv Y_{a, q^{u+2(r+1+\ell)}}$.

Let

$$
a_{u}=\frac{Y_{a, q^{u+a-1}}}{Y_{a-1, q^{u+a}}} \quad(1 \leq a \leq r+1),
$$

where $Y_{0, q^{u}}=Y_{r+1, q^{u}}=1$. We introduce the notation

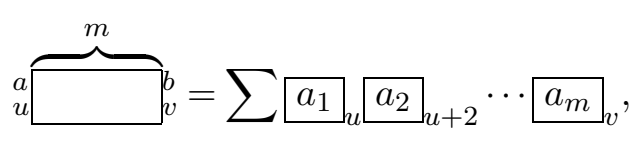

where $v=u+2 m-2$, and the sum extends over all the integers $a_{1}, \ldots, a_{m}$ such that $a \leq a_{1} \leq \cdots \leq a_{m} \leq b$. The array of boxes in the right-hand side is to be understood as the product of the monomials (B.4). By the definition, 
we have the following identities:
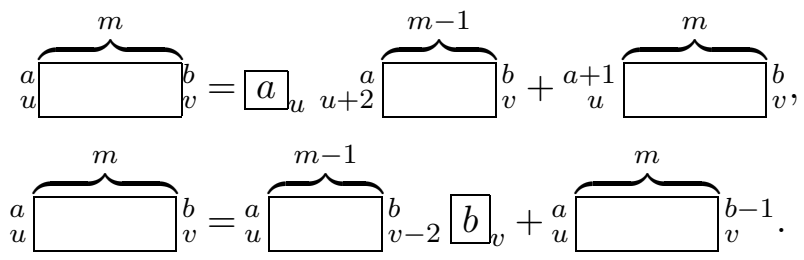

It is well known that the $q$-character of $W_{m}^{(1)}(u)$ is given by

$$
\chi_{q}\left(W_{m}^{(1)}(u)\right)={ }_{u-m+1} \overbrace{\overbrace{}^{m} \begin{array}{l}
r+1 \\
u+m-1
\end{array} .}^{m}
$$

LemmA B.2. Let $1 \leq s \leq r$ and $1 \leq a \leq b \leq r+1$. Then, the following relation $\bmod I_{\ell}^{\prime}$ holds in $\mathbb{Z}\left[Y_{a, q^{u}}^{ \pm 1}\right]_{a \in I, u \in \mathbb{C}_{\hbar}}$ :

$$
\begin{aligned}
& a \overbrace{\overbrace{}^{\ell+s} b}^{b} \\
& \equiv \begin{cases}(-1)^{s} u a-1, \ldots, 2,1 & r+1, r, \ldots, b+1 \\
0 & \text { if } s=r-b+a, \\
\text { if } s>r-b+a .\end{cases}
\end{aligned}
$$

Here $v=u+2(\ell+s-1)$ and the right-hand side of the first case stands for

$$
(-1)^{s} \prod_{\alpha=1}^{a-1} \alpha_{u+2 a-2-2 \alpha} \prod_{\beta=b+1}^{r+1} \beta_{v+2 b+2-2 \beta} .
$$

Proof. We employ the induction on $s$. Suppose $s=1$. Then $s>r-b+a$ happens only if $(a, b)=(1, r+1)$; therefore, $(\mathrm{B} .9)$ is just $\chi_{q}\left(W_{\ell+1}^{(1)}\left(u^{\prime}\right)\right) \equiv 0$ for some $u^{\prime}$. On the other hand, $1=s=r-b+a$ is satisfied for $(a, b)=(2, r+1)$ and $(1, r)$. Thus we show that

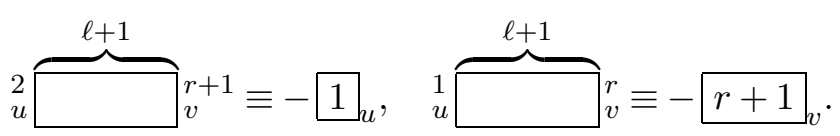

These relations follow from (B.6) and (B.7) by setting $(a, b)=(1, r+1), m=$ $\ell+1$ and using $\chi_{q}\left(W_{\ell}^{(1)}(u)\right) \equiv 1$ and $\chi_{q}\left(W_{\ell+1}^{(1)}(u)\right) \equiv 0$.

Now suppose that (B.9) is valid up to $s-1$. First we consider the case $b-a>r-s$ in (B.9). Setting $u^{\prime}=u+\ell+s-1$ and using (B.6) repeatedly, 
we have

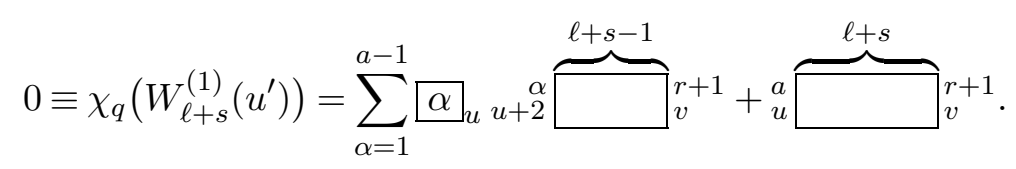

Each term in the $\alpha$ sum is zero $\bmod I_{\ell}^{\prime}$ due to the induction assumption. Applying (B.7) similarly to the resulting relation, we find

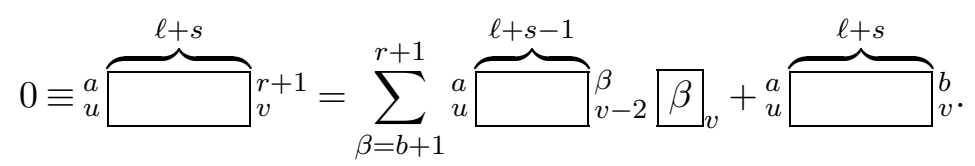

Again, each summand in the $\beta$ sum vanishes $\bmod I_{\ell}^{\prime}$ by the induction assumption, proving the latter case of (B.9).

Next we treat the former case of (B.9); namely, assume that $b-a=r-s$. If $b \leq r$, the same argument (B.10)-(B.11) as above goes through except that the $\beta=b+1$ term in (B.11) is nonvanishing, leading to

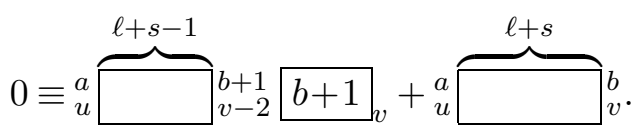

Applying the induction assumption to the first term, we obtain the sought expression for the second term. If $b=r+1$, then $a=b-r+s=s+1$ and the $\alpha$ sum in (B.10) contains nonzero summand at $\alpha=a-1$, leading to

$$
0 \equiv a_{a-1} \begin{gathered}
a-1 \\
u+2
\end{gathered} \overbrace{\varlimsup_{v}^{\ell+s-1}}^{b}+\overbrace{u}^{a} \overbrace{\overbrace{v}^{\ell+s}}^{b} .
$$

Again, rewriting the first term by using the induction assumption yields the sought expression for the second term.

Proof of Proposition B.1. Item (2) is a corollary of (1). To show (1), set $a=b$ and $s=r$ in (B.9). Substituting (B.4) into the resulting relation, we find

$$
\frac{Y_{a, q^{u+1}} Y_{a, q^{u+3}} \cdots Y_{a, q^{u+2 \ell+2 r+1}}}{Y_{a-1, q^{u}} Y_{a-1, q^{u+2}} \cdots Y_{a-1, q^{u+2 \ell+2 r}}} \equiv(-1)^{r} \quad(1 \leq a \leq r+1)
$$

for any $u \in \mathbb{Z}$. From $Y_{0, q^{u}}=1$, the assertion follows. 


\section{REFERENCES}

[A] C. Amiot, Cluster categories for algebras of global dimension 2 and quivers with potential, arXiv:0805.1035.

[ABF] G. E. Andrews, R. J. Baxter, and P. J. Forrester, Eight-vertex SOS model and generalized Rogers-Ramanujan-type identities, J. Stat. Phys., 35 (1984), 193-266.

[ASS] I. Assem, D. Simson, and A. Skowronski, Elements of the Representation Theory of Associative Algebras, Vol. 1, Techniques of Representation Theory, London Math. Soc. Stud. Texts 65, Cambridge University Press, Cambridge, 2006.

[ARS] M. Auslander, I. Reiten, and S. O. Smalo, Representation Theory of Artin Algebras, Cambridge Stud. Adv. Math. 36, Cambridge University Press, Cambridge, 1995.

[BR] V. V. Bazhanov and N. Reshetikhin, Restricted solid-on-solid models connected with simply laced algebras and conformal field theory, J. Phys. A, 23 (1990), 1477-1492.

[B] N. Bourbaki, Groupes et algèbres de Lie, Ch. 4-6, Hermann, Paris, 1968; Masson, Paris, 1981.

[BIRS] A. Buan, O. Iyama, I. Reiten, and J. Scott, Cluster structures for 2-CalabiYau categories and unipotent groups, to appear in Compos. Math., preprint, arXiv:math/0701557.

[BMR] A. Buan, R. Marsh, and I. Reiten, Cluster mutation via quiver representations, Comment. Math. Helv., 83 (2008), 143-177.

[BMRRT] A. B. Buan, R. J. Marsh, M. Reineke, I. Reiten, and G. Todorov, Tilting theory and cluster combinatorics, Adv. in Math., 204 (2006), 572-618.

[CC] P. Caldero and F. Chapoton, Cluster algebras as Hall algebras of quiver representations, Comment. Math. Helv., 81 (2006), 595-616.

[CGT] R. Caracciolo, F. Gliozzi, and R. Tateo, A topological invariant of RG flows in 2D integrable quantum field theories, Int. J. Mod. Phys., 13 (1999), 2927-2932.

[CK1] P. Caldero and B. Keller, From triangulated categories to cluster algebras, Invent. Math., 172 (2008), 169-211.

[CK2] P. Caldero and B. Keller, From triangulated categories to cluster algebras, II, Ann. Sci. École Norm. Sup., 39 (2006), 983-1009.

[CP1] V. Chari and A. Pressley, Quantum affine algebras, Comm. Math. Phys., 142 (1991), 261-283.

[CP2] V. Chari and A. Pressley, Quantum affine algebras and their representations, in Proceedings of Representations of Groups, Banff, 1994, 59-78, CMS Conf. Proc. 16, 1995.

[DeK] R. Dehy and B. Keller, On the combinatorics of rigid objects in 2-Calabi-Yau categories, Int. Math. Res. Not. IMRN, 2008 (2008) rnn029, 17 pages.

[DiK] P. Di Francesco and R. Kedem, Q-systems as cluster algebras II: Cartan matrix of finite type and the polynomial property, arXiv:0803.0362.

[D1] V. Drinfel'd, Hopf algebras and the quantum Yang-Baxter equation, Soviet. Math. Dokl., 32 (1985), 254-258.

[D2] V. Drinfel'd, A new realization of Yangians and quantized affine algebras, Soviet Math. Dokl., 36 (1988), 212-216.

[FZ1] S. Fomin and A. Zelevinsky, Cluster algebras I, Foundations, J. Amer. Math. Soc., 15 (2002), 497-529. 
[FZ2] S. Fomin and A. Zelevinsky, Cluster algebras II, Finite type classification, Invent. Math., 154 (2003), 63-121.

[FZ3] S. Fomin and A. Zelevinsky, Y-systems and generalized associahedra, Ann. of Math., 158 (2003), 977-1018.

[FZ4] S. Fomin and A. Zelevinsky, Cluster algebras IV, Coefficients, Compos. Math., 143 (2007), 112-164.

[FM] E. Frenkel and E. Mukhin, Combinatorics of q-characters of finite-dimensional representations of quantum affine algebras, Comm. Math. Phys., 216 (2001), 23-57.

[FM2] E. Frenkel and E. Mukhin, The q-characters at roots of unity, Adv. Math., 171 (2002), 139-167.

[FR] E. Frenkel and N. Reshetikhin, The q-characters of representations of quantum affine algebras and deformations of W-algebras, Contemp. Math., 248 (1999), 163-205.

[FS] E. Frenkel and A. Szenes, Thermodynamic Bethe ansatz and dilogarithm identities, I, Math. Res. Lett., 2 (1995), 677-693.

[G] P. Gabriel, Auslander-Reiten Sequences and Representation-Finite Algebras, Representation theory, I (Proc. Workshop, Carleton Univ., Ottawa, Ont., 1979), pp. 1-71, Lecture Notes in Math. 831, Springer, Berlin, 1980.

[GT] F. Gliozzi and R. Tateo, Thermodynamic Bethe ansatz and three-fold triangulations, Int. J. Mod. Phys. A, 11 (1996), 4051-4064.

[Ha] D. Happel, Triangulated Categories in the Representation Theory of FiniteDimensional Algebras, London Math. Soc. Lecture Note Ser. 119, Cambridge University Press, Cambridge, 1988.

[HKOTT] G. Hatayama, A. Kuniba, M. Okado, T. Takagi, and Z. Tsuboi, Paths, crystals and fermionic formulae, Math-Phys odyssey 2001, Progr. Math. Phys., 23 (2002), 205-272.

[HKOTY] G. Hatayama, A. Kuniba, M. Okado, T. Takagi, and Y. Yamada, Remarks on fermionic formula, Contemp. Math., 248 (1999), 243-291.

[Hen] A. Henriques, A periodicity theorem for the octahedron recurrence, J. Algebraic Combin., 26 (2007), 1-26.

[Her1] D. Hernandez, The Kirillov-Reshetikhin conjecture and solutions of T-systems, J. Reine Angew. Math., 596 (2006), 63-87.

[Her2] D. Hernandez, The Kirillov-Reshetikhin conjecture: The general case, arXiv:0704.2838.

[HL] D. Hernandez and B. Leclerc, Cluster algebras and quantum affine algebras, in preparation; and talk presented by B. Leclerc at Workshop "Lie Theory" held at MSRI, Berkeley, March 2008.

[Hi1] R. Hirota, Nonlinear partial difference equations II: Discrete time Toda equations, J. Phys. Soc. Japan, 43 (1977), 2074-2078.

[Hi2] R. Hirota, Discrete two-dimensional Toda molecule equation, J. Phys. Soc. Japan, 56 (1987), 4285-4288.

[IY] O. Iyama and Y. Yoshino, Mutation in triangulated categories and rigid CohenMacaulay modules, Invent. Math., 172 (2008), 117-168.

[J] M. Jimbo, A q-difference analogue of $U(\hat{\mathfrak{g}})$ and the Yang-Baxter equation, Lett. Math. Phys., 10 (1985), 63-69.

[JMO] M. Jimbo, T. Miwa, and M. Okado, Solvable lattice models related to the vector representation of classical simple Lie algebras, Comm. Math. Phys., 116 (1988), 507-525. 
[Ka] V. G. Kac, Infinite Dimensional Lie Algebras, 3rd ed., Cambridge University Press, 1990.

[Ked] R. Kedem, Q-systems as cluster algebras, arXiv:0712.2695.

[Kel1] B. Keller, On triangulated orbit categories, Doc. Math., 10 (2005), 551-581.

[Kel2] B. Keller, Cluster algebras, quiver representations and triangulated categories, arXiv:0807.1960.

[Kel3] B. Keller, The periodicity conjecture for pairs of Dynkin diagrams, in preparation.

[Kel4] B. Keller, Deformed CY-completions and their duals, in preparation.

[Ki] A. N. Kirillov, Identities for the Rogers dilogarithm function connected with simple Lie algebras, J. Sov. Math., 47 (1989), 2450-2459.

[Ki2] A. N. Kirillov, private communication.

[KR] A. N. Kirillov and N. Reshetikhin, Representations of Yangians and multiplicities of the inclusion of the irreducible components of the tensor product of representations of simple Lie algebras, J. Sov. Math., 52 (1990), 3156-3164.

[KP] A. Klümper and P. A. Pearce, Conformal weights of RSOS lattice models and their fusion hierarchies, Phys. A, 183 (1992), 304-350.

[Kn] H. Knight, Spectra of tensor products of finite-dimensional representations of Yangians, J. Algebra, 174 (1995), 187-196.

[KLWZ] I. Krichever, O. Lipan, P. Wiegmann, and A. Zabrodin, Quantum integrable models and discrete classical Hirota equations, Comm. Math. Phys., 188 (1997), 267-304.

[Ku] A. Kuniba, Thermodynamics of the $U_{q}\left(X_{r}^{(1)}\right)$ Bethe ansatz system with $q$ a root of unity, Nucl. Phys. B, 389 (1993), 209-244.

[KN] A. Kuniba and T. Nakanishi, Spectra in conformal field theories from the Rogers dilogarithm, Mod. Phys. Lett. A, 7 (1992), 3487-3494.

[KNS1] A. Kuniba, T. Nakanishi, and J. Suzuki, Functional relations in solvable lattice models: I. Functional relations and representation theory, Int. J. Mod. Phys. A, 9 (1994), 5215-5266.

[KNS2] A. Kuniba, T. Nakanishi, and J. Suzuki, Functional relations in solvable lattice models: II. Applications, Int. J. Mod. Phys. A, 9 (1994), 5267-5312.

[KNT] A. Kuniba, T. Nakanishi, and Z. Tsuboi, The canonical solutions of the Qsystems and the Kirillov-Reshetikhin conjecture, Comm. Math. Phys., 227 (2002), 155-190.

[KOS] A. Kuniba, Y. Ohta, and J. Suzuki, Quantum Jacobi-Trudi and Giambelli formulae for $U_{q}\left(B_{r}^{(1)}\right)$ from the analytic Bethe ansatz, J. Phys. A, 28 (1995), 6211-6226.

[KOSY] A. Kuniba, M. Okado, J. Suzuki, and Y. Yamada, Difference L operators related to q-characters, J. Phys. A, 35 (2002), 1415-1435.

[KS] A. Kuniba and J. Suzuki, Functional relations and analytic Bethe ansatz for twisted quantum affine algebras, J. Phys. A, 28 (1995), 711-722.

[N1] H. Nakajima, Quiver varieties and finite dimensional representations of quantum affine algebras, J. Amer. Math. Soc., 14 (2001), 145-238.

[N2] H. Nakajima, Quiver varieties and t-analogues of q-characters of quantum affine algebras, Ann. of Math., 160 (2004), 1057-1097.

[N3] H. Nakajima, t-Analogs of q-characters of Kirillov-Reshetikhin modules of quantum affine algebras, Represent. Theory, 7 (2003), 259-274. 
[Pal] Y. Palu, Cluster characters for triangulated 2-Calabi-Yau categories, Ann. Inst. Fourier (Grenoble), 58 (2008), 2221-2248.

[Pas] V. Pasquier, Etiology of IRF models, Comm. Math. Phys., 118 (1988), 335364.

[RTV] F. Ravanini, R. Tateo, and A. Valleriani, Dynkin TBA's, Int. J. Mod. Phys. A, 8 (1993), 1707-1727.

[S] M. Sato, Soliton equations as dynamical systems on infinite dimensional Grassmann manifolds, RIMS Kôkyûroku Bessatsu, 439 (1981), 30-46.

[V] A. Y. Volkov, On the periodicity conjecture for $Y$-systems, Comm. Math. Phys., 276 (2007), 509-517.

[YZ] S.-W. Yang and A. Zelevinsky, Cluster algebras of finite type via Coxeter elements and principal minors, arXiv:0804.3303.

[Z] Al. B. Zamolodchikov, On the thermodynamic Bethe ansatz equations for reflectionless ADE scattering theories, Phys. Lett. B, 253 (1991), 391-394.

Rei Inoue

Faculty of Pharmaceutical Sciences

Suzuka University of Medical Science

Suzuka, 513-8670

Japan

reiiy@suzuka-u.ac.jp

Osamu Iyama

Graduate School of Mathematics

Nagoya University

Nagoya, 464-8604

Japan

iyama@math.nagoya-u.ac.jp

Atsuo Kuniba

Institute of Physics

University of Tokyo

Tokyo, 153-8902

Japan

atsuo@gokutan.c.c-tokyo.ac.jp

Tomoki Nakanishi

Graduate School of Mathematics

Nagoya University

Nagoya, 464-8604

Japan

nakanisi@math.nagoya-u.ac.jp 
Junji Suzuki

Department of Physics

Faculty of Science

Shizuoka University

Ohya, 836

Japan

sjsuzuk@ipc.shizuko.ka.ac.jp 